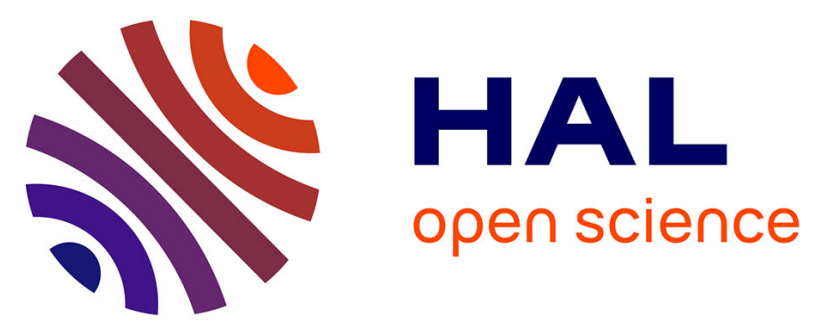

\title{
Pre-Variscan tectonic setting of the south margin of Armorica: Insights from detrital zircon ages distribution and Hf isotopic composition of the St-Georges-sur-Loire Unit (S. Armorican Massif, France)
}

Wei Lin, Michel Faure, Xian-Hua Li, Wenbin Ji

\section{To cite this version:}

Wei Lin, Michel Faure, Xian-Hua Li, Wenbin Ji. Pre-Variscan tectonic setting of the south margin of Armorica: Insights from detrital zircon ages distribution and Hf isotopic composition of the StGeorges-sur-Loire Unit (S. Armorican Massif, France). Tectonophysics, 2019, 766 (5), pp.340-378. 10.1016/j.tecto.2019.06.015 . insu-02174597

\author{
HAL Id: insu-02174597 \\ https://hal-insu.archives-ouvertes.fr/insu-02174597
}

Submitted on 5 Jul 2019

HAL is a multi-disciplinary open access archive for the deposit and dissemination of scientific research documents, whether they are published or not. The documents may come from teaching and research institutions in France or abroad, or from public or private research centers.
L'archive ouverte pluridisciplinaire HAL, est destinée au dépôt et à la diffusion de documents scientifiques de niveau recherche, publiés ou non, émanant des établissements d'enseignement et de recherche français ou étrangers, des laboratoires publics ou privés.

\section{(ㅇ)(1) $\$$}

Distributed under a Creative Commons Attribution - NonCommercial - NoDerivatives 44.0 


\section{Accepted Manuscript}

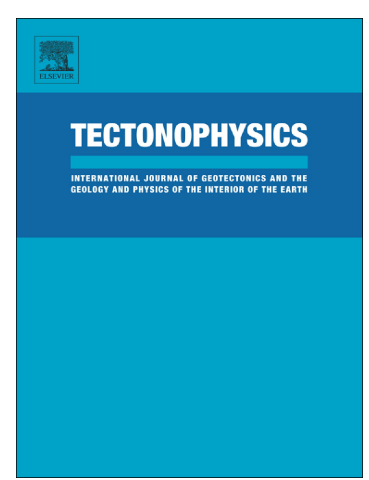

Wei Lin, Michel Faure, Xian-Hua Li, Wenbin Ji

PII: $\quad$ S0040-1951(19)30246-X

DOI: $\quad$ https://doi.org/10.1016/j.tecto.2019.06.015

Reference: $\quad$ TECTO 128145

To appear in: Tectonophysics

Received date: $\quad 26$ January 2019

Revised date: $\quad 15$ June 2019

Accepted date: $\quad 21$ June 2019

Please cite this article as: W. Lin, M. Faure, X.-H. Li, et al., Pre-Variscan tectonic setting of the south margin of Armorica: Insights from detrital zircon ages distribution and $\mathrm{Hf}$ isotopic composition of the St-Georges-sur-Loire Unit (S. Armorican Massif, France), Tectonophysics, https://doi.org/10.1016/j.tecto.2019.06.015

This is a PDF file of an unedited manuscript that has been accepted for publication. As a service to our customers we are providing this early version of the manuscript. The manuscript will undergo copyediting, typesetting, and review of the resulting proof before it is published in its final form. Please note that during the production process errors may be discovered which could affect the content, and all legal disclaimers that apply to the journal pertain. 
Pre-Variscan tectonic setting of the south margin of Armorica: Insights from detrital zircon ages distribution and Hf isotopic composition of the $\mathrm{S}^{\mathrm{t}}$-Georgessur-Loire Unit (S. Armorican Massif, France)

Wei Lin ${ }^{\mathrm{a}, \mathrm{c}}$, Michel Faure ${ }^{\mathrm{b}}$, Xian-Hua Li ${ }^{\mathrm{a}, \mathrm{c}}$, Wenbin $\mathrm{Ji}^{\mathrm{d}}$

${ }^{a}$ State Key Laboratory of Lithospheric Evolution, Institute of Geology and Geophysics, Institutions of Earth Science, Chinese Academy of Sciences, Beijing 100029, China

${ }^{\mathrm{b}}$ ISTO, Université d'Orléans, UMR 7327, 45071 Orléans Cedex 2, France

${ }^{\mathrm{c}}$ College of Earth and Planetary Sciences, University of Chinese Academy of Sciences, Beijing 100049, China

${ }^{\mathrm{d}}$ State Key Laboratory of Continental Dynamics, Department of Geology, Northwest University, No. 229 Taibai Northern Road, Xi'an 710069, China

Corresponding author. Tel.: +86 10 82998513; fax: +861062010846.

E-mail address: linwei@ mail.iggcas.ac.cn (W. Lin).

\section{Abstract}

The Armorican Massif experienced a poly-orogenic evolution, represented by the Neoproterozoic Cadomian and the Devonian-Carboniferous Variscan tectonothermal events. In the southernmost part of the Armorican Domain (or Armorica microcontinent), the $\mathrm{S}^{\mathrm{t}}$ Georges-sur-Loire Unit consists of a block-in-matrix subunit, and a turbiditic subunit in the South and North, respectively. The $\mathrm{S}^{\mathrm{t}}$-Georges-sur Loire unit is interpreted as a remnant backarc basin formed in Silurian-Early Devonian (ca. 440-400 Ma), and was deformed during the 
Variscan orogeny. Most of the sedimentary and magmatic blocks enclosed in clastic matrix are olistoliths derived from an assumed southern area called the "missing domain", presently exposed as the Variscan Mauges Nappe composed of the Cadomian rocks and Early Paleozoic sedimentary cover. The "missing domain" was subducted beneath the Armorican massif during the Variscan collision. Detrital zircon age spectra from sandstones and siltstones from the olistostrome matrix reveal Neoproterozoic and Early Paleozoic age clusters, and less abundant Paleoproterozoic and Archean ages. Hf isotopic compositions of detrital zircons indicate dominantly negative $\varepsilon_{\mathrm{Hf}}(\mathrm{t})$ values which are in agreement with a recycled continental source. Minor positive values correspond to a juvenile component that was formed during the Neoproterozoic Cadomian orogeny or Archean-Paleoproterozoic events. The Cambrian and Early Ordovician ages can be related to the pre-Variscan rifting of the Pannotia megacontinent that separated the Armorica microcontinent from Gondwana. Neoproterozoic ages imply a source of the magmatic rocks exposed in the Cadomian belt of the northern part of the Armorica microcontinent, and in the Mauges Nappe.

Key words: U-Pb-Hf of detrital zircons, Provenance, Cadomian belt, Variscan belt, Armorica microcontinent

\section{Introduction}

Detrital zircon U-Pb dating is a powerful tool to assess the provenance of clastic formations (e.g. Fedo et al., 2003; Cawood et al., 2007, 2012; Dickinson and Gerhels, 2009). Combined with in-situ Hf-isotope analysis, this approach can also provide useful information for reconstruction of the tectonic evolution of continental blocks, and discrimination of crust or mantle contributions (Griffin et al., 2000; Condie et al., 2009). This method has been widely applied in various orogenic belts, for instance the Variscan-Appalachian orogen 
(Henderson et al., 2016), the Himalaya, and the Alps (Gehrels et al., 2011; Cai et al., 2016; Chu et al., 2016; Lin et al., 2018). The European Variscan belt is well investigated in Iberia (e.g. Martínez Catalán et al., 2008, 2009), the Bohemian massif (e.g. Henderson et al., 2016; Linneman et al., 2004, 2007), and Corsica (Avigad et al., 2018). In the French Variscan orogen, a few studies deal with detrital zircon age distributions of the French Massif Central, namely in its western part, or Limousin (Melleton et al., 2010), and its southern part, in Montagne Noire, where the turbiditic foreland basin is well exposed (Lin et al., 2016). In the Armorican Massif, investigations on detrital zircon age distribution have only been carried out in the Central Armorican Domain (Ballouard et al., 2018), the Early Carboniferous Ancenis Basin, and the Late Carboniferous "coal trough" (Ducassou et al., 2014). This paper aims to provide new data on the age distribution and Hf isotopic composition of the detrital zircons in the matrix of the olistostrome of the $S^{t}$-Georges sur Loire unit on the southern margin of the Armorica microcontinent, in order to discuss the provenance of the rocks in the unit, and to clarify the tectonic relationships between the different units of the Ligerian Domain during Variscan subduction. The sources of the detrital zircons and their Hf isotopic signatures with respect to the Neoproterozoic Cadomian orogen, and the basement of the Armorican Massif, are also discussed.

\section{Geological outline of the Armorican Massif}

The French Armorican Massif experienced poly-orogenic superimposition, namely, the Late Neoproterozoic Cadomian orogeny, and the Late Paleozoic Variscan orogeny (Chantraine et al., 2001; Ballèvre et al., 2009, and references therein). The Armorican Massif is the only place in France that records the structural relationships between the Armorica microcontinent to the north, and the North Gondwana Margin to the south. In the Armorican massif, three domains are separated by ophiolitic sutures, and they correspond to different 
microcontinents with Gondwana affinities (e.g. Faure et al., 2005, 2008; Ballèvre et al., 2009; Fig. 1). From north to south, these domains are: i) the Léon Domain formed by N-directed metamorphic nappes (Rolet et al., 1994; Faure et al., 2010); ii) the North-and-Central Armorican Domain that consists of deformed and metamorphosed Neoproterozoic rocks related to the ca. 650-540 Ma Cadomian orogeny, and weakly deformed Paleozoic sedimentary cover (e.g. Chantraine et al., 2001); iii) the South Armorican Domain that includes a stack of S-directed metamorphic nappes. In terms of Variscan geodynamics, the Léon, North-and-Central Armorican, and South Armorican domains belong to the MidGerman Crystalline Rise, Armorica, and North Gondwana margin, respectively. All these domains were rifted from Gondwana during the Early Ordovician and subsequently assembled together during the Variscan orogeny.

The Léon domain is not described here as it is not relevant to our study. The Northand-Central Armorican Domain contains an isolated fragment of the Cadomian belt (Fig. 1). This Neoproterozoic accretionary orogen has been subdivided into several NE-SW trending units that verge to the SE (e. g. Dissler et al., 1988; Chantraine et al., 2001 and references therein). To the NW, the Trégor Unit includes a Paleoproterozoic basement, also known as the Icartian basement, with ca. $2035 \pm 35$ Ma orthogneiss, and amphibolites, and a calcalkaline volcanic-plutonic complex (Chantraine et al., 2001). These magmatic rocks yielding Ediacaran ages (ca $615 \mathrm{Ma}$ ) are interpreted as formed in a magmatic arc. To the SE, $\mathrm{S}^{\mathrm{t}}$-Brieuc Unit is formed by a bimodal volcanic suite dated at ca $588 \pm 11 \mathrm{Ma}$, and a volcanic-clastic series intruded by 590-580 Ma gabbro-dioritic, dioritic or gabbroic plutons. The lower part of the $S^{t}$-Brieuc Unit is underlain by a 750-650 Ma orthogneiss (Egal et al., 1996; Chantraine et al., 2001). The $\mathrm{S}^{\mathrm{t}}$-Brieuc Unit is interpreted as a back-arc basin opened within an EoCadomian arc (Dissler et al., 1988; Chantraine et al., 2001). The Guingamp and St-Malo Units are characterized by high temperature gneiss, and migmatites developed at the expense of 
Cryogenian to Ediacaran metasediments. The $\mathrm{S}^{\mathrm{t}}$-Malo migmatite and the Cancale leucogranite

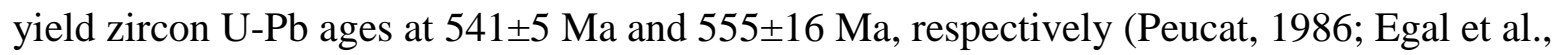
1996). Lastly, the southeasternmost Fougères Unit consists of turbidites intruded by Early Cambrian (ca. 540 Ma, Pasteels and Doré, 1982) peraluminous granites, referred to as the Mancellian batholith (Graviou et al., 1988; Guerrot and Peucat, 1990). The Fougères Unit, devoid of metamorphism and ductile deformation, is interpreted as the Cadomian foreland basin filled by eroded material supplied from the Trégor and $\mathrm{S}^{\mathrm{t}}$-Brieuc units. Below the Early Ordovician unconformity, the Neoproterozoic rocks that crop out in Central Brittany consist of graywackes, sandstone and mudstone with volcanic intercalations. Their precise age and structure are poorly documented. A recent study combining trace fossils and detrital zircon age investigations were indicative of an Ediacaran-Fortunian age (Gougeon et al., 2018). These formations are considered as the southern distal extension of the Fougères Unit.

The Conquet and Nort-sur Erdre faults correspond to the ophiolitic sutures that separate the three domains (Fig. 1). In addition to these major paleogeodynamic boundaries, the Armorican Massif is cut by West-East to NW-SE striking dextral strike-slip faults of the North Armorican Shear Zone, North branch of the South Armorican Shear Zone, and the South Branch of the South Armorican Shear Zone (Jégouzo, 1980).

In the SE part of the Armorican Massif, on the two sides of the Nort-sur Erdre fault, the Variscan deformation both affected the southern margin of Armorica, and the northernmost part of the North Gondwana margin. The triangular area located between the north and south branches the South Armorican Shear Zone is also referred to as the Ligerian Domain (Cavet et al., 1970, 1978; Lardeux and Cavet, 1994; Cartier et al., 2001; Cartier and Faure, 2004; Ducassou et al., 2009, 2011, 2014; Figs. 2 and 3). Several lithotectonic units form the Ligerian domain (Figs. 2 and 3). Within this domain, the Lanvaux Unit consists of the Neoproterozoic to the Early Ordovician sedimentary and volcanic rocks intruded by the 
Early Ordovician alkaline granites (Vidal, 1980; Ballèvre et al., 2009), now deformed as orthogneiss, exposed near Redon and Angers. The $S^{t}$-Georges-sur-Loire Unit is a clastic unit subdivided into a northern turbiditic sub-unit, and a southern olistostrome sub-unit (cf. details below). The Nort-sur-Erdre fault is the southern boundary of the $S^{t}$-Georges-sur-Loire Unit and represents the Eo-Variscan suture (Faure et al., 2005, 2008). Thus, together with the Lanvaux Unit, the $\mathrm{S}^{\mathrm{t}}$-Georges-sur-Loire Unit belongs to the North and Central Armorican Domain (i.e. Armorica). The age of the suture is still disputed; some authors argue for $360 \mathrm{Ma}$ (e.g. Ballèvre et al., 2008, Paquette et al., 2017), whereas others propose a ca 400 Ma age (Pin, 1990; Faure et al., 2005, 2009).

South of the Nort-sur-Erdre fault (i.e. the Eo-Variscan suture), several litho-tectonic units occur. Adjacent to the fault, the Late Carboniferous sandstone with coal measures forms the "Sillon Houiller de la Basse-Loire" (Lower Loire coal trough). Early Carboniferous (Tournaisian-Visean) clastic rocks filled the Ancenis Basin. As indicated by the kinematics of the boundary fault and drag folds, the spindle-shaped Ancenis Basin opened as a left-lateral pull apart superimposed upon the Mauges Nappe (Diot and Blaise, 1978). Neoproterozoic metapelite, greywacke, and basalt are the main constituents of the Mauges Nappe. The metamorphic volcanic-sedimentary series is uncomformably overlain by Cambrian conglomerate, sandstone and rhyolitic lava flows, and Ordovician sandstone. The Mauges Nappe overthrusts to the S (SW) an ophiolitic nappe composed of an assemblage of amphibolite, metagabbro, serpentinite (referred to as the Drain Unit), and chloritoid-chloritegarnet metapelite called the Hâvre unit (Marchand, 1981; Ballèvre et al., 2009). A mylonitic metagabbro yields a zircon $\mathrm{U}-\mathrm{Pb}$ age at $381.8 \pm 2.3 \mathrm{Ma}$, interpreted as the magmatic crystallization age of this gabbro (Paquette et al., 2017). This nappe overthrusts the Champtoceaux complex formed by imbricated thrust sheets of high pressure rocks with mafic eclogites, orthogneiss, and paragneiss and locally migmatite. The mafic eclogites are 
commonly retrogressed to garnet amphibolite enclosed in variously melted metapelites and orthogneisses. Monazite from a migmatitic leucosome yields a U-Th-Pb chemical age of $387 \pm 6 \mathrm{Ma}$ (Cocherie et al., 2005). The lowermost part of the stack of nappes comprises the Mauves Unit, which consists of paragneiss and micaschists that did not experience the HP metamorphism characteristic of the Champtoceaux complex (Marchand, 1981; Ballèvre et al., 2009). The nappe pile was folded to form an E-W trending upright antiform, the core of which is occupied by late Carboniferous two mica plutons. Most of these leucogranites develop S-C structures coeval with the dextral shearing of the southern branch of the South Armorican Shear Zone (Berthé et al., 1978; Jégouzo, 1980).

\section{The $S^{t}$-Georges-sur-Loire Unit}

North of the Nort-sur-Erdre fault, the southern part of the $\mathrm{S}^{\mathrm{t}}$-Georges-sur-Loire Unit is interpreted as a "block-in-matrix" formation, or olistostrome (Dubreuil, 1986; Cartier et al., 2001; Cartier and Faure, 2004; Fig. 4A, B). The main blocks consist of Silurian black chert (Fig. 4G), Late Silurian to Middle Devonian limestone (Fig. 4D), sandstone (Fig. 4C), spilitized mafic lava (Fig. 4H) with porphyritic basalt, pillow basalt, dolerite, gabbro, rhyolite, microgranite, andesite and trachyte (see details in Cartier and Faure, 2004). Geochemical analyses suggest that the mafic rocks were formed either in an active continental margin or in a back-arc basin partly floored by oceanic crust (Carpentier et al., 1982; Cabanis, 1986; Cartier and Faure, 2004). The matrix of the olistostrome commonly consists of pelite, silt or sandstone, and is described as a flysch deposit (Lardeux and Cavet, 1994; Fig. 4A, B, E, and F). Pebbly mudstones, with mm- to cm-sized clasts, are also representative of a gravitydriven, chaotic sedimentation deposited in a tectonically active region. Both the olistostrome and the sandstone-pelite sub-units experienced three phases of ductile deformation (Cartier and Faure, 2004). 
The source of the olistoliths is controversial. A northern origin from the North-andCentral Armorican Domain is ruled out by the lithological and paleontological differences of the Paleozoic successions between the two domains (Dubreuil, 1986; Lardeux and Cavet, 1994). However, the formations exposed to the South of the Nort-sur-Erdre fault are only represented by metamorphic rocks, except for the Cambrian-Ordovician sedimentary cover of the Mauges Nappe. In particular, the Cambrian rhyolite that overlies the Neoproterozoic micaschist might have supplied blocks to the $\mathrm{S}^{\mathrm{t}}$-Georges-sur-Loire Unit. The southern boundary of the Carboniferous Ancenis Basin contains Ordovician to Emsian clastic formations (Ducassou et al., 2009, 2011), but magmatic rocks are absent there. Therefore, following a previous interpretation (Dubreuil, 1986), the possibility of a "missing domain" located between the $\mathrm{S}^{\mathrm{t}}$-Georges-sur-Loire Unit and the Mauges Nappe has been proposed (Faure et al., 2008). In this interpretation, a magmatic arc developed upon a Neoproterozoic Cadomian basement was separated from Armorica by a back-arc basin. During the Variscan tectonics, a part of the back-arc basin, represented by the $\mathrm{S}^{\mathrm{t}}$-Georges-sur-Loire Unit, was folded and thrusted to the N or NW upon the Lanvaux Unit. The South Armorican nappe stack was formed by the Mauges Nappe, the ophiolitic nappe, and the Champtoceaux Complex resulted from the North-directed continental subduction of the North Gondwana margin. The reduced lithosphere thickness, below the back-arc basin, played a role in the breakup of the upper plate. This tectonic regime led to the subduction of the magmatic arc, and a great part of the back-arc basin. Consequently, the present $\mathrm{S}^{\mathrm{t}}$-Georges-sur-Loire Unit, and the Mauges Nappe are the only remnants of this back-arc-arc system developed along the active margin of the southern Armorica microcontinent.

\section{Petrography of the analyzed samples}


Detrital zircon $\mathrm{U}-\mathrm{Pb}$ age spectra and isotopic $\mathrm{Lu}-\mathrm{Hf}$ proved to be an exceedingly useful tool to identify a geological history of sedimentary basins in orogenic belts due to the robustness of zircon to later thermal events and surface processes (Gehrels and Dickinson, 1995; Bruguier et al., 1997; Fedo et al., 2003). Since the proportion of zircons with different ages does not change drastically during sediment transport, owing to the inherent stability of zircon, they can reveal the history of the source area eroded continental crust and sedimentary regime in an ancient arc-trench system. In order to reveal the pre-Variscan lithological history of the south margin of Armorica block, eight samples from the St-Georges-sur-Loire Unit have been analyzed for zircon dating. Their locations are presented in Fig. 2, and the GPS coordinates are provided in Table 1. Seven samples (13FR12, 13FR18-1, 13FR19, 13FR21, OUT, MB2, RO2) are sandstone or siltstone from the olistostrome matrix. Sample 13FR13 is a rhyolitic block enclosed in the sandstone matrix of the olistostrome. The clastic matrix consists dominantly of rounded quartz grains, although recrystallization developed during the ductile deformation led to a smoothed grain boundary with pressure shadows. Other clasts found in the sandstone are plagioclase and muscovite (Fig. 5). In thin section, quartz grain embayments are indicative of a volcanic or hypovolcanic source (Fig. 5B).

\section{Analytical procedures}

\subsection{Zircon $U-P b$ dating}

Zircons were separated from each sample of ca. $2 \mathrm{~kg}$ in weight using standard heavy liquid and magnetic separation techniques. Zircon grains, together with standard 91500 and Temora zircons were cast in an epoxy mount, which were then polished to reveal the grain interiors for analysis. These grains were randomly selected in different sizes to avoid any age bias based on grain size. Zircon structures were documented with transmitted and reflected 
light micrographs, and cathodoluminescence (CL) images were obtained by a CAMECA electron microscope in order to reveal their internal structures.

On the basis of these analyzed photographs, various grains were selected for analysis. Zircon U-Pb dating for seven sandstones (13FR12, 13FR18-1, 13FR19, 13FR21, OUT, MB2, RO2) and one rhyolite (13FR13) sample was analyzed using a quadrupole ICP-MS (Agilent 7500a) equipped with a $193 \mathrm{~nm}$ laser-ablation system (Analyte G2) at the Institute of Geology and Geophysics, Chinese Academy of Sciences in Beijing. U-Th- $\mathrm{Pb}$ ratios and absolute abundances were determined relative to the standard zircon. Detailed analytical procedures are described by Xie et al. (2008). The spot diameter is $40 \mu \mathrm{m}$ in size. Correction of common lead was applied following the method described by Andersen (2002). The GLITTER program was used for data processing (van Achterbergh et al., 2001). Data plotting was carried out using the Density Plotter program (Vermeesch, 2012). LA-ICPMS zircon U-Pb analytical data are presented in Table 2 . Uncertainties on individual analyses in data tables are reported at a $1 \sigma$ level. Analyses that are $>15 \%$ discordant (by comparison of ${ }^{206} \mathrm{~Pb} /{ }^{238} \mathrm{U}$ and ${ }^{207} \mathrm{~Pb}^{206} \mathrm{~Pb}$ ages) are excluded (Table 2). Zircon ages younger than $1000 \mathrm{Ma}$ were based on ${ }^{206} \mathrm{~Pb} /{ }^{238} \mathrm{U}$ ratios, and ages older than $1000 \mathrm{Ma}$ were based on ${ }^{207} \mathrm{~Pb} /{ }^{206} \mathrm{~Pb}$ ratios.

\subsection{Zircon Lu-Hf isotopes}

Zircon Lu-Hf isotopic analysis was carried out in situ on a Neptune multi-collector ICPMS equipped with a Geolas-193 laser ablation system at the Institute of Geology and Geophysics, Chinese Academy of Sciences. Previously analyzed zircon grains for U-Pb isotopes were chosen for Lu-Hf isotopic analyses, with ablation pits of 44 or $60 \mu \mathrm{m}$ in diameter, ablation time of 26 seconds, repetition rate of $10 \mathrm{~Hz}$, and laser beam energy density of $10 \mathrm{~J} / \mathrm{cm}^{2}$. The detailed analytical procedures are similar to those described by $\mathrm{Wu}$ et al. 
(2006). Measured ${ }^{176} \mathrm{Hf} /{ }^{177} \mathrm{Hf}$ ratios were normalized to ${ }^{179} \mathrm{Hf} /{ }^{177} \mathrm{Hf}=0.7325$. Zircon standards Mud Tank and GJ-1 were analyzed alternately with the unknowns. During the course of this study, we obtained ${ }^{176} \mathrm{Hf} /{ }^{177} \mathrm{Hf}$ ratio of $0.282504 \pm 0.000032(2 \sigma, \mathrm{n}=58)$ and $0.282022 \pm 0.000051(2 \sigma, \mathrm{n}=58)$ for Mud Tank and GJ-1, respectively, in good agreement within errors with the recommended values (Woodhead and Hergt, 2005; Morel et al., 2008).

\section{Zircon U-Pb and Lu-Hf analytical results}

Representative CL images of the dated zircons are shown in Fig. 6. All analyzed samples, yield zircon grains with a wide range in size from $50 \mu \mathrm{m}$ to $200 \mu \mathrm{m}$ (Fig. 6). Except for a few prismatic crystals, most of the grains possess a rounded shape with abraded crystallographic faces, indicating long fluvial transportation. Most of the grains exhibit a zonal structure, sometimes with an inner core surrounded by recrystallization rims with clear oscillatory zoning.

Lu-Hf isotopes were obtained on dated zircon grains, with analytical spots adjacent to those for U-Pb dating, or in the same growth domain as inferred from CL imagery (Fig. 6). U$\mathrm{Pb}$ ages and $\varepsilon_{\mathrm{Hf}}(\mathrm{t})$ results of individual samples are plotted in Figs. 7 and 8. Diagrams of $\varepsilon_{\mathrm{Hf}}(\mathrm{t})$ values are presented in Fig. 12, and data are summarized in Table 3. The zircon two-stage model Hf age $\left(\mathrm{T}_{\mathrm{DM}}{ }^{2}\right)$ are presented in Figs. 9 and 10.

\subsection{FR12}

Sample 13FR12, from Champtocé (Figs. 2, 4A, Table 1), is a middle- to fine-grained sandstone. The grain size is around $0.18-0.40 \mathrm{~mm}$ and commonly $0.25-0.35 \mathrm{~mm}$ (Fig. 5A). Major components are quartz, plagioclase, muscovite, and lithic fragments. In thin section, 
poorly rounded quartz with undulatory extinction represents $75-78 \%$ of the detrital component. Plagioclase and muscovite, less than 1\%, are rarely observed (Fig. 5A).

In this sample, a total of 100 points on 100 grains were analyzed, and 94 analyses were concordant (Table 2). Concentric zoning is distinctly demonstrated in euhedral zircon grains with 70 to $250 \mu \mathrm{m}$ in length, rounded or prismatic shape, and length-width ratios of $1: 1$ to 2.5:1. Th/U ratios range from 0.01 to 2.52 . Except one grain, $\mathrm{Th} / \mathrm{U}$ ratios are higher than 0.1 (Table 2). Combined with the CL images, these compositions and morphometric features of the zircons are indicative of a magmatic origin (Fig. 6). The measured apparent ages range from $3300 \mathrm{Ma}$ to $458 \mathrm{Ma}$ (Table 2). Two major age groups occur at 760-450 Ma and 2210$1560 \mathrm{Ma}$, and one subordinate group at 2850-2500 Ma (Fig. 7). Two dominant age peaks are around $1865 \mathrm{Ma}$ and $595 \mathrm{Ma}$, with a subordinate age peak at ca. $2050 \mathrm{Ma}$. A low background of dispersed ages from Mesoarchean to the Neoarchean is also demonstrated (Fig. 7).

Forty dated zircons were chosen for in situ $\mathrm{Hf}$ isotope analyses with ${ }^{176} \mathrm{Hf} /{ }^{177} \mathrm{Hf}$ ratios varying from 0.280867 to 0.282355 (Fig. 6; Table 3). All the analyses have $\varepsilon_{\mathrm{Hf}}(\mathrm{t})$ values of +6.3 to -23.9 with the two dominant peaks at -4.5 and -9.9 , and a subordinate peak at -18.4 (Fig. 8). In sample 13FR12, zircon two-stage model Hf ages $\left(\mathrm{T}_{\mathrm{DM}}{ }^{2}\right)$ range from 1.72 to 3.70 Ga with two clusters around $2225 \mathrm{Ma}$, and $2820 \mathrm{Ma}$ (Fig. 9; Table 3).

\subsection{FR13}

Sample 13FR13 is a rhyolitic block in a siltstone matrix at Epiré, and represents the olistostrome sub-unit of the $\mathrm{S}^{\mathrm{t}}$-Georges-sur-Loire Unit (Fig. 2, Table 1). In thin section, this sample is rich in plagioclase with grain sizes from $0.1 \mathrm{~mm}$ to $\sim 5 \mathrm{~mm}$ (Fig. $5 \mathrm{~B}$ ). The major components are polycrystalline aggregates of quartz, plagioclase, and orthoclase phenocrysts. 
Microcrystalline or aphanitic quartz and plagioclase appear as mesostasis. Opaque minerals are also present. Magmatic minerals are still preserved. No secondary plagioclase was observed in the thin section. Concentric zoning is distinct in euhedral zircon grains with 110 to $300 \mu \mathrm{m}$ in length, prismatic shape, and length-width ratios of $1.5: 1$ to $4: 1$ (Fig. 6). Th/U ratios range from 0.05 to 1.53 . Except for two grains, these zircons have $\mathrm{Th} / \mathrm{U}$ ratios higher than 0.1 (Table 2).

A total of 90 analyses on 90 grains were made, of which 10 analyses are discordant (Table 2). 80 concordant ages range from $2320 \mathrm{Ma}$ to $445 \mathrm{Ma}$ (Table 2). Around 80 percent of these analyses fall within an age range of 600-450 Ma, and 32 analyses yield a mean ${ }^{206} \mathrm{~Pb} /{ }^{238} \mathrm{U}$ age of $479.6 \pm 3.3 \mathrm{Ma}$ (Fig.7; Table 2), which is interpreted as the eruption age of this rhyolite sample (Fig.7). Precambrian zircons are rare.

Among these 80 concordant ages, 28 “younger" zircons (528-473 Ma) were chosen for in situ Hf isotope determination (Fig. 6). Compared with the highly variable ages of the sandstone or siltstone of the olistostrome matrix, the good concentration of the zircon crystallization ages of these zircons is in agreement with the narrow range of their ${ }^{176} \mathrm{Hf} /{ }^{177} \mathrm{Hf}$ ratios, varying from 0.282134 to 0.282517 (Table 3 ). All the analyses exhibit $\varepsilon_{\mathrm{Hf}}(\mathrm{t})$ values from +2.8 to -12.5 with a peak at -6.2 (Fig. 8). In this sample, the $\mathrm{T}_{\mathrm{DM}^{2}}{ }^{2}$ age ranges from 1.252.22 Ga with a cluster around $1830 \mathrm{Ma}$ (Fig. 9; Table 3).

\subsection{FR18-1}

Sample 13FR18-1 was collected from the Olistostrome sub-unit of the $\mathrm{S}^{\mathrm{t}}$-Georges-surLoire Unit, at Villemoissan (Fig. 2; Table 1), from a lens of coarse sandstone in the siltstone matrix (Fig. 4B). This coarse grained, poorly sorted, and angular or sub-angular sandstone has 
grain sizes around 0.3-1.5 mm with a concentration around 0.3-0.7 $\mathrm{mm}$ (Fig. 5C). The major detrital components are polycrystalline quartz, plagioclase, muscovite, and lithic fragments. In thin section, poorly rounded quartz grains represent nearly $70 \%$ of the detrital component. Plagioclase and muscovite are less than 1\%, and lithic fragments nearly 30\% (Fig. 5C). Zircons are mostly euhedral to subhedral, transparent and colorless, ranging from 30 to150 $\mu \mathrm{m}$ in length, and possess length to width ratios between $1.1: 1$ and $2.5: 1$. Concentric zoning is common in most crystals in CL images (Fig. 6).

Of the 100 analyses conducted on 100 zircons for this sample, 91 are concordant within uncertainties (Table 2). All zircons have Th/U ratios higher than 0.1. Concordant ages range from $2928 \mathrm{Ma}$ to $563 \mathrm{Ma}$, with one dominant peak at ca. $610 \mathrm{Ma}$ and one subordinate peak at ca. 1920 Ma. Three low backgrounds of dispersed ages fall into the Mesoarchean, Neoarchean, and Neoproterozoic (Fig. 7).

Twenty-eight dated zircons were chosen for in situ Hf isotope analyses among these 91 concordant analyses (Fig. 6; Table 3). The ${ }^{176} \mathrm{Hf} /{ }^{177} \mathrm{Hf}$ ratios vary from 0.280938 to 0.282335 . All the analyses have a $\varepsilon_{\mathrm{Hf}}(\mathrm{t})$ range from -20.4 to +1.7 with a peak at -5.5 (Fig. 8). The zircon $\mathrm{T}_{\mathrm{DM}}^{2}$ ranges from 3540 to $1700 \mathrm{Ma}$ with clusters around $2270 \mathrm{Ma}, 2710 \mathrm{Ma}$, and 3430 Ma (Fig. 9; Table 3).

\subsection{FR19}

Pervasively deformed muddy sandstone, sample 13FR19, is from Roche Ayrault (Fig. 2). Pressure solution cleavage is well developed in the outcrop and thin section (Figs. 4C and 5D). Fine grain quartz and mica constitute the major components of this sample (Fig. 5D). Zircon grains possess a relatively-round shape, ranging from 30 to $70 \mu \mathrm{m}$ in size (Fig. 6). 
A total of 69 analyses on 69 grains were obtained (Table 2). Among these analyses, 47 have concordant ages from 3109 Ma to 411 Ma. Two dominant age groups are documented at 720-400 Ma with a peak of ca. $600 \mathrm{Ma}$, and 3150-1550 Ma with a peak around $1975 \mathrm{Ma}$ (Fig.

7). This sample is the only one to yield detrital zircon ages younger than $450 \mathrm{Ma}(411 \pm 7 \mathrm{Ma}$, $411 \pm 8 \mathrm{Ma}$, and $426 \pm 6 \mathrm{Ma}$ ). $\mathrm{Th} / \mathrm{U}$ ratios of all zircons from this sample are close to or greater than 0.1 .

Because of the small size, only 19 dated zircons were measured for in situ Hf isotope determination from the 47 concordant analyses (Fig. 6; Table 3). The ${ }^{176} \mathrm{Hf} /{ }^{177} \mathrm{Hf}$ ratios vary from 0.281296 to 0.282565 . All $\varepsilon_{\mathrm{Hf}}(\mathrm{t})$ values range from +2.6 to -20.9 with two dominant peaks at -1.4 and -9.5 (Fig. 8). The $\mathrm{T}_{\mathrm{DM}^{2}}$ model ages vary from 1.31 to $3.20 \mathrm{Ga}$ with clusters around $2305 \mathrm{Ma}$, and $2870 \mathrm{Ma}$ (Fig. 9; Table 3).

\subsection{FR21}

To the west of Beaulieu-sur-Layon, the Pierre Bize quarry exposes a schistose-fine grain conglomerate with limestone and volcanic pebbles, which was chosen for detrital zircon analysis (Fig. 4D). A coarse-grained, poorly-sorted sandstone, with angularsubangular shaped, gravel-size clasts has a 0.3-1.5 mm grain size with $0.3-1.1 \mathrm{~mm}$ concentration (Fig. 5E). The main detrital components are polycrystalline quartz ( 40\%), plagioclase ( 2\%), muscovite (more than $20 \%$ ), and lithic fragments with carbonate cementation (Fig. 5E).

Zircon grains are mostly euhedral, transparent and colorless, ranging from 70 to $250 \mu \mathrm{m}$ in length, and with length to width ratios between 1.3:1 and 4:1. A total of 100 analyses of 100 grains from sample 13FR21 were obtained. 94 analyses are concordant within 
uncertainties (Table 2). Combined with CL images, the high $\mathrm{Th} / \mathrm{U}$ ratio (0.07-2.61) is indicative of magmatic origin (Fig. 6). Concordant ages range from $2690 \mathrm{Ma}$ to $399 \mathrm{Ma}$, with one dominant peak at $\sim 525 \mathrm{Ma}$. A few ages, between the Neoarchean and Paleoproterozoic are recorded with a small peak at $2630 \mathrm{Ma}$ (Fig. 7).

Thirty-five dated zircons were chosen for in situ Hf isotope analyses among these 94 concordant analyses (Fig. 6; Table 3). The Hf isotopic compositions reveal ${ }^{176} \mathrm{Hf} /{ }^{177} \mathrm{Hf}$ ratios varying from 0.281953 to 0.282718 . $\varepsilon_{\mathrm{Hf}}(\mathrm{t})$ values range from+9.6 to -18.7 with two dominant peaks at 2.7 and -3.4 (Fig. 8). More than half of the $\varepsilon_{\mathrm{Hf}}(\mathrm{t})$ values of Neoproterozoic to Cambrian zircons are positive (Table 3). The $\mathrm{T}_{\mathrm{DM}^{2}}$ model ages range from 3540 to $1700 \mathrm{Ma}$ with a cluster around 1285 Ma (Fig. 9; Table 3).

\section{$6.6 \mathrm{RO} 2$}

Sample RO2 is a fine-grained siltstone in the clastic matrix of the olistostrome sub-unit, taken from the $S^{\mathrm{t}}$-Georges-sur-Loire Unit near Rochefort-sur-Loire (Figs. 2, 4E; Table 1). This siltstone is fine grained, poorly-sorted, and angular. Its grain size is around 0.1-0.7 mm with a concentration around 0.2-0.4 mm (Fig. 5F). The major detrital components are quartz, muscovite, and sedimentary lithic fragments. In thin section, poorly rounded quartz represents $78 \%$ of the detrital component, and muscovite comprises about $20 \%$ (Fig. 5F).

Zircon grain size ranges from 40 to $160 \mu \mathrm{m}$ with length to width ratios between $1: 1$ and 2:1 (Fig. 6). A total of 185 analyses of 185 grains were obtained for this sample (Table 2). Among these analyses, 100 concordant ages range from $3300 \mathrm{Ma}$ to $447 \mathrm{Ma}$ with $\mathrm{Th} / \mathrm{U}$ ratios close to or higher than 0.1 (Table 2). Two dominant age groups occur at 720-460 Ma with a 
peak of ca. $590 \mathrm{Ma}$, and 2200-1900 Ma with two peaks around 2130 Ma and $1910 \mathrm{Ma}$. A low background of dispersed ages ranges from 3300 Ma to $2500 \mathrm{Ma}$ (Fig. 7).

Forty dated zircons were measured for Hf isotope determination among these 117 concordant analyses (Fig. 6; Table 3). ${ }^{176} \mathrm{Hf} /{ }^{177} \mathrm{Hf}$ ratios vary from 0.280543 to 0.282664 . All the analyses reveal a $\varepsilon_{\mathrm{Hf}}(\mathrm{t})$ range from -31.3 to +30.9 with two dominant peaks at +6.5 and 1.7 (Fig. 8). $\mathrm{T}_{\mathrm{DM}}^{2}$ model ages range from 0.63 to $3.95 \mathrm{Ga}$ with clusters around $2970 \mathrm{Ma}$, and 2255 Ma (Fig. 9; Table 3).

\subsection{OUT}

The sandstone sample OUT was collected to the NW of the $\mathrm{S}^{\mathrm{t}}$-Georges-sur-Loire Unit (Fig. 2; Table 1). In the fine-grained, poorly sorted, and angular matrix-supported sandstone, grain size is around 0.1-0.5 mm with a concentration around 0.2-0.4 $\mathrm{mm}$ (Fig. 5G). The major detrital components are quartz, muscovite and sedimentary lithic fragments. In thin section, poorly rounded to subangular gravel-size quartz represents $92 \%$ of the detrital component, muscovite is less than $1 \%$, and lithic fragmented about $7 \%$ (Fig. 5G). As a fine grained sandstone, the zircon grains range from 50 to $250 \mu \mathrm{m}$ with length to width ratio between 1:1.2 and 2.5:1 (Fig. 6).

A total of 100 analyses from 100 grains were obtained for this sample (Table 2). Among these analyses, 71 are concordant within uncertainties, ranging from $2998 \mathrm{Ma}$ to $299 \mathrm{Ma}$. Only one grain is younger than $480 \mathrm{Ma}$ (Fig. 7). The Th/U ratios of almost all zircons are greater than 0.1, except for one grain (Table 2). Two dominant age groups are at 680-490 Ma with a peak of $595 \mathrm{Ma}$, and 2200-1890 Ma with a peak around $1930 \mathrm{Ma}$ (Fig. 7). 
Seventy-one dated zircons were chosen for in situ Hf isotope analyses among these 71 concordant analyses (Fig. 6; Table 3). The ${ }^{176} \mathrm{Hf} /{ }^{177} \mathrm{Hf}$ ratio varies from 0.280793 to 0.282602 . $\varepsilon_{\mathrm{Hf}}(\mathrm{t})$ values range from +24.1 to -25.8 with two dominant peaks at -4.0 and -7.8 and also with two subordinate peaks at 7.6 and -16.7 (Fig. 8). The $\mathrm{T}_{\mathrm{DM}^{2}}$ model ages range from 0.98 to 3.62 Ga with clusters around $1200 \mathrm{Ma}, 1870 \mathrm{Ma}$, and $2945 \mathrm{Ma}$ (Fig. 9; Table 3).

\section{$6.8 \mathrm{MB} 2$}

Sample MB2 is a deformed sandstone from the matrix of the olistostrome subunit of the $\mathrm{S}^{\mathrm{t}}$-Georges-sur-Loire Unit, Moulin de Beaupreau (Fig. 2; Table 1). In this fine-grained, angular to subangular sandstone, grain size is around $0.1-0.7 \mathrm{~mm}$ with a concentration around 0.3-0.5 mm (Fig. 5H). The major detrital components are quartz, muscovite and sedimentary lithic fragments. In thin section, deformed quartz grains represent $78 \%$ of the detrital component, muscovite and sedimentary lithic fragments are more than $7 \%$, and $15 \%$, respectively (Fig. 5H). The zircon grains range from 70 to $150 \mu \mathrm{m}$ with length-to-width ratios between 1:1 and 1.7:1 (Fig. 6).

A total of 100 analyses on 100 grains were carried out (Table 2), and 74 are concordant, ranging in age from $2717 \mathrm{Ma}$ to $280 \mathrm{Ma}$. The $\mathrm{Th} / \mathrm{U}$ ratios of all zircons are greater than 0.1 (Table 2). Two dominant age groups are documented at 680-520 Ma with a peak of ca. 615 Ma, and 2230-1855 Ma with a peak around ca. 1940 Ma (Fig. 7). Two grains are younger than 470 Ma (Fig. 7).

Hf isotope determination of seventy-two dated zircons from 74 concordant analyses shows ${ }^{176} \mathrm{Hf} /{ }^{177} \mathrm{Hf}$ ratios vary from 0.280781 to 0.282567 (Fig. 6; Table 3). All the analyses exhibit $\varepsilon_{\mathrm{Hf}}(\mathrm{t})$ values from -27.4 to +8.0 with one dominant peak at -5.1 . Several subordinate 
peaks can be identified at $+6.4,-10.5,-16.2$, and -22.9 respectively (Fig. 8 ). The $\mathrm{T}_{\mathrm{DM}}{ }^{2}$ range from 1.11 to $4.29 \mathrm{Ga}$ with clusters around $1810 \mathrm{Ma}, 2300 \mathrm{Ma}$, and $2830 \mathrm{Ma}$ (Fig. 9; Table 3).

\section{Provenance of the detrital zircons in St-Georges-sur-Loire Unit}

The $\mathrm{S}^{\mathrm{t}}$-Georges-sur-Loire Unit has been interpreted as a remnant back-arc basin deformed during the Variscan orogeny (Carpenter et al., 1982; Cabanis, 1986; Cartier and Faure, 2004; Fig. 1). Most of the sedimentary and magmatic blocks enclosed in a clastic matrix are interpreted as olistoliths derived from a southern area called the "missing domain", presently exposed as the Variscan Mauges Nappe composed of Cadomian rocks and its Early Paleozoic sedimentary cover (Dubreuil, 1986; Ducassou et al., 2009, 2011, 2014). The "missing domain" is interpreted to have been subducted below Armorica during the Early Devonian (Faure et al., 2008) or the Middle Devonian Variscan collision (Ducassou et al., 2014; Paquette et al., 2017). Eight samples have been analyzed for zircon geochronology in the $\mathrm{S}^{\mathrm{t}}$-Georges-sur-Loire Unit (Fig. 2); seven samples of them are sandstone or siltstone in the olistostrome matrix, and one is a rhyolitic block as an olistolith. Our new detrital zircon U-Pb ages exhibit age peaks from the Neoarchean to Early Paleozoic at $2650 \mathrm{Ma}, 2065 \mathrm{Ma}, 1910$ Ma, 625 Ma, 595 Ma and 490 Ma (Fig. 11A). Statistically, the Neoproterozoic (Cadomian) age cluster is the dominant one that contains nearly half $(45.2 \pm 2.8 \%)$ of all zircon ages (Fig. 11A), similar to the detrital zircon age distribution from the Late Paleozoic sedimentary sequences of the Montagne Noire area of French Massif Central, Corsica and West Avalonia

(Ganderia and Notre Dame Subzone) (Fig. 13; Henderson et al., 2016, 2018; Lin et al., 2016; Avigad et al., 2018). A secondary Paleoproterozoic age peak (with $27.4 \pm 1.7 \%$ of measured zircon grains) range from $2100 \mathrm{Ma}$ to $1900 \mathrm{Ma}$ (Lin et al., 2016). Nearly $20 \%$ of the 
analytical results constitute a 490 Ma peak that was related to Late Cambrian rifting

(Fig.11A).

\subsection{Provenance of Paleozoic zircons}

Early Paleozoic ages around $490 \mathrm{Ma}$ and $525 \mathrm{Ma}$ (Early Cambrian) were identified in the contributions of the 13FR13 Epiré rhyolite, and in the 13FR21 Pierre Bize sandstone (Figs. 7, 11A). Cambrian-Ordovician alkaline magmatism is widespread in the pre-Mesozoic European basement. It can be divided into two distinct rifting events, a Cambrian one at ca. $520 \mathrm{Ma}$ represents a failed rift stage, and an Early-Middle Ordovician one at ca. 480-460 Ma that corresponds to the main stage responsible for the break-up of the Pannotia megacontinent and the opening of the Early Paleozoic oceans (e.g. Matte, 2001; Winchester et al., 2002; Linneman et al., 2004, 2007; Murphy et al., 2006). In the Armorican Massif, Cambrian magmatism is well recorded in the North Armorican Domain (Doré, 1994; Le Gall, 1993), and in the South Armorican Domain, for example, the protolith of the migmatitic orthogneisses from the Champtoceaux Unit (Cocherie et al., 2005), or the Cambrian rhyolite in the Mauges Nappe (Thiéblemont et al., 2001). Early Ordovician magmatism is also well preserved in the Armorican Massif (Auvray et al., 1980; Paquette et al., 1984, 2017; Janjou et al., 1998; Ballèvre et al., 2002, 2012; Galerne et al., 2006; Diot et al., 2007; Casas and Murphy, 2018), and the French Massif Central (e.g. Alexandrov et al., 2001; Melleton et al., 2010; Lin et al., 2016). In contrast, Late Paleozoic ages are rarely recorded in the detrital zircon populations (Figs. 7 and 11A; Table 2), but limited zircon grains in sample 13FR19 demonstrate three ages at $411 \pm 7 \mathrm{Ma}, 411 \pm 8 \mathrm{Ma}$, and 426 $\pm 6 \mathrm{Ma}$, respectively. Although these results may not be sufficient to directly prove the existence of the Variscan magmatic arc as previously proposed (Table 2; Dubreuil, 1986; Cartier and Faure, 2004; Faure et al., 2008; 
Ducassou et al., 2014), however, considering detrital mica ${ }^{40} \mathrm{Ar} /{ }^{39} \mathrm{Ar}$ ages at $430-425 \mathrm{Ma}$ reported in the Ancenis Basin, and detrital zircon $\mathrm{U}-\mathrm{Pb}$ ages from our samples, it implies a Variscan thermo-tectonic event recorded in the sediments of the Ancenis Basin and the $\mathrm{S}^{\mathrm{t}}$ Georges-sur-Loire Unit (Ducassou et al., 2014; Fig. 13; Table 2).

A possible geodynamic reconstruction of the Silurian-Devonian geodynamic setting is proposed in Fig. 14. During the Silurian-Early Devonian (ca 440-400 Ma,) the MedioEuropean Ocean located between Armorica and Gondwana was closed by north-directed subduction (Faure et al., 2008). The southern continental margin of Armorica was a magmatic arc-back arc system. Detrital zircon analyses from the $\mathrm{S}^{\mathrm{t}}$-Georges-sur-Loire Unit document Early Paleozoic, Neoproterozoic and Paleoproterozoic-Archean sources. Presently, the Icartian basement, Cadomian orogenic belt and Early Paleozoic magmatic rocks are exposed in North-and Central Armorica, and to a lesser extent, in the basement of the magmatic arc that is only preserved in the Mauges nappe. Therefore, both northern and southern provenances must be considered.

Nearly $10 \%$ of the detrital zircons from the $\mathrm{S}^{\mathrm{t}}$-Georges-sur-Loire yield Ediacaran-Early Cambrian ages, around $540 \mathrm{Ma}$. These ages correspond to that of peraluminous magmatism of the Mancellian granites in the Fougères Unit, and migmatization in the $\mathrm{S}^{\mathrm{t}}$-Malo and Guingamp Units (Fig. 1). Both rock types record late Cadomian crustal melting (Egal et al., 1996; Chantraine et al., 2001).

Detrital zircons clustering between 480 and 560 Ma have mostly negative $\varepsilon_{H f}(t)$ values in agreement with old continental crustal sources. A few positive $\varepsilon_{\mathrm{Hf}}(\mathrm{t})$ values is indicative of an addition of juvenile material from a depleted mantle during the Cambrian and Ordovician rifting (Kröner et al., 2014; Fig. 13). The late-orogenic Cadomian magmatism at 550-540 Ma is typical of crustal reworking (Fig. 9; Table 3). The Early to Middle Cambrian (ca 520 Ma), 
and the Early to Middle Ordovician (490-460 Ma) rifting events might have been associated with a crustal reworking along with a minor mantle input (Auvray et al., 1980; Paquette et al., 1984; Doré, 1994; Le Gall, 1993; Ballèvre et al., 2002; Linneman et al., 2004, 2007; Cocherie et al., 2005; Murphy et al., 2006).

\subsection{Provenance of Neoproterozoic zircons}

A main Ediacaran peak, between $625 \mathrm{Ma}$ and $595 \mathrm{Ma}$, is prominently marked in most samples (Figs. 7 and 11A), indicating that the provenance of the $\mathrm{S}^{\mathrm{t}}$-Georges-sur-Loire Unit material may be from a Neoproterozoic basement, such as the Cadomian belt exposed in the St- Brieuc and Trégor Units of the north Armorican massif (Chantraine et al., 2001). There, a large number of plutons yield Ediacaran ages at ca. 615-570 Ma (Adams, 1976; Ruffet et al., 1990, 1991; Dallmeyer et al., 1991; Egal et al., 1996). Thus, eroded plutons and volcanic rocks might have a significant role as sources providing detrital zircons to the $\mathrm{S}^{\mathrm{t}}$-Georges-surLoire Unit. In the Pierre Bize coarse sandstone (sample 13FR21), the 525 Ma peak suggest a possible source from plutons equivalent to the Thouars microgranite and the Mouclerie granodiorite that crop out in the Mauges Nappe of the S. Armorican Domain (Fig. 1; Thiéblemont et al., 2001), and the Mauges Nappe represents the only exposure of the "missing domain" at the south $\mathrm{S}^{\mathrm{t}}$-Georges-sur-Loire Unit before the Variscan orogeny (Dubreuil, 1986; Cartier and Faure, 2004; Faure et al., 2008). Thus the provenance of the Cadomian zircons of the matrix of the $\mathrm{S}^{\mathrm{t}}$-Georges-sur-Loire olistostrome might be supplied from the Neoproterozoic basement of the Mauges Nappe and/or its equivalent, or alternatively, from the Cadomian orogen exposed in the Central-North Armorican Domain (Fig. 13). Although a northern provenance cannot be ruled out, a southern origin would be in better 
agreement with the sedimentological features of the olistoliths (Dubreuil, 1986; Cartier and Faure, 2004).

7.3. Inherited Neoarchean and Paleoproterozoic zircons from the Icartian basement

One peak at 2090-2040 Ma is obtained from samples 13FR12 and 13FR21 (Fig. 11A). This age group well recorded in the Trégor Unit of the North Armorican Domain characterizes the Icartian basement (Fig. 1; Calvez and Vidal, 1978), which is an equivalent of the Eburnean basement of the West African Craton (Allègre and Ben Othman, 1980). Similar ages are also discovered from inherited zircons in the Late Carboniferous (320-300 Ma) peraluminous granites (Ballouard et al., 2018). The notable peak at ca. $1910 \mathrm{Ma}$, marked in most samples, indicate a major source from an ancient Icartian continental crust. In the Carboniferous Ancenis Basin deposited on the Mauges Unit, and in the Late Carboniferous coal-bearing basin, a background of dispersed ages ranges from Neoarchean to Paleoproterozoic with three peaks about $2650 \mathrm{Ma}, 2065 \mathrm{Ma}$ and $1910 \mathrm{Ma}$ (Fig. 11B; Ducassou et al., 2014). Thus, these grains might either be reworked in the vicinity of the $\mathrm{S}^{\mathrm{t}}$ Georges-sur-Loire Unit, or directly supplied from a northern Icartian source.

Recycled zircons from Archean-Paleoproterozoic magmatic and metamorphic sources are documented in the Iberian massif (Fernández-Suárez et al., 2000, 2002, 2003; Martínez Catalán et al., 2004, 2008, 2009), the French Massif Central (Lin et al., 2016), Cadomian metasedimentary rocks in Corsica (Avigad et al., 2018), and Central European Variscan Massifs (Friedl et al., 2000; Linneman et al., 2004). 


\subsection{Interpretation of the Hf isotopes}

Hf isotopic data show the predominance of crustal vs. mantle-derived sources in felsic to intermediate magmatism. As indicated by Condie et al. (2009), $75-80 \%$ of juvenile continental crust had been extracted from the mantle during the Archean and the Paleoproterozoic. Detrital zircon $\varepsilon_{\mathrm{Hf}}(\mathrm{t})$ values in the $\mathrm{S}^{\mathrm{t}}$-Georges-sur-Loire Unit record a relatively wide variation from -12 to +4 , with a peak at -5 (Fig. 10A). Hf crustal model ages $\left(\mathrm{T}_{\mathrm{DM}^{2}}{ }^{2}\right.$ ) range from 3.5 to $1.0 \mathrm{Ga}$, with peaks at $\sim 1.28,1.98,2.20$, and $2.86 \mathrm{Ga}$ (Fig. 10B). The Hf isotopic composition for detrital zircons clustering between 480 and 650 Ma demonstrate a relatively large range of $\varepsilon_{\mathrm{Hf}}(\mathrm{t})$ values from -25 to +10 (Fig. 12B). Despite two anomalous $\varepsilon_{\mathrm{Hf}}$ (t) values higher than the depleted mantle line, only a few points reach the depleted mantle line, and points with $\varepsilon_{\mathrm{Hf}}(\mathrm{t})$ value lower than -20 are also rare (Figs. 10A and 12A). Combined with the calculated $\mathrm{T}_{\mathrm{DM}^{2}}$ ages for most of the Phanerozoic zircons that range from $1.2 \mathrm{Ga}$ to 3.0 Ga (Figs. 10B and 12A), these results are comparable with the previous geochemical studies on the mafic complexes that range in age from ca. $400 \mathrm{Ma}$ to $330 \mathrm{Ma}$, which are characterized by high $\varepsilon_{\mathrm{Nd}}$ values related to the lithosphere of the Rheic Ocean (Murphy et al., 2011, 2014).

\section{Conclusion}

Detrital zircon ages of the clastic matrix in the $\mathrm{S}^{\mathrm{t}}$-Georges-sur-Loire olistostrome display predominant Neoproterozoic to Early Paleozoic (625-490 Ma) populations. These zircons are related to Cambrian aborted rifting at ca. $525 \mathrm{Ma}$, and Early Ordovician rifting around 480-470 Ma that separated the Armorica microcontinent from Gondwana. Poorly constrained Silurian to Early Devonian magmatic rocks that yield a whole rock $\mathrm{Rb}-\mathrm{Sr}$ age at ca. $400 \pm 24 \mathrm{Ma}$ (Carpenter et al., 1982) are found as olistoliths in the $\mathrm{S}^{\mathrm{t}}$-Georges-sur-Loire 
Unit. However, this age peak is not documented in our study, as well as in the late Ancenis and Coal basins. On the contrary, the Neoproterozoic age population corresponds well to the Cadomian orogeny exposed on both sides of the $S^{t}$-Georges-sur-Loire Unit. A southern provenance, from the Mauges Nappe and the "missing domain" is in better agreement with sedimentological data (e.g. Dubreuil, 1986; Cartier et al., 2001), however a northern one supplied by the Cadomian belt of the North-Central Armorican domain cannot be ruled out. Most of the grains record negative $\mathrm{Hf}$ isotopic values, indicating that crustal melting played a major role in the evolution of the Armorica microcontinent. Positive $\varepsilon_{\mathrm{Hf}}(\mathrm{t})$ values are related to the Neoproterozoic Cadomian orogeny in which subduction-related juvenile magma are recognized.

\section{Acknowledgments}

Brendan Murphy and Michel Ballèvre are deeply acknowledged for their constructive scientific and formal remarks to improve an early draft of this paper. Prof. Mark Allen is thanked for his help to polish the English of the final version. This work is supported by the National Nature Science Foundation of China (91855212, 91755205, and 41472193) and the National Key R\&D Program of China (Grant Numbers 2016YFC0600401, 2016YFC0600102).

\section{References}

Adams, C. J. D., 1976. Geochronology of the Channel Islands and adjacent French mainland. Geol. Soc. London 142, 233-250.

Alexandrov, P., Floćh, J.P., Cuney, M., Cheilletz, A., 2001. Datation U-Pb à la microsonde ionique des zircons de l'unité supérieure de gneiss dans le Sud Limousin, Massif Central. C. R. Acad. Sci. 332, 625632.

Allègre, C., Ben Othman, D., 1980. Nd-Sr isotopic relationship in granitoid rocks and continental crust 
development: a chemical approach to orogenesis. Nature 268, 335-342.

Andersen, T., 2002. Correction of common lead in U-Pb analyses that do not report 204Pb. Chem. Geol. 192, 59-79.

Auvray, B., Macé, J., Vidal, P. Van Der Voo, R., 1980. Rb-Sr dating of the Plouézec volcanics, N Brittany: implications for the age of the red beds ("séries rouges") in the northern Armorican Massif. J. Geol. Soc. London 137, 207-210.

Avigad, D., Rossi, Ph., Gerdes, A., Abbo, A., 2018. Cadomian metasediments and Ordovician sandstone from Corsica: detrital zircon U-Pb-Hf constrains on their provenance and paleogeography. Int. J. Earth Sci. $107,2803-2818$.

Ballèvre, M., Capdevila, R., Guerrot, C., Peucat, J.J., 2002. Discovery of an alkaline orthogneiss in the eclogite-bearing Cellier Unit (Champtoceaux Complex, Armorican Massif): a new witness of the Ordovician rifting. C. R. Geoscience 334, 301-311.

Ballèvre, M., Bosse, V., Ducassou, C., Pitra, P., 2009. Palaeozoic history of the Armorican Massif: Models for the tectonic evolution of the suture zones. C. R. Geoscience 341, 174-201.

Ballèvre, M., Fourcade, S., Capdevila, R., Peucat, J.J., Cocherie, A., Fanning, C. M., 2012. Geochronology and geochemistry of Ordovician felsic volcanism in the Southern Armorican Massif (Variscan belt, France): implications for the breack up of Gondwana. Gond. Res. 21, 1019-1036.

Ballouard, C., Poujol, M., Zeh, A., 2018. Multiple crust reworking in the French Armorican Variscan belt: Implication for the genesis of uranium-fertile leucogranites. Int. J. Earth Sci. 107, 2317-2336.

Berthé, D., Choukroune, P., Jegouzo, P., 1979. Orthogneiss, mylonite and non coaxial deformation of granites: the example of the South Armorican Shear Zone. J. of Struct. Geol. 1, 31-42.

Bruguier, O., Lancelot, J.R., Malavieille, J., 1997. U-Pb dating on single detrital zircon grains from the Triassic Songpan-Ganze flysch (Central China): provenance and tectonic correlations. Earth Planet. Sci. Lett. 152, 217-231.

Cabanis, B., 1986. Identification des séries magmatiques dans les socles métamorphiques sur la base de critères géologiques, pétrographiques et géochimiques. Exemples d'application dans le Massif armoricain. Implications géodynamiques. Contribution à une méthode d'étude basée sur les éléments traces. Thèse d’Etat, Université Pierre et Marie Curie, Paris pp. 1-682.

Cai, F.L., Ding, L., Laskowski, A.K., Kapp, P., Wang, H., Xu, Q., Zhang, L., 2016. Late Triassic paleogeographic reconstruction along the Neo-Tethyan Ocean margins, southern Tibet. Earth Planet. Sci. Lett. 435, 105-114.

Calvez, J.Y., Vidal, P., 1978. Two billion years old relicts in the Hercynian Belt of Western Europe. Contrib. Mineral. Petrol. 65, 395-399.

Carpenter, M., Peucat, J.J., Pivette, B., 1982. Geochemical and geochronological characteristics of 
Palaeozoic volcanism in the Saint-Georges-sur-Loire synclinorium (S. Armorican Massif). Evidence for pre-hercynian tectonic evolution. Bull. Bur. Rech. Géol. Min. 21, 63-79.

Cartier, C., Faure, M., 2004. Structure and Geodynamic evolution of the Gondwana-Armorica boundary in the Ligerian domain (Armorican massif, France). Int. J. Earth Sci. 93, 945-958.

Cartier, C., Faure, M., Lardeux, H., 2001. The Hercynian orogeny in the South Armorican Massif (France): Rifting and welding of continental stripes. Terra Nova 14, 143-149.

Casas, J., Murphy, B., 2018. Unfolding the arc: the use of pre-orogenic constraints to assess the evolution of the Variscan belt in Western Europe. Tectonophysics 736, 47-61.

Cavet, P., Arnaud, A., Blaise, J., Gruet, M., Lardeux, H., Rivière, L.M., Chauris, L., Guignes, J., 1970. Notice explicative de la carte géologique de la France (1/50000), feuille Chalonnes-sur-Loire (453), BRGM, Orléans. (32 pp.).

Cavet, P., Arnaud, A., Blaise, J., Gruet, M., Lardeux, H., Marchand, J. Nicolas, A., Rivière, Rossignol, J.C., Arnaud, A., 1978. Notice explicative de la, carte géologique de la. France (1/50000), feuille d'Ancenis (452), BRGM, Orléans. (56 pp.).

Cawood, P.A., Johnson, M.R.W., Nemchin, A.A., 2007. Early Palaeozoic orogenesis along the Indian margin of Gondwana: tectonic response to Gondwana assembly. Earth Planet. Sci. Lett. 255, 70-84.

Cawood, P.A., Hawkesworth, C.J., Dhuime, B., 2012. Detrital zircon record and tectonic setting. Geology $40,875-878$.

Chantraine, J., Egal, E., Thiéblement, D., Le Goff, E., Guerrot, C., Ballèvre, M., Guennoc, P., 2001. The Cadomian active margin (North Armorican Massif, France): a segment of the North Atlantic Panafrican belt. Tectonophysics 331, 1-18.

Chu, Y., Lin W., Faure M., Wang Q., 2016. Detrital zircon U-Pb ages and Hf isotopic constraints on the terrigenous sediments of the Western Alps and their paleogeographic implications, Tectonics 35. http://dx.doi.org/10.1002/2016TC004276.

Cocherie, A., Bé Mezème, E., Legendre, O., Fanning, C.M., Faure, M., Rossi, M., 2005. Electron microprobe dating as a tool for determining the closure of the Th-U-Pb system in migmatitic monazites. Am. Mineral 90, 607-618.

Condie, K.C., Belousova, E., Griffin,W.L., Sircombe, K.N., 2009. Granitoid events in space and time: constraints from igneous and detrital zircon age spectra. Gond. Res. 15, 228-242.

Dallmeyer, R.D., Strachan, R.A., D'Lemos, R.S., 1991. Chronology of Cadomian tectonothermal activity in the Baie de Saint-Brieuc (North Brittany), France: evidence from ${ }^{40} \mathrm{Ar} /{ }^{39} \mathrm{Ar}$ mineral ages. Can. J. of Earth Sci. $28,762-773$.

Dickinson, W., Gehrels, G., 2009. Use of U-Pb ages of detrital zircons to infer maximum depositional ages of strata: A test against a Colorado Plateau Mesozoic database. Earth Planet. Sci. Lett. 288, 115-125. 
Diot, H., Blaise, J., 1978. Etude structurale dans le Précambrien et le Paléozoïque de la partie méridionale du domaine ligérien (SE du Massif Armoricain): Mauges, synclinal d'Ancenis et Sillon Houiller de basse Loire. Bull Soc Géol Miné. Bretagne C, X, 31-50.

Diot, H., Femenia,s O., Moreau, C., Gaufriau, A., Roy, C., Karnay, G., 2007. Notice explicative de la carte géologique de France au 1:50000, feuille Fontenay-le-Comte. BRGM, Orléans, pp.96.

Dissler, E., Doré, F., Dupret, L., Gresselin, F., Le Gall, J., 1988. L'évolution géodynamique cadomienne du Nord-est du Massif armoricain. Bull. Soc. Geol. Fr. 4, 801-814.

Doré, F., 1994. Cambrian of the Armorican Massif. In: Keppie, J. D. (ed.) Pre-Mesozoic Geology in France and Related Areas. Springer, Berlin, 136-141.

Dubreuil, M., 1986. Evolution géodynamique du Paléozoïque Ligérien (Massif Armoricain), Thèse d'Etat, Université de Nantes, pp. 1-258.

Ducassou, C., Ballèvre, M., Lardeux, H., Robin, C., 2011. Evidence for pre-orogenic, Early Devonian rifting in the Variscan belt: stratigraphy and structure of the Palaeozoic cover of the Mauges Unit (Upper Allochthon, Armorican massif, France). Int. J. Earth Sci. 100, 1451-1476.

Ducassou, C., Strullu-Derrien, C., Ballèvre, M., Dabard, M.P., Gerrienne, P., Lardeux, H., Robin, C., 2009. Age and depositional environment of the Sainte-Anne Formation (Armorican Massif, France) : the oldest (Emsian) evidence for mountain erosion in the Variscan belt. Bull.de la Soc. Géol. de Fr. 180, 529-544.

Ducassou, C., Poujol, M., Ruffet, G., Bruguier, O., Ballèvre, M., 2014. Relief variation and erosion of the Variscan belt: detrital geochronology of the Palaeozoic sediments from the Mauges Unit (Armorican Massif, France). In: Schulmann, K., Martínez Catalán, J.R., Lardeaux, J.M., Janouscek, V. and Oggiano, G. (eds). The Variscan Orogeny: Extent, Timescale and the Formation of the European Crust. Geol. Soc. London, Spec. Publ. 405, 137-167.

Egal, E., Guerrot, C., Le Goff, E., Thiéblemont, T., Chantraine, J., 1996. The Cadomina Orogeny revisited in Northern Brittany (France). In: Nance, R.D. and Thompson, M.D. (eds) Avalonian and Related PeriGondwanan Terranes of the Circum- North Atlantic. Geol. Soc. of Am. Spec. Papers, 304, 281-319.

Faure, M., Bé Mézème, E., Cocherie, A., Rossi, P., Chemenda, A., Boutelier, D., 2008. Devonian geodynamic evolution of the Variscan Belt, insights from the French Massif Central and Massif Armoricain, Tectonics 27, TC2008, http://dx.doi.org/10.1029/2007TC002115.

Faure, M., Bé Mézème, E., Duguet, M., Cartier, C., Talbot, J.Y., 2005. Paleozoic tectonic evolution of Medio-Europa from the example of the French Massif Central and Massif Armoricain. J. of Virtual Explorer Electr. edition ISSN 1441-8142, 19 paper 5, 1-21.

Faure, M., Sommers, C., Melleton, J., Cocherie, A., Lautout, O., 2010. The Léon Domain (French Massif Armoricain): a westward extension of the Mid-German Crystalline Rise. Structural and geochronological insights. Int. J. Earth Sci. 99, 65-81. DOI: 10.1007/s00531-008-0360x.

Fedo, C.M., Sircombe, K.N., Rainbird, R.H., 2003. Detrital zircon analysis of the sedimentary record. In: 
Hanchar, J.M., Hoskin, P.W.O. (Eds.), Zircon. Rev. in Min. and Geochem. 53, pp. 277-303.

Fernández-Suárez, J., Díaz-García, F., Jeffries, T.E., Arenas, R., Abati, J., 2003. Constraints on the provenance of the uppermost allochtonous terrane of the NW Iberian Massif: inferences from detrital zircon U-Pb age. Terra Nova 15, 138-144.

Fernández-Suárez, J., Gutiérrez-Alonso, G., Jenner, G.A., Tubrett, M.N., 2000. News ideas on the Proterozoic-Early Palaeozoic evolution of NW Iberia: insights from U-Pb detrital zircon ages. Precambrian Res. 102, 185-206.

Fernández-Suárez, J., Gutiérrez-Alonso, G., Jeffries, T.E., 2002. The importance of along-margin terrane transport in northern Gondwana: insights from detrital zircon parentage in Neoproterozoic rocks from Iberia and Brittany. Earth Planet. Sci. Lett. 204, 75-88.

Friedl, G., Finger, F., McNaughton, N.J., Fletcher, I.R., 2000. Deducing the ancestry of terranes: SHRIMP evidence for South America-derived Gondwana fragments in central Europe. Geology 28, 1035-1038.

Galerne, C., Caroff, M., Rolet, J., Le Gall, B., 2006. Magma-sediment mingling in an Ordovician rift basin: The Plouérec-Plourivo half-graben, Armorican Massif, France. Journal of Volcanology and Geothermal Research 155, 164-178.

Gehrels, G., Kapp, P., DeCelles, P., Pullen, A., Blakey, R., Weislogel, A., Ding, L., Guynn, J., Martin, A., McQuarrie, N., Yin, A., 2011. Detrital zircon geochronology of pre-tertiary strata in the Tibetan-Himalayan orogen. Tectonics 30, TC5016. http://dx.doi.org/10.1029/2011TC002868.

Gehrels, G.E., Dickinson, W.R., 1995. Detrital zircon provenance of Cambrian to Triassic miogeoclinal and eugeoclinal strata in Nevada. Am. J. of Sci. 295, 18-48.

Gougeon, R., Néraudeau, D., Dabard, M.P., Pierson-Wickmann, A.C., Polette, F., Poujol, M. Saint-Martin, J.P. 2018. Trace Fossils from the Brioverian (Ediacaran-Fortunian) in Brittany (NW France). Ichnos 25 , 11-24.

Graviou, P., Peucat, JJ, Auvray, B., Vidal, P., 1988. The Cadomian orogeny in the northern Armorican Massif-petrological and geochronological constraints on a geodynamic model. Hercynica IV, 1-13.

Griffin, W.L., Pearson, N.J., Belousova, E.A., Jackson, S.E., van Achterbergh E., O’Reilly, S.Y., Shee, S.R., 2000. The Hf isotope composition of cratonic mantle: LAM-MC-ICPMS analyses of zircon megacrysts in kimberlites. Geochim. et Cosmochimi. Acta 64, 133-147.

Guerrot, C., Peucat, J.J. 1990. U-Pb geochronology of the Late Proterozoic Cadomian orogeny in the northern Armorican Massif, France. In D’lemnos, R.S. Strachan, R.A. Topley, C.G. (Eds), the Cadomian orogeny. Geol. Soc. London, Spec. Publ. 51, 13-26.

Henderson, B. J., Collins, W. J., Murphy, J.B., Alonso, G., Handa, M., 2016. Gondwanan basement terranes of the Variscan-Appalachian orogen: Baltican, Sahara and West African hafnium isotopic fingerprints in Avalonian, Iberia and Armorican Terranes. Tectonophysics 681, 278-304. 
Henderson, B. J., Collins, W. J., Murphy, J.B., Hand, M., 2018. A hafnium isotopic record of magmatic arcs and continental growth in the Iapetus Ocean: The contrasting evolution of Ganderia and the periLaurentian margin. Gond. Res. 58, 141-160.

Janjou, D., Lardeux, H., Chantraine, J., Callier, L., Etienne, H., 1998. Notice explicative de la carte géologique de la France (1/50000), feuille de Segré (422), BRGM, Orléans.

Jégouzo, P., 1980. The South-Armorican Shear Zone. J. of Struct. Geol. 2, 39-47.

Kröner, A., Kovach, V., Belousova, E., Hegner, E., Armstrong, R., Dolgopolova, A., Seltmann, R., Alexeiev, D.V., Hoffmann, J.E., Wong, J., Sun, M., Cai, K., Wang, T., Tong, Y., Wilde, S.A., Degtyarev, K.E., Rytsk, E., 2014. Reassessment of continental growth during the accretionary history of the Central Asian Orogenic Belt. Gond. Res. 25, 103-125.

Lardeux, H., Cavet, P., 1994. Paleozoic of the Ligerian domain. In: Keppie JD (ed). Pre-Mesozoic Geology in France and related areas. Springer, Berlin Heidelberg New York, pp. 152-156.

Le Gall, J., 1993. Pétrogenèse des magmas andésitiques et ignimbritiques et leur signification dans l'évolution géodynamique cadomienne. Reconstitution des dynamismes éruptifs d'une province paléovolcanique: l'exemple du graben cambrien du Maine (Est du Massif Armoricain). Mémoires de Géosciences Rennes 52, pp. 370.

Lin, W., Faure, M., Li, X., Chu, Y., Ji, W., Xue, Z., 2016. Detrital zircon age distribution from Devonian and Carboniferous sandstone in the Southern Variscan Fold-and-Thrust belt (Montagne Noire, French Massif Central), and their bearings on the Variscan belt evolution. Tectonophysics 677-678, 1-33.

Lin, W., Rossi, P., Faure, M., Li, X., Ji, W., Chu, Y., 2018. Detrital zircon age patterns from turbidites of the Balagne and Piedmont nappes of Alpine Corsica (France): evidence for an European margin source. Tectonophysics 722, 69-105.

Linnemann, U., Gerdes, A., Drost, K., Buschmann, B., 2007. The continuum between Cadomian orogenesis and opening of the Rheic Ocean: Constraints from LA-ICP-MS U-Pb zircon dating and analysis of plate-tectonic setting (Saxo-Thuringian zone, NE Bohemian massif, Germany), in Linnemann, U., Nance, R.D., Kraft, P., and Zulauf, G., eds., The evolution of the Rheic Ocean: From Avalonian-Cadomian active margin to Alleghenian-Variscan collision: Geol. Soc. of Am. Spec. Paper 423, pp. 61-96.

Linnemann, U., McNaughton, N.J., Romer, R.L., Gehmlich, M., Drost, K., Tonk, C., 2004. West African provenance for Saxo-Thuringian (Bohemian Massif): did Armorica ever leave pre-Pangean Gondwana? U/Pb-SHRIMP zircon evidence and the Nd-isotopic record. Int. J. Earth Sci. 93, 683-705.

Marchand, J., 1981. Ecaillage d'un "mélange tectonique” profond: le complexe cristallophyllien de Champtoceaux (Bretagne Méridionale). C. R. Acad. Sci. Paris 293, 223-228

Martínez Catalán, J.R., Arenas, R., Abati, J., Sánchez Martínez, S., Díaz García, F., Fernández Suárez, J., González Cuadra, P., Castiñeiras, P., Gómez Barreiro, J., Díez Montes, A., González Clavijo, E., Rubio Pascual, F.J., Andonaegui, P., Jeffries, T.E., Alcock, J.E., Díez Fernández, R., López Carmona, A., 2009. A 
rootless suture and the loss of the roots of a mountain chain: the Variscan belt of NW Iberia. C.R. Géosci. 341, 114-126.

Martínez Catalán, J.R., Fernández-Suárez, J., Meireles, C., González-Clavijo, E., Belousova, E., Saeed, A., 2008. U-Pb detrital zircon ages in synorogenic deposits of the NW Iberian Massif (Variscan belt): interplay of Devonian-Carboniferous sedimentation and thrust tectonics. Int. J. Earth Sci. 165, 687-698.

Martínez Catalán, J.R., Fernández-Suárez, J., Jenner, G.A., Belousova, E., Díez Montes, A., 2004. Provenance constraints from detrital zircon $\mathrm{U}-\mathrm{Pb}$ ages in the NW Iberian Massif: implications from Palaeozoic plate configuration and Variscan evolution. J. Geol. Soc. London 161, 463-476.

Matte, P., 2001. The Variscan collage and orogeny (480-290 Ma) and the tectonic definition of the Armorica microplate: a review. Terra Nova 13, 122-128.

Melleton, J., Cocherie, A., Faure, M., Rossi, P., 2010. Precambrian protoliths and Early Paleozoic magmatism in the French Massif Central: U-Pb data and the North Gondwana connection in the west European Variscan belt. Gond. Res. 17, 13-25.

Morel, M.L., Nebel, A.O., Nebel-Jacobsen, Y.J., Miller, J.S., Vroon, P.Z., 2008. Hafnium isotope characterization of the GJ-1 zircon reference material by solution and laser-ablation MC-ICPMS. Chem. Geol. 255, 231-235.

Murphy, J.B., Gutierrez-Alonso, G., Nance, R.D., Fernandez-Suarez, J., Keppie, J.D., Quesada, C., Strachan, R.A., Dostal, J., 2006. Origin of the Rheic Ocean: Rifting along a Neoproterozoic suture? Geology 34, 325-328.

Murphy, J.B., Cousens, B.L., Braid, J.A., Strachan, R.A., Dostal, J., Keppie, J.D., Nance, R.D., 2011. Highly depleted oceanic lithosphere in the Rheic Ocean: implications for Paleozoic reconstructions. Lithos $123,165-175$

Murphy, J.B., Waldron, J.W.F., Schofield, D.I., Barry, T.L., Band, A.R., 2014. Highly depleted isotopic compositions evident in Iapetus and Rheic Ocean basalts: implications for crustal generation and preservation. Int. J. Earth Sci. (Geol. Rundsch.) 103, 1219-1232.

Paquette, J.L., Marchand, J., Peucat, J.J., 1984. Absence de tectonique cadomienne dans le complexe de Champtoceaux (Bretagne méridionale)? Comparaison des systèmes Rb-Sr et U-Pb d'un métagranite. Bull. de la Soc. Géol. de Fr. 26, 907-912.

Paquette J.L., Ballèvre M., Peucat J.J., Cornen G., 2017. From opening to subduction of an oceanic domain constrained by LA-ICP-MS U-Pb zircon dating (Variscan belt, southern Armorican Massif, France). Lithos 294-295, 418-437.

Pasteels, P., and Doré, F., 1982. Age of the Vire-Carolles granite. In: Odin, G.S. (Ed), Numerical dating in stratigraphy. pp. 784-790.

Peucat, J.J., 1986. Behaviour of Rb-Sr whole rock and U-Pb zircon systems during partial melting as shown in migmatitic gneisses from the Saint-Malo Massif, NE Brittany, France. J. Geol. Soc. London 143, 
$875-886$.

Pin, C., 1990. Variscan oceans: Ages, origins and geodynamic implications inferred from geochemical and radiometric data. Tectonophysics 177, 215-227.

Rolet, J., Gresselin, F., Jégouzo, P., Ledru, P., Wyns, R., 1994. Intracontinental Hercynian events in the Armorican Massif. In: Keppie J.D. (ed) Pre-Mezozoic geology of France and related areas Springer, Berlin, pp. 195-219.

Ruffet, G., Féraud, G., Amouric, M., 1991. Comparison of ${ }^{40} \mathrm{Ar}-{ }^{39} \mathrm{Ar}$ conventional and laser dating of biotites from the North Trégor Batholith. Géochimi. et Cosmochimi. Acta 55, 1675-1688.

Ruffet, G., Perroud, H., Féraud, G., 1990. ${ }^{40} \mathrm{Ar}-{ }^{39} \mathrm{Ar}$ dating of a late Proterozoic paleomagnetic pole for the Armorican Massif (France). Geophysical Journal International 102, 397-409.

Thiéblemont, D., Guerrot, C., Le Métour, J., Jézéquel, P., 2001. Le complexe de Cholet-Thouars: un ensemble volcano-plutonique cambrien moyen au sein du bloc précambrien des Mauges. Géol. de la Fr. 1-2, $3-17$.

van Achterbergh, E., Ryan, C., Jackson, S., Griffin, W.L., 2001. Appendix 3 data reduction software for LA-ICP-MS, in: Sylvester, P. (Eds.), Laser-Ablation-ICPMS in the Earth Sciences. Mineralogical Association of Canada, Short Courses 29, pp. 239-243.

Vermeesch, P., 2012. On the visualisation of detrital age distributions. Chem. Geol. 312-313, 190-194.

Vidal, P. 1980. L'évolution polyorogénique de Massif Armoricain : apport de la géochronologie et de la géochimie isotopique du strontium. Mémoire Soc. Géol. Minéral Bretagne, 21, Rennes, pp 1-162.

Winchester, J.A., Pharaoh, T.C., Verniers, J., 2002. Paleozoic Amalgamation of Central Europe: an introduction and synthesis of new results from recent geological and geophysical investigations. In: Winchester, J.A., Pharaoh, T.C., Verniers, J. (Eds.), Paleozoic Amalgamation of Central Europe Geol. Soc. of London, Spec. Publ, 201, pp. 1-18.

Woodhead, J.D., Hergt, J.M., 2005. A preliminary appraisal of seven natural zircon reference materials for in situ Hf isotope determination. Geostand. Geoanal. Res. 29, 183-195.

Wu, F.Y., Yang, Y.H., Xie, L.W., Yang, J.H., Xu, P., 2006. Hf isotopic compositions of the standard zircons and baddeleyites used in U-Pb geochronology. Chem. Geol. 234, 105-126.

Xie, L.W., Zhang, Y.B., Zhang, H.H., Sun, J.F., Wu, F.Y., 2008. In situ simultaneous determination of trace elements, U-Pb and Lu-Hf isotopes in zircon and baddeleyite. Chin. Sci. Bull. 53, 1565-1573.

\section{Figure captions}

Fig. 1. Tectonic map of the Armorican Massif (modified from Faure et al., 2005).

A: Location of the $\mathrm{S}^{\mathrm{t}}$-Georges-sur-Loire Unit and suture zones in the French Variscan belt. 
Fig. 2. Structural map of the Ligerian domain with sample locations. (SG: $\mathrm{S}^{\mathrm{t}}$-Georges-surLoire).

Fig. 3. Schematic cross section of the Ligerian domain (located in Fig. 2). Diamond: eclogites. Star: migmatitic leucosome with monazite U-Th-Pb chemical age at $387 \pm 6 \mathrm{Ma}$ (from Faure et al., 2008).

Fig. 4. Field photos and photomicrographs of the olistostrome sub-unit of the $\mathrm{S}^{\mathrm{t}}$-Georges-surLoire Unit.

A: Highly mature quartz-sandstone (Champtocé, 13FR12)

B: Lenticular coarse sandstone in a well-cleaved siltstone matrix of the olistostrome of $\mathrm{S}^{\mathrm{t}}$ Georges-sur-Loire (Villemoissan, sample 13FR18-1);

C: Chert enclosed in a cleaved siltstone matrix (Roche Airault, sample 13FR19);

D: Limestone and volcanic pebbles in a well-cleaved coarse sandstone conglomerate (Pierre Bize, sample 13FR21)

E: Well-bedded siltstone (Rochefort-sur-Loire, sample RO2);

F: Silty matrix with bedding $\left(S_{0}\right)$ and cleavage $\left(S_{1}\right)$ intersection in a normal limb of a Nverging fold (Villemoissan);

G: Elongated radiolarian with pressure shadows in a chert olistolith, Millé, location E in Fig. 2 ;

H: Volcaniclastic chlorite schist with shear bands, Faye d'Anjou, location F in Fig. 2.

Fig. 5. Photomicrographs of thin sections of dated rocks: A: Sandstone with detrital muscovite (ms) and plagioclase (pl) in sample 13FR12, Champtocé; B: Volcanic clasts in a 
rhyolitic block in sandstone, sample 13FR13, Epiré; C: coarse-grained sandstone of sample 13FR18-1, Villemoissan; D: Well-developed cleavage in muddy sandstone, sample 13FR19, Roche Airault; E: Polymictic sandstone with calcite (cal) cement, sample 13FR21, Pierre Bize; F: Muddy siltstone with detrital muscovite (ms) and plagioclase (pl), sample RO2, Rochefort-sur-Loire; G: Coarse-grained sandstone with detrital muscovite, sample OUT, $\mathrm{N}$ of St Georges; H: sandstone with deformed quartz grain with pressure shadows, and detrital zircon (zr), sample MB2, Moulin de Beaupréau.

Fig. 6. Representative cathodoluminescence (CL) images of selected detrital zircons from the olistostrome sub-unit of the $\mathrm{S}^{\mathrm{t}}$-Georges-sur-Loire. The solid and dashed circles represent U$\mathrm{Pb}$ and $\mathrm{Lu}-\mathrm{Hf}$ isotopic analytical sites, respectively. Analytical results are presented in Tables 2 and 3.

Fig. 7. Probability diagrams of U-Pb ages of the detrital zircons from the olistostrome subunit of the $S^{t}$-Georges-sur-Loire. See Fig. 2 for sample locations, and Table 2 for zircon ages.

Fig. 8. Diagrams of $\varepsilon_{\mathrm{Hf}}(\mathrm{t})$ values from the same samples as those analyzed for detrital zircons in the $\mathrm{S}^{\mathrm{t}}$-Georges-sur-Loire olistostrome unit.

Fig. 9. Diagrams of $\mathrm{T}_{\mathrm{DM}}{ }^{2}$ values from the same samples as those analyzed for detrital zircons in the St-Georges-sur-Loire olistostrome unit.

Fig. 10. A: Diagram of $\varepsilon_{\mathrm{Hf}}(\mathrm{t})$. B: Diagram of the two-stage model $\mathrm{Hf}$ age $\left(\mathrm{T}_{\mathrm{DM}}{ }^{2}\right)$ versus age plots for detrital zircons, manifesting the source of zircons. Zircon Hf model ages are older 
than their crystallization ages, revealing a formation from re-melting of older crustal rocks crystallized during the previous events.

Fig. 11. A. Synthesis and comparison of cumulative probability plots of detrital zircon U-Pb ages from the olistostrome sub-unit of St-Georges-sur-Loire; B. Ancenis Basin, and Late Carboniferous coal-bearing basin of Basse Loire (from Ducassou et al., 2014). Histograms only include concordant detrital zircon ages obtained in this study.

Fig. 12. (A) Temporal variations of $\varepsilon_{\mathrm{Hf}}(\mathrm{t})$ values at $0-4.0 \mathrm{Ga}$ of this study. The crustal evolution range comes from Linnemann et al. (2014). Dashed lines demonstrate evolution of zircons with depleted mantle model ages between $1.2 \mathrm{Ga}, 1.8 \mathrm{Ga}$ and $2.8 \mathrm{Ga}$. Half of the zircons plots in the crustal evolution zone between $1.2 \mathrm{Ga}$ and $1.8 \mathrm{Ga}$, but Archean data are consistent with the range of the Archean crust. (B) Diagram of $\varepsilon_{\mathrm{Hf}}(\mathrm{t})$ value versus age for younger zircons at 800-400 Ma.

Fig. 13. Synthetic and comparison of the cumulative probability plots of detrital zircon U-Pb ages from the olistostrome sub-unit of the $\mathrm{S}^{\mathrm{t}}$-Georges-sur-Loire Unit (this study), Ancenis Basin and Coal-bearing basin of the Basse-Loire (Ducassou et al., 2014), Late Paleozoic sedimentary sequences of the recumbent folds and the foreland basin of the Montagne Noire (Lin et al., 2016), Cadomian metasediments and Ordovician sandstone of Corsica (Avigad et al., 2018), and West Avalonia (Henderson et al., 2016, 2018).

Fig. 14. Schematic model of the Silurian-Early Devonian (ca. 440-400 Ma) oceanic convergence between Armorica and Gondwana that led to the closure of the Medio-European Ocean (modified from Faure et al., 2008). The southern continental margin of Armorica is a 
magmatic arc-back arc system. Detrital zircon analyses from the $\mathrm{S}^{\mathrm{t}}$-Georges-sur-Loire Unit indicate Early Paleozoic, Neoproterozoic and Paleoproterozoic-Archean sources. Presently, the Icartian basement, Cadomian orogenic belt and Early Paleozoic magmatic rocks are exposed in North-and Central Armorica and to a lesser extent, in the basement of the magmatic arc now only preserved in the Mauges nappe.

Table 1: Summary of the analyzed samples from the olistostrome of the sub-unit of the $\mathrm{S}^{\mathrm{t}}$-Georges-sur-Loire Unit.

Table 2: Detrital Zircon U-Pb analytical data. Discordance (\%) is calculated as (1) $100 \% *\left({ }^{207} \mathrm{~Pb} /{ }^{206} \mathrm{~Pb}\right.$ age $-{ }^{206} \mathrm{~Pb} /{ }^{238} \mathrm{U}$ age $) /\left({ }^{207} \mathrm{~Pb} /{ }^{206} \mathrm{~Pb}\right.$ age $)$, if the ${ }^{206} \mathrm{~Pb} /{ }^{238} \mathrm{U}$ age is older than $1000 \mathrm{Ma}$; (2) $100 \% *\left({ }^{207} \mathrm{~Pb} /{ }^{235} \mathrm{U}\right.$ age $-{ }^{206} \mathrm{~Pb} /{ }^{238} \mathrm{U}$ age $) /\left({ }^{206} \mathrm{~Pb} /{ }^{238} \mathrm{U}\right.$ age $)$, if the ${ }^{206} \mathrm{~Pb} /{ }^{238} \mathrm{U}$ age is younger than $1000 \mathrm{Ma}$.

Table 3: LA-ICP-MS zircon Lu-Hf isotopic data. Age is the same from Table 2 $\mathrm{T}(\mathrm{DM})$ and $\mathrm{T}(\mathrm{DM} 2)$ are one-stage and two-stage Hf model ages, respectively. ${ }^{176} \mathrm{Hf} /{ }^{177} \mathrm{Hf}(\mathrm{CHUR}, \mathrm{t})=0.282772-0.0332 *(\mathrm{EXP}(0.01867 *$ Age $/ 1000)-1) .{ }^{176} \mathrm{Hf} /{ }^{177} \mathrm{Hf}$ $(\mathrm{DM}, \mathrm{t})=0.28325-0.0384 *(\mathrm{EXP}(0.01867 * \mathrm{Age} / 1000)-1) . \mathrm{T}_{\mathrm{DM} 1}=(1 / 0.01867) * \mathrm{LN}(1+$ $\left.\left(\left({ }^{176} \mathrm{Hf} /{ }^{177} \mathrm{Hf}-0.28325\right) /\left({ }^{176} \mathrm{Lu} /{ }^{177} \mathrm{Hf}-0.0384\right)\right)\right) . \mathrm{T}_{\mathrm{DM} 2}=$ Age $/ 1000+(1 / 0.01867) * \mathrm{LN}(1+$ $\left.\left({ }^{176} \mathrm{Hf} /{ }^{177} \mathrm{Hf}(\sigma, \mathrm{t})-{ }^{176} \mathrm{Hf} /{ }^{177} \mathrm{Hf}(\mathrm{DM}, \mathrm{t})\right) /(0.015-0.0384)\right)$. 


\section{Table 1}

Stratigraphic Age Sample ${ }^{\circ}$ GPS location Petrography Tectonic position

\begin{tabular}{|c|c|c|c|}
\hline 13FR12 & $\begin{array}{l}47^{\circ} 24^{\prime} 45.44^{\prime \prime} \mathrm{N} \\
00^{\circ} 51^{\prime} 37.60^{\prime \prime} \mathrm{W} \\
\end{array}$ & $\begin{array}{l}\text { Sandstone } \\
\text { (matrix) }\end{array}$ & $\begin{array}{l}\text { Olistostrome sub-unit of } \\
\text { the St-Georges-sur-Loire }\end{array}$ \\
\hline 13FR13 & $\begin{array}{l}47^{\circ} 23^{\prime} 43.71 " \mathrm{~N} \\
00^{\circ} 38^{\prime} 39.38^{\prime \prime} \mathrm{W} \\
\end{array}$ & $\begin{array}{l}\text { Rhyolite block in } \\
\text { siltstone matrix }\end{array}$ & $\begin{array}{l}\text { Rhyolite blocks in the olistostrome } \\
\text { sub-unit of the St-Georges-sur-Loi }\end{array}$ \\
\hline 13FR18-1 & $\begin{array}{l}47^{\circ} 27^{\prime} 40.95^{\prime \prime} \mathrm{N} \\
00^{\circ} 53^{\prime} 18.69^{\prime \prime} \mathrm{W} \\
\end{array}$ & $\begin{array}{l}\text { Lenticular coarse sandsto } \\
\text { in siltstone matrix }\end{array}$ & $\begin{array}{l}\text { Olistostrome sub-unit of } \\
\text { the St-Georges-sur-Loire }\end{array}$ \\
\hline 13FR19 & $\begin{array}{l}47^{\circ} 20^{\prime} 47.81^{\prime \prime} \mathrm{N} \\
00^{\circ} 40^{\prime} 48.54^{\prime \prime} \mathrm{W}\end{array}$ & Muddy sandstone matrix & $\begin{array}{l}\text { Olistostrome sub-unit of } \\
\text { the St-Georges-sur-Loire }\end{array}$ \\
\hline 13 FR 21 & $\begin{array}{l}47^{\circ} 28^{\prime} 56.51 " \mathrm{~N} \\
00^{\circ} 36^{\prime} 51.39^{\prime \prime} \mathrm{W} \\
\end{array}$ & Sandstone matrix & $\begin{array}{l}\text { Olistostrome sub-unit of } \\
\text { the St-Georges-sur-Loire }\end{array}$ \\
\hline $\mathrm{RO} 2$ & $\begin{array}{l}47^{\circ} 20^{\prime} 54.02^{\prime \prime} \mathrm{N} \\
0^{\circ} 38^{\prime} 3.33^{\prime \prime} \mathrm{W} \\
\end{array}$ & Sandstone & $\begin{array}{l}\text { Olistostrome sub-unit of } \\
\text { the St-Georges-sur-Loire }\end{array}$ \\
\hline OUT & $\begin{array}{l}47^{\circ} 26^{\prime} 09.17^{\prime \prime} \mathrm{N} \\
0^{\circ} 45^{\prime} 25.15^{\prime \prime} \mathrm{W} \\
\end{array}$ & Sandstone & $\begin{array}{l}\text { Olistostrome sub-unit of } \\
\text { the St-Georges-sur-Loire }\end{array}$ \\
\hline MB2 & $\begin{array}{l}47^{\circ} 24^{\prime} 3.04^{\prime \prime} \mathrm{N} \\
0^{\circ} 39^{\prime} 13.74^{\prime \prime} \mathrm{W}\end{array}$ & Sandstone & $\begin{array}{l}\text { Olistostrome sub-unit of } \\
\text { the St-Georges-sur-Loire }\end{array}$ \\
\hline
\end{tabular}


Table 2. LA-ICPMA Zircon U-Pb analytical data

\begin{tabular}{|c|c|c|c|c|c|c|c|c|c|c|c|c|c|c|c|c|}
\hline & & & & & & & & & & \multirow{2}{*}{\multicolumn{6}{|c|}{ Ages (Ma) }} & \multirow[b]{3}{*}{$\begin{array}{c}\text { Disconcorda } \\
\text { nce }\end{array}$} \\
\hline \multirow[b]{2}{*}{$\begin{array}{l}\text { Sample\#sp } \\
\text { ot }\end{array}$} & \multirow[b]{2}{*}{$\begin{array}{c}\mathrm{Th} \\
(\mathrm{ppm})\end{array}$} & \multirow[b]{2}{*}{$\begin{array}{c}\mathrm{U} \\
(\mathrm{ppm})\end{array}$} & \multirow[b]{2}{*}{$\begin{array}{c}\mathrm{Th} / \\
\mathrm{U}\end{array}$} & \multicolumn{6}{|c|}{ Isotopic ratios } & & & & & & & \\
\hline & & & & $\begin{array}{c}{ }^{207} \mathrm{~Pb} /{ }^{206} \\
\mathrm{~Pb}\end{array}$ & $\pm 1 \sigma$ & $\begin{array}{c}{ }^{207} \mathrm{~Pb} /{ }^{23} \\
{ }^{5} \mathrm{U}\end{array}$ & $\pm 1 \sigma$ & $\begin{array}{c}{ }^{206} \mathrm{~Pb} /{ }^{23} \\
{ }^{8} \mathrm{U}\end{array}$ & $\pm 1 \sigma$ & $\begin{array}{c}{ }^{207} \mathrm{~Pb} /{ }^{206} \\
\mathrm{~Pb}\end{array}$ & $\begin{array}{c} \pm \\
1 \sigma\end{array}$ & $\begin{array}{c}{ }^{207} \mathrm{~Pb} /{ }^{23} \\
{ }^{5} \mathrm{U}\end{array}$ & $\begin{array}{c} \pm \\
1 \sigma\end{array}$ & $\begin{array}{c}{ }^{206} \mathrm{~Pb} /{ }^{23} \\
{ }^{8} \mathrm{U}\end{array}$ & $\begin{array}{l} \pm \\
1 \\
\sigma\end{array}$ & \\
\hline
\end{tabular}

\section{FR12}

\begin{tabular}{|c|c|c|c|c|c|c|c|c|c|c|c|c|c|c|c|c|}
\hline 13FR12\#01 & 148 & 196 & $\begin{array}{c}0.7 \\
6\end{array}$ & 0.12772 & $\begin{array}{c}0.002 \\
45\end{array}$ & $\begin{array}{c}6.3155 \\
0\end{array}$ & $\begin{array}{c}0.115 \\
45\end{array}$ & $\begin{array}{c}0.3588 \\
0\end{array}$ & $\begin{array}{c}0.005 \\
27\end{array}$ & 206 & & 2021 & 16 & 1976 & 2 & $4.61 \%$ \\
\hline 13FR12\#02 & 211 & 440 & $\begin{array}{c}0.4 \\
8\end{array}$ & 0.05647 & $\begin{array}{c}0.003 \\
03\end{array}$ & $\begin{array}{c}0.6607 \\
3\end{array}$ & $\begin{array}{c}0.033 \\
89\end{array}$ & $\begin{array}{c}0.0849 \\
0\end{array}$ & $\begin{array}{c}0.001 \\
78\end{array}$ & & 76 & 515 & 21 & 525 & 1 & $-1.90 \%$ \\
\hline 3FR12\#03 & 250 & 285 & $\begin{array}{c}0.8 \\
8\end{array}$ & 0.05868 & $\begin{array}{c}0.002 \\
60\end{array}$ & $\begin{array}{c}0.8283 \\
1\end{array}$ & $\begin{array}{c}0.035 \\
07\end{array}$ & $\begin{array}{c}0.1024 \\
3\end{array}$ & $\begin{array}{c}0.001 \\
90\end{array}$ & 555 & 60 & 613 & 19 & 629 & $\begin{array}{l}1 \\
1\end{array}$ & $-2.54 \%$ \\
\hline 3FR12\#04 & 138 & 795 & $\begin{array}{c}0.1 \\
7\end{array}$ & 0.17388 & $\begin{array}{c}0.001 \\
84\end{array}$ & $\begin{array}{c}12.107 \\
62\end{array}$ & $\begin{array}{c}0.124 \\
94\end{array}$ & $\begin{array}{c}0.5052 \\
7\end{array}$ & $\begin{array}{c}0.006 \\
12\end{array}$ & 2595 & 9 & 2613 & 10 & 2636 & $\begin{array}{l}2 \\
6\end{array}$ & $-1.56 \%$ \\
\hline 3FR12\#05 & 210 & 216 & $\begin{array}{c}0.9 \\
7\end{array}$ & 0.06366 & $\begin{array}{c}0.003 \\
50\end{array}$ & $\begin{array}{c}0.9815 \\
4\end{array}$ & $\begin{array}{c}0.051 \\
42\end{array}$ & $\begin{array}{c}0.1118 \\
8\end{array}$ & $\begin{array}{c}0.002 \\
51\end{array}$ & 730 & 73 & 694 & 26 & 684 & $\begin{array}{l}1 \\
5\end{array}$ & $1.46 \%$ \\
\hline 3FR12\#06 & 172 & 236 & $\begin{array}{c}0.7 \\
3\end{array}$ & 0.06973 & $\begin{array}{c}0.003 \\
51\end{array}$ & 1.0561 & $\begin{array}{l}0.050 \\
36\end{array}$ & $\begin{array}{c}0.1098 \\
9\end{array}$ & $\begin{array}{c}0.002 \\
39\end{array}$ & 920 & 62 & 732 & 25 & 672 & $\begin{array}{l}1 \\
4\end{array}$ & $8.93 \%$ \\
\hline 13FR12\#07 & 54 & 271 & $\begin{array}{c}0.2 \\
0\end{array}$ & 0.06969 & $\begin{array}{c}0.002 \\
24\end{array}$ & $\begin{array}{c}1.5418 \\
4\end{array}$ & $\begin{array}{c}0.047 \\
14\end{array}$ & $\begin{array}{c}0.1605 \\
2\end{array}$ & $\begin{array}{c}0.002 \\
60\end{array}$ & 919 & 27 & 947 & 19 & 960 & 4 & $-1.35 \%$ \\
\hline 13FR12\#08 & 144 & 297 & $\begin{array}{c}0.4 \\
9\end{array}$ & 0.1362 & $\begin{array}{c}0.002 \\
27\end{array}$ & $\begin{array}{c}7.7512 \\
1\end{array}$ & $\begin{array}{c}0.123 \\
98\end{array}$ & $\begin{array}{c}0.4126 \\
9\end{array}$ & $\begin{array}{c}0.005 \\
77\end{array}$ & 2180 & 12 & 2203 & 14 & 2227 & $\begin{array}{l}2 \\
6\end{array}$ & $-2.11 \%$ \\
\hline 13FR12\#09 & 157 & 187 & $\begin{array}{c}0.8 \\
4\end{array}$ & & $\begin{array}{c}0.00 \\
40\end{array}$ & $\begin{array}{c}0.8337 \\
7\end{array}$ & $\begin{array}{c}0.045 \\
08\end{array}$ & $\begin{array}{c}0.1009 \\
6\end{array}$ & $\begin{array}{c}0.002 \\
25\end{array}$ & 601 & 78 & 616 & 25 & 620 & $\begin{array}{l}1 \\
3\end{array}$ & $-0.65 \%$ \\
\hline 13FR12\#10 & 79 & 177 & $\begin{array}{c}0.4 \\
5\end{array}$ & 0.12964 & $\begin{array}{c}0.002 \\
58\end{array}$ & $\begin{array}{c}7.2340 \\
1\end{array}$ & $\begin{array}{c}0.138 \\
39\end{array}$ & $\begin{array}{c}0.4048 \\
8\end{array}$ & $\begin{array}{c}0.006 \\
16\end{array}$ & 2093 & 15 & 2141 & 17 & 2191 & $\begin{array}{l}2 \\
8\end{array}$ & $-4.47 \%$ \\
\hline 13FR12\#11 & 144 & 167 & $\begin{array}{c}0.8 \\
6\end{array}$ & 0.11373 & $\begin{array}{c}0.002 \\
85\end{array}$ & $\begin{array}{c}5.2902 \\
3\end{array}$ & $\begin{array}{c}0.126 \\
20\end{array}$ & $\begin{array}{c}0.3375 \\
0\end{array}$ & $\begin{array}{c}0.005 \\
62\end{array}$ & 1860 & 21 & 1867 & 20 & 1875 & $\begin{array}{l}2 \\
7\end{array}$ & $-0.80 \%$ \\
\hline 13FR12\#12 & 111 & 232 & $\begin{array}{c}0.4 \\
8\end{array}$ & 0.11458 & $\begin{array}{c}0.002 \\
33\end{array}$ & $\begin{array}{c}5.3299 \\
8\end{array}$ & $\begin{array}{c}0.103 \\
28\end{array}$ & $\begin{array}{c}0.3375 \\
0\end{array}$ & $\begin{array}{c}0.004 \\
98\end{array}$ & 1873 & 16 & 1874 & 17 & 1875 & $\begin{array}{l}2 \\
4\end{array}$ & $-0.11 \%$ \\
\hline 13FR12\#13 & 80 & 102 & $\begin{array}{c}0.7 \\
8\end{array}$ & 0.11319 & $\begin{array}{c}0.003 \\
47\end{array}$ & $\begin{array}{c}5.4972 \\
7\end{array}$ & $\begin{array}{c}0.161 \\
00\end{array}$ & $\begin{array}{c}0.3523 \\
7\end{array}$ & $\begin{array}{c}0.006 \\
77\end{array}$ & 1851 & 27 & 1900 & 25 & 1946 & $\begin{array}{l}3 \\
2\end{array}$ & $-4.88 \%$ \\
\hline 3FR12\#14 & 102 & 108 & $\begin{array}{c}0.9 \\
4\end{array}$ & 0.11413 & $\begin{array}{c}0.003 \\
28\end{array}$ & $\begin{array}{c}5.0950 \\
2\end{array}$ & $\begin{array}{c}0.139 \\
04\end{array}$ & $\begin{array}{c}0.3239 \\
1\end{array}$ & $\begin{array}{c}0.005 \\
88\end{array}$ & 1866 & 25 & 1835 & 23 & 1809 & $\begin{array}{l}2 \\
9\end{array}$ & $3.15 \%$ \\
\hline
\end{tabular}




\begin{tabular}{|c|c|c|c|c|c|c|c|c|c|c|c|c|c|c|c|c|}
\hline 13FR12\#15 & 51 & 83 & $\begin{array}{c}0.6 \\
1\end{array}$ & 0.11423 & $\begin{array}{c}0.003 \\
93\end{array}$ & $\begin{array}{c}5.3303 \\
8\end{array}$ & $\begin{array}{c}0.174 \\
47\end{array}$ & $\begin{array}{c}0.3385 \\
7\end{array}$ & $\begin{array}{c}0.007 \\
05\end{array}$ & 1868 & 31 & 1874 & 28 & 1880 & 3 & $-0.64 \%$ \\
\hline 13FR12\#16 & 388 & 400 & $\begin{array}{c}0.9 \\
7\end{array}$ & 0.11323 & $\begin{array}{c}0.001 \\
89\end{array}$ & $\begin{array}{c}5.2054 \\
4\end{array}$ & $\begin{array}{c}0.083 \\
11\end{array}$ & $\begin{array}{c}0.3335 \\
4\end{array}$ & $\begin{array}{c}0.004 \\
46\end{array}$ & 1852 & 13 & 1854 & 14 & 1856 & ? & $-0.22 \%$ \\
\hline 13FR12\#17 & 253 & 373 & $\begin{array}{c}0.6 \\
8\end{array}$ & 0.05723 & $\begin{array}{c}0.002 \\
45\end{array}$ & $\begin{array}{c}0.7319 \\
4\end{array}$ & $\begin{array}{c}0.029 \\
98\end{array}$ & $\begin{array}{c}0.0927 \\
9\end{array}$ & $\begin{array}{c}0.001 \\
66\end{array}$ & 500 & 59 & 558 & 18 & 572 & $\begin{array}{l}1 \\
0\end{array}$ & $-2.45 \%$ \\
\hline 13FR12\#18 & 154 & 201 & $\begin{array}{c}0.7 \\
7\end{array}$ & 0.05940 & $\begin{array}{c}0.002 \\
87\end{array}$ & $\begin{array}{c}0.7881 \\
1\end{array}$ & $\begin{array}{c}0.036 \\
34\end{array}$ & $\begin{array}{c}0.0962 \\
6\end{array}$ & $\begin{array}{c}0.001 \\
90\end{array}$ & 582 & & & 21 & 592 & 1 & $-0.34 \%$ \\
\hline 13FR12\#19 & 298 & 549 & $\begin{array}{c}0.5 \\
4\end{array}$ & 0.05815 & $\begin{array}{c}0.001 \\
84\end{array}$ & $\begin{array}{c}0.7044 \\
6\end{array}$ & $\begin{array}{c}0.021 \\
32\end{array}$ & $\begin{array}{c}0.0879 \\
0\end{array}$ & $\begin{array}{c}0.001 \\
32\end{array}$ & & & & 13 & 543 & 8 & $-0.37 \%$ \\
\hline 13FR12\#20 & 106 & 202 & $\begin{array}{c}0.5 \\
2\end{array}$ & 0.18067 & $\begin{array}{c}0.002 \\
55\end{array}$ & $\begin{array}{c}12.946 \\
09\end{array}$ & $\begin{array}{c}0.178 \\
06\end{array}$ & $\begin{array}{c}0.5198 \\
7\end{array}$ & $\begin{array}{c}0.007 \\
17\end{array}$ & 2659 & 10 & 2676 & 13 & 2699 & $\begin{array}{l}3 \\
0\end{array}$ & $-1.48 \%$ \\
\hline 13FR12\#21 & 69 & 52 & $\begin{array}{c}1.3 \\
3\end{array}$ & 0.06497 & $\begin{array}{c}0.005 \\
92\end{array}$ & $\begin{array}{c}0.8894 \\
0\end{array}$ & $\begin{array}{c}0.076 \\
98\end{array}$ & $\begin{array}{c}0.0993 \\
1\end{array}$ & $\begin{array}{c}0.003 \\
44\end{array}$ & 773 & $\begin{array}{c}12 \\
4\end{array}$ & 646 & 41 & 610 & 2 & $5.90 \%$ \\
\hline 13FR12\#22 & 151 & 80 & $\begin{array}{l}1.8 \\
7\end{array}$ & 0.05756 & $\begin{array}{c}0.004 \\
66\end{array}$ & $\begin{array}{c}0.8236 \\
0\end{array}$ & $\begin{array}{c}0.064 \\
06\end{array}$ & $\begin{array}{c}0.1038 \\
0\end{array}$ & $\begin{array}{c}0.002 \\
94\end{array}$ & 513 & $\begin{array}{c}12 \\
1\end{array}$ & 610 & 36 & 637 & 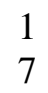 & $-4.24 \%$ \\
\hline 13FR12\#23 & 60 & 86 & $\begin{array}{c}0.6 \\
9\end{array}$ & 0.06338 & $\begin{array}{c}0.004 \\
99\end{array}$ & $\begin{array}{c}0.7903 \\
9\end{array}$ & $\begin{array}{c}0.059 \\
22\end{array}$ & $\begin{array}{c}0.0904 \\
7\end{array}$ & $\begin{array}{c}0.002 \\
68\end{array}$ & 721 & $\begin{array}{c}10 \\
9\end{array}$ & 591 & 34 & 558 & 6 & $5.91 \%$ \\
\hline 13FR12\#24 & 240 & 371 & $\begin{array}{c}0.6 \\
5\end{array}$ & 0.06232 & $\begin{array}{c}0.002 \\
92\end{array}$ & 0.8907 & $\begin{array}{c}0.039 \\
68\end{array}$ & $\begin{array}{c}0.1037 \\
0\end{array}$ & $\begin{array}{c}0.002 \\
05\end{array}$ & 685 & 61 & 647 & 21 & 636 & $\begin{array}{l}1 \\
2\end{array}$ & $1.73 \%$ \\
\hline 13FR12\#25 & 148 & 100 & $\begin{array}{c}1.4 \\
7\end{array}$ & 0.12563 & $\begin{array}{c}0.003 \\
24\end{array}$ & $\begin{array}{c}6.4086 \\
8\end{array}$ & $\begin{array}{c}0.157 \\
39\end{array}$ & $\begin{array}{c}0.3700 \\
8\end{array}$ & $\begin{array}{c}0.006 \\
50\end{array}$ & 2038 & 21 & 2033 & 22 & 2030 & $\begin{array}{l}3 \\
1\end{array}$ & $0.39 \%$ \\
\hline 13FR12\#26 & 177 & 181 & $\begin{array}{c}0.9 \\
8\end{array}$ & 0.05986 & $\begin{array}{c}0.003 \\
04\end{array}$ & $\begin{array}{c}0.8066 \\
2\end{array}$ & $\begin{array}{c}0.039 \\
08\end{array}$ & $\begin{array}{c}0.0977 \\
6\end{array}$ & $\begin{array}{c}0.001 \\
98\end{array}$ & 599 & 70 & 601 & 22 & 601 & $\begin{array}{l}1 \\
2\end{array}$ & $0.00 \%$ \\
\hline 13FR12\#27 & 218 & 388 & $\begin{array}{c}0.5 \\
6\end{array}$ & 0.06369 & $\begin{array}{c}0.002 \\
29\end{array}$ & $\begin{array}{c}0.9121 \\
7\end{array}$ & $\begin{array}{c}0.031 \\
25\end{array}$ & $\begin{array}{c}0.1039 \\
0\end{array}$ & $\begin{array}{c}0.001 \\
72\end{array}$ & 731 & 45 & 658 & 17 & 637 & $\begin{array}{l}1 \\
0\end{array}$ & $3.30 \%$ \\
\hline 13FR12\#28 & 113 & 283 & $\begin{array}{c}0.4 \\
0\end{array}$ & 0.05884 & $\begin{array}{c}0.002 \\
77\end{array}$ & $\begin{array}{c}0.7660 \\
1\end{array}$ & $\begin{array}{c}0.034 \\
31\end{array}$ & $\begin{array}{c}0.0944 \\
5\end{array}$ & $\begin{array}{c}0.001 \\
83\end{array}$ & 561 & 64 & 577 & 20 & 582 & $\begin{array}{l}1 \\
1\end{array}$ & $-0.86 \%$ \\
\hline 13FR12\#29 & 109 & 43 & $\begin{array}{c}2.5 \\
2\end{array}$ & 0.11598 & $\begin{array}{c}0.005 \\
13\end{array}$ & $\begin{array}{c}5.8357 \\
6\end{array}$ & $\begin{array}{c}0.246 \\
84\end{array}$ & $\begin{array}{c}0.3650 \\
2\end{array}$ & $\begin{array}{c}0.009 \\
25\end{array}$ & 1895 & 41 & 1952 & 37 & 2006 & $\begin{array}{l}4 \\
4\end{array}$ & $-5.53 \%$ \\
\hline $13 F R 12 \# 30$ & 391 & 662 & $\begin{array}{c}0.5 \\
9\end{array}$ & 0.11630 & $\begin{array}{c}0.004 \\
21\end{array}$ & $\begin{array}{c}1.6568 \\
3\end{array}$ & $\begin{array}{c}0.054 \\
95\end{array}$ & $\begin{array}{c}0.1033 \\
5\end{array}$ & $\begin{array}{c}0.002 \\
04\end{array}$ & 1900 & 32 & 992 & 21 & 634 & $\begin{array}{l}1 \\
2\end{array}$ & $56.47 \%$ \\
\hline 13FR12\#31 & 161 & 182 & $\begin{array}{c}0.8 \\
9\end{array}$ & 0.12360 & $\begin{array}{c}0.002 \\
37\end{array}$ & $\begin{array}{c}6.4115 \\
4\end{array}$ & $\begin{array}{c}0.117 \\
54\end{array}$ & $\begin{array}{c}0.3763 \\
3\end{array}$ & $\begin{array}{c}0.005 \\
51\end{array}$ & 2009 & 15 & 2034 & 16 & 2059 & $\begin{array}{l}2 \\
6\end{array}$ & $-2.43 \%$ \\
\hline 13FR12\#32 & 132 & 257 & 0.5 & 0.05858 & 0.002 & 0.6405 & 0.028 & 0.0793 & 0.001 & 552 & 63 & 503 & 17 & 492 & 9 & $2.24 \%$ \\
\hline
\end{tabular}




\begin{tabular}{|c|c|c|c|c|c|c|c|c|c|c|c|c|c|c|c|c|}
\hline & & & 2 & & 71 & 0 & 21 & 2 & 50 & & & & & & & \\
\hline 13FR12\#33 & 72 & 81 & $\begin{array}{c}0.8 \\
9\end{array}$ & 0.05642 & $\begin{array}{c}0.004 \\
61\end{array}$ & $\begin{array}{c}0.7150 \\
0\end{array}$ & $\begin{array}{c}0.055 \\
98\end{array}$ & $\begin{array}{c}0.0919 \\
3\end{array}$ & $\begin{array}{c}0.002 \\
62\end{array}$ & 469 & $\begin{array}{c}12 \\
3\end{array}$ & 548 & 33 & 567 & $\begin{array}{l}1 \\
5\end{array}$ & $-3.35 \%$ \\
\hline 13FR12\#34 & 73 & 147 & $\begin{array}{c}0.5 \\
0\end{array}$ & 0.11415 & $\begin{array}{c}0.002 \\
63\end{array}$ & $\begin{array}{c}4.8728 \\
5\end{array}$ & $\begin{array}{c}0.106 \\
43\end{array}$ & $\begin{array}{c}0.3096 \\
8\end{array}$ & $\begin{array}{c}0.004 \\
86\end{array}$ & 1867 & 19 & 1798 & 18 & 1739 & $\begin{array}{l}2 \\
4\end{array}$ & $7.36 \%$ \\
\hline 13FR12\#35 & 79 & 114 & $\begin{array}{c}0.6 \\
9\end{array}$ & 0.26888 & $\begin{array}{c}0.003 \\
61\end{array}$ & $\begin{array}{c}24.517 \\
40\end{array}$ & $\begin{array}{c}0.327 \\
84\end{array}$ & $\begin{array}{c}0.6614 \\
6\end{array}$ & $\begin{array}{c}0.009 \\
70\end{array}$ & 3300 & 10 & 32 & 13 & 3273 & $\begin{array}{l}3 \\
8\end{array}$ & $0.82 \%$ \\
\hline $13 F R 12 \# 36$ & 321 & 522 & $\begin{array}{c}0.6 \\
1\end{array}$ & 0.06598 & $\begin{array}{c}0.002 \\
01\end{array}$ & $\begin{array}{c}0.7515 \\
7\end{array}$ & $\begin{array}{c}0.021 \\
72\end{array}$ & $\begin{array}{c}0.0826 \\
3\end{array}$ & $\begin{array}{c}0.001 \\
25\end{array}$ & 806 & & & 13 & 512 & 7 & $11.13 \%$ \\
\hline 13FR12\#37 & 77 & 242 & $\begin{array}{c}0.3 \\
2\end{array}$ & 0.12664 & $\begin{array}{c}0.002 \\
37\end{array}$ & $\begin{array}{c}6.3769 \\
7\end{array}$ & $\begin{array}{c}0.113 \\
83\end{array}$ & $\begin{array}{c}0.3652 \\
8\end{array}$ & $\begin{array}{c}0.005 \\
29\end{array}$ & 2052 & 14 & 2029 & 16 & 2007 & $\begin{array}{l}2 \\
5\end{array}$ & $2.24 \%$ \\
\hline 13FR12\#38 & 114 & 126 & $\begin{array}{c}0.9 \\
1\end{array}$ & 0.11283 & $\begin{array}{c}0.002 \\
65\end{array}$ & $\begin{array}{c}5.3551 \\
9\end{array}$ & $\begin{array}{c}0.119 \\
86\end{array}$ & $\begin{array}{c}0.3443 \\
1\end{array}$ & 0.005 & 1845 & 20 & 1878 & 19 & 1907 & $\begin{array}{l}2 \\
6\end{array}$ & $-3.25 \%$ \\
\hline 13FR12\#39 & 129 & 230 & $\begin{array}{c}0.5 \\
6\end{array}$ & 0.11583 & $\begin{array}{c}0.002 \\
23\end{array}$ & $\begin{array}{c}5.2898 \\
4\end{array}$ & $\begin{array}{c}0.096 \\
97\end{array}$ & $\begin{array}{c}0.3312 \\
8\end{array}$ & $\begin{array}{c}0.004 \\
75\end{array}$ & 1893 & 15 & 1867 & 16 & 1845 & $\begin{array}{l}2 \\
3\end{array}$ & $2.60 \%$ \\
\hline 13FR12\#40 & 26 & 53 & $\begin{array}{c}0.4 \\
9\end{array}$ & 0.12969 & $\begin{array}{c}0.004 \\
40\end{array}$ & $\begin{array}{c}6.7873 \\
1\end{array}$ & $\begin{array}{c}0.219 \\
53\end{array}$ & $\begin{array}{c}0.3796 \\
4\end{array}$ & $\begin{array}{c}0.008 \\
25\end{array}$ & 2094 & 29 & 2084 & 29 & 2075 & $\begin{array}{l}3 \\
9\end{array}$ & $0.92 \%$ \\
\hline 13FR12\#41 & 186 & 186 & $\begin{array}{c}1.0 \\
0\end{array}$ & 0.12854 & $\begin{array}{c}0.002 \\
16\end{array}$ & $\begin{array}{c}6.6779 \\
7\end{array}$ & $\begin{array}{c}0.107 \\
40\end{array}$ & $\begin{array}{c}0.3768 \\
6\end{array}$ & $\begin{array}{c}0.005 \\
20\end{array}$ & 2078 & 13 & 2070 & 14 & 2062 & $\begin{array}{l}2 \\
4\end{array}$ & $0.78 \%$ \\
\hline 13FR12\#42 & 188 & 231 & $\begin{array}{c}0.8 \\
1\end{array}$ & 0.12335 & $\begin{array}{c}0.002 \\
12\end{array}$ & $\begin{array}{c}6.1992 \\
4\end{array}$ & $\begin{array}{c}0.101 \\
91\end{array}$ & $\begin{array}{c}0.3645 \\
7\end{array}$ & $\begin{array}{c}0.005 \\
04\end{array}$ & 2005 & 13 & 2004 & 14 & 2004 & $\begin{array}{l}2 \\
4\end{array}$ & $0.05 \%$ \\
\hline $13 F R 12 \# 43$ & 37 & 36 & $\begin{array}{c}1.0 \\
3\end{array}$ & 0.13250 & $\begin{array}{c}0.009 \\
58\end{array}$ & $\begin{array}{c}5.9789 \\
8\end{array}$ & $\begin{array}{c}0.407 \\
55\end{array}$ & $\begin{array}{c}0.3273 \\
2\end{array}$ & $\begin{array}{c}0.013 \\
62\end{array}$ & 2131 & 64 & 1973 & 59 & 1825 & $\begin{array}{l}6 \\
6\end{array}$ & $16.77 \%$ \\
\hline 13FR12\#44 & 28 & 51 & $\begin{array}{c}0.5 \\
6\end{array}$ & 0.11606 & $\begin{array}{c}0.004 \\
29\end{array}$ & $\begin{array}{c}5.3030 \\
9\end{array}$ & $\begin{array}{c}0.186 \\
37\end{array}$ & $\begin{array}{c}0.3314 \\
4\end{array}$ & $\begin{array}{c}0.007 \\
34\end{array}$ & 1896 & 33 & 1869 & 30 & 1845 & $\begin{array}{l}3 \\
6\end{array}$ & $2.76 \%$ \\
\hline 13FR12\#45 & 29 & 49 & $\begin{array}{c}0.6 \\
0\end{array}$ & 0.11635 & $\begin{array}{c}0.004 \\
24\end{array}$ & $\begin{array}{c}5.1131 \\
3\end{array}$ & $\begin{array}{c}0.176 \\
46\end{array}$ & $\begin{array}{c}0.3187 \\
6\end{array}$ & $\begin{array}{c}0.006 \\
93\end{array}$ & 1901 & 33 & 1838 & 29 & 1784 & $\begin{array}{l}3 \\
4\end{array}$ & $6.56 \%$ \\
\hline 13FR12\#46 & 140 & 154 & $\begin{array}{c}0.9 \\
1\end{array}$ & 0.11923 & $\begin{array}{c}0.002 \\
51\end{array}$ & $\begin{array}{c}6.1709 \\
3\end{array}$ & $\begin{array}{c}0.124 \\
21\end{array}$ & $\begin{array}{c}0.3754 \\
0\end{array}$ & $\begin{array}{c}0.005 \\
74\end{array}$ & 1945 & 17 & 2000 & 18 & 2055 & $\begin{array}{l}2 \\
7\end{array}$ & $-5.35 \%$ \\
\hline 13FR12\#47 & 97 & 103 & $\begin{array}{c}0.9 \\
4\end{array}$ & 0.13789 & $\begin{array}{c}0.003 \\
28\end{array}$ & $\begin{array}{c}7.4513 \\
6\end{array}$ & $\begin{array}{c}0.169 \\
26\end{array}$ & $\begin{array}{c}0.3919 \\
8\end{array}$ & $\begin{array}{c}0.006 \\
73\end{array}$ & 2201 & 19 & 2167 & 20 & 2132 & $\begin{array}{l}3 \\
1\end{array}$ & $3.24 \%$ \\
\hline 13FR12\#48 & 217 & 139 & $\begin{array}{c}1.5 \\
7\end{array}$ & 0.06223 & $\begin{array}{c}0.003 \\
26\end{array}$ & $\begin{array}{c}1.0934 \\
7\end{array}$ & $\begin{array}{c}0.054 \\
70\end{array}$ & $\begin{array}{c}0.1274 \\
6\end{array}$ & $\begin{array}{c}0.002 \\
74\end{array}$ & 682 & 70 & 750 & 27 & 773 & $\begin{array}{l}1 \\
6\end{array}$ & $-2.98 \%$ \\
\hline 13FR12\#49 & 88 & 95 & $\begin{array}{c}0.9 \\
3\end{array}$ & 0.06088 & $\begin{array}{c}0.003 \\
49\end{array}$ & $\begin{array}{c}1.0482 \\
6\end{array}$ & $\begin{array}{c}0.057 \\
46\end{array}$ & $\begin{array}{c}0.1248 \\
9\end{array}$ & $\begin{array}{c}0.002 \\
86\end{array}$ & 635 & 79 & 728 & 28 & 759 & $\begin{array}{l}1 \\
6\end{array}$ & $-4.08 \%$ \\
\hline
\end{tabular}




\begin{tabular}{|c|c|c|c|c|c|c|c|c|c|c|c|c|c|c|c|c|}
\hline 13FR12\#50 & 38 & 110 & $\begin{array}{c}0.3 \\
5\end{array}$ & 0.19224 & $\begin{array}{c}0.003 \\
07\end{array}$ & $\begin{array}{c}14.350 \\
59\end{array}$ & $\begin{array}{c}0.222 \\
86\end{array}$ & $\begin{array}{c}0.5414 \\
5\end{array}$ & $\begin{array}{c}0.008 \\
07\end{array}$ & 2761 & 11 & 2773 & 15 & 2790 & $\begin{array}{l}3 \\
4\end{array}$ & $-1.04 \%$ \\
\hline 13FR12\#51 & 138 & 150 & $\begin{array}{c}0.9 \\
2\end{array}$ & 0.12638 & $\begin{array}{c}0.002 \\
61\end{array}$ & $\begin{array}{c}6.6697 \\
6\end{array}$ & $\begin{array}{c}0.132 \\
02\end{array}$ & $\begin{array}{c}0.3827 \\
8\end{array}$ & $\begin{array}{c}0.005 \\
88\end{array}$ & 2048 & 16 & 2069 & 17 & 2089 & $\begin{array}{l}2 \\
7\end{array}$ & $-1.96 \%$ \\
\hline 13FR12\#52 & 36 & 92 & $\begin{array}{c}0.3 \\
9\end{array}$ & 0.05532 & $\begin{array}{c}0.003 \\
49\end{array}$ & $\begin{array}{c}0.8428 \\
3\end{array}$ & $\begin{array}{c}0.050 \\
87\end{array}$ & $\begin{array}{c}0.1105 \\
0\end{array}$ & $\begin{array}{c}0.002 \\
62\end{array}$ & 425 & 92 & 621 & 28 & 676 & 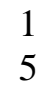 & $-8.14 \%$ \\
\hline 13FR12\#53 & 142 & 498 & $\begin{array}{c}0.2 \\
9\end{array}$ & 0.18163 & $\begin{array}{c}0.001 \\
60\end{array}$ & $\begin{array}{c}12.994 \\
11\end{array}$ & $\begin{array}{c}0.112 \\
18\end{array}$ & $\begin{array}{c}0.5188 \\
9\end{array}$ & $\begin{array}{c}0.005 \\
95\end{array}$ & 2668 & & & 8 & 2694 & $\begin{array}{l}2 \\
5\end{array}$ & $-0.97 \%$ \\
\hline 13FR12\#54 & 174 & 231 & $\begin{array}{c}0.7 \\
6\end{array}$ & 0.10147 & $\begin{array}{c}0.010 \\
28\end{array}$ & $\begin{array}{c}4.1532 \\
5\end{array}$ & $\begin{array}{c}0.410 \\
35\end{array}$ & $\begin{array}{c}0.2968 \\
5\end{array}$ & $\begin{array}{c}0.006 \\
59\end{array}$ & & & & 81 & 1676 & 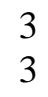 & $-1.49 \%$ \\
\hline 13FR12\#55 & 96 & 125 & $\begin{array}{c}0.7 \\
6\end{array}$ & 0.05769 & $\begin{array}{c}0.003 \\
39\end{array}$ & $\begin{array}{c}0.7147 \\
3\end{array}$ & $\begin{array}{c}0.040 \\
07\end{array}$ & $\begin{array}{c}0.0898 \\
6\end{array}$ & $\begin{array}{c}0.002 \\
02\end{array}$ & 518 & 84 & 548 & 24 & 555 & $\begin{array}{l}1 \\
2\end{array}$ & $-1.26 \%$ \\
\hline 13FR12\#56 & 110 & 51 & $\begin{array}{c}2.1 \\
5\end{array}$ & 0.06573 & $\begin{array}{c}0.004 \\
64\end{array}$ & $\begin{array}{c}0.9849 \\
4\end{array}$ & $\begin{array}{c}0.066 \\
10\end{array}$ & $\begin{array}{c}0.1086 \\
9\end{array}$ & $\begin{array}{c}0.002 \\
99\end{array}$ & 798 & 95 & 696 & 34 & 665 & $\begin{array}{l}1 \\
7\end{array}$ & $4.66 \%$ \\
\hline 13FR12\#57 & 62 & 197 & $\begin{array}{c}0.3 \\
1\end{array}$ & 0.11294 & $\begin{array}{c}0.002 \\
99\end{array}$ & $\begin{array}{l}5.0567 \\
3\end{array}$ & $\begin{array}{c}0.113 \\
22\end{array}$ & $\begin{array}{c}0.3247 \\
3\end{array}$ & $\begin{array}{c}0.004 \\
61\end{array}$ & 1847 & 49 & 1829 & 19 & 1813 & $\begin{array}{l}2 \\
2\end{array}$ & $1.88 \%$ \\
\hline 13FR12\#58 & 96 & 130 & $\begin{array}{c}0.7 \\
4\end{array}$ & 0.11460 & $\begin{array}{c}0.002 \\
47\end{array}$ & $\begin{array}{l}5.2066 \\
3\end{array}$ & $\begin{array}{c}0.106 \\
85\end{array}$ & $\begin{array}{c}0.3295 \\
1\end{array}$ & $\begin{array}{c}0.005 \\
01\end{array}$ & 1874 & 18 & 1854 & 17 & 1836 & 4 & $2.07 \%$ \\
\hline $13 F R 12 \# 59$ & 205 & 449 & $\begin{array}{c}0.4 \\
6\end{array}$ & 0.15162 & $\begin{array}{c}0.002 \\
23\end{array}$ & $\begin{array}{c}6.9470 \\
4\end{array}$ & $\begin{array}{c}0.096 \\
65\end{array}$ & $\begin{array}{c}0.3323 \\
0\end{array}$ & $\begin{array}{c}0.004 \\
39\end{array}$ & 2364 & 11 & 2105 & 12 & 1850 & $\begin{array}{l}2 \\
1\end{array}$ & $27.78 \%$ \\
\hline 13FR12\#60 & 276 & 389 & $\begin{array}{c}0.7 \\
1\end{array}$ & 0.06022 & $\begin{array}{c}0.001 \\
81\end{array}$ & $\begin{array}{c}0.8005 \\
0\end{array}$ & $\begin{array}{c}0.022 \\
89\end{array}$ & $\begin{array}{c}0.0964 \\
1\end{array}$ & $\begin{array}{c}0.001 \\
42\end{array}$ & 611 & 37 & 597 & 13 & 593 & 8 & $0.67 \%$ \\
\hline 13FR12\#61 & 69 & 83 & $\begin{array}{c}0.8 \\
4\end{array}$ & 0.06099 & $\begin{array}{c}0.004 \\
23\end{array}$ & $\begin{array}{c}0.8180 \\
7\end{array}$ & $\begin{array}{c}0.054 \\
23\end{array}$ & $\begin{array}{c}0.0972 \\
8\end{array}$ & $\begin{array}{c}0.002 \\
53\end{array}$ & 639 & 98 & 607 & 30 & 598 & 5 & $1.51 \%$ \\
\hline 13FR12\#62 & 71 & 458 & $\begin{array}{c}0.1 \\
5\end{array}$ & 0.05673 & $\begin{array}{c}0.001 \\
94\end{array}$ & $\begin{array}{c}0.5755 \\
9\end{array}$ & $\begin{array}{c}0.018 \\
82\end{array}$ & $\begin{array}{c}0.0735 \\
8\end{array}$ & $\begin{array}{c}0.001 \\
14\end{array}$ & 481 & 45 & 462 & 12 & 458 & 7 & $0.87 \%$ \\
\hline 13FR12\#63 & 277 & 306 & $\begin{array}{c}0.9 \\
0\end{array}$ & 0.11409 & $\begin{array}{c}0.001 \\
84\end{array}$ & $\begin{array}{c}5.6505 \\
2\end{array}$ & $\begin{array}{c}0.087 \\
26\end{array}$ & $\begin{array}{c}0.3591 \\
9\end{array}$ & $\begin{array}{c}0.004 \\
75\end{array}$ & 1866 & 12 & 1924 & 13 & 1978 & 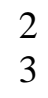 & $-5.66 \%$ \\
\hline 13FR12\#64 & 64 & 90 & $\begin{array}{c}0.7 \\
1\end{array}$ & 0.10059 & $\begin{array}{c}0.004 \\
66\end{array}$ & $\begin{array}{c}3.9629 \\
3\end{array}$ & $\begin{array}{c}0.169 \\
47\end{array}$ & $\begin{array}{c}0.2857 \\
4\end{array}$ & $\begin{array}{c}0.005 \\
13\end{array}$ & 1635 & 88 & 1627 & 35 & 1620 & 2 & $0.93 \%$ \\
\hline 13FR12\#65 & 59 & 90 & $\begin{array}{c}0.6 \\
6\end{array}$ & 0.11555 & $\begin{array}{c}0.005 \\
24\end{array}$ & $\begin{array}{c}5.0507 \\
6\end{array}$ & $\begin{array}{c}0.207 \\
71\end{array}$ & $\begin{array}{c}0.3170 \\
2\end{array}$ & $\begin{array}{c}0.006 \\
06\end{array}$ & 1888 & 84 & 1828 & 35 & 1775 & $\begin{array}{l}3 \\
0\end{array}$ & $6.37 \%$ \\
\hline 13FR12\#66 & 48 & 62 & $\begin{array}{c}0.7 \\
7\end{array}$ & 0.12001 & $\begin{array}{c}0.003 \\
59\end{array}$ & $\begin{array}{c}5.5290 \\
2\end{array}$ & $\begin{array}{c}0.157 \\
08\end{array}$ & $\begin{array}{c}0.3341 \\
1\end{array}$ & $\begin{array}{c}0.006 \\
33\end{array}$ & 1956 & 26 & 1905 & 24 & 1858 & $\begin{array}{l}3 \\
1\end{array}$ & $5.27 \%$ \\
\hline 13FR12\#67 & 41 & 102 & 0.4 & 0.12781 & 0.002 & 6.6466 & 0.132 & 0.3771 & 0.005 & 2068 & 16 & 2066 & 18 & 2063 & 2 & $0.24 \%$ \\
\hline
\end{tabular}




\begin{tabular}{|c|c|c|c|c|c|c|c|c|c|c|c|c|c|c|c|c|}
\hline & & & 0 & & 66 & 6 & 02 & 5 & 81 & & & & & & 7 & \\
\hline 13FR12\#68 & 29 & 84 & $\begin{array}{c}0.3 \\
5\end{array}$ & 0.05874 & $\begin{array}{c}0.004 \\
25\end{array}$ & $\begin{array}{c}0.6899 \\
7\end{array}$ & $\begin{array}{c}0.047 \\
87\end{array}$ & $\begin{array}{c}0.0851 \\
8\end{array}$ & $\begin{array}{c}0.002 \\
18\end{array}$ & 557 & $\begin{array}{c}10 \\
7\end{array}$ & 533 & 29 & 527 & $\begin{array}{l}1 \\
3\end{array}$ & $1.14 \%$ \\
\hline 13FR12\#69 & 91 & 192 & $\begin{array}{c}0.4 \\
7\end{array}$ & 0.20341 & $\begin{array}{c}0.002 \\
81\end{array}$ & $\begin{array}{c}15.193 \\
63\end{array}$ & $\begin{array}{c}0.204 \\
46\end{array}$ & $\begin{array}{c}0.5416 \\
8\end{array}$ & $\begin{array}{c}0.007 \\
53\end{array}$ & 2854 & 10 & 2827 & 13 & 2790 & $\begin{array}{l}3 \\
1\end{array}$ & $2.29 \%$ \\
\hline 13FR12\#70 & 101 & 236 & $\begin{array}{c}0.4 \\
3\end{array}$ & 0.05987 & $\begin{array}{c}0.002 \\
30\end{array}$ & $\begin{array}{c}0.7312 \\
9\end{array}$ & $\begin{array}{c}0.026 \\
81\end{array}$ & $\begin{array}{c}0.0885 \\
7\end{array}$ & $\begin{array}{c}0.001 \\
50\end{array}$ & 599 & 50 & & 16 & 547 & 9 & $1.83 \%$ \\
\hline 13FR12\#71 & 66 & 355 & $\begin{array}{c}0.1 \\
9\end{array}$ & 0.12583 & $\begin{array}{c}0.001 \\
77\end{array}$ & $\begin{array}{c}6.1763 \\
9\end{array}$ & $\begin{array}{c}0.083 \\
31\end{array}$ & $\begin{array}{c}0.3559 \\
4\end{array}$ & $\begin{array}{c}0.004 \\
52\end{array}$ & 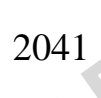 & & & 12 & 1963 & $\begin{array}{l}2 \\
1\end{array}$ & $3.97 \%$ \\
\hline 13FR12\#72 & 89 & 80 & $\begin{array}{c}1.1 \\
1\end{array}$ & 0.12217 & $\begin{array}{c}0.003 \\
07\end{array}$ & $\begin{array}{c}6.0527 \\
4\end{array}$ & $\begin{array}{c}0.145 \\
22\end{array}$ & $\begin{array}{c}0.3592 \\
7\end{array}$ & $\begin{array}{c}0.006 \\
12\end{array}$ & & 21 & 1983 & 21 & 1979 & $\begin{array}{l}2 \\
9\end{array}$ & $0.45 \%$ \\
\hline 13FR12\#73 & 55 & 62 & $\begin{array}{c}0.8 \\
9\end{array}$ & 0.11761 & $\begin{array}{c}0.003 \\
60\end{array}$ & $\begin{array}{c}5.7147 \\
1\end{array}$ & $\begin{array}{c}0.166 \\
51\end{array}$ & $\begin{array}{c}0.3523 \\
7\end{array}$ & 0.006 & 1920 & 27 & 1934 & 25 & 1946 & $\begin{array}{l}3 \\
2\end{array}$ & $-1.34 \%$ \\
\hline 13FR12\#74 & 119 & 181 & $\begin{array}{c}0.6 \\
6\end{array}$ & 0.05479 & $\begin{array}{c}0.002 \\
25\end{array}$ & $\begin{array}{c}0.7185 \\
9\end{array}$ & $\begin{array}{c}0.028 \\
22\end{array}$ & $\begin{array}{c}0.0951 \\
0\end{array}$ & $\begin{array}{c}0.001 \\
64\end{array}$ & 404 & 57 & 550 & 17 & 586 & $\begin{array}{l}1 \\
0\end{array}$ & $-6.14 \%$ \\
\hline 13FR12\#75 & 63 & 106 & $\begin{array}{c}0.6 \\
0\end{array}$ & 0.19552 & $\begin{array}{c}0.003 \\
57\end{array}$ & $\begin{array}{c}13.417 \\
26\end{array}$ & $\begin{array}{c}0.235 \\
65\end{array}$ & $\begin{array}{c}0.4976 \\
2\end{array}$ & $\begin{array}{c}0.007 \\
98\end{array}$ & 2789 & 13 & 2709 & 17 & 2604 & $\begin{array}{l}3 \\
4\end{array}$ & $7.10 \%$ \\
\hline 13FR12\#76 & 83 & 155 & $\begin{array}{c}0.5 \\
4\end{array}$ & 0.06214 & $\begin{array}{c}0.002 \\
84\end{array}$ & $\begin{array}{c}0.8235 \\
4\end{array}$ & $\begin{array}{c}0.035 \\
82\end{array}$ & $\begin{array}{c}0.0961 \\
0\end{array}$ & $\begin{array}{c}0.001 \\
84\end{array}$ & 679 & 60 & 610 & 20 & 592 & $\begin{array}{l}1 \\
1\end{array}$ & $3.04 \%$ \\
\hline 13FR12\#77 & 62 & 78 & $\begin{array}{c}0.8 \\
0\end{array}$ & 0.07045 & $\begin{array}{c}0.005 \\
39\end{array}$ & $\begin{array}{c}1.0146 \\
4\end{array}$ & $\begin{array}{c}0.073 \\
43\end{array}$ & $\begin{array}{c}0.1044 \\
3\end{array}$ & $\begin{array}{c}0.003 \\
21\end{array}$ & 941 & 98 & 711 & 37 & 640 & $\begin{array}{l}1 \\
9\end{array}$ & $11.09 \%$ \\
\hline 13FR12\#78 & 118 & 171 & $\begin{array}{c}0.6 \\
9\end{array}$ & 0.06215 & $\begin{array}{c}0.003 \\
15\end{array}$ & $\begin{array}{c}0.8123 \\
3\end{array}$ & $\begin{array}{c}0.039 \\
21\end{array}$ & $\begin{array}{c}0.0947 \\
8\end{array}$ & $\begin{array}{c}0.001 \\
96\end{array}$ & 679 & 68 & 604 & 22 & 584 & $\begin{array}{l}1 \\
2\end{array}$ & $3.42 \%$ \\
\hline 13FR12\#79 & 59 & 96 & $\begin{array}{c}0.6 \\
2\end{array}$ & 0.18180 & $\begin{array}{c}0.003 \\
13\end{array}$ & $\begin{array}{c}12.556 \\
04\end{array}$ & $\begin{array}{c}0.209 \\
10\end{array}$ & $\begin{array}{c}0.5008 \\
0\end{array}$ & $\begin{array}{c}0.007 \\
64\end{array}$ & 2669 & 12 & 2647 & 16 & 2617 & $\begin{array}{l}3 \\
3\end{array}$ & $1.99 \%$ \\
\hline 13FR12\#80 & 85 & 143 & $\begin{array}{c}0.6 \\
0\end{array}$ & 0.13351 & $\begin{array}{c}0.002 \\
22\end{array}$ & $\begin{array}{c}7.3870 \\
5\end{array}$ & $\begin{array}{c}0.117 \\
77\end{array}$ & $\begin{array}{c}0.4012 \\
1\end{array}$ & $\begin{array}{c}0.005 \\
56\end{array}$ & 2145 & 12 & 2159 & 14 & 2175 & $\begin{array}{l}2 \\
6\end{array}$ & $-1.38 \%$ \\
\hline 13FR12\#81 & 37 & 31 & $\begin{array}{c}1.2 \\
1\end{array}$ & 0.11283 & $\begin{array}{c}0.004 \\
51\end{array}$ & $\begin{array}{c}5.2872 \\
9\end{array}$ & $\begin{array}{c}0.201 \\
28\end{array}$ & $\begin{array}{c}0.3397 \\
9\end{array}$ & $\begin{array}{c}0.007 \\
88\end{array}$ & 1845 & 37 & 1867 & 33 & 1886 & $\begin{array}{l}3 \\
8\end{array}$ & $-2.17 \%$ \\
\hline 13FR12\#82 & 107 & 338 & $\begin{array}{c}0.3 \\
2\end{array}$ & 0.06048 & $\begin{array}{c}0.001 \\
88\end{array}$ & $\begin{array}{c}0.8445 \\
1\end{array}$ & $\begin{array}{c}0.025 \\
04\end{array}$ & $\begin{array}{c}0.1012 \\
4\end{array}$ & $\begin{array}{c}0.001 \\
52\end{array}$ & 621 & 38 & 622 & 14 & 622 & 9 & $0.00 \%$ \\
\hline 13FR12\#83 & 63 & 72 & $\begin{array}{c}0.8 \\
8\end{array}$ & 0.06557 & $\begin{array}{c}0.003 \\
67\end{array}$ & $\begin{array}{c}1.0331 \\
5\end{array}$ & $\begin{array}{c}0.055 \\
03\end{array}$ & $\begin{array}{c}0.1142 \\
5\end{array}$ & $\begin{array}{c}0.002 \\
61\end{array}$ & 793 & 73 & 721 & 27 & 697 & $\begin{array}{l}1 \\
5\end{array}$ & $3.44 \%$ \\
\hline 13FR12\#84 & 22 & 50 & $\begin{array}{c}0.4 \\
5\end{array}$ & 0.06515 & $\begin{array}{c}0.004 \\
80\end{array}$ & $\begin{array}{c}0.9352 \\
4\end{array}$ & $\begin{array}{c}0.065 \\
56\end{array}$ & $\begin{array}{c}0.1040 \\
8\end{array}$ & $\begin{array}{c}0.002 \\
95\end{array}$ & 779 & $\begin{array}{c}10 \\
0\end{array}$ & 670 & 34 & 638 & $\begin{array}{l}1 \\
7\end{array}$ & $5.02 \%$ \\
\hline
\end{tabular}




\begin{tabular}{|c|c|c|c|c|c|c|c|c|c|c|c|c|c|c|c|c|}
\hline 13FR12\#85 & 22 & 28 & $\begin{array}{c}0.7 \\
8\end{array}$ & 0.11674 & $\begin{array}{c}0.005 \\
31\end{array}$ & $\begin{array}{c}5.2398 \\
4\end{array}$ & $\begin{array}{c}0.226 \\
06\end{array}$ & $\begin{array}{c}0.3254 \\
3\end{array}$ & $\begin{array}{c}0.008 \\
47\end{array}$ & 1907 & 42 & 1859 & 37 & 1816 & $\begin{array}{l}4 \\
1\end{array}$ & $5.01 \%$ \\
\hline 13FR12\#86 & 18 & 18 & $\begin{array}{c}0.9 \\
8\end{array}$ & 0.05573 & $\begin{array}{c}0.008 \\
66\end{array}$ & $\begin{array}{c}0.7063 \\
5\end{array}$ & $\begin{array}{c}0.106 \\
28\end{array}$ & $\begin{array}{c}0.0919 \\
0\end{array}$ & $\begin{array}{c}0.004 \\
19\end{array}$ & 442 & $\begin{array}{c}25 \\
4\end{array}$ & 543 & 63 & 567 & $\begin{array}{l}2 \\
5\end{array}$ & $-4.23 \%$ \\
\hline 13FR12\#87 & 216 & 250 & $\begin{array}{c}0.8 \\
6\end{array}$ & 0.06134 & $\begin{array}{c}0.002 \\
35\end{array}$ & $\begin{array}{c}0.8037 \\
1\end{array}$ & $\begin{array}{c}0.029 \\
37\end{array}$ & $\begin{array}{c}0.0950 \\
0\end{array}$ & $\begin{array}{c}0.001 \\
61\end{array}$ & 651 & 50 & 599 & 17 & 585 & 9 & $2.39 \%$ \\
\hline 13FR12\#88 & 19 & 23 & $\begin{array}{c}0.8 \\
0\end{array}$ & 0.11739 & $\begin{array}{c}0.005 \\
22\end{array}$ & $\begin{array}{c}5.5060 \\
5\end{array}$ & $\begin{array}{c}0.232 \\
77\end{array}$ & $\begin{array}{c}0.3400 \\
6\end{array}$ & $\begin{array}{c}0.008 \\
79\end{array}$ & 1917 & 4 & & 36 & 1887 & $\begin{array}{l}4 \\
2\end{array}$ & $1.59 \%$ \\
\hline 13FR12\#89 & 83 & 156 & $\begin{array}{c}0.5 \\
3\end{array}$ & 0.10149 & $\begin{array}{c}0.003 \\
59\end{array}$ & $\begin{array}{c}3.7147 \\
6\end{array}$ & $\begin{array}{c}0.118 \\
49\end{array}$ & $\begin{array}{c}0.2654 \\
6\end{array}$ & $\begin{array}{c}0.004 \\
06\end{array}$ & 1652 & & 侸 & 26 & 1518 & $\begin{array}{l}2 \\
1\end{array}$ & $8.83 \%$ \\
\hline 13FR12\#90 & 66 & 108 & $\begin{array}{c}0.6 \\
1\end{array}$ & 0.23108 & $\begin{array}{c}0.003 \\
06\end{array}$ & $\begin{array}{c}18.819 \\
25\end{array}$ & $\begin{array}{c}0.244 \\
12\end{array}$ & $\begin{array}{c}0.5904 \\
6\end{array}$ & $\begin{array}{c}0.008 \\
22\end{array}$ & 3060 & 10 & 3033 & 13 & 2991 & $\begin{array}{l}3 \\
3\end{array}$ & $2.31 \%$ \\
\hline 13FR12\#91 & 4 & 558 & $\begin{array}{c}0.0 \\
1\end{array}$ & 0.12383 & $\begin{array}{c}0.001 \\
24\end{array}$ & $\begin{array}{c}6.4366 \\
9\end{array}$ & $\begin{array}{c}0.062 \\
60\end{array}$ & $\begin{array}{c}0.3768 \\
6\end{array}$ & $\begin{array}{c}0.004 \\
31\end{array}$ & 2012 & 9 & 2037 & 9 & 2062 & $\begin{array}{l}2 \\
0\end{array}$ & $-2.42 \%$ \\
\hline 13FR12\#92 & 122 & 95 & $\begin{array}{c}1.2 \\
9\end{array}$ & 0.11288 & $\begin{array}{c}0.004 \\
09\end{array}$ & $\begin{array}{c}4.9835 \\
8\end{array}$ & $\begin{array}{c}0.171 \\
46\end{array}$ & $\begin{array}{c}0.3200 \\
9\end{array}$ & $\begin{array}{c}0.006 \\
85\end{array}$ & 1846 & 33 & 1817 & 29 & 1790 & $\begin{array}{l}3 \\
3\end{array}$ & $3.13 \%$ \\
\hline 13FR12\#93 & 798 & 458 & $\begin{array}{c}1.7 \\
4\end{array}$ & 0.06272 & $\begin{array}{c}0.004 \\
89\end{array}$ & $\begin{array}{c}0.7120 \\
1\end{array}$ & $\begin{array}{c}0.054 \\
18\end{array}$ & $\begin{array}{c}0.0823 \\
3\end{array}$ & $\begin{array}{c}0.001 \\
38\end{array}$ & 699 & $\begin{array}{c}17 \\
1\end{array}$ & 546 & 32 & 510 & 8 & $7.06 \%$ \\
\hline 13FR12\#94 & 537 & 223 & $\begin{array}{c}2.4 \\
0\end{array}$ & 0.06250 & $\begin{array}{c}0.002 \\
24\end{array}$ & $\begin{array}{c}0.8302 \\
1\end{array}$ & $\begin{array}{l}0.028 \\
32\end{array}$ & $\begin{array}{c}0.0963 \\
0\end{array}$ & $\begin{array}{c}0.001 \\
58\end{array}$ & 691 & 45 & 614 & 16 & 593 & 9 & $3.54 \%$ \\
\hline 13FR12\#95 & 101 & 112 & $\begin{array}{c}0.9 \\
0\end{array}$ & 0.09064 & $\begin{array}{c}0.004 \\
41\end{array}$ & $\begin{array}{c}0.9322 \\
2\end{array}$ & $\begin{array}{c}0.042 \\
16\end{array}$ & $\begin{array}{c}0.0745 \\
6\end{array}$ & $\begin{array}{c}0.001 \\
68\end{array}$ & 1439 & 52 & 669 & 22 & 464 & $\begin{array}{l}1 \\
0\end{array}$ & $44.18 \%$ \\
\hline 13FR12\#96 & 181 & 238 & $\begin{array}{c}0.7 \\
6\end{array}$ & 0.05923 & $\begin{array}{c}0.001 \\
95\end{array}$ & $\begin{array}{c}0.8087 \\
0\end{array}$ & $\begin{array}{c}0.025 \\
41\end{array}$ & $\begin{array}{c}0.0989 \\
8\end{array}$ & $\begin{array}{c}0.001 \\
53\end{array}$ & 576 & 42 & 602 & 14 & 608 & 9 & $-0.99 \%$ \\
\hline 13FR12\#97 & 259 & 171 & $\begin{array}{c}1.5 \\
2\end{array}$ & 0.06082 & $\begin{array}{c}0.002 \\
94\end{array}$ & $\begin{array}{c}0.9369 \\
2\end{array}$ & $\begin{array}{c}0.043 \\
20\end{array}$ & $\begin{array}{c}0.1116 \\
7\end{array}$ & $\begin{array}{c}0.002 \\
24\end{array}$ & 633 & 65 & 671 & 23 & 682 & $\begin{array}{l}1 \\
3\end{array}$ & $-1.61 \%$ \\
\hline 13FR12\#98 & 61 & 69 & $\begin{array}{c}0.8 \\
9\end{array}$ & 0.11759 & $\begin{array}{c}0.003 \\
55\end{array}$ & $\begin{array}{c}5.3568 \\
7\end{array}$ & $\begin{array}{c}0.153 \\
46\end{array}$ & $\begin{array}{c}0.3302 \\
6\end{array}$ & $\begin{array}{c}0.006 \\
24\end{array}$ & 1920 & 26 & 1878 & 25 & 1840 & $\begin{array}{l}3 \\
0\end{array}$ & $4.35 \%$ \\
\hline 13FR12\#99 & 163 & 298 & $\begin{array}{c}0.5 \\
5\end{array}$ & 0.06246 & $\begin{array}{c}0.001 \\
75\end{array}$ & $\begin{array}{c}0.8233 \\
0\end{array}$ & $\begin{array}{c}0.021 \\
93\end{array}$ & $\begin{array}{c}0.0955 \\
6\end{array}$ & $\begin{array}{c}0.001 \\
37\end{array}$ & 690 & 33 & 610 & 12 & 588 & 8 & $3.74 \%$ \\
\hline $\begin{array}{l}\text { 13FR12\#10 } \\
0 \\
\text { 13FR13 }\end{array}$ & 25 & 67 & $\begin{array}{c}0.3 \\
7\end{array}$ & 0.10819 & $\begin{array}{c}0.002 \\
86\end{array}$ & $\begin{array}{c}4.9571 \\
2\end{array}$ & $\begin{array}{c}0.124 \\
76\end{array}$ & $\begin{array}{c}0.3321 \\
7\end{array}$ & $\begin{array}{c}0.005 \\
62\end{array}$ & 1769 & 23 & 1812 & 21 & 1849 & $\begin{array}{l}2 \\
7\end{array}$ & $-4.33 \%$ \\
\hline 13FR13\#01 & 161 & 371 & $\begin{array}{c}0.4 \\
3\end{array}$ & 0.05983 & $\begin{array}{c}0.001 \\
11\end{array}$ & $\begin{array}{c}0.6750 \\
1\end{array}$ & $\begin{array}{c}0.011 \\
47\end{array}$ & $\begin{array}{c}0.0818 \\
1\end{array}$ & $\begin{array}{c}0.001 \\
08\end{array}$ & 597 & 46 & 524 & 7 & 507 & 6 & $3.35 \%$ \\
\hline
\end{tabular}




\begin{tabular}{|c|c|c|c|c|c|c|c|c|c|c|c|c|c|c|c|c|}
\hline 13FR13\#02 & 105 & 154 & $\begin{array}{c}0.6 \\
9\end{array}$ & 0.06094 & $\begin{array}{c}0.001 \\
42\end{array}$ & $\begin{array}{c}0.8698 \\
2\end{array}$ & $\begin{array}{c}0.018 \\
61\end{array}$ & $\begin{array}{c}0.1034 \\
9\end{array}$ & $\begin{array}{c}0.001 \\
55\end{array}$ & 637 & 55 & 635 & 10 & 635 & 9 & $0.00 \%$ \\
\hline 13FR13\#03 & 89 & 923 & $\begin{array}{c}0.1 \\
0\end{array}$ & 0.05441 & $\begin{array}{c}0.000 \\
65\end{array}$ & $\begin{array}{c}0.5921 \\
4\end{array}$ & $\begin{array}{c}0.006 \\
63\end{array}$ & $\begin{array}{c}0.0789 \\
2\end{array}$ & $\begin{array}{c}0.000 \\
90\end{array}$ & 388 & 35 & 472 & 4 & 490 & 5 & $-3.67 \%$ \\
\hline 13FR13\#04 & 42 & 860 & $\begin{array}{c}0.0 \\
5\end{array}$ & 0.05926 & $\begin{array}{c}0.000 \\
91\end{array}$ & $\begin{array}{c}0.6856 \\
2\end{array}$ & $\begin{array}{c}0.009 \\
70\end{array}$ & $\begin{array}{c}0.0838 \\
9\end{array}$ & $\begin{array}{c}0.001 \\
03\end{array}$ & 577 & 40 & 530 & 6 & 519 & 6 & $2.12 \%$ \\
\hline 13FR13\#05 & 286 & 818 & $\begin{array}{c}0.3 \\
5\end{array}$ & 0.11466 & $\begin{array}{c}0.001 \\
63\end{array}$ & $\begin{array}{c}3.9176 \\
8\end{array}$ & $\begin{array}{c}0.035 \\
54\end{array}$ & $\begin{array}{c}0.2478 \\
2\end{array}$ & $\begin{array}{c}0.002 \\
72\end{array}$ & 1874 & & 517 & 7 & 1427 & $\begin{array}{l}1 \\
4\end{array}$ & $31.32 \%$ \\
\hline 13FR13\#06 & 122 & 196 & $\begin{array}{c}0.6 \\
2\end{array}$ & 0.05894 & $\begin{array}{c}0.002 \\
03\end{array}$ & $\begin{array}{c}0.6693 \\
4\end{array}$ & $\begin{array}{c}0.021 \\
14\end{array}$ & $\begin{array}{c}0.0823 \\
6\end{array}$ & $\begin{array}{c}0.001 \\
52\end{array}$ & & & 520 & 13 & 510 & 9 & $1.96 \%$ \\
\hline 13FR13\#07 & 125 & 343 & $\begin{array}{c}0.3 \\
6\end{array}$ & 0.13054 & $\begin{array}{c}0.001 \\
36\end{array}$ & $\begin{array}{c}6.3151 \\
3\end{array}$ & $\begin{array}{c}0.061 \\
42\end{array}$ & $\begin{array}{c}0.3508 \\
1\end{array}$ & $\begin{array}{c}0.004 \\
30\end{array}$ & 2105 & 23 & 2021 & 9 & 1938 & $\begin{array}{l}2 \\
1\end{array}$ & $8.62 \%$ \\
\hline 13FR13\#08 & 114 & 218 & $\begin{array}{c}0.5 \\
2\end{array}$ & 0.05474 & $\begin{array}{c}0.002 \\
46\end{array}$ & $\begin{array}{c}0.5647 \\
9\end{array}$ & $\begin{array}{c}0.023 \\
78\end{array}$ & $\begin{array}{c}0.0748 \\
3\end{array}$ & $\begin{array}{c}0.001 \\
19\end{array}$ & 402 & $\begin{array}{c}10 \\
3\end{array}$ & 455 & 15 & 465 & 7 & $-2.15 \%$ \\
\hline 13FR13\#09 & 143 & 705 & $\begin{array}{c}0.2 \\
0\end{array}$ & 0.05919 & $\begin{array}{c}0.000 \\
92\end{array}$ & $\begin{array}{c}0.6735 \\
7\end{array}$ & $\begin{array}{c}0.009 \\
65\end{array}$ & $\begin{array}{c}0.0825 \\
2\end{array}$ & $\begin{array}{c}0.001 \\
02\end{array}$ & 574 & 40 & 523 & 6 & 511 & 6 & $2.35 \%$ \\
\hline 13FR13\#10 & 247 & 1030 & $\begin{array}{c}0.2 \\
4\end{array}$ & 0.05393 & $\begin{array}{c}0.000 \\
75\end{array}$ & $\begin{array}{c}0.5851 \\
3\end{array}$ & $\begin{array}{c}0.007 \\
51\end{array}$ & $\begin{array}{c}0.0786 \\
9\end{array}$ & $\begin{array}{c}0.000 \\
93\end{array}$ & 368 & 39 & 468 & 5 & 488 & 6 & $-4.10 \%$ \\
\hline 13FR13\#11 & 255 & 584 & $\begin{array}{c}0.4 \\
4\end{array}$ & 0.05415 & $\begin{array}{c}0.000 \\
78\end{array}$ & 0.5857 & $\begin{array}{c}0.007 \\
85\end{array}$ & $\begin{array}{c}0.0784 \\
5\end{array}$ & $\begin{array}{c}0.000 \\
93\end{array}$ & 377 & 40 & 468 & 5 & 487 & 6 & $-3.90 \%$ \\
\hline 13FR13\#12 & 129 & 405 & $\begin{array}{c}0.3 \\
2\end{array}$ & 0.05960 & $\begin{array}{c}0.000 \\
87\end{array}$ & $\begin{array}{c}0.7878 \\
8\end{array}$ & $\begin{array}{c}0.010 \\
68\end{array}$ & $\begin{array}{c}0.0958 \\
8\end{array}$ & $\begin{array}{c}0.001 \\
16\end{array}$ & 589 & 38 & 590 & 6 & 590 & 7 & $0.00 \%$ \\
\hline 13FR13\#13 & 170 & 269 & $\begin{array}{c}0.6 \\
3\end{array}$ & 0.11683 & $\begin{array}{c}0.000 \\
98\end{array}$ & $\begin{array}{c}5.4368 \\
4\end{array}$ & $\begin{array}{c}0.043 \\
27\end{array}$ & $\begin{array}{c}0.3375 \\
0\end{array}$ & $\begin{array}{c}0.003 \\
82\end{array}$ & 1908 & 21 & 1891 & 7 & 1875 & $\begin{array}{l}1 \\
8\end{array}$ & $1.76 \%$ \\
\hline 13FR13\#14 & 110 & 759 & $\begin{array}{c}0.1 \\
5\end{array}$ & 005696 & $\begin{array}{c}0.000 \\
70\end{array}$ & $\begin{array}{c}0.5975 \\
9\end{array}$ & $\begin{array}{c}0.006 \\
82\end{array}$ & $\begin{array}{c}0.0760 \\
8\end{array}$ & $\begin{array}{c}0.000 \\
87\end{array}$ & 490 & 35 & 476 & 4 & 473 & 5 & $0.63 \%$ \\
\hline 13FR13\#15 & 125 & 492 & $\begin{array}{c}0.2 \\
5\end{array}$ & 0.05805 & $\begin{array}{c}0.000 \\
81\end{array}$ & $\begin{array}{c}0.6026 \\
9\end{array}$ & $\begin{array}{c}0.007 \\
77\end{array}$ & $\begin{array}{c}0.0753 \\
0\end{array}$ & $\begin{array}{c}0.000 \\
90\end{array}$ & 532 & 38 & 479 & 5 & 468 & 5 & $2.35 \%$ \\
\hline 13FR13\#16 & 471 & 1504 & $\begin{array}{c}0.3 \\
1\end{array}$ & 0.05784 & $\begin{array}{c}0.001 \\
04\end{array}$ & $\begin{array}{c}0.7287 \\
1\end{array}$ & $\begin{array}{c}0.010 \\
34\end{array}$ & $\begin{array}{c}0.0913 \\
7\end{array}$ & $\begin{array}{c}0.001 \\
01\end{array}$ & 524 & 40 & 556 & 6 & 564 & 6 & $-1.42 \%$ \\
\hline 13FR13\#17 & 64 & 245 & $\begin{array}{c}0.2 \\
6\end{array}$ & 0.05649 & $\begin{array}{c}0.004 \\
80\end{array}$ & $\begin{array}{c}0.5834 \\
9\end{array}$ & $\begin{array}{c}0.046 \\
00\end{array}$ & $\begin{array}{c}0.0749 \\
2\end{array}$ & $\begin{array}{c}0.002 \\
69\end{array}$ & 472 & $\begin{array}{c}19 \\
2\end{array}$ & 467 & 29 & 466 & $\begin{array}{l}1 \\
6\end{array}$ & $0.21 \%$ \\
\hline 13FR13\#18 & 197 & 702 & $\begin{array}{c}0.2 \\
8\end{array}$ & 0.05779 & $\begin{array}{c}0.001 \\
37\end{array}$ & $\begin{array}{c}0.5692 \\
4\end{array}$ & $\begin{array}{c}0.011 \\
54\end{array}$ & $\begin{array}{c}0.0714 \\
4\end{array}$ & $\begin{array}{c}0.000 \\
88\end{array}$ & 522 & 53 & 458 & 7 & 445 & 5 & $2.92 \%$ \\
\hline 13FR13\#19 & 127 & 271 & 0.4 & 0.05969 & 0.001 & 0.6764 & 0.011 & 0.0821 & 0.001 & 592 & 46 & 525 & 7 & 509 & 7 & $3.14 \%$ \\
\hline
\end{tabular}




\begin{tabular}{|c|c|c|c|c|c|c|c|c|c|c|c|c|c|c|c|c|}
\hline & & & 7 & & 11 & 5 & 55 & 9 & 10 & & & & & & & \\
\hline 13FR13\#20 & 81 & 787 & $\begin{array}{c}0.1 \\
0\end{array}$ & 0.05452 & $\begin{array}{c}0.000 \\
65\end{array}$ & $\begin{array}{c}0.5740 \\
7\end{array}$ & $\begin{array}{c}0.006 \\
42\end{array}$ & $\begin{array}{c}0.0763 \\
7\end{array}$ & $\begin{array}{c}0.000 \\
87\end{array}$ & 393 & 35 & 461 & 4 & 474 & 5 & $-2.74 \%$ \\
\hline 13FR13\#21 & 85 & 161 & $\begin{array}{c}0.5 \\
3\end{array}$ & 0.06185 & $\begin{array}{c}0.001 \\
39\end{array}$ & $\begin{array}{c}0.7803 \\
3\end{array}$ & $\begin{array}{c}0.015 \\
93\end{array}$ & $\begin{array}{c}0.0915 \\
1\end{array}$ & $\begin{array}{c}0.001 \\
35\end{array}$ & 669 & 53 & 586 & 9 & 564 & 8 & $3.90 \%$ \\
\hline 13FR13\#22 & 380 & 907 & $\begin{array}{c}0.4 \\
2\end{array}$ & 0.05736 & $\begin{array}{c}0.000 \\
78\end{array}$ & $\begin{array}{c}0.5893 \\
1\end{array}$ & $\begin{array}{c}0.007 \\
40\end{array}$ & $\begin{array}{c}0.0745 \\
1\end{array}$ & $\begin{array}{c}0.000 \\
88\end{array}$ & 505 & 37 & & 5 & 463 & 5 & $1.51 \%$ \\
\hline 13FR13\#23 & 367 & 733 & $\begin{array}{c}0.5 \\
0\end{array}$ & 0.05678 & $\begin{array}{c}0.000 \\
65\end{array}$ & $\begin{array}{c}0.5853 \\
0\end{array}$ & $\begin{array}{c}0.006 \\
29\end{array}$ & $\begin{array}{c}0.0747 \\
6\end{array}$ & $\begin{array}{c}0.000 \\
85\end{array}$ & 483 & & & 4 & 465 & 5 & $0.65 \%$ \\
\hline 13FR13\#24 & 136 & 650 & $\begin{array}{c}0.2 \\
1\end{array}$ & 0.05671 & $\begin{array}{c}0.000 \\
68\end{array}$ & $\begin{array}{c}0.6149 \\
7\end{array}$ & $\begin{array}{c}0.006 \\
83\end{array}$ & $\begin{array}{c}0.0786 \\
6\end{array}$ & $\begin{array}{c}0.000 \\
90\end{array}$ & & 35 & 487 & 4 & 488 & 5 & $-0.20 \%$ \\
\hline 13FR13\#25 & 107 & 341 & $\begin{array}{c}0.3 \\
1\end{array}$ & 0.07758 & $\begin{array}{c}0.001 \\
71\end{array}$ & $\begin{array}{c}1.5315 \\
8\end{array}$ & $\begin{array}{c}0.028 \\
13\end{array}$ & $\begin{array}{c}0.1431 \\
8\end{array}$ & $\begin{array}{c}0.001 \\
75\end{array}$ & 1136 & 45 & 943 & 11 & 863 & $\begin{array}{l}1 \\
0\end{array}$ & $9.27 \%$ \\
\hline 13FR13\#26 & 142 & 764 & $\begin{array}{c}0.1 \\
9\end{array}$ & 0.09406 & $\begin{array}{c}0.000 \\
75\end{array}$ & $\begin{array}{c}1.9573 \\
4\end{array}$ & $\begin{array}{c}0.014 \\
60\end{array}$ & $\begin{array}{c}0.1509 \\
4\end{array}$ & $\begin{array}{c}0.001 \\
65\end{array}$ & 1509 & 24 & 1101 & 5 & 906 & 9 & $21.52 \%$ \\
\hline 13FR13\#27 & 80 & 597 & $\begin{array}{c}0.1 \\
3\end{array}$ & 0.05616 & $\begin{array}{c}0.000 \\
95\end{array}$ & $\begin{array}{c}0.6422 \\
6\end{array}$ & $\begin{array}{c}0.010 \\
05\end{array}$ & $\begin{array}{c}0.0829 \\
5\end{array}$ & $\begin{array}{c}0.001 \\
06\end{array}$ & 459 & 44 & 504 & 6 & 514 & 6 & $-1.95 \%$ \\
\hline 13FR13\#28 & 147 & 462 & $\begin{array}{c}0.3 \\
2\end{array}$ & 0.05665 & $\begin{array}{c}0.000 \\
77\end{array}$ & $\begin{array}{c}0.5953 \\
9\end{array}$ & $\begin{array}{c}0.007 \\
47\end{array}$ & $\begin{array}{c}0.0762 \\
4\end{array}$ & $\begin{array}{c}0.000 \\
90\end{array}$ & 478 & 37 & 474 & 5 & 474 & 5 & $0.00 \%$ \\
\hline 13FR13\#29 & 48 & 419 & $\begin{array}{c}0.1 \\
1\end{array}$ & 0.14037 & $\begin{array}{c}0.002 \\
34\end{array}$ & $\begin{array}{c}2.9261 \\
8\end{array}$ & $\begin{array}{c}0.032 \\
80\end{array}$ & $\begin{array}{c}0.1511 \\
9\end{array}$ & $\begin{array}{c}0.001 \\
87\end{array}$ & 2232 & 30 & 1389 & 8 & 908 & $\begin{array}{l}1 \\
0\end{array}$ & $52.97 \%$ \\
\hline 13FR13\#30 & 78 & 545 & $\begin{array}{c}0.1 \\
4\end{array}$ & 0.05433 & $\begin{array}{c}0.000 \\
70\end{array}$ & $\begin{array}{c}0.5732 \\
7\end{array}$ & $\begin{array}{c}0.006 \\
87\end{array}$ & $\begin{array}{c}0.0765 \\
4\end{array}$ & $\begin{array}{c}0.000 \\
89\end{array}$ & 385 & 37 & 460 & 4 & 475 & 5 & $-3.16 \%$ \\
\hline 13FR13\#31 & 227 & 360 & $\begin{array}{c}0.6 \\
3\end{array}$ & 0.05645 & $\begin{array}{c}0.000 \\
81\end{array}$ & $\begin{array}{c}0.6053 \\
8\end{array}$ & $\begin{array}{c}0.008 \\
02\end{array}$ & $\begin{array}{c}0.0777 \\
9\end{array}$ & $\begin{array}{c}0.000 \\
93\end{array}$ & 470 & 39 & 481 & 5 & 483 & 6 & $-0.41 \%$ \\
\hline 13FR13\#32 & 82 & 719 & $\begin{array}{c}0.1 \\
1\end{array}$ & 0.05835 & $\begin{array}{c}0.000 \\
63\end{array}$ & $\begin{array}{c}0.6122 \\
4\end{array}$ & $\begin{array}{c}0.006 \\
22\end{array}$ & $\begin{array}{c}0.0761 \\
1\end{array}$ & $\begin{array}{c}0.000 \\
86\end{array}$ & 543 & 33 & 485 & 4 & 473 & 5 & $2.54 \%$ \\
\hline 13FR13\#33 & 119 & 679 & $\begin{array}{c}0.1 \\
7\end{array}$ & 0.05695 & $\begin{array}{c}0.000 \\
79\end{array}$ & $\begin{array}{c}0.6365 \\
1\end{array}$ & $\begin{array}{c}0.008 \\
21\end{array}$ & $\begin{array}{c}0.0810 \\
7\end{array}$ & $\begin{array}{c}0.000 \\
97\end{array}$ & 490 & 38 & 500 & 5 & 503 & 6 & $-0.60 \%$ \\
\hline 13FR13\#34 & 325 & 301 & $\begin{array}{c}1.0 \\
8\end{array}$ & 0.11468 & $\begin{array}{c}0.000 \\
85\end{array}$ & $\begin{array}{c}5.1355 \\
5\end{array}$ & $\begin{array}{c}0.036 \\
86\end{array}$ & $\begin{array}{c}0.3248 \\
4\end{array}$ & $\begin{array}{c}0.003 \\
59\end{array}$ & 1875 & 20 & 1842 & 6 & 1813 & $\begin{array}{l}1 \\
7\end{array}$ & $3.42 \%$ \\
\hline 13FR13\#35 & 88 & 636 & $\begin{array}{c}0.1 \\
4\end{array}$ & 0.09032 & $\begin{array}{c}0.001 \\
25\end{array}$ & $\begin{array}{c}2.2081 \\
1\end{array}$ & $\begin{array}{c}0.019 \\
03\end{array}$ & $\begin{array}{c}0.1773 \\
2\end{array}$ & $\begin{array}{c}0.001 \\
93\end{array}$ & 1432 & 27 & 1184 & 6 & 1052 & $\begin{array}{l}1 \\
1\end{array}$ & $36.12 \%$ \\
\hline 13FR13\#36 & 46 & 414 & $\begin{array}{c}0.1 \\
1\end{array}$ & 0.10202 & $\begin{array}{c}0.001 \\
60\end{array}$ & $\begin{array}{c}1.7396 \\
6\end{array}$ & $\begin{array}{c}0.018 \\
61\end{array}$ & $\begin{array}{c}0.1236 \\
7\end{array}$ & $\begin{array}{c}0.001 \\
42\end{array}$ & 1661 & 30 & 1023 & 7 & 752 & 8 & $36.04 \%$ \\
\hline
\end{tabular}




\begin{tabular}{|c|c|c|c|c|c|c|c|c|c|c|c|c|c|c|c|c|}
\hline 13FR13\#37 & 174 & 801 & $\begin{array}{c}0.2 \\
2\end{array}$ & 0.05690 & $\begin{array}{c}0.000 \\
86\end{array}$ & $\begin{array}{c}0.5855 \\
7\end{array}$ & $\begin{array}{c}0.008 \\
15\end{array}$ & $\begin{array}{c}0.0746 \\
6\end{array}$ & $\begin{array}{c}0.000 \\
92\end{array}$ & 488 & 40 & 468 & 5 & 464 & 6 & $0.86 \%$ \\
\hline 13FR13\#38 & 91 & 402 & $\begin{array}{c}0.2 \\
3\end{array}$ & 0.05588 & $\begin{array}{c}0.000 \\
86\end{array}$ & $\begin{array}{c}0.6191 \\
6\end{array}$ & $\begin{array}{c}0.008 \\
83\end{array}$ & $\begin{array}{c}0.0803 \\
7\end{array}$ & $\begin{array}{c}0.000 \\
99\end{array}$ & 448 & 41 & 489 & 6 & 498 & 6 & $-1.81 \%$ \\
\hline 13FR13\#39 & 186 & 188 & $\begin{array}{c}0.9 \\
9\end{array}$ & 0.11695 & $\begin{array}{c}0.000 \\
99\end{array}$ & $\begin{array}{c}5.1375 \\
7\end{array}$ & $\begin{array}{c}0.041 \\
42\end{array}$ & $\begin{array}{c}0.3186 \\
5\end{array}$ & $\begin{array}{c}0.003 \\
64\end{array}$ & 1910 & 21 & 1842 & 7 & 1783 & $\begin{array}{l}1 \\
8\end{array}$ & $7.12 \%$ \\
\hline 13FR13\#40 & 31 & 257 & $\begin{array}{c}0.1 \\
2\end{array}$ & 0.06042 & $\begin{array}{c}0.001 \\
29\end{array}$ & $\begin{array}{c}0.6728 \\
1\end{array}$ & $\begin{array}{c}0.011 \\
72\end{array}$ & $\begin{array}{c}0.0807 \\
7\end{array}$ & $\begin{array}{c}0.001 \\
00\end{array}$ & 619 & & & 7 & 501 & 6 & $4.19 \%$ \\
\hline 13FR13\#41 & 52 & 411 & $\begin{array}{c}0.1 \\
3\end{array}$ & 0.05679 & $\begin{array}{c}0.000 \\
81\end{array}$ & $\begin{array}{c}0.6592 \\
6\end{array}$ & $\begin{array}{c}0.008 \\
75\end{array}$ & $\begin{array}{c}0.0842 \\
1\end{array}$ & $\begin{array}{c}0.001 \\
02\end{array}$ & & & 514 & 5 & 521 & 6 & $-1.34 \%$ \\
\hline $13 F R 13 \# 42$ & 440 & 1242 & $\begin{array}{c}0.3 \\
5\end{array}$ & 0.07698 & $\begin{array}{c}0.001 \\
62\end{array}$ & $\begin{array}{c}0.6340 \\
1\end{array}$ & $\begin{array}{c}0.011 \\
02\end{array}$ & $\begin{array}{c}0.0597 \\
4\end{array}$ & $\begin{array}{c}0.000 \\
70\end{array}$ & 1121 & 43 & 499 & 7 & 374 & 4 & $33.42 \%$ \\
\hline 13FR13\#43 & 256 & 291 & $\begin{array}{c}0.8 \\
8\end{array}$ & 0.05473 & $\begin{array}{c}0.001 \\
25\end{array}$ & $\begin{array}{c}0.6415 \\
6\end{array}$ & $\begin{array}{c}0.013 \\
49\end{array}$ & $\begin{array}{c}0.0850 \\
4\end{array}$ & $\begin{array}{c}0.001 \\
24\end{array}$ & 401 & 56 & 503 & 8 & 526 & 7 & $-4.37 \%$ \\
\hline 13FR13\#44 & 237 & 422 & $\begin{array}{c}0.5 \\
6\end{array}$ & 0.06329 & $\begin{array}{c}0.000 \\
74\end{array}$ & $\begin{array}{c}0.8365 \\
7\end{array}$ & $\begin{array}{c}0.009 \\
15\end{array}$ & $\begin{array}{c}0.0958 \\
9\end{array}$ & $\begin{array}{c}0.001 \\
12\end{array}$ & 718 & 33 & 617 & 5 & 590 & 7 & $4.58 \%$ \\
\hline 13FR13\#45 & 69 & 344 & $\begin{array}{c}0.2 \\
0\end{array}$ & 0.16455 & $\begin{array}{c}0.002 \\
20\end{array}$ & $\begin{array}{c}6.1105 \\
4\end{array}$ & $\begin{array}{c}0.046 \\
05\end{array}$ & $\begin{array}{c}0.2693 \\
3\end{array}$ & $\begin{array}{c}0.002 \\
98\end{array}$ & 2503 & 23 & 1992 & 7 & 1537 & $\begin{array}{l}1 \\
5\end{array}$ & $62.85 \%$ \\
\hline 13FR13\#46 & 101 & 755 & $\begin{array}{c}0.1 \\
3\end{array}$ & 0.05673 & $\begin{array}{c}0.000 \\
77\end{array}$ & 0.6348 & $\begin{array}{c}0.007 \\
99\end{array}$ & $\begin{array}{c}0.0811 \\
8\end{array}$ & $\begin{array}{c}0.000 \\
97\end{array}$ & 481 & 38 & 499 & 5 & 503 & 6 & $-0.80 \%$ \\
\hline 13FR13\#47 & 56 & 534 & $\begin{array}{c}0.1 \\
1\end{array}$ & 0.05633 & $\begin{array}{c}0.000 \\
71\end{array}$ & $\begin{array}{c}0.6032 \\
3\end{array}$ & $\begin{array}{c}0.007 \\
09\end{array}$ & $\begin{array}{c}0.0776 \\
9\end{array}$ & $\begin{array}{c}0.000 \\
91\end{array}$ & 465 & 36 & 479 & 4 & 482 & 5 & $-0.62 \%$ \\
\hline 13FR13\#48 & 108 & 287 & $\begin{array}{c}0.3 \\
8\end{array}$ & 0.05709 & $\begin{array}{c}0.001 \\
15\end{array}$ & $\begin{array}{c}0.6348 \\
9\end{array}$ & $\begin{array}{c}0.011 \\
75\end{array}$ & $\begin{array}{c}0.0806 \\
8\end{array}$ & $\begin{array}{c}0.001 \\
10\end{array}$ & 495 & 50 & 499 & 7 & 500 & 7 & $-0.20 \%$ \\
\hline 13FR13\#49 & 122 & 246 & $\begin{array}{c}0.5 \\
0\end{array}$ & 0.06024 & $\begin{array}{c}0.001 \\
24\end{array}$ & $\begin{array}{c}0.7803 \\
5\end{array}$ & $\begin{array}{c}0.014 \\
74\end{array}$ & $\begin{array}{c}0.0939 \\
8\end{array}$ & $\begin{array}{c}0.001 \\
33\end{array}$ & 612 & 50 & 586 & 8 & 579 & 8 & $1.21 \%$ \\
\hline 13FR13\#50 & 312 & 561 & $\begin{array}{c}0.5 \\
6\end{array}$ & 0.05867 & $\begin{array}{c}0.001 \\
46\end{array}$ & $\begin{array}{c}0.7385 \\
3\end{array}$ & $\begin{array}{c}0.016 \\
10\end{array}$ & $\begin{array}{c}0.0913 \\
0\end{array}$ & $\begin{array}{c}0.001 \\
10\end{array}$ & 555 & 56 & 562 & 9 & 563 & 7 & $-0.18 \%$ \\
\hline 13FR13\#51 & 168 & 291 & $\begin{array}{c}0.5 \\
8\end{array}$ & 0.05831 & $\begin{array}{c}0.000 \\
73\end{array}$ & $\begin{array}{c}0.8629 \\
1\end{array}$ & $\begin{array}{c}0.010 \\
10\end{array}$ & $\begin{array}{c}0.1073 \\
6\end{array}$ & $\begin{array}{c}0.001 \\
26\end{array}$ & 541 & 35 & 632 & 6 & 657 & 7 & $-3.81 \%$ \\
\hline 13FR13\#52 & 271 & 469 & $\begin{array}{c}0.5 \\
8\end{array}$ & 0.07070 & $\begin{array}{c}0.000 \\
75\end{array}$ & $\begin{array}{c}0.7569 \\
4\end{array}$ & $\begin{array}{c}0.007 \\
44\end{array}$ & $\begin{array}{c}0.0776 \\
7\end{array}$ & $\begin{array}{c}0.000 \\
89\end{array}$ & 949 & 30 & 572 & 4 & 482 & 5 & $18.67 \%$ \\
\hline 13FR13\#53 & 242 & 435 & $\begin{array}{c}0.5 \\
6\end{array}$ & 0.05441 & $\begin{array}{c}0.000 \\
65\end{array}$ & $\begin{array}{c}0.5856 \\
3\end{array}$ & $\begin{array}{c}0.006 \\
59\end{array}$ & $\begin{array}{c}0.0780 \\
9\end{array}$ & $\begin{array}{c}0.000 \\
90\end{array}$ & 388 & 36 & 468 & 4 & 485 & 5 & $-3.51 \%$ \\
\hline 13FR13\#54 & 207 & 400 & 0.5 & 0.05627 & 0.000 & 0.6093 & 0.007 & 0.0785 & 0.000 & 463 & 36 & 483 & 4 & 487 & 5 & $-0.82 \%$ \\
\hline
\end{tabular}




\begin{tabular}{|c|c|c|c|c|c|c|c|c|c|c|c|c|c|c|c|c|}
\hline & & & 2 & & 70 & 2 & 08 & 5 & 92 & & & & & & & \\
\hline 13FR13\#55 & 325 & 299 & $\begin{array}{c}1.0 \\
9\end{array}$ & 0.06249 & $\begin{array}{c}0.000 \\
95\end{array}$ & $\begin{array}{c}0.9694 \\
6\end{array}$ & $\begin{array}{c}0.013 \\
70\end{array}$ & $\begin{array}{c}0.1125 \\
5\end{array}$ & $\begin{array}{c}0.001 \\
42\end{array}$ & 691 & 39 & 688 & 7 & 688 & 8 & $0.00 \%$ \\
\hline 13FR13\#56 & 102 & 137 & $\begin{array}{c}0.7 \\
4\end{array}$ & 0.05660 & $\begin{array}{c}0.001 \\
02\end{array}$ & $\begin{array}{c}0.7906 \\
1\end{array}$ & $\begin{array}{c}0.013 \\
22\end{array}$ & $\begin{array}{c}0.1013 \\
4\end{array}$ & $\begin{array}{c}0.001 \\
33\end{array}$ & 476 & 46 & 592 & 7 & 622 & 8 & $-4.82 \%$ \\
\hline 13FR13\#57 & 105 & 665 & $\begin{array}{c}0.1 \\
6\end{array}$ & 0.05836 & $\begin{array}{c}0.000 \\
56\end{array}$ & $\begin{array}{c}0.6349 \\
4\end{array}$ & $\begin{array}{c}0.005 \\
75\end{array}$ & $\begin{array}{c}0.0789 \\
3\end{array}$ & $\begin{array}{c}0.000 \\
88\end{array}$ & 543 & 21 & & 4 & 490 & 5 & $1.84 \%$ \\
\hline 13FR13\#58 & 169 & 416 & $\begin{array}{c}0.4 \\
1\end{array}$ & 0.05739 & $\begin{array}{c}0.000 \\
66\end{array}$ & $\begin{array}{c}0.6093 \\
1\end{array}$ & $\begin{array}{c}0.006 \\
58\end{array}$ & $\begin{array}{c}0.0770 \\
3\end{array}$ & $\begin{array}{c}0.000 \\
89\end{array}$ & 507 & & & 4 & 478 & 5 & $1.05 \%$ \\
\hline 13FR13\#59 & 68 & 95 & $\begin{array}{c}0.7 \\
2\end{array}$ & 0.12465 & $\begin{array}{c}0.001 \\
24\end{array}$ & $\begin{array}{c}6.3396 \\
3\end{array}$ & $\begin{array}{c}0.059 \\
67\end{array}$ & $\begin{array}{c}0.3689 \\
6\end{array}$ & $\begin{array}{c}0.004 \\
50\end{array}$ & & 22 & 2024 & 8 & 2025 & $\begin{array}{l}2 \\
1\end{array}$ & $-0.05 \%$ \\
\hline 13FR13\#60 & 156 & 425 & $\begin{array}{c}0.3 \\
7\end{array}$ & 0.05651 & $\begin{array}{c}0.000 \\
90\end{array}$ & $\begin{array}{c}0.6575 \\
1\end{array}$ & $\begin{array}{c}0.009 \\
70\end{array}$ & $\begin{array}{c}0.0844 \\
0\end{array}$ & 0.001 & 472 & 42 & 513 & 6 & 522 & 6 & $-1.72 \%$ \\
\hline 13FR13\#61 & 156 & 311 & $\begin{array}{c}0.5 \\
0\end{array}$ & 0.05671 & $\begin{array}{c}0.000 \\
69\end{array}$ & $\begin{array}{c}0.6665 \\
3\end{array}$ & $\begin{array}{c}0.007 \\
55\end{array}$ & $\begin{array}{c}0.0852 \\
7\end{array}$ & $\begin{array}{c}0.000 \\
99\end{array}$ & 480 & 35 & 519 & 5 & 528 & 6 & $-1.70 \%$ \\
\hline 13FR13\#62 & 82 & 311 & $\begin{array}{c}0.2 \\
6\end{array}$ & 0.05774 & $\begin{array}{c}0.001 \\
02\end{array}$ & $\begin{array}{c}0.6486 \\
3\end{array}$ & $\begin{array}{c}0.010 \\
51\end{array}$ & $\begin{array}{c}0.0815 \\
0\end{array}$ & $\begin{array}{c}0.001 \\
07\end{array}$ & 520 & 45 & 508 & 6 & 505 & 6 & $0.59 \%$ \\
\hline 13FR13\#63 & 77 & 118 & $\begin{array}{c}0.6 \\
5\end{array}$ & 0.06186 & $\begin{array}{c}0.001 \\
35\end{array}$ & $\begin{array}{c}0.6931 \\
7\end{array}$ & $\begin{array}{c}0.013 \\
87\end{array}$ & $\begin{array}{c}0.0812 \\
9\end{array}$ & $\begin{array}{c}0.001 \\
18\end{array}$ & 669 & 52 & 535 & 8 & 504 & 7 & $6.15 \%$ \\
\hline 13FR13\#64 & 396 & 630 & $\begin{array}{c}0.6 \\
3\end{array}$ & 0.08626 & $\begin{array}{c}0.000 \\
72\end{array}$ & $\begin{array}{c}1.1628 \\
8\end{array}$ & $\begin{array}{c}0.009 \\
17\end{array}$ & $\begin{array}{c}0.0978 \\
0\end{array}$ & $\begin{array}{c}0.001 \\
09\end{array}$ & 1344 & 26 & 783 & 4 & 602 & 6 & $30.07 \%$ \\
\hline 13FR13\#65 & 56 & 519 & $\begin{array}{c}0.1 \\
1\end{array}$ & 0.05482 & $\begin{array}{c}0.000 \\
56\end{array}$ & $\begin{array}{c}0.6154 \\
4\end{array}$ & $\begin{array}{c}0.005 \\
98\end{array}$ & $\begin{array}{c}0.0814 \\
4\end{array}$ & $\begin{array}{c}0.000 \\
92\end{array}$ & 405 & 33 & 487 & 4 & 505 & 5 & $-3.56 \%$ \\
\hline 13FR13\#66 & 66 & 421 & $\begin{array}{c}0.1 \\
6\end{array}$ & 0.05420 & $\begin{array}{c}0.000 \\
63\end{array}$ & $\begin{array}{c}0.5732 \\
1\end{array}$ & $\begin{array}{c}0.006 \\
22\end{array}$ & $\begin{array}{c}0.0767 \\
3\end{array}$ & $\begin{array}{c}0.000 \\
88\end{array}$ & 379 & 35 & 460 & 4 & 477 & 5 & $-3.56 \%$ \\
\hline 13FR13\#67 & 79 & 543 & 0.1 & 0,10645 & $\begin{array}{c}0.001 \\
00\end{array}$ & $\begin{array}{c}0.8694 \\
3\end{array}$ & $\begin{array}{c}0.007 \\
41\end{array}$ & $\begin{array}{c}0.0592 \\
5\end{array}$ & $\begin{array}{c}0.000 \\
68\end{array}$ & 1739 & 26 & 635 & 4 & 371 & 4 & $71.16 \%$ \\
\hline 13FR13\#68 & 34 & 347 & $\begin{array}{c}0.1 \\
0\end{array}$ & 0.05791 & $\begin{array}{c}0.000 \\
89\end{array}$ & $\begin{array}{c}0.6631 \\
2\end{array}$ & $\begin{array}{c}0.009 \\
45\end{array}$ & $\begin{array}{c}0.0830 \\
8\end{array}$ & $\begin{array}{c}0.001 \\
04\end{array}$ & 526 & 41 & 517 & 6 & 514 & 6 & $0.58 \%$ \\
\hline 13FR13\#69 & 57 & 292 & $\begin{array}{c}0.2 \\
0\end{array}$ & 0.05632 & $\begin{array}{c}0.000 \\
82\end{array}$ & $\begin{array}{c}0.6255 \\
4\end{array}$ & $\begin{array}{c}0.008 \\
48\end{array}$ & $\begin{array}{c}0.0805 \\
8\end{array}$ & $\begin{array}{c}0.000 \\
99\end{array}$ & 465 & 40 & 493 & 5 & 500 & 6 & $-1.40 \%$ \\
\hline 13FR13\#70 & 171 & 528 & $\begin{array}{c}0.3 \\
2\end{array}$ & 0.05769 & $\begin{array}{c}0.000 \\
71\end{array}$ & $\begin{array}{c}0.6154 \\
2\end{array}$ & $\begin{array}{c}0.007 \\
09\end{array}$ & $\begin{array}{c}0.0773 \\
9\end{array}$ & $\begin{array}{c}0.000 \\
91\end{array}$ & 518 & 36 & 487 & 4 & 481 & 5 & $1.25 \%$ \\
\hline 13FR13\#71 & 60 & 89 & $\begin{array}{c}0.6 \\
7\end{array}$ & 0.05759 & $\begin{array}{c}0.001 \\
35\end{array}$ & $\begin{array}{c}0.6124 \\
1\end{array}$ & $\begin{array}{c}0.013 \\
28\end{array}$ & $\begin{array}{c}0.0771 \\
4\end{array}$ & $\begin{array}{c}0.001 \\
13\end{array}$ & 514 & 57 & 485 & 8 & 479 & 7 & $1.25 \%$ \\
\hline
\end{tabular}




\begin{tabular}{|c|c|c|c|c|c|c|c|c|c|c|c|c|c|c|c|c|}
\hline 13FR13\#72 & 162 & 530 & $\begin{array}{c}0.3 \\
1\end{array}$ & 0.05884 & $\begin{array}{c}0.000 \\
58\end{array}$ & $\begin{array}{c}0.6421 \\
9\end{array}$ & $\begin{array}{c}0.005 \\
99\end{array}$ & $\begin{array}{c}0.0791 \\
8\end{array}$ & $\begin{array}{c}0.000 \\
89\end{array}$ & 561 & 31 & 504 & 4 & 491 & 5 & $2.65 \%$ \\
\hline 13FR13\#73 & 61 & 311 & $\begin{array}{c}0.2 \\
0\end{array}$ & 0.05657 & $\begin{array}{c}0.000 \\
77\end{array}$ & $\begin{array}{c}0.6000 \\
0\end{array}$ & $\begin{array}{c}0.007 \\
57\end{array}$ & $\begin{array}{c}0.0769 \\
5\end{array}$ & $\begin{array}{c}0.000 \\
92\end{array}$ & 475 & 38 & 477 & 5 & 478 & 6 & $-0.21 \%$ \\
\hline 13FR13\#74 & 164 & 265 & $\begin{array}{c}0.6 \\
2\end{array}$ & 0.12474 & $\begin{array}{c}0.000 \\
78\end{array}$ & $\begin{array}{c}6.4023 \\
2\end{array}$ & $\begin{array}{c}0.040 \\
20\end{array}$ & $\begin{array}{c}0.3723 \\
6\end{array}$ & $\begin{array}{c}0.004 \\
07\end{array}$ & 2025 & 19 & 2033 & 6 & 2040 & $\begin{array}{l}1 \\
9\end{array}$ & $-0.74 \%$ \\
\hline 13FR13\#75 & 137 & 177 & $\begin{array}{c}0.7 \\
8\end{array}$ & 0.06209 & $\begin{array}{c}0.001 \\
09\end{array}$ & $\begin{array}{c}0.8843 \\
6\end{array}$ & $\begin{array}{c}0.014 \\
35\end{array}$ & $\begin{array}{c}0.1033 \\
3\end{array}$ & $\begin{array}{c}0.001 \\
37\end{array}$ & 677 & 43 & & 8 & 634 & 8 & $1.42 \%$ \\
\hline 13FR13\#76 & 75 & 609 & $\begin{array}{c}0.1 \\
2\end{array}$ & 0.05705 & $\begin{array}{c}0.000 \\
74\end{array}$ & $\begin{array}{c}0.6172 \\
3\end{array}$ & $\begin{array}{c}0.007 \\
48\end{array}$ & $\begin{array}{c}0.0785 \\
0\end{array}$ & $\begin{array}{c}0.000 \\
93\end{array}$ & & & & 5 & 487 & 6 & $0.21 \%$ \\
\hline 13FR13\#77 & 64 & 375 & $\begin{array}{c}0.1 \\
7\end{array}$ & 0.05771 & $\begin{array}{c}0.000 \\
90\end{array}$ & $\begin{array}{c}0.5992 \\
1\end{array}$ & $\begin{array}{c}0.008 \\
62\end{array}$ & $\begin{array}{c}0.0753 \\
3\end{array}$ & $\begin{array}{c}0.000 \\
94\end{array}$ & 519 & 41 & 477 & 5 & 468 & 6 & $1.92 \%$ \\
\hline 13FR13\#78 & 63 & 96 & $\begin{array}{c}0.6 \\
6\end{array}$ & 0.07539 & $\begin{array}{c}0.001 \\
29\end{array}$ & $\begin{array}{c}1.8060 \\
6\end{array}$ & $\begin{array}{c}0.028 \\
43\end{array}$ & $\begin{array}{c}0.1738 \\
1\end{array}$ & $\begin{array}{c}0.002 \\
40\end{array}$ & 1079 & 39 & 1048 & 10 & 1033 & $\begin{array}{l}1 \\
3\end{array}$ & $4.45 \%$ \\
\hline 13FR13\#79 & 79 & 304 & $\begin{array}{c}0.2 \\
6\end{array}$ & 0.05924 & $\begin{array}{c}0.000 \\
62\end{array}$ & $\begin{array}{c}0.8271 \\
5\end{array}$ & $\begin{array}{c}0.008 \\
13\end{array}$ & $\begin{array}{c}0.1013 \\
1\end{array}$ & $\begin{array}{c}0.001 \\
16\end{array}$ & 576 & 32 & 612 & 5 & 622 & 7 & $-1.61 \%$ \\
\hline 13FR13\#80 & 107 & 70 & $\begin{array}{c}1.5 \\
3\end{array}$ & 0.11205 & $\begin{array}{c}0.001 \\
35\end{array}$ & $\begin{array}{c}4.7725 \\
5\end{array}$ & $\begin{array}{c}0.053 \\
62\end{array}$ & $\begin{array}{c}0.3090 \\
2\end{array}$ & $\begin{array}{c}0.003 \\
99\end{array}$ & 1833 & 26 & 1780 & 9 & 1736 & 0 & $5.59 \%$ \\
\hline 13FR13\#81 & 167 & 454 & $\begin{array}{c}0.3 \\
7\end{array}$ & 0.05562 & $\begin{array}{c}0.000 \\
60\end{array}$ & $\begin{array}{c}0.5962 \\
6\end{array}$ & $\begin{array}{c}0.006 \\
05\end{array}$ & $\begin{array}{c}0.0777 \\
7\end{array}$ & $\begin{array}{c}0.000 \\
89\end{array}$ & 437 & 33 & 475 & 4 & 483 & 5 & $-1.66 \%$ \\
\hline 13FR13\#82 & 112 & 165 & $\begin{array}{c}0.6 \\
8\end{array}$ & 0.12920 & $\begin{array}{c}0.001 \\
01\end{array}$ & $\begin{array}{c}6.1666 \\
4\end{array}$ & $\begin{array}{c}0.046 \\
68\end{array}$ & $\begin{array}{c}0.3462 \\
7\end{array}$ & $\begin{array}{c}0.003 \\
97\end{array}$ & 2087 & 20 & 2000 & 7 & 1917 & ) & $8.87 \%$ \\
\hline 13FR13\#83 & 154 & 235 & $\begin{array}{c}0.6 \\
5\end{array}$ & 0.11175 & $\begin{array}{c}0.000 \\
84\end{array}$ & $\begin{array}{c}4.7687 \\
1\end{array}$ & $\begin{array}{c}0.034 \\
94\end{array}$ & $\begin{array}{c}0.3095 \\
9\end{array}$ & $\begin{array}{c}0.003 \\
49\end{array}$ & 1828 & 21 & 1779 & 6 & 1739 & 7 & $5.12 \%$ \\
\hline 13FR13\#84 & 26 & 131 & $\begin{array}{c}0.2 \\
0\end{array}$ & 0.056 & $\begin{array}{c}0.000 \\
97\end{array}$ & $\begin{array}{c}0.6722 \\
6\end{array}$ & $\begin{array}{c}0.010 \\
71\end{array}$ & $\begin{array}{c}0.0864 \\
7\end{array}$ & $\begin{array}{c}0.001 \\
12\end{array}$ & 468 & 45 & 522 & 7 & 535 & 7 & $-2.43 \%$ \\
\hline 13FR13\#85 & 59 & 492 & $\begin{array}{c}0.1 \\
2\end{array}$ & 0.05417 & $\begin{array}{c}0.000 \\
55\end{array}$ & $\begin{array}{c}0.5993 \\
9\end{array}$ & $\begin{array}{c}0.005 \\
81\end{array}$ & $\begin{array}{c}0.0802 \\
8\end{array}$ & $\begin{array}{c}0.000 \\
91\end{array}$ & 378 & 33 & 477 & 4 & 498 & 5 & $-4.22 \%$ \\
\hline 13FR13\#86 & 194 & 250 & $\begin{array}{c}0.7 \\
7\end{array}$ & 0.05600 & $\begin{array}{c}0.000 \\
89\end{array}$ & $\begin{array}{c}0.6095 \\
5\end{array}$ & $\begin{array}{c}0.009 \\
01\end{array}$ & $\begin{array}{c}0.0789 \\
7\end{array}$ & $\begin{array}{c}0.001 \\
00\end{array}$ & 452 & 42 & 483 & 6 & 490 & 6 & $-1.43 \%$ \\
\hline 13FR13\#87 & 260 & 407 & $\begin{array}{c}0.6 \\
4\end{array}$ & 0.14776 & $\begin{array}{c}0.002 \\
47\end{array}$ & $\begin{array}{c}8.3931 \\
5\end{array}$ & $\begin{array}{c}0.096 \\
74\end{array}$ & $\begin{array}{c}0.4119 \\
8\end{array}$ & $\begin{array}{c}0.004 \\
98\end{array}$ & 2320 & 29 & 2274 & 10 & 2224 & $\begin{array}{l}2 \\
3\end{array}$ & $4.32 \%$ \\
\hline 13FR13\#88 & 80 & 419 & $\begin{array}{c}0.1 \\
9\end{array}$ & 0.05727 & $\begin{array}{c}0.000 \\
75\end{array}$ & $\begin{array}{c}0.6263 \\
6\end{array}$ & $\begin{array}{c}0.007 \\
64\end{array}$ & $\begin{array}{c}0.0793 \\
5\end{array}$ & $\begin{array}{c}0.000 \\
95\end{array}$ & 502 & 37 & 494 & 5 & 492 & 6 & $0.41 \%$ \\
\hline 13FR13\#89 & 42 & 488 & 0.0 & 0.05534 & 0.000 & 0.6040 & 0.006 & 0.0791 & 0.000 & 426 & 35 & 480 & 4 & 491 & 5 & $-2.24 \%$ \\
\hline
\end{tabular}




\begin{tabular}{|c|c|c|c|c|c|c|c|c|c|c|c|c|c|c|c|c|}
\hline & & & 9 & & 64 & 2 & 58 & 9 & 92 & & & & & & & \\
\hline 13FR13\#90 & 48 & 83 & $\begin{array}{c}0.5 \\
8\end{array}$ & 0.05913 & $\begin{array}{c}0.002 \\
64\end{array}$ & $\begin{array}{c}0.7810 \\
2\end{array}$ & $\begin{array}{c}0.032 \\
44\end{array}$ & $\begin{array}{c}0.0958 \\
0\end{array}$ & $\begin{array}{c}0.001 \\
57\end{array}$ & 572 & $\begin{array}{c}10 \\
0\end{array}$ & 586 & 18 & 590 & 9 & $-0.68 \%$ \\
\hline \multicolumn{17}{|l|}{ 13FR18-1 } \\
\hline $\begin{array}{l}\text { 13FR18- } \\
1 \# 01\end{array}$ & 201 & 282 & $\begin{array}{c}0.7 \\
1\end{array}$ & 0.15735 & $\begin{array}{c}0.000 \\
92\end{array}$ & $\begin{array}{c}10.483 \\
56\end{array}$ & $\begin{array}{c}0.062 \\
70\end{array}$ & $\begin{array}{c}0.4832 \\
2\end{array}$ & $\begin{array}{c}0.005 \\
35\end{array}$ & 2427 & 11 & 2478 & 6 & 2541 & $\begin{array}{l}2 \\
3\end{array}$ & $-4.49 \%$ \\
\hline $\begin{array}{l}\text { 13FR18- } \\
1 \# 02\end{array}$ & 361 & 450 & $\begin{array}{c}0.8 \\
0\end{array}$ & 0.06213 & $\begin{array}{c}0.000 \\
57\end{array}$ & $\begin{array}{c}0.8889 \\
8\end{array}$ & $\begin{array}{c}0.007 \\
72\end{array}$ & $\begin{array}{c}0.1037 \\
6\end{array}$ & $\begin{array}{c}0.001 \\
18\end{array}$ & 679 & 11 & & 4 & 636 & 7 & $1.57 \%$ \\
\hline $\begin{array}{l}\text { 13FR18- } \\
1 \# 03\end{array}$ & 163 & 245 & $\begin{array}{c}0.6 \\
6\end{array}$ & 0.10995 & $\begin{array}{c}0.000 \\
74\end{array}$ & $\begin{array}{c}4.7686 \\
2\end{array}$ & $\begin{array}{c}0.031 \\
98\end{array}$ & $\begin{array}{c}0.3145 \\
4\end{array}$ & $\begin{array}{c}0.003 \\
53\end{array}$ & 17 & & 1779 & 6 & 1763 & $\begin{array}{l}1 \\
7\end{array}$ & $2.04 \%$ \\
\hline $\begin{array}{l}\text { 13FR18- } \\
1 \# 04\end{array}$ & 67 & 174 & $\begin{array}{c}0.3 \\
9\end{array}$ & 0.17198 & $\begin{array}{c}0.001 \\
08\end{array}$ & $\begin{array}{c}10.513 \\
76\end{array}$ & $\begin{array}{c}0.066 \\
59\end{array}$ & $\begin{array}{c}0.4434 \\
0\end{array}$ & $\begin{array}{c}0.004 \\
99\end{array}$ & 2577 & 11 & 2481 & 6 & 2366 & $\begin{array}{l}2 \\
2\end{array}$ & $8.92 \%$ \\
\hline $\begin{array}{l}\text { 13FR18- } \\
1 \# 05\end{array}$ & 93 & 81 & $\begin{array}{c}1.1 \\
5\end{array}$ & 0.06375 & $\begin{array}{c}0.001 \\
27\end{array}$ & $\begin{array}{c}0.8925 \\
2\end{array}$ & $\begin{array}{c}0.016 \\
31\end{array}$ & $\begin{array}{c}0.1015 \\
5\end{array}$ & $\begin{array}{c}0.001 \\
45\end{array}$ & 733 & 18 & 648 & 9 & 623 & 8 & $4.01 \%$ \\
\hline $\begin{array}{l}\text { 13FR18- } \\
1 \# 06\end{array}$ & 113 & 141 & $\begin{array}{c}0.8 \\
0\end{array}$ & 0.12098 & $\begin{array}{c}0.000 \\
92\end{array}$ & $\begin{array}{c}5.8950 \\
3\end{array}$ & $\begin{array}{c}0.043 \\
80\end{array}$ & $\begin{array}{c}0.3534 \\
2\end{array}$ & $\begin{array}{c}0.004 \\
07\end{array}$ & 1971 & 11 & 1961 & 6 & 1951 & $\begin{array}{l}1 \\
9\end{array}$ & $1.03 \%$ \\
\hline $\begin{array}{l}13 F R 18- \\
1 \# 07\end{array}$ & 69 & 41 & $\begin{array}{c}1.7 \\
1\end{array}$ & 0.11559 & $\begin{array}{c}0.001 \\
34\end{array}$ & $\begin{array}{c}6.2162 \\
4\end{array}$ & $\begin{array}{c}0.068 \\
33\end{array}$ & $\begin{array}{c}0.3900 \\
6\end{array}$ & $\begin{array}{c}0.005 \\
08\end{array}$ & 1889 & 11 & 2007 & 10 & 2123 & $\begin{array}{l}2 \\
4\end{array}$ & $-11.02 \%$ \\
\hline $\begin{array}{l}\text { 13FR18- } \\
1 \# 08\end{array}$ & 130 & 140 & $\begin{array}{c}0.9 \\
3\end{array}$ & 0.06035 & $\begin{array}{c}0.000 \\
92\end{array}$ & 0.8399 & $\begin{array}{c}0.011 \\
87\end{array}$ & $\begin{array}{c}0.1009 \\
4\end{array}$ & $\begin{array}{c}0.001 \\
29\end{array}$ & 616 & 14 & 619 & 7 & 620 & 8 & $-0.16 \%$ \\
\hline $\begin{array}{l}\text { 13FR18- } \\
1 \# 09\end{array}$ & 146 & 185 & $\begin{array}{c}0.7 \\
9\end{array}$ & 0.12171 & $\begin{array}{c}0.000 \\
85\end{array}$ & $\begin{array}{c}6.0036 \\
7\end{array}$ & $\begin{array}{c}0.041 \\
54\end{array}$ & $\begin{array}{c}0.3578 \\
0\end{array}$ & $\begin{array}{c}0.004 \\
06\end{array}$ & 1981 & 11 & 1976 & 6 & 1972 & $\begin{array}{l}1 \\
9\end{array}$ & $0.46 \%$ \\
\hline $\begin{array}{l}\text { 13FR18- } \\
1 \# 10\end{array}$ & 85 & 147 & $\begin{array}{c}0.5 \\
8\end{array}$ & 0.17218 & $\begin{array}{c}0.001 \\
14\end{array}$ & $\begin{array}{c}11.794 \\
54\end{array}$ & $\begin{array}{c}0.078 \\
63\end{array}$ & $\begin{array}{c}0.4968 \\
7\end{array}$ & $\begin{array}{c}0.005 \\
67\end{array}$ & 2579 & 10 & 2588 & 6 & 2600 & $\begin{array}{l}2 \\
4\end{array}$ & $-0.81 \%$ \\
\hline $\begin{array}{l}\text { 13FR18- } \\
1 \# 11\end{array}$ & 67 & 93 & $\begin{array}{c}0.7 \\
1\end{array}$ & 0.05783 & $\begin{array}{c}0.001 \\
12\end{array}$ & $\begin{array}{c}0.7875 \\
4\end{array}$ & $\begin{array}{c}0.014 \\
11\end{array}$ & $\begin{array}{c}0.0987 \\
8\end{array}$ & $\begin{array}{c}0.001 \\
37\end{array}$ & 523 & 18 & 590 & 8 & 607 & 8 & $-2.80 \%$ \\
\hline $\begin{array}{l}\text { 13FR18- } \\
1 \# 12\end{array}$ & 12 & 17 & $\begin{array}{c}0.7 \\
2\end{array}$ & 0.17223 & $\begin{array}{c}0.002 \\
36\end{array}$ & $\begin{array}{c}12.122 \\
60\end{array}$ & $\begin{array}{c}0.158 \\
82\end{array}$ & $\begin{array}{c}0.5105 \\
6\end{array}$ & $\begin{array}{c}0.007 \\
80\end{array}$ & 2579 & 11 & 2614 & 12 & 2659 & $\begin{array}{l}3 \\
3\end{array}$ & $-3.01 \%$ \\
\hline $\begin{array}{l}\text { 13FR18- } \\
1 \# 13\end{array}$ & 93 & 195 & $\begin{array}{c}0.4 \\
8\end{array}$ & 0.11951 & $\begin{array}{c}0.000 \\
83\end{array}$ & $\begin{array}{c}5.8375 \\
0\end{array}$ & $\begin{array}{c}0.040 \\
19\end{array}$ & $\begin{array}{c}0.3543 \\
1\end{array}$ & $\begin{array}{c}0.004 \\
01\end{array}$ & 1949 & 11 & 1952 & 6 & 1955 & $\begin{array}{l}1 \\
9\end{array}$ & $-0.31 \%$ \\
\hline $\begin{array}{l}\text { 13FR18- } \\
1 \# 14\end{array}$ & 69 & 228 & $\begin{array}{c}0.3 \\
0\end{array}$ & 0.05918 & $\begin{array}{c}0.000 \\
75\end{array}$ & $\begin{array}{c}0.7747 \\
5\end{array}$ & $\begin{array}{c}0.009 \\
19\end{array}$ & $\begin{array}{c}0.0949 \\
6\end{array}$ & $\begin{array}{c}0.001 \\
15\end{array}$ & 574 & 12 & 582 & 5 & 585 & 7 & $-0.51 \%$ \\
\hline $\begin{array}{l}\text { 13FR18- } \\
1 \# 15\end{array}$ & 82 & 154 & $\begin{array}{c}0.5 \\
3\end{array}$ & 0.10488 & $\begin{array}{c}0.000 \\
85\end{array}$ & $\begin{array}{c}4.3248 \\
9\end{array}$ & $\begin{array}{c}0.033 \\
95\end{array}$ & $\begin{array}{c}0.2991 \\
2\end{array}$ & $\begin{array}{c}0.003 \\
46\end{array}$ & 1712 & 11 & 1698 & 6 & 1687 & $\begin{array}{l}1 \\
7\end{array}$ & $1.48 \%$ \\
\hline 13FR18- & 166 & 207 & 0.8 & 0.05858 & 0.000 & 0.8280 & 0.009 & 0.1025 & 0.001 & 552 & 12 & 613 & 5 & 629 & 7 & $-2.54 \%$ \\
\hline
\end{tabular}




\begin{tabular}{|c|c|c|c|c|c|c|c|c|c|c|c|c|c|c|c|c|}
\hline $1 \# 16$ & & & 0 & & 74 & 1 & 75 & 3 & 24 & & & & & & & \\
\hline $\begin{array}{l}\text { 13FR18- } \\
1 \# 17\end{array}$ & 115 & 144 & $\begin{array}{c}0.8 \\
0\end{array}$ & 0.11106 & $\begin{array}{c}0.002 \\
71\end{array}$ & $\begin{array}{c}4.8588 \\
9\end{array}$ & $\begin{array}{c}0.101 \\
12\end{array}$ & $\begin{array}{c}0.3173 \\
1\end{array}$ & $\begin{array}{c}0.004 \\
06\end{array}$ & 1817 & 45 & 1795 & 18 & 1777 & $\begin{array}{l}2 \\
0\end{array}$ & $2.25 \%$ \\
\hline $\begin{array}{l}\text { 13FR18- } \\
1 \# 18\end{array}$ & 142 & 410 & $\begin{array}{c}0.3 \\
5\end{array}$ & 0.06231 & $\begin{array}{c}0.000 \\
60\end{array}$ & $\begin{array}{c}0.9981 \\
1\end{array}$ & $\begin{array}{c}0.009 \\
08\end{array}$ & $\begin{array}{c}0.1162 \\
1\end{array}$ & $\begin{array}{c}0.001 \\
33\end{array}$ & 685 & 11 & 703 & 5 & 709 & 8 & $-0.85 \%$ \\
\hline $\begin{array}{l}\text { 13FR18- } \\
1 \# 19\end{array}$ & 208 & 326 & $\begin{array}{c}0.6 \\
4\end{array}$ & 0.06430 & $\begin{array}{c}0.000 \\
64\end{array}$ & $\begin{array}{c}1.1160 \\
9\end{array}$ & $\begin{array}{c}0.010 \\
55\end{array}$ & $\begin{array}{c}0.1259 \\
2\end{array}$ & $\begin{array}{c}0.001 \\
46\end{array}$ & 752 & & & 5 & 765 & 8 & $-0.52 \%$ \\
\hline $\begin{array}{l}13 F R 18- \\
1 \# 20\end{array}$ & 74 & 98 & $\begin{array}{c}0.7 \\
5\end{array}$ & 0.16554 & $\begin{array}{c}0.003 \\
28\end{array}$ & $\begin{array}{c}9.2089 \\
8\end{array}$ & $\begin{array}{c}0.138 \\
07\end{array}$ & $\begin{array}{c}0.4034 \\
8\end{array}$ & $\begin{array}{c}0.005 \\
24\end{array}$ & & & & 14 & 2185 & $\begin{array}{l}2 \\
4\end{array}$ & $15.01 \%$ \\
\hline $\begin{array}{l}\text { 13FR18- } \\
1 \# 21\end{array}$ & 39 & 40 & $\begin{array}{c}0.9 \\
7\end{array}$ & 0.13466 & $\begin{array}{c}0.001 \\
59\end{array}$ & $\begin{array}{c}7.4279 \\
9\end{array}$ & $\begin{array}{c}0.082 \\
98\end{array}$ & $\begin{array}{c}0.4001 \\
9\end{array}$ & $\begin{array}{c}0.005 \\
39\end{array}$ & 2 & 11 & 2164 & 10 & 2170 & $\begin{array}{l}2 \\
5\end{array}$ & $-0.46 \%$ \\
\hline $\begin{array}{l}\text { 13FR18- } \\
1 \# 22\end{array}$ & 196 & 205 & $\begin{array}{c}0.9 \\
6\end{array}$ & 0.06552 & $\begin{array}{c}0.003 \\
00\end{array}$ & $\begin{array}{c}0.8306 \\
7\end{array}$ & $\begin{array}{c}0.036 \\
18\end{array}$ & $\begin{array}{c}0.0919 \\
5\end{array}$ & $\begin{array}{c}0.001 \\
31\end{array}$ & 791 & 99 & 614 & 20 & 567 & 8 & $8.29 \%$ \\
\hline $\begin{array}{l}\text { 13FR18- } \\
1 \# 23\end{array}$ & 197 & 372 & $\begin{array}{c}0.5 \\
3\end{array}$ & 0.05833 & $\begin{array}{c}0.000 \\
63\end{array}$ & $\begin{array}{c}0.7811 \\
0\end{array}$ & $\begin{array}{c}0.007 \\
97\end{array}$ & $\begin{array}{c}0.0971 \\
5\end{array}$ & $\begin{array}{c}0.001 \\
13\end{array}$ & 542 & 11 & 586 & 5 & 598 & 7 & $-2.01 \%$ \\
\hline $\begin{array}{l}\text { 13FR18- } \\
1 \# 24\end{array}$ & 47 & 68 & $\begin{array}{c}0.6 \\
9\end{array}$ & 0.06434 & $\begin{array}{c}0.001 \\
29\end{array}$ & $\begin{array}{c}0.9793 \\
4\end{array}$ & $\begin{array}{c}0.018 \\
11\end{array}$ & $\begin{array}{c}0.1104 \\
4\end{array}$ & $\begin{array}{c}0.001 \\
58\end{array}$ & 753 & 18 & 693 & 9 & 675 & 9 & $2.67 \%$ \\
\hline $\begin{array}{l}\text { 13FR18- } \\
1 \# 25\end{array}$ & 414 & 394 & $\begin{array}{c}1.0 \\
5\end{array}$ & 0.06087 & $\begin{array}{c}0.000 \\
65\end{array}$ & $\begin{array}{c}0.8574 \\
5\end{array}$ & $\begin{array}{c}0.008 \\
65\end{array}$ & $\begin{array}{c}0.1022 \\
0\end{array}$ & $\begin{array}{c}0.001 \\
19\end{array}$ & 635 & 11 & 629 & 5 & 627 & 7 & $0.32 \%$ \\
\hline $\begin{array}{l}\text { 13FR18- } \\
1 \# 26\end{array}$ & 148 & 205 & $\begin{array}{c}0.7 \\
2\end{array}$ & 0.06049 & $\begin{array}{c}0.000 \\
82\end{array}$ & 0.8418 & $\begin{array}{c}0.010 \\
61\end{array}$ & $\begin{array}{c}0.1009 \\
7\end{array}$ & $\begin{array}{c}0.001 \\
24\end{array}$ & 621 & 12 & 620 & 6 & 620 & 7 & $0.00 \%$ \\
\hline $\begin{array}{l}\text { 13FR18- } \\
1 \# 27\end{array}$ & 177 & 404 & $\begin{array}{c}0.4 \\
4\end{array}$ & 0.06012 & $\begin{array}{c}0.000 \\
62\end{array}$ & $\begin{array}{c}0.8455 \\
0\end{array}$ & $\begin{array}{c}0.008 \\
30\end{array}$ & $\begin{array}{c}0.1020 \\
3\end{array}$ & $\begin{array}{c}0.001 \\
19\end{array}$ & 608 & 11 & 622 & 5 & 626 & 7 & $-0.64 \%$ \\
\hline $\begin{array}{l}\text { 13FR18- } \\
1 \# 28\end{array}$ & 112 & 136 & $\begin{array}{c}0.8 \\
3\end{array}$ & 0.06 & $\begin{array}{c}0.000 \\
96\end{array}$ & $\begin{array}{c}0.9161 \\
2\end{array}$ & $\begin{array}{c}0.013 \\
18\end{array}$ & $\begin{array}{c}0.1078 \\
5\end{array}$ & $\begin{array}{c}0.001 \\
39\end{array}$ & 661 & 14 & 660 & 7 & 660 & 8 & $0.00 \%$ \\
\hline $\begin{array}{l}\text { 13FR18- } \\
1 \# 29\end{array}$ & 95 & 104 & $\begin{array}{c}0.9 \\
1\end{array}$ & 0.11430 & $\begin{array}{c}0.000 \\
98\end{array}$ & $\begin{array}{c}5.3019 \\
1\end{array}$ & $\begin{array}{c}0.044 \\
01\end{array}$ & $\begin{array}{c}0.3365 \\
7\end{array}$ & $\begin{array}{c}0.003 \\
98\end{array}$ & 1869 & 10 & 1869 & 7 & 1870 & $\begin{array}{l}1 \\
9\end{array}$ & $-0.05 \%$ \\
\hline $\begin{array}{l}\text { 13FR18- } \\
1 \# 30\end{array}$ & 61 & 79 & $\begin{array}{c}0.7 \\
7\end{array}$ & 0.21297 & $\begin{array}{c}0.001 \\
58\end{array}$ & $\begin{array}{c}17.879 \\
64\end{array}$ & $\begin{array}{c}0.134 \\
10\end{array}$ & $\begin{array}{c}0.6091 \\
5\end{array}$ & $\begin{array}{c}0.007 \\
30\end{array}$ & 2928 & 10 & 2983 & 7 & 3067 & $\begin{array}{l}2 \\
9\end{array}$ & $-4.53 \%$ \\
\hline $\begin{array}{l}\text { 13FR18- } \\
1 \# 31\end{array}$ & 87 & 107 & $\begin{array}{c}0.8 \\
1\end{array}$ & 0.05998 & $\begin{array}{c}0.001 \\
14\end{array}$ & $\begin{array}{c}0.9677 \\
8\end{array}$ & $\begin{array}{c}0.017 \\
02\end{array}$ & $\begin{array}{c}0.1170 \\
8\end{array}$ & $\begin{array}{c}0.001 \\
62\end{array}$ & 603 & 18 & 687 & 9 & 714 & 9 & $-3.78 \%$ \\
\hline $\begin{array}{l}\text { 13FR18- } \\
1 \# 32\end{array}$ & 701 & 536 & $\begin{array}{c}1.3 \\
1\end{array}$ & 0.06806 & $\begin{array}{c}0.000 \\
69\end{array}$ & $\begin{array}{c}0.7425 \\
7\end{array}$ & $\begin{array}{c}0.007 \\
08\end{array}$ & $\begin{array}{c}0.0791 \\
7\end{array}$ & $\begin{array}{c}0.000 \\
92\end{array}$ & 870 & 11 & 564 & 4 & 491 & 5 & $14.87 \%$ \\
\hline $\begin{array}{l}13 F R 18- \\
1 \# 33\end{array}$ & 87 & 179 & $\begin{array}{c}0.4 \\
8\end{array}$ & 0.11733 & $\begin{array}{c}0.002 \\
28\end{array}$ & $\begin{array}{c}4.6480 \\
1\end{array}$ & $\begin{array}{c}0.069 \\
74\end{array}$ & $\begin{array}{c}0.2873 \\
0\end{array}$ & $\begin{array}{c}0.003 \\
55\end{array}$ & 1916 & 36 & 1758 & 13 & 1628 & $\begin{array}{l}1 \\
8\end{array}$ & $17.69 \%$ \\
\hline
\end{tabular}




\begin{tabular}{|c|c|c|c|c|c|c|c|c|c|c|c|c|c|c|c|c|}
\hline $\begin{array}{l}\text { 13FR18- } \\
1 \# 34\end{array}$ & 164 & 197 & $\begin{array}{c}0.8 \\
3\end{array}$ & 0.05783 & $\begin{array}{c}0.000 \\
83\end{array}$ & $\begin{array}{c}0.7586 \\
1\end{array}$ & $\begin{array}{c}0.010 \\
19\end{array}$ & $\begin{array}{c}0.0951 \\
9\end{array}$ & $\begin{array}{c}0.001 \\
19\end{array}$ & 523 & 13 & 573 & 6 & 586 & 7 & $-2.22 \%$ \\
\hline $\begin{array}{l}\text { 13FR18- } \\
1 \# 35\end{array}$ & 219 & 226 & $\begin{array}{c}0.9 \\
7\end{array}$ & 0.05970 & $\begin{array}{c}0.000 \\
75\end{array}$ & $\begin{array}{c}0.8048 \\
4\end{array}$ & $\begin{array}{c}0.009 \\
48\end{array}$ & $\begin{array}{c}0.0978 \\
3\end{array}$ & $\begin{array}{c}0.001 \\
18\end{array}$ & 593 & 12 & 600 & 5 & 602 & 7 & $-0.33 \%$ \\
\hline $\begin{array}{l}\text { 13FR18- } \\
1 \# 36\end{array}$ & 46 & 93 & $\begin{array}{c}0.5 \\
0\end{array}$ & 0.06180 & $\begin{array}{c}0.001 \\
52\end{array}$ & $\begin{array}{c}0.8364 \\
6\end{array}$ & $\begin{array}{c}0.018 \\
86\end{array}$ & $\begin{array}{c}0.0982 \\
2\end{array}$ & $\begin{array}{c}0.001 \\
54\end{array}$ & 667 & 24 & 617 & 10 & 604 & 9 & $2.15 \%$ \\
\hline $\begin{array}{l}\text { 13FR18- } \\
1 \# 37\end{array}$ & 261 & 212 & $\begin{array}{c}1.2 \\
3\end{array}$ & 0.06055 & $\begin{array}{c}0.000 \\
83\end{array}$ & $\begin{array}{c}0.8476 \\
0\end{array}$ & $\begin{array}{c}0.010 \\
87\end{array}$ & $\begin{array}{c}0.1015 \\
8\end{array}$ & $\begin{array}{c}0.001 \\
26\end{array}$ & 623 & 12 & 623 & 6 & 624 & 7 & $-0.16 \%$ \\
\hline $\begin{array}{l}\text { 13FR18- } \\
1 \# 38\end{array}$ & 273 & 352 & $\begin{array}{c}0.7 \\
8\end{array}$ & 0.05856 & $\begin{array}{c}0.000 \\
90\end{array}$ & $\begin{array}{c}0.7572 \\
0\end{array}$ & $\begin{array}{c}0.010 \\
80\end{array}$ & $\begin{array}{c}0.0938 \\
3\end{array}$ & $\begin{array}{c}0.001 \\
20\end{array}$ & & & 72 & 6 & 578 & 7 & $-1.04 \%$ \\
\hline $\begin{array}{l}\text { 13FR18- } \\
1 \# 39\end{array}$ & 92 & 68 & $\begin{array}{c}1.3 \\
5\end{array}$ & 0.06271 & $\begin{array}{c}0.001 \\
58\end{array}$ & $\begin{array}{c}0.8894 \\
2\end{array}$ & $\begin{array}{c}0.020 \\
59\end{array}$ & $\begin{array}{c}0.1029 \\
2\end{array}$ & $\begin{array}{c}0.001 \\
65\end{array}$ & 698 & 24 & 646 & 11 & 631 & $\begin{array}{l}1 \\
0\end{array}$ & $2.38 \%$ \\
\hline $\begin{array}{l}\text { 13FR18- } \\
1 \# 40\end{array}$ & 532 & 570 & $\begin{array}{c}0.9 \\
3\end{array}$ & 0.08221 & $\begin{array}{c}0.001 \\
32\end{array}$ & $\begin{array}{c}0.5448 \\
8\end{array}$ & $\begin{array}{c}0.007 \\
84\end{array}$ & $\begin{array}{c}0.0481 \\
0\end{array}$ & $\begin{array}{c}0.000 \\
65\end{array}$ & 1251 & 13 & 442 & 5 & 303 & 4 & $45.87 \%$ \\
\hline $\begin{array}{l}\text { 13FR18- } \\
1 \# 41\end{array}$ & 41 & 49 & $\begin{array}{c}0.8 \\
4\end{array}$ & 0.11882 & $\begin{array}{c}0.001 \\
38\end{array}$ & $\begin{array}{c}5.6922 \\
2\end{array}$ & $\begin{array}{c}0.062 \\
22\end{array}$ & $\begin{array}{c}0.3476 \\
6\end{array}$ & $\begin{array}{c}0.004 \\
54\end{array}$ & 1939 & 11 & 1930 & 9 & 1923 & $\begin{array}{l}2 \\
2\end{array}$ & $0.83 \%$ \\
\hline $\begin{array}{l}\text { 13FR18- } \\
1 \# 42\end{array}$ & 70 & 149 & $\begin{array}{c}0.4 \\
7\end{array}$ & 0.07640 & $\begin{array}{c}0.001 \\
32\end{array}$ & $\begin{array}{c}1.0602 \\
2\end{array}$ & $\begin{array}{c}0.016 \\
56\end{array}$ & $\begin{array}{c}0.1007 \\
1\end{array}$ & $\begin{array}{c}0.001 \\
39\end{array}$ & 1106 & 14 & 734 & 8 & 619 & 8 & $18.58 \%$ \\
\hline $\begin{array}{l}\text { 13FR18- } \\
1 \# 43\end{array}$ & 133 & 208 & $\begin{array}{c}0.6 \\
4\end{array}$ & 0.11695 & $\begin{array}{c}0.001 \\
10\end{array}$ & 5.3388 & $\begin{array}{c}0.047 \\
81\end{array}$ & $\begin{array}{c}0.3313 \\
1\end{array}$ & $\begin{array}{c}0.004 \\
02\end{array}$ & 1910 & 10 & 1875 & 8 & 1845 & $\begin{array}{l}1 \\
9\end{array}$ & $3.52 \%$ \\
\hline $\begin{array}{l}\text { 13FR18- } \\
1 \# 44\end{array}$ & 118 & 215 & $\begin{array}{c}0.5 \\
5\end{array}$ & 0.05939 & $\begin{array}{c}0.001 \\
08\end{array}$ & $\begin{array}{c}0.8704 \\
1\end{array}$ & $\begin{array}{c}0.014 \\
63\end{array}$ & $\begin{array}{c}0.1063 \\
7\end{array}$ & $\begin{array}{c}0.001 \\
44\end{array}$ & 581 & 17 & 636 & 8 & 652 & 8 & $-2.45 \%$ \\
\hline $\begin{array}{l}\text { 13FR18- } \\
1 \# 45\end{array}$ & 24 & 41 & $\begin{array}{c}0.5 \\
8\end{array}$ & 0.13091 & $\begin{array}{c}0.001 \\
55\end{array}$ & $\begin{array}{c}7.7008 \\
8\end{array}$ & $\begin{array}{c}0.087 \\
01\end{array}$ & $\begin{array}{c}0.4269 \\
1\end{array}$ & $\begin{array}{c}0.005 \\
75\end{array}$ & 2110 & 11 & 2197 & 10 & 2292 & $\begin{array}{l}2 \\
6\end{array}$ & $-7.94 \%$ \\
\hline $\begin{array}{l}\text { 13FR18- } \\
1 \# 46\end{array}$ & 59 & 102 & $\begin{array}{c}0.5 \\
8\end{array}$ & 005796 & $\begin{array}{c}0.001 \\
13\end{array}$ & $\begin{array}{c}0.8488 \\
8\end{array}$ & $\begin{array}{c}0.015 \\
40\end{array}$ & $\begin{array}{c}0.1063 \\
0\end{array}$ & $\begin{array}{c}0.001 \\
47\end{array}$ & 528 & 19 & 624 & 8 & 651 & 9 & $-4.15 \%$ \\
\hline $\begin{array}{l}\text { 13FR18- } \\
1 \# 47\end{array}$ & 77 & 117 & $\begin{array}{c}0.6 \\
6\end{array}$ & 0.06087 & $\begin{array}{c}0.001 \\
12\end{array}$ & $\begin{array}{c}0.8093 \\
8\end{array}$ & $\begin{array}{c}0.013 \\
72\end{array}$ & $\begin{array}{c}0.0965 \\
0\end{array}$ & $\begin{array}{c}0.001 \\
32\end{array}$ & 635 & 17 & 602 & 8 & 594 & 8 & $1.35 \%$ \\
\hline $\begin{array}{l}\text { 13FR18- } \\
1 \# 48\end{array}$ & 122 & 118 & $\begin{array}{c}1.0 \\
3\end{array}$ & 0.06206 & $\begin{array}{c}0.000 \\
97\end{array}$ & $\begin{array}{c}0.9095 \\
7\end{array}$ & $\begin{array}{c}0.013 \\
14\end{array}$ & $\begin{array}{c}0.1063 \\
8\end{array}$ & $\begin{array}{c}0.001 \\
38\end{array}$ & 676 & 14 & 657 & 7 & 652 & 8 & $0.77 \%$ \\
\hline $\begin{array}{l}\text { 13FR18- } \\
1 \# 49\end{array}$ & 48 & 155 & $\begin{array}{c}0.3 \\
1\end{array}$ & 0.06033 & $\begin{array}{c}0.001 \\
04\end{array}$ & $\begin{array}{c}0.8612 \\
6\end{array}$ & $\begin{array}{c}0.013 \\
72\end{array}$ & $\begin{array}{c}0.1036 \\
1\end{array}$ & $\begin{array}{c}0.001 \\
38\end{array}$ & 615 & 16 & 631 & 7 & 636 & 8 & $-0.79 \%$ \\
\hline $\begin{array}{l}\text { 13FR18- } \\
1 \# 50\end{array}$ & 47 & 44 & $\begin{array}{c}1.0 \\
8\end{array}$ & 0.10702 & $\begin{array}{c}0.001 \\
45\end{array}$ & $\begin{array}{c}4.5805 \\
8\end{array}$ & $\begin{array}{c}0.057 \\
78\end{array}$ & $\begin{array}{c}0.3106 \\
4\end{array}$ & $\begin{array}{c}0.004 \\
24\end{array}$ & 1749 & 11 & 1746 & 11 & 1744 & $\begin{array}{l}2 \\
1\end{array}$ & $0.29 \%$ \\
\hline 13FR18- & 139 & 317 & 0.4 & 0.07132 & 0.000 & 1.5642 & 0.015 & 0.1592 & 0.001 & 967 & 11 & 956 & 6 & 952 & 1 & $0.42 \%$ \\
\hline
\end{tabular}




\begin{tabular}{|c|c|c|c|c|c|c|c|c|c|c|c|c|c|c|c|c|}
\hline $1 \# 51$ & & & 4 & & 73 & 6 & 26 & 0 & 88 & & & & & & 0 & \\
\hline $\begin{array}{l}\text { 13FR18- } \\
1 \# 52\end{array}$ & 98 & 72 & $\begin{array}{c}1.3 \\
7\end{array}$ & 0.06076 & $\begin{array}{c}0.001 \\
46\end{array}$ & $\begin{array}{c}0.8491 \\
5\end{array}$ & $\begin{array}{c}0.018 \\
82\end{array}$ & $\begin{array}{c}0.1014 \\
4\end{array}$ & $\begin{array}{c}0.001 \\
56\end{array}$ & 631 & 24 & 624 & 10 & 623 & 9 & $0.16 \%$ \\
\hline $\begin{array}{l}\text { 13FR18- } \\
1 \# 53\end{array}$ & 145 & 248 & $\begin{array}{c}0.5 \\
8\end{array}$ & 0.05707 & $\begin{array}{c}0.000 \\
78\end{array}$ & $\begin{array}{c}0.7641 \\
4\end{array}$ & $\begin{array}{c}0.009 \\
81\end{array}$ & $\begin{array}{c}0.0971 \\
8\end{array}$ & $\begin{array}{c}0.001 \\
20\end{array}$ & 494 & 13 & 576 & 6 & 598 & 7 & $-3.68 \%$ \\
\hline $\begin{array}{l}\text { 13FR18- } \\
1 \# 54\end{array}$ & 445 & 152 & $\begin{array}{c}2.9 \\
3\end{array}$ & 0.06092 & $\begin{array}{c}0.006 \\
28\end{array}$ & $\begin{array}{c}0.8799 \\
1\end{array}$ & $\begin{array}{c}0.089 \\
24\end{array}$ & $\begin{array}{c}0.1047 \\
5\end{array}$ & $\begin{array}{c}0.001 \\
92\end{array}$ & 636 & 23 & & 48 & 642 & $\begin{array}{l}1 \\
1\end{array}$ & $-0.16 \%$ \\
\hline $\begin{array}{l}\text { 13FR18- } \\
1 \# 55\end{array}$ & 296 & 359 & $\begin{array}{c}0.8 \\
3\end{array}$ & 0.11542 & $\begin{array}{c}0.000 \\
73\end{array}$ & $\begin{array}{c}5.4278 \\
4\end{array}$ & $\begin{array}{c}0.034 \\
61\end{array}$ & $\begin{array}{c}0.3413 \\
4\end{array}$ & $\begin{array}{c}0.003 \\
80\end{array}$ & & & & 5 & 1893 & $\begin{array}{l}1 \\
8\end{array}$ & $-0.37 \%$ \\
\hline $\begin{array}{l}\text { 13FR18- } \\
1 \# 56\end{array}$ & 87 & 103 & $\begin{array}{c}0.8 \\
4\end{array}$ & 0.05996 & $\begin{array}{c}0.001 \\
16\end{array}$ & $\begin{array}{c}0.7868 \\
2\end{array}$ & $\begin{array}{c}0.014 \\
03\end{array}$ & $\begin{array}{c}0.0952 \\
6\end{array}$ & $\begin{array}{c}0.001 \\
32\end{array}$ & 602 & 18 & 589 & 8 & 587 & 8 & $0.34 \%$ \\
\hline $\begin{array}{l}\text { 13FR18- } \\
1 \# 57\end{array}$ & 81 & 155 & $\begin{array}{c}0.5 \\
2\end{array}$ & 0.11224 & $\begin{array}{c}0.000 \\
87\end{array}$ & $\begin{array}{c}5.1558 \\
0\end{array}$ & $\begin{array}{c}0.039 \\
02\end{array}$ & $\begin{array}{c}0.3334 \\
3\end{array}$ & $\begin{array}{c}0.003 \\
85\end{array}$ & 836 & 11 & 1845 & 6 & 1855 & $\begin{array}{l}1 \\
9\end{array}$ & $-1.02 \%$ \\
\hline $\begin{array}{l}\text { 13FR18- } \\
1 \# 58\end{array}$ & 180 & 356 & $\begin{array}{c}0.5 \\
1\end{array}$ & 0.05803 & $\begin{array}{c}0.000 \\
60\end{array}$ & $\begin{array}{c}0.7688 \\
6\end{array}$ & $\begin{array}{c}0.007 \\
62\end{array}$ & $\begin{array}{c}0.0961 \\
7\end{array}$ & $\begin{array}{c}0.001 \\
12\end{array}$ & 531 & 11 & 579 & 4 & 592 & 7 & $-2.20 \%$ \\
\hline $\begin{array}{l}\text { 13FR18- } \\
1 \# 59\end{array}$ & 115 & 121 & $\begin{array}{c}0.9 \\
5\end{array}$ & 0.06870 & $\begin{array}{c}0.001 \\
04\end{array}$ & $\begin{array}{c}1.3043 \\
9\end{array}$ & $\begin{array}{c}0.018 \\
38\end{array}$ & $\begin{array}{c}0.1378 \\
2\end{array}$ & $\begin{array}{c}0.001 \\
80\end{array}$ & 890 & 13 & 848 & 8 & 832 & $\begin{array}{l}1 \\
0\end{array}$ & $1.92 \%$ \\
\hline $\begin{array}{l}\text { 13FR18- } \\
1 \# 60\end{array}$ & 86 & 95 & $\begin{array}{c}0.9 \\
1\end{array}$ & 0.06092 & $\begin{array}{c}0.001 \\
34\end{array}$ & $\begin{array}{c}0.8427 \\
0\end{array}$ & $\begin{array}{c}0.017 \\
00\end{array}$ & $\begin{array}{c}0.1004 \\
1\end{array}$ & $\begin{array}{c}0.001 \\
48\end{array}$ & 636 & 21 & 621 & 9 & 617 & 9 & $0.65 \%$ \\
\hline $\begin{array}{l}\text { 13FR18- } \\
1 \# 61\end{array}$ & 86 & 293 & $\begin{array}{c}0.2 \\
9\end{array}$ & 0.11704 & $\begin{array}{c}0.000 \\
77\end{array}$ & $\begin{array}{c}5.5237 \\
7\end{array}$ & $\begin{array}{c}0.036 \\
32\end{array}$ & $\begin{array}{c}0.3425 \\
9\end{array}$ & $\begin{array}{c}0.003 \\
84\end{array}$ & 1912 & 11 & 1904 & 6 & 1899 & $\begin{array}{l}1 \\
8\end{array}$ & $0.68 \%$ \\
\hline $\begin{array}{l}\text { 13FR18- } \\
1 \# 62\end{array}$ & 55 & 93 & $\begin{array}{c}0.6 \\
0\end{array}$ & 0.11962 & $\begin{array}{c}0.001 \\
25\end{array}$ & $\begin{array}{c}5.7360 \\
8\end{array}$ & $\begin{array}{c}0.057 \\
00\end{array}$ & $\begin{array}{c}0.3480 \\
9\end{array}$ & $\begin{array}{c}0.004 \\
38\end{array}$ & 1951 & 10 & 1937 & 9 & 1925 & $\begin{array}{l}2 \\
1\end{array}$ & $1.35 \%$ \\
\hline $\begin{array}{l}\text { 13FR18- } \\
1 \# 63\end{array}$ & 138 & 297 & $\begin{array}{c}0.4 \\
6\end{array}$ & 0 . & $\begin{array}{c}0.001 \\
87\end{array}$ & $\begin{array}{c}4.5510 \\
1\end{array}$ & $\begin{array}{c}0.058 \\
11\end{array}$ & $\begin{array}{c}0.3052 \\
1\end{array}$ & $\begin{array}{c}0.003 \\
54\end{array}$ & 1768 & 32 & 1740 & 11 & 1717 & $\begin{array}{l}1 \\
7\end{array}$ & $2.97 \%$ \\
\hline $\begin{array}{l}\text { 13FR18- } \\
1 \# 64\end{array}$ & 36 & 59 & $\begin{array}{c}0.6 \\
1\end{array}$ & 0. & $\begin{array}{c}0.001 \\
11\end{array}$ & $\begin{array}{c}4.9767 \\
6\end{array}$ & $\begin{array}{c}0.047 \\
60\end{array}$ & $\begin{array}{c}0.3271 \\
9\end{array}$ & $\begin{array}{c}0.004 \\
02\end{array}$ & 1806 & 10 & 1815 & 8 & 1825 & $\begin{array}{l}2 \\
0\end{array}$ & $-1.04 \%$ \\
\hline $\begin{array}{l}\text { 13FR18- } \\
1 \# 65\end{array}$ & 182 & 169 & $\begin{array}{c}1.0 \\
8\end{array}$ & 0.07097 & $\begin{array}{c}0.000 \\
75\end{array}$ & $\begin{array}{c}1.5870 \\
6\end{array}$ & $\begin{array}{c}0.015 \\
81\end{array}$ & $\begin{array}{c}0.1623 \\
3\end{array}$ & $\begin{array}{c}0.001 \\
92\end{array}$ & 957 & 11 & 965 & 6 & 970 & $\begin{array}{l}1 \\
1\end{array}$ & $-0.52 \%$ \\
\hline $\begin{array}{l}\text { 13FR18- } \\
1 \# 66\end{array}$ & 252 & 385 & $\begin{array}{c}0.6 \\
5\end{array}$ & 0.05936 & $\begin{array}{c}0.000 \\
57\end{array}$ & $\begin{array}{c}0.8733 \\
7\end{array}$ & $\begin{array}{c}0.008 \\
01\end{array}$ & $\begin{array}{c}0.1068 \\
1\end{array}$ & $\begin{array}{c}0.001 \\
23\end{array}$ & 580 & 11 & 637 & 4 & 654 & 7 & $-2.60 \%$ \\
\hline $\begin{array}{l}\text { 13FR18- } \\
1 \# 67\end{array}$ & 53 & 166 & $\begin{array}{c}0.3 \\
2\end{array}$ & 0.05961 & $\begin{array}{c}0.001 \\
63\end{array}$ & $\begin{array}{c}0.8388 \\
7\end{array}$ & $\begin{array}{c}0.020 \\
30\end{array}$ & $\begin{array}{c}0.1020 \\
7\end{array}$ & $\begin{array}{c}0.001 \\
30\end{array}$ & 589 & 61 & 619 & 11 & 627 & 8 & $-1.28 \%$ \\
\hline $\begin{array}{l}\text { 13FR18- } \\
1 \# 68\end{array}$ & 97 & 147 & $\begin{array}{c}0.6 \\
6\end{array}$ & 0.11535 & $\begin{array}{c}0.001 \\
03\end{array}$ & $\begin{array}{c}5.4673 \\
1\end{array}$ & $\begin{array}{c}0.046 \\
89\end{array}$ & $\begin{array}{c}0.3440 \\
7\end{array}$ & $\begin{array}{c}0.004 \\
11\end{array}$ & 1885 & 10 & 1895 & 7 & 1906 & $\begin{array}{l}2 \\
0\end{array}$ & $-1.10 \%$ \\
\hline
\end{tabular}




\begin{tabular}{|c|c|c|c|c|c|c|c|c|c|c|c|c|c|c|c|c|}
\hline $\begin{array}{l}\text { 13FR18- } \\
1 \# 69\end{array}$ & 119 & 132 & $\begin{array}{c}0.9 \\
0\end{array}$ & 0.06109 & $\begin{array}{c}0.000 \\
93\end{array}$ & $\begin{array}{c}0.8474 \\
5\end{array}$ & $\begin{array}{c}0.011 \\
94\end{array}$ & $\begin{array}{c}0.1007 \\
1\end{array}$ & $\begin{array}{c}0.001 \\
29\end{array}$ & 642 & 14 & 623 & 7 & 619 & 8 & $0.65 \%$ \\
\hline $\begin{array}{l}\text { 13FR18- } \\
1 \# 70\end{array}$ & 230 & 255 & $\begin{array}{c}0.9 \\
0\end{array}$ & 0.06165 & $\begin{array}{c}0.001 \\
09\end{array}$ & $\begin{array}{c}0.8462 \\
9\end{array}$ & $\begin{array}{c}0.013 \\
75\end{array}$ & $\begin{array}{c}0.0996 \\
6\end{array}$ & $\begin{array}{c}0.001 \\
35\end{array}$ & 662 & 16 & 623 & 8 & 612 & 8 & $1.80 \%$ \\
\hline $\begin{array}{l}\text { 13FR18- } \\
\text { 1\#71 }\end{array}$ & 88 & 159 & $\begin{array}{c}0.5 \\
5\end{array}$ & 0.05981 & $\begin{array}{c}0.000 \\
89\end{array}$ & $\begin{array}{c}0.8034 \\
7\end{array}$ & $\begin{array}{c}0.011 \\
19\end{array}$ & $\begin{array}{c}0.0975 \\
2\end{array}$ & $\begin{array}{c}0.001 \\
24\end{array}$ & 597 & 13 & 599 & 6 & 600 & 7 & $-0.17 \%$ \\
\hline $\begin{array}{l}\text { 13FR18- } \\
1 \# 72\end{array}$ & 106 & 226 & $\begin{array}{c}0.4 \\
7\end{array}$ & 0.08279 & $\begin{array}{c}0.000 \\
72\end{array}$ & $\begin{array}{c}2.4288 \\
1\end{array}$ & $\begin{array}{c}0.020 \\
30\end{array}$ & $\begin{array}{c}0.2129 \\
8\end{array}$ & $\begin{array}{c}0.002 \\
46\end{array}$ & 1264 & & 51 & 6 & 1245 & $\begin{array}{l}1 \\
3\end{array}$ & $1.53 \%$ \\
\hline $\begin{array}{l}\text { 13FR18- } \\
1 \# 73\end{array}$ & 38 & 67 & $\begin{array}{c}0.5 \\
6\end{array}$ & 0.07084 & $\begin{array}{c}0.001 \\
13\end{array}$ & $\begin{array}{c}1.4313 \\
9\end{array}$ & $\begin{array}{c}0.021 \\
17\end{array}$ & $\begin{array}{c}0.1467 \\
0\end{array}$ & $\begin{array}{c}0.001 \\
95\end{array}$ & & & 902 & 9 & 882 & $\begin{array}{l}1 \\
1\end{array}$ & $2.27 \%$ \\
\hline $\begin{array}{l}\text { 13FR18- } \\
1 \# 74\end{array}$ & 110 & 72 & $\begin{array}{c}1.5 \\
2\end{array}$ & 0.06231 & $\begin{array}{c}0.001 \\
47\end{array}$ & $\begin{array}{c}0.9106 \\
6\end{array}$ & $\begin{array}{c}0.019 \\
68\end{array}$ & $\begin{array}{c}0.1061 \\
1\end{array}$ & $\begin{array}{c}0.001 \\
64\end{array}$ & 685 & 22 & 657 & 10 & 650 & $\begin{array}{l}1 \\
0\end{array}$ & $1.08 \%$ \\
\hline $\begin{array}{l}\text { 13FR18- } \\
1 \# 75\end{array}$ & 159 & 53 & $\begin{array}{c}3.0 \\
1\end{array}$ & 0.12264 & $\begin{array}{c}0.001 \\
15\end{array}$ & $\begin{array}{c}6.1328 \\
4\end{array}$ & $\begin{array}{c}0.055 \\
33\end{array}$ & $\begin{array}{c}0.3630 \\
5\end{array}$ & $\begin{array}{c}0.004 \\
43\end{array}$ & 1995 & 10 & 1995 & 8 & 1997 & $\begin{array}{l}2 \\
1\end{array}$ & $-0.10 \%$ \\
\hline $\begin{array}{l}\text { 13FR18- } \\
1 \# 76\end{array}$ & 95 & 143 & $\begin{array}{c}0.6 \\
7\end{array}$ & 0.11908 & $\begin{array}{c}0.001 \\
01\end{array}$ & $\begin{array}{c}5.6966 \\
0\end{array}$ & $\begin{array}{c}0.046 \\
90\end{array}$ & $\begin{array}{c}0.3473 \\
2\end{array}$ & $\begin{array}{c}0.004 \\
11\end{array}$ & 1942 & 10 & 1931 & 7 & 1922 & $\begin{array}{l}2 \\
0\end{array}$ & $1.04 \%$ \\
\hline $\begin{array}{l}\text { 13FR18- } \\
1 \# 77\end{array}$ & 128 & 171 & $\begin{array}{c}0.7 \\
5\end{array}$ & 0.21004 & $\begin{array}{c}0.001 \\
59\end{array}$ & $\begin{array}{c}16.004 \\
78\end{array}$ & $\begin{array}{c}0.121 \\
73\end{array}$ & $\begin{array}{c}0.5532 \\
3\end{array}$ & $\begin{array}{c}0.006 \\
65\end{array}$ & 2906 & 10 & 2877 & 7 & 2839 & $\begin{array}{l}2 \\
8\end{array}$ & $2.36 \%$ \\
\hline $\begin{array}{l}\text { 13FR18- } \\
1 \# 78\end{array}$ & 43 & 315 & $\begin{array}{c}0.1 \\
4\end{array}$ & 0.12747 & $\begin{array}{c}0.000 \\
82\end{array}$ & 6.0657 & $\begin{array}{c}0.039 \\
27\end{array}$ & $\begin{array}{c}0.3454 \\
8\end{array}$ & $\begin{array}{c}0.003 \\
87\end{array}$ & 2063 & 11 & 1985 & 6 & 1913 & $\begin{array}{l}1 \\
9\end{array}$ & $7.84 \%$ \\
\hline $\begin{array}{l}\text { 13FR18- } \\
1 \# 79\end{array}$ & 133 & 497 & $\begin{array}{c}0.2 \\
7\end{array}$ & 0.11746 & $\begin{array}{c}0.000 \\
76\end{array}$ & $\begin{array}{c}4.8246 \\
3\end{array}$ & $\begin{array}{c}0.031 \\
43\end{array}$ & $\begin{array}{c}0.2982 \\
1\end{array}$ & $\begin{array}{c}0.003 \\
34\end{array}$ & 1918 & 11 & 1789 & 5 & 1682 & $\begin{array}{l}1 \\
7\end{array}$ & $14.03 \%$ \\
\hline $\begin{array}{l}\text { 13FR18- } \\
1 \# 80\end{array}$ & 64 & 69 & $\begin{array}{c}0.9 \\
3\end{array}$ & 0.11823 & $\begin{array}{c}0.001 \\
33\end{array}$ & $\begin{array}{c}5.6774 \\
7\end{array}$ & $\begin{array}{c}0.060 \\
29\end{array}$ & $\begin{array}{c}0.3486 \\
7\end{array}$ & $\begin{array}{c}0.004 \\
50\end{array}$ & 1930 & 10 & 1928 & 9 & 1928 & $\begin{array}{l}2 \\
2\end{array}$ & $0.10 \%$ \\
\hline $\begin{array}{l}\text { 13FR18- } \\
1 \# 81\end{array}$ & 88 & 187 & $\begin{array}{c}0.4 \\
7\end{array}$ & 0.17989 & $\begin{array}{c}0.001 \\
07\end{array}$ & $\begin{array}{c}12.617 \\
20\end{array}$ & $\begin{array}{c}0.077 \\
76\end{array}$ & $\begin{array}{c}0.5092 \\
5\end{array}$ & $\begin{array}{c}0.005 \\
70\end{array}$ & 2652 & 11 & 2652 & 6 & 2653 & $\begin{array}{l}2 \\
4\end{array}$ & $-0.04 \%$ \\
\hline $\begin{array}{l}\text { 13FR18- } \\
1 \# 82\end{array}$ & 209 & 324 & $\begin{array}{c}0.6 \\
5\end{array}$ & 0.05958 & $\begin{array}{c}0.000 \\
64\end{array}$ & $\begin{array}{c}0.8075 \\
6\end{array}$ & $\begin{array}{c}0.008 \\
19\end{array}$ & $\begin{array}{c}0.0984 \\
1\end{array}$ & $\begin{array}{c}0.001 \\
15\end{array}$ & 588 & 11 & 601 & 5 & 605 & 7 & $-0.66 \%$ \\
\hline $\begin{array}{l}\text { 13FR18- } \\
1 \# 83\end{array}$ & 148 & 347 & $\begin{array}{c}0.4 \\
3\end{array}$ & 0.05770 & $\begin{array}{c}0.000 \\
60\end{array}$ & $\begin{array}{c}0.7511 \\
0\end{array}$ & $\begin{array}{c}0.007 \\
45\end{array}$ & $\begin{array}{c}0.0945 \\
2\end{array}$ & $\begin{array}{c}0.001 \\
10\end{array}$ & 518 & 11 & 569 & 4 & 582 & 6 & $-2.23 \%$ \\
\hline $\begin{array}{l}\text { 13FR18- } \\
1 \# 84\end{array}$ & 109 & 106 & $\begin{array}{c}1.0 \\
2\end{array}$ & 0.06175 & $\begin{array}{c}0.001 \\
14\end{array}$ & $\begin{array}{c}0.8622 \\
8\end{array}$ & $\begin{array}{c}0.014 \\
73\end{array}$ & $\begin{array}{c}0.1013 \\
9\end{array}$ & $\begin{array}{c}0.001 \\
39\end{array}$ & 665 & 17 & 631 & 8 & 623 & 8 & $1.28 \%$ \\
\hline $\begin{array}{l}\text { 13FR18- } \\
1 \# 85\end{array}$ & 108 & 279 & $\begin{array}{c}0.3 \\
9\end{array}$ & 0.05983 & $\begin{array}{c}0.001 \\
51\end{array}$ & $\begin{array}{c}0.7532 \\
4\end{array}$ & $\begin{array}{c}0.016 \\
62\end{array}$ & $\begin{array}{c}0.0913 \\
1\end{array}$ & $\begin{array}{c}0.001 \\
11\end{array}$ & 597 & 56 & 570 & 10 & 563 & 7 & $1.24 \%$ \\
\hline 13FR18- & 338 & 269 & 1.2 & 0.11798 & 0.000 & 5.5169 & 0.041 & 0.3395 & 0.003 & 1926 & 11 & 1903 & 6 & 1884 & 1 & $2.23 \%$ \\
\hline
\end{tabular}




\begin{tabular}{|c|c|c|c|c|c|c|c|c|c|c|c|c|c|c|c|c|}
\hline $1 \# 86$ & & & 6 & & 90 & 7 & 18 & 3 & 92 & & & & & & 9 & \\
\hline $\begin{array}{l}\text { 13FR18- } \\
1 \# 87\end{array}$ & 60 & 101 & $\begin{array}{c}0.5 \\
9\end{array}$ & 0.11776 & $\begin{array}{c}0.001 \\
13\end{array}$ & $\begin{array}{c}5.5624 \\
8\end{array}$ & $\begin{array}{c}0.051 \\
00\end{array}$ & $\begin{array}{c}0.3429 \\
9\end{array}$ & $\begin{array}{c}0.004 \\
19\end{array}$ & 1923 & 10 & 1910 & 8 & 1901 & $\begin{array}{l}2 \\
0\end{array}$ & $1.16 \%$ \\
\hline $\begin{array}{l}\text { 13FR18- } \\
1 \# 88\end{array}$ & 94 & 215 & $\begin{array}{c}0.4 \\
4\end{array}$ & 0.06041 & $\begin{array}{c}0.000 \\
72\end{array}$ & $\begin{array}{c}0.8359 \\
4\end{array}$ & $\begin{array}{c}0.009 \\
39\end{array}$ & $\begin{array}{c}0.1004 \\
8\end{array}$ & $\begin{array}{c}0.001 \\
20\end{array}$ & 618 & 12 & 617 & 5 & 617 & 7 & $0.00 \%$ \\
\hline $\begin{array}{l}\text { 13FR18- } \\
1 \# 89\end{array}$ & 75 & 53 & $\begin{array}{c}1.4 \\
2\end{array}$ & 0.11587 & $\begin{array}{c}0.001 \\
54\end{array}$ & $\begin{array}{c}5.4541 \\
6\end{array}$ & $\begin{array}{c}0.068 \\
07\end{array}$ & $\begin{array}{c}0.3417 \\
9\end{array}$ & $\begin{array}{c}0.004 \\
71\end{array}$ & 1893 & 11 & & 11 & 1895 & $\begin{array}{l}2 \\
3\end{array}$ & $-0.11 \%$ \\
\hline $\begin{array}{l}\text { 13FR18- } \\
1 \# 90\end{array}$ & 47 & 336 & $\begin{array}{c}0.1 \\
4\end{array}$ & 0.10113 & $\begin{array}{c}0.000 \\
67\end{array}$ & $\begin{array}{c}4.0422 \\
6\end{array}$ & $\begin{array}{c}0.027 \\
03\end{array}$ & $\begin{array}{c}0.2902 \\
3\end{array}$ & $\begin{array}{c}0.003 \\
25\end{array}$ & 16 & & & 5 & 1643 & $\begin{array}{l}1 \\
6\end{array}$ & $0.12 \%$ \\
\hline $\begin{array}{l}\text { 13FR18- } \\
\text { 1\#91 }\end{array}$ & 149 & 178 & $\begin{array}{c}0.8 \\
4\end{array}$ & 0.07302 & $\begin{array}{c}0.000 \\
80\end{array}$ & $\begin{array}{c}1.6974 \\
8\end{array}$ & $\begin{array}{c}0.017 \\
53\end{array}$ & $\begin{array}{c}0.1688 \\
0\end{array}$ & $\begin{array}{c}0.002 \\
03\end{array}$ & 015 & 11 & 1008 & 7 & 1005 & $\begin{array}{l}1 \\
1\end{array}$ & $1.00 \%$ \\
\hline $\begin{array}{l}\text { 13FR18- } \\
\text { 1\#92 }\end{array}$ & 151 & 216 & $\begin{array}{c}0.7 \\
0\end{array}$ & 0.11484 & $\begin{array}{c}0.000 \\
97\end{array}$ & $\begin{array}{c}5.3428 \\
1\end{array}$ & $\begin{array}{c}0.043 \\
90\end{array}$ & $\begin{array}{c}0.3378 \\
2\end{array}$ & $\begin{array}{c}0.003 \\
99\end{array}$ & 1877 & 10 & 1876 & 7 & 1876 & $\begin{array}{l}1 \\
9\end{array}$ & $0.05 \%$ \\
\hline $\begin{array}{l}\text { 13FR18- } \\
1 \# 93\end{array}$ & 168 & 140 & $\begin{array}{c}1.2 \\
0\end{array}$ & 0.05877 & $\begin{array}{c}0.000 \\
93\end{array}$ & $\begin{array}{c}0.7705 \\
9\end{array}$ & $\begin{array}{c}0.011 \\
31\end{array}$ & $\begin{array}{c}0.0952 \\
1\end{array}$ & $\begin{array}{c}0.001 \\
22\end{array}$ & 559 & 14 & 580 & 6 & 586 & 7 & $-1.02 \%$ \\
\hline $\begin{array}{l}\text { 13FR18- } \\
\text { 1\#94 }\end{array}$ & 122 & 212 & $\begin{array}{c}0.5 \\
7\end{array}$ & 0.12307 & $\begin{array}{c}0.002 \\
69\end{array}$ & $\begin{array}{c}5.1608 \\
8\end{array}$ & $\begin{array}{c}0.091 \\
68\end{array}$ & $\begin{array}{c}0.3041 \\
4\end{array}$ & $\begin{array}{c}0.003 \\
88\end{array}$ & 2001 & 40 & 1846 & 15 & 1712 & $\begin{array}{l}1 \\
9\end{array}$ & $16.88 \%$ \\
\hline $\begin{array}{l}\text { 13FR18- } \\
\text { 1\#95 }\end{array}$ & 66 & 101 & $\begin{array}{c}0.6 \\
5\end{array}$ & 0.11066 & $\begin{array}{c}0.000 \\
96\end{array}$ & $\begin{array}{c}4.9308 \\
0\end{array}$ & $\begin{array}{c}0.041 \\
27\end{array}$ & $\begin{array}{c}0.3235 \\
7\end{array}$ & $\begin{array}{c}0.003 \\
83\end{array}$ & 1810 & 10 & 1808 & 7 & 1807 & $\begin{array}{l}1 \\
9\end{array}$ & $0.17 \%$ \\
\hline $\begin{array}{l}13 F R 18- \\
1 \# 96\end{array}$ & 409 & 493 & $\begin{array}{c}0.8 \\
3\end{array}$ & 0.10330 & $\begin{array}{c}0.000 \\
82\end{array}$ & $\begin{array}{c}1.4573 \\
4\end{array}$ & $\begin{array}{c}0.011 \\
00\end{array}$ & $\begin{array}{c}0.1024 \\
5\end{array}$ & $\begin{array}{c}0.001 \\
17\end{array}$ & 1684 & 11 & 913 & 5 & 629 & 7 & $45.15 \%$ \\
\hline $\begin{array}{l}\text { 13FR18- } \\
\text { 1\#97 }\end{array}$ & 84 & 38 & $\begin{array}{c}2.1 \\
9\end{array}$ & 0.118 & $\begin{array}{c}0.001 \\
57\end{array}$ & $\begin{array}{c}5.7287 \\
5\end{array}$ & $\begin{array}{c}0.070 \\
75\end{array}$ & $\begin{array}{c}0.3497 \\
8\end{array}$ & $\begin{array}{c}0.004 \\
81\end{array}$ & 1940 & 11 & 1936 & 11 & 1934 & $\begin{array}{l}2 \\
3\end{array}$ & $0.31 \%$ \\
\hline $\begin{array}{l}\text { 13FR18- } \\
\text { 1\#98 }\end{array}$ & 271 & 272 & $\begin{array}{c}1.0 \\
0\end{array}$ & 0.06003 & $\begin{array}{c}0.000 \\
73\end{array}$ & $\begin{array}{c}0.8018 \\
0\end{array}$ & $\begin{array}{c}0.009 \\
14\end{array}$ & $\begin{array}{c}0.0969 \\
9\end{array}$ & $\begin{array}{c}0.001 \\
16\end{array}$ & 605 & 12 & 598 & 5 & 597 & 7 & $0.17 \%$ \\
\hline $\begin{array}{l}\text { 13FR18- } \\
\text { 1\#99 }\end{array}$ & 60 & 63 & $\begin{array}{c}0.9 \\
6\end{array}$ & 0.06159 & $\begin{array}{c}0.001 \\
80\end{array}$ & $\begin{array}{c}0.7818 \\
3\end{array}$ & $\begin{array}{c}0.020 \\
88\end{array}$ & $\begin{array}{c}0.0921 \\
8\end{array}$ & $\begin{array}{c}0.001 \\
60\end{array}$ & 660 & 29 & 587 & 12 & 568 & 9 & $3.35 \%$ \\
\hline $\begin{array}{l}\text { 13FR18- } \\
\text { 1\#100 } \\
\text { 13FR19 }\end{array}$ & 192 & 168 & $\begin{array}{l}1.1 \\
4\end{array}$ & 0.05854 & $\begin{array}{c}0.000 \\
80\end{array}$ & $\begin{array}{c}0.7864 \\
6\end{array}$ & $\begin{array}{c}0.010 \\
06\end{array}$ & $\begin{array}{c}0.0975 \\
6\end{array}$ & $\begin{array}{c}0.001 \\
20\end{array}$ & 550 & 13 & 589 & 6 & 600 & 7 & $-1.83 \%$ \\
\hline 13FR19\#01 & 164 & 282 & $\begin{array}{c}0.5 \\
8\end{array}$ & 0.11587 & $\begin{array}{c}0.000 \\
89\end{array}$ & $\begin{array}{c}4.9118 \\
6\end{array}$ & $\begin{array}{c}0.036 \\
71\end{array}$ & $\begin{array}{c}0.3073 \\
6\end{array}$ & $\begin{array}{c}0.003 \\
50\end{array}$ & 1893 & 10 & 1804 & 6 & 1728 & $\begin{array}{l}1 \\
7\end{array}$ & $9.55 \%$ \\
\hline 13FR19\#02 & 136 & 230 & $\begin{array}{c}0.5 \\
9\end{array}$ & 0.12259 & $\begin{array}{c}0.000 \\
85\end{array}$ & $\begin{array}{c}5.3285 \\
5\end{array}$ & $\begin{array}{c}0.036 \\
49\end{array}$ & $\begin{array}{c}0.3151 \\
9\end{array}$ & $\begin{array}{c}0.003 \\
53\end{array}$ & 1994 & 11 & 1873 & 6 & 1766 & $\begin{array}{l}1 \\
7\end{array}$ & $12.91 \%$ \\
\hline 13FR19\#03 & 161 & 122 & 1.3 & 0.11557 & 0.001 & 3.9483 & 0.033 & 0.2477 & 0.002 & 1889 & 10 & 1624 & 7 & 1427 & 1 & $32.38 \%$ \\
\hline
\end{tabular}




\begin{tabular}{|c|c|c|c|c|c|c|c|c|c|c|c|c|c|c|c|c|}
\hline & & & 1 & & 05 & 2 & 73 & 4 & 92 & & & & & & 5 & \\
\hline 13FR19\#04 & 275 & 514 & $\begin{array}{c}0.5 \\
3\end{array}$ & 0.20453 & $\begin{array}{c}0.001 \\
09\end{array}$ & $\begin{array}{c}13.514 \\
02\end{array}$ & $\begin{array}{c}0.074 \\
85\end{array}$ & $\begin{array}{c}0.4791 \\
7\end{array}$ & $\begin{array}{c}0.005 \\
18\end{array}$ & 2863 & 10 & 2716 & 5 & 2524 & $\begin{array}{l}2 \\
3\end{array}$ & $13.43 \%$ \\
\hline 13FR19\#05 & 50 & 105 & $\begin{array}{c}0.4 \\
8\end{array}$ & 0.12700 & $\begin{array}{c}0.000 \\
96\end{array}$ & $\begin{array}{c}6.5830 \\
4\end{array}$ & $\begin{array}{c}0.048 \\
92\end{array}$ & $\begin{array}{c}0.3759 \\
3\end{array}$ & $\begin{array}{c}0.004 \\
30\end{array}$ & 2057 & 10 & 2057 & 7 & 2057 & $\begin{array}{l}2 \\
0\end{array}$ & $0.00 \%$ \\
\hline 13FR19\#06 & 73 & 134 & $\begin{array}{c}0.5 \\
4\end{array}$ & 0.11486 & $\begin{array}{c}0.000 \\
86\end{array}$ & $\begin{array}{c}5.3677 \\
6\end{array}$ & $\begin{array}{c}0.039 \\
21\end{array}$ & $\begin{array}{c}0.3389 \\
3\end{array}$ & $\begin{array}{c}0.003 \\
84\end{array}$ & 1878 & 11 & 18 & 6 & 1882 & $\begin{array}{l}1 \\
8\end{array}$ & $-0.21 \%$ \\
\hline 13FR19\#07 & 91 & 132 & $\begin{array}{c}0.6 \\
9\end{array}$ & 0.10910 & $\begin{array}{c}0.002 \\
36\end{array}$ & $\begin{array}{c}4.4754 \\
7\end{array}$ & $\begin{array}{c}0.079 \\
64\end{array}$ & $\begin{array}{c}0.2975 \\
2\end{array}$ & $\begin{array}{c}0.003 \\
67\end{array}$ & 1784 & & & 15 & 1679 & $\begin{array}{l}1 \\
8\end{array}$ & $6.25 \%$ \\
\hline 13FR19\#08 & 320 & 637 & $\begin{array}{c}0.5 \\
0\end{array}$ & 0.06385 & $\begin{array}{c}0.000 \\
55\end{array}$ & $\begin{array}{c}0.8096 \\
2\end{array}$ & $\begin{array}{c}0.006 \\
63\end{array}$ & $\begin{array}{c}0.0919 \\
8\end{array}$ & $\begin{array}{c}0.001 \\
03\end{array}$ & 737 & 11 & 602 & 4 & 567 & 6 & $6.17 \%$ \\
\hline 13FR19\#09 & 36 & 99 & $\begin{array}{c}0.3 \\
6\end{array}$ & 0.13298 & $\begin{array}{c}0.001 \\
02\end{array}$ & $\begin{array}{c}7.1024 \\
1\end{array}$ & $\begin{array}{c}0.053 \\
36\end{array}$ & $\begin{array}{c}0.3874 \\
3\end{array}$ & 0.004 & 2138 & 10 & 2124 & 7 & 2111 & $\begin{array}{l}2 \\
1\end{array}$ & $1.28 \%$ \\
\hline 13FR19\#10 & 96 & 346 & $\begin{array}{c}0.2 \\
8\end{array}$ & 0.18626 & $\begin{array}{c}0.001 \\
34\end{array}$ & $\begin{array}{c}13.405 \\
39\end{array}$ & $\begin{array}{c}0.095 \\
78\end{array}$ & $\begin{array}{c}0.5220 \\
7\end{array}$ & $\begin{array}{c}0.006 \\
05\end{array}$ & 2709 & 10 & 2709 & 7 & 2708 & $\begin{array}{l}2 \\
6\end{array}$ & $0.04 \%$ \\
\hline 13FR19\#11 & 133 & 242 & $\begin{array}{c}0.5 \\
5\end{array}$ & 0.05941 & $\begin{array}{c}0.000 \\
97\end{array}$ & $\begin{array}{c}0.7639 \\
2\end{array}$ & $\begin{array}{c}0.011 \\
55\end{array}$ & $\begin{array}{c}0.0932 \\
9\end{array}$ & $\begin{array}{c}0.001 \\
21\end{array}$ & 582 & 15 & 576 & 7 & 575 & 7 & $0.17 \%$ \\
\hline 13FR19\#12 & 255 & 342 & $\begin{array}{c}0.7 \\
5\end{array}$ & 0.06161 & $\begin{array}{c}0.000 \\
70\end{array}$ & $\begin{array}{c}0.8597 \\
6\end{array}$ & $\begin{array}{c}0.009 \\
14\end{array}$ & $\begin{array}{c}0.1012 \\
4\end{array}$ & $\begin{array}{c}0.001 \\
19\end{array}$ & 661 & 11 & 630 & 5 & 622 & 7 & $1.29 \%$ \\
\hline 13FR19\#13 & 467 & 807 & $\begin{array}{c}0.5 \\
8\end{array}$ & 0.08323 & $\begin{array}{c}0.002 \\
24\end{array}$ & $\begin{array}{c}0.8720 \\
1\end{array}$ & $\begin{array}{c}0.020 \\
68\end{array}$ & $\begin{array}{c}0.0759 \\
8\end{array}$ & $\begin{array}{c}0.000 \\
97\end{array}$ & 1275 & 54 & 637 & 11 & 472 & 6 & $34.96 \%$ \\
\hline 13FR19\#14 & 171 & 385 & $\begin{array}{c}0.4 \\
5\end{array}$ & 0.06188 & $\begin{array}{c}0.000 \\
67\end{array}$ & $\begin{array}{c}0.7800 \\
5\end{array}$ & $\begin{array}{c}0.007 \\
90\end{array}$ & $\begin{array}{c}0.0914 \\
6\end{array}$ & $\begin{array}{c}0.001 \\
06\end{array}$ & 670 & 11 & 586 & 5 & 564 & 6 & $3.90 \%$ \\
\hline 13FR19\#15 & 29 & 46 & $\begin{array}{c}0.6 \\
2\end{array}$ & 0.12303 & $\begin{array}{c}0.001 \\
97\end{array}$ & $\begin{array}{c}6.1928 \\
4\end{array}$ & $\begin{array}{c}0.092 \\
10\end{array}$ & $\begin{array}{c}0.3652 \\
0\end{array}$ & $\begin{array}{c}0.005 \\
59\end{array}$ & 2001 & 12 & 2003 & 13 & 2007 & $\begin{array}{l}2 \\
6\end{array}$ & $-0.30 \%$ \\
\hline 13FR19\#16 & 146 & 532 & $\begin{array}{c}0.2 \\
7\end{array}$ & 0.05857 & $\begin{array}{c}0.000 \\
70\end{array}$ & $\begin{array}{c}0.8079 \\
2\end{array}$ & $\begin{array}{c}0.009 \\
04\end{array}$ & $\begin{array}{c}0.1000 \\
9\end{array}$ & $\begin{array}{c}0.001 \\
18\end{array}$ & 551 & 12 & 601 & 5 & 615 & 7 & $-2.28 \%$ \\
\hline 13FR19\#17 & 192 & 649 & $\begin{array}{c}0.3 \\
0\end{array}$ & 0.15369 & $\begin{array}{c}0.000 \\
83\end{array}$ & $\begin{array}{c}6.7290 \\
9\end{array}$ & $\begin{array}{c}0.037 \\
16\end{array}$ & $\begin{array}{c}0.3176 \\
8\end{array}$ & $\begin{array}{c}0.003 \\
42\end{array}$ & 2387 & 11 & 2076 & 5 & 1778 & $\begin{array}{l}1 \\
7\end{array}$ & $34.25 \%$ \\
\hline 13FR19\#18 & 38 & 61 & $\begin{array}{c}0.6 \\
2\end{array}$ & 0.06398 & $\begin{array}{c}0.002 \\
01\end{array}$ & $\begin{array}{c}0.9298 \\
8\end{array}$ & $\begin{array}{c}0.026 \\
56\end{array}$ & $\begin{array}{c}0.1054 \\
6\end{array}$ & $\begin{array}{c}0.001 \\
97\end{array}$ & 741 & 31 & 668 & 14 & 646 & $\begin{array}{l}1 \\
1\end{array}$ & $3.41 \%$ \\
\hline 13FR19\#19 & 26 & 59 & $\begin{array}{c}0.4 \\
4\end{array}$ & 0.05520 & $\begin{array}{c}0.003 \\
43\end{array}$ & $\begin{array}{c}0.5004 \\
3\end{array}$ & $\begin{array}{c}0.029 \\
88\end{array}$ & $\begin{array}{c}0.0657 \\
5\end{array}$ & $\begin{array}{c}0.001 \\
13\end{array}$ & 420 & $\begin{array}{c}14 \\
3\end{array}$ & 412 & 20 & 411 & 7 & $0.24 \%$ \\
\hline 13FR19\#20 & 155 & 117 & $\begin{array}{c}1.3 \\
3\end{array}$ & 0.12792 & $\begin{array}{c}0.001 \\
03\end{array}$ & $\begin{array}{c}6.0967 \\
6\end{array}$ & $\begin{array}{c}0.046 \\
92\end{array}$ & $\begin{array}{c}0.3458 \\
2\end{array}$ & $\begin{array}{c}0.004 \\
00\end{array}$ & 2070 & 10 & 1990 & 7 & 1915 & $\begin{array}{l}1 \\
9\end{array}$ & $8.09 \%$ \\
\hline
\end{tabular}




\begin{tabular}{|c|c|c|c|c|c|c|c|c|c|c|c|c|c|c|c|c|}
\hline 13FR19\#21 & 237 & 514 & $\begin{array}{c}0.4 \\
6\end{array}$ & 0.05912 & $\begin{array}{c}0.000 \\
72\end{array}$ & $\begin{array}{c}0.8027 \\
7\end{array}$ & $\begin{array}{c}0.009 \\
06\end{array}$ & $\begin{array}{c}0.0985 \\
4\end{array}$ & $\begin{array}{c}0.001 \\
16\end{array}$ & 572 & 12 & 598 & 5 & 606 & 7 & $-1.32 \%$ \\
\hline 13FR19\#22 & 192 & 191 & $\begin{array}{c}1.0 \\
0\end{array}$ & 0.06000 & $\begin{array}{c}0.000 \\
77\end{array}$ & $\begin{array}{c}0.7880 \\
8\end{array}$ & $\begin{array}{c}0.009 \\
38\end{array}$ & $\begin{array}{c}0.0953 \\
2\end{array}$ & $\begin{array}{c}0.001 \\
14\end{array}$ & 604 & 12 & 590 & 5 & 587 & 7 & $0.51 \%$ \\
\hline 13FR19\#23 & 291 & 269 & $\begin{array}{c}1.0 \\
8\end{array}$ & 0.06101 & $\begin{array}{c}0.001 \\
25\end{array}$ & $\begin{array}{c}0.8823 \\
4\end{array}$ & $\begin{array}{c}0.016 \\
65\end{array}$ & $\begin{array}{c}0.1049 \\
4\end{array}$ & $\begin{array}{c}0.001 \\
50\end{array}$ & 640 & 19 & 642 & 9 & 643 & 9 & $-0.16 \%$ \\
\hline 13FR19\#24 & 40 & 239 & $\begin{array}{c}0.1 \\
7\end{array}$ & 0.12329 & $\begin{array}{c}0.001 \\
10\end{array}$ & $\begin{array}{c}6.4053 \\
3\end{array}$ & $\begin{array}{c}0.054 \\
76\end{array}$ & $\begin{array}{c}0.3770 \\
1\end{array}$ & $\begin{array}{c}0.004 \\
50\end{array}$ & 2004 & & 2033 & 8 & 2062 & $\begin{array}{l}2 \\
1\end{array}$ & $-2.81 \%$ \\
\hline 13FR19\#25 & 78 & 65 & $\begin{array}{c}1.2 \\
0\end{array}$ & 0.19077 & $\begin{array}{c}0.001 \\
96\end{array}$ & $\begin{array}{c}15.096 \\
90\end{array}$ & $\begin{array}{c}0.151 \\
91\end{array}$ & $\begin{array}{l}0.5742 \\
9\end{array}$ & $\begin{array}{c}0.007 \\
72\end{array}$ & 74 & & 282 & 10 & 2925 & $\begin{array}{l}3 \\
2\end{array}$ & $-6.02 \%$ \\
\hline 13FR19\#26 & 473 & 319 & $\begin{array}{c}1.4 \\
9\end{array}$ & 0.06281 & $\begin{array}{c}0.001 \\
20\end{array}$ & $\begin{array}{c}0.9954 \\
4\end{array}$ & $\begin{array}{c}0.017 \\
47\end{array}$ & $\begin{array}{c}0.1150 \\
0\end{array}$ & $\begin{array}{c}0.001 \\
61\end{array}$ & 702 & 17 & 701 & 9 & 702 & 9 & $-0.14 \%$ \\
\hline 13FR19\#27 & 171 & 316 & $\begin{array}{c}0.5 \\
4\end{array}$ & 0.05393 & $\begin{array}{c}0.000 \\
95\end{array}$ & $\begin{array}{c}0.7537 \\
4\end{array}$ & $\begin{array}{c}0.012 \\
31\end{array}$ & $\begin{array}{c}0.1014 \\
2\end{array}$ & $\begin{array}{c}0.001 \\
33\end{array}$ & 368 & 17 & 570 & 7 & 623 & 8 & $-8.51 \%$ \\
\hline 13FR19\#28 & 21 & 48 & $\begin{array}{c}0.4 \\
4\end{array}$ & 0.11485 & $\begin{array}{c}0.002 \\
21\end{array}$ & $\begin{array}{c}5.3974 \\
1\end{array}$ & $\begin{array}{c}0.095 \\
56\end{array}$ & $\begin{array}{c}0.3410 \\
3\end{array}$ & $\begin{array}{c}0.005 \\
73\end{array}$ & 1878 & 14 & 1884 & 15 & 1892 & 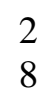 & $-0.74 \%$ \\
\hline 13FR19\#29 & 183 & 182 & $\begin{array}{c}1.0 \\
0\end{array}$ & 0.12901 & $\begin{array}{c}0.001 \\
15\end{array}$ & $\begin{array}{c}7.1977 \\
0\end{array}$ & $\begin{array}{c}0.061 \\
26\end{array}$ & $\begin{array}{c}0.4048 \\
6\end{array}$ & $\begin{array}{c}0.004 \\
85\end{array}$ & 2085 & 10 & 2136 & 8 & 2191 & $\begin{array}{l}2 \\
2\end{array}$ & $-4.84 \%$ \\
\hline 13FR19\#30 & 471 & 721 & $\begin{array}{c}0.6 \\
5\end{array}$ & 0.05933 & $\begin{array}{c}0.000 \\
72\end{array}$ & $\begin{array}{c}0.7633 \\
4\end{array}$ & $\begin{array}{c}0.008 \\
55\end{array}$ & $\begin{array}{c}0.0933 \\
7\end{array}$ & $\begin{array}{c}0.001 \\
10\end{array}$ & 579 & 11 & 576 & 5 & 575 & 6 & $0.17 \%$ \\
\hline 13FR19\#31 & 41 & 162 & $\begin{array}{c}0.2 \\
5\end{array}$ & 0.11294 & $\begin{array}{c}0.001 \\
38\end{array}$ & $\begin{array}{c}5.4873 \\
4\end{array}$ & $\begin{array}{c}0.062 \\
72\end{array}$ & $\begin{array}{c}0.3525 \\
8\end{array}$ & $\begin{array}{c}0.004 \\
66\end{array}$ & 1847 & 11 & 1899 & 10 & 1947 & 2 & $-5.14 \%$ \\
\hline 13FR19\#32 & 43 & 454 & $\begin{array}{c}0.1 \\
0\end{array}$ & 0.05964 & $\begin{array}{c}0.001 \\
00\end{array}$ & $\begin{array}{c}0.7928 \\
8\end{array}$ & $\begin{array}{c}0.012 \\
17\end{array}$ & $\begin{array}{c}0.0964 \\
7\end{array}$ & $\begin{array}{c}0.001 \\
26\end{array}$ & 591 & 15 & 593 & 7 & 594 & 7 & $-0.17 \%$ \\
\hline 13FR19\#33 & 229 & 423 & $\begin{array}{c}0.5 \\
4\end{array}$ & 0.05866 & $\begin{array}{c}0.000 \\
82\end{array}$ & $\begin{array}{c}0.8090 \\
3\end{array}$ & $\begin{array}{c}0.010 \\
46\end{array}$ & $\begin{array}{c}0.1000 \\
8\end{array}$ & $\begin{array}{c}0.001 \\
23\end{array}$ & 555 & 13 & 602 & 6 & 615 & 7 & $-2.11 \%$ \\
\hline 13FR19\#34 & 113 & 332 & $\begin{array}{c}0.3 \\
4\end{array}$ & 0.11256 & $\begin{array}{c}0.000 \\
83\end{array}$ & $\begin{array}{c}5.5573 \\
9\end{array}$ & $\begin{array}{c}0.039 \\
55\end{array}$ & $\begin{array}{c}0.3582 \\
8\end{array}$ & $\begin{array}{c}0.004 \\
04\end{array}$ & 1841 & 11 & 1910 & 6 & 1974 & $\begin{array}{l}1 \\
9\end{array}$ & $-6.74 \%$ \\
\hline 13FR19\#35 & 633 & 440 & $\begin{array}{c}1.4 \\
4\end{array}$ & 0.06082 & $\begin{array}{c}0.000 \\
92\end{array}$ & $\begin{array}{c}0.8632 \\
0\end{array}$ & $\begin{array}{c}0.011 \\
98\end{array}$ & $\begin{array}{c}0.1029 \\
9\end{array}$ & $\begin{array}{c}0.001 \\
30\end{array}$ & 633 & 13 & 632 & 7 & 632 & 8 & $0.00 \%$ \\
\hline 13FR19\#36 & 143 & 119 & $\begin{array}{c}1.2 \\
0\end{array}$ & 0.23841 & $\begin{array}{c}0.001 \\
60\end{array}$ & $\begin{array}{c}20.406 \\
76\end{array}$ & $\begin{array}{c}0.137 \\
55\end{array}$ & $\begin{array}{c}0.6211 \\
3\end{array}$ & $\begin{array}{c}0.007 \\
19\end{array}$ & 3109 & 10 & 3111 & 7 & 3114 & $\begin{array}{l}2 \\
9\end{array}$ & $-0.16 \%$ \\
\hline 13FR19\#37 & 40 & 428 & $\begin{array}{c}0.0 \\
9\end{array}$ & 0.16246 & $\begin{array}{c}0.001 \\
12\end{array}$ & $\begin{array}{c}10.236 \\
16\end{array}$ & $\begin{array}{c}0.068 \\
96\end{array}$ & $\begin{array}{c}0.4572 \\
1\end{array}$ & $\begin{array}{c}0.005 \\
19\end{array}$ & 2481 & 10 & 2456 & 6 & 2427 & $\begin{array}{l}2 \\
3\end{array}$ & $2.22 \%$ \\
\hline 13FR19\#38 & 66 & 100 & 0.6 & 0.05490 & 0.001 & 0.7026 & 0.022 & 0.0928 & 0.001 & 408 & 39 & 540 & 14 & 572 & 1 & $-5.59 \%$ \\
\hline
\end{tabular}




\begin{tabular}{|c|c|c|c|c|c|c|c|c|c|c|c|c|c|c|c|c|}
\hline & & & 6 & & 94 & 3 & 71 & 6 & 79 & & & & & & 1 & \\
\hline 13FR19\#39 & 307 & 339 & $\begin{array}{c}0.9 \\
1\end{array}$ & 0.05793 & $\begin{array}{c}0.000 \\
89\end{array}$ & $\begin{array}{c}0.9241 \\
2\end{array}$ & $\begin{array}{c}0.013 \\
05\end{array}$ & $\begin{array}{c}0.1157 \\
6\end{array}$ & $\begin{array}{c}0.001 \\
47\end{array}$ & 527 & 14 & 665 & 7 & 706 & 8 & $-5.81 \%$ \\
\hline 13FR19\#40 & 181 & 616 & $\begin{array}{c}0.2 \\
9\end{array}$ & 0.05827 & $\begin{array}{c}0.000 \\
71\end{array}$ & $\begin{array}{c}0.8321 \\
9\end{array}$ & $\begin{array}{c}0.009 \\
42\end{array}$ & $\begin{array}{c}0.1036 \\
3\end{array}$ & $\begin{array}{c}0.001 \\
23\end{array}$ & 540 & 12 & 615 & 5 & 636 & 7 & $-3.30 \%$ \\
\hline 13FR19\#41 & 42 & 79 & $\begin{array}{c}0.5 \\
3\end{array}$ & 0.06037 & $\begin{array}{c}0.004 \\
25\end{array}$ & $\begin{array}{c}0.5485 \\
9\end{array}$ & $\begin{array}{c}0.036 \\
98\end{array}$ & $\begin{array}{c}0.0659 \\
1\end{array}$ & $\begin{array}{c}0.001 \\
35\end{array}$ & 617 & 15 & & 24 & 411 & 8 & $8.03 \%$ \\
\hline 13FR19\#42 & 180 & 266 & $\begin{array}{c}0.6 \\
7\end{array}$ & 0.05923 & $\begin{array}{c}0.000 \\
97\end{array}$ & $\begin{array}{c}0.8467 \\
5\end{array}$ & $\begin{array}{c}0.012 \\
71\end{array}$ & $\begin{array}{c}0.1037 \\
3\end{array}$ & $\begin{array}{c}0.001 \\
35\end{array}$ & 576 & & & 7 & 636 & 8 & $-2.04 \%$ \\
\hline 13FR19\#43 & 52 & 93 & $\begin{array}{c}0.5 \\
6\end{array}$ & 0.09745 & $\begin{array}{c}0.001 \\
83\end{array}$ & $\begin{array}{c}3.4293 \\
6\end{array}$ & $\begin{array}{c}0.058 \\
48\end{array}$ & $\begin{array}{c}0.2553 \\
2\end{array}$ & $\begin{array}{c}0.004 \\
02\end{array}$ & 1576 & 14 & 1511 & 13 & 1466 & $\begin{array}{l}2 \\
1\end{array}$ & $7.50 \%$ \\
\hline $\begin{array}{c}\text { N13FR19\# } \\
01\end{array}$ & 238 & 366 & $\begin{array}{c}0.6 \\
5\end{array}$ & 0.08168 & $\begin{array}{c}0.001 \\
63\end{array}$ & $\begin{array}{c}0.9630 \\
5\end{array}$ & $\begin{array}{c}0.049 \\
56\end{array}$ & $\begin{array}{c}0.0851 \\
3\end{array}$ & $\begin{array}{c}0.001 \\
11\end{array}$ & 1238 & 38 & 685 & 26 & 527 & 7 & $30.04 \%$ \\
\hline $\begin{array}{c}\text { N13FR19\# } \\
02\end{array}$ & 1120 & 672 & $\begin{array}{c}1.6 \\
7\end{array}$ & 0.08144 & $\begin{array}{c}0.001 \\
13\end{array}$ & $\begin{array}{c}0.9401 \\
3\end{array}$ & $\begin{array}{c}0.034 \\
95\end{array}$ & $\begin{array}{c}0.0785 \\
2\end{array}$ & $\begin{array}{c}0.000 \\
83\end{array}$ & 1232 & 27 & 673 & 18 & 487 & 5 & $38.11 \%$ \\
\hline $\begin{array}{c}\text { N13FR19\# } \\
03\end{array}$ & 335 & 413 & $\begin{array}{c}0.8 \\
1\end{array}$ & 0.06219 & $\begin{array}{c}0.000 \\
93\end{array}$ & $\begin{array}{c}0.9511 \\
5\end{array}$ & $\begin{array}{c}0.039 \\
22\end{array}$ & $\begin{array}{c}0.1059 \\
0\end{array}$ & $\begin{array}{c}0.001 \\
12\end{array}$ & 681 & 32 & 679 & 20 & 649 & 7 & $4.59 \%$ \\
\hline $\begin{array}{c}\text { N13FR19\# } \\
04\end{array}$ & 913 & 965 & $\begin{array}{c}0.9 \\
5\end{array}$ & 0.13907 & $\begin{array}{c}0.001 \\
62\end{array}$ & $\begin{array}{c}1.3485 \\
8\end{array}$ & $\begin{array}{c}0.046 \\
85\end{array}$ & $\begin{array}{c}0.0628 \\
2\end{array}$ & $\begin{array}{c}0.000 \\
65\end{array}$ & 2216 & 20 & 867 & 20 & 393 & 4 & $120.70 \%$ \\
\hline $\begin{array}{c}\text { N13FR19\# } \\
05\end{array}$ & 394 & 377 & $\begin{array}{c}1.0 \\
4\end{array}$ & 0.09165 & $\begin{array}{c}0.001 \\
66\end{array}$ & $\begin{array}{c}1.1571 \\
1\end{array}$ & $\begin{array}{c}0.059 \\
19\end{array}$ & $\begin{array}{c}0.0922 \\
8\end{array}$ & $\begin{array}{c}0.001 \\
17\end{array}$ & 1460 & 34 & 781 & 28 & 569 & 7 & $37.19 \%$ \\
\hline $\begin{array}{c}\text { N13FR19\# } \\
06\end{array}$ & 228 & 276 & $\begin{array}{c}0.8 \\
3\end{array}$ & 0.09289 & $\begin{array}{c}0.001 \\
28\end{array}$ & $\begin{array}{c}1.7382 \\
6\end{array}$ & $\begin{array}{c}0.081 \\
43\end{array}$ & $\begin{array}{c}0.1411 \\
6\end{array}$ & $\begin{array}{c}0.001 \\
55\end{array}$ & 1486 & 26 & 1023 & 30 & 851 & 9 & $20.16 \%$ \\
\hline $\begin{array}{c}\text { N13FR19\# } \\
07\end{array}$ & 169 & 407 & $\begin{array}{c}0.4 \\
2\end{array}$ & 0.06426 & $\begin{array}{c}0.001 \\
13\end{array}$ & $\begin{array}{c}0.6861 \\
2\end{array}$ & $\begin{array}{c}0.030 \\
03\end{array}$ & $\begin{array}{c}0.0749 \\
2\end{array}$ & $\begin{array}{c}0.000 \\
86\end{array}$ & 750 & 37 & 531 & 18 & 466 & 5 & $13.91 \%$ \\
\hline $\begin{array}{c}\text { N13FR19\# } \\
08\end{array}$ & 248 & 789 & $\begin{array}{c}0.3 \\
1\end{array}$ & 0.09873 & $\begin{array}{c}0.001 \\
17\end{array}$ & $\begin{array}{c}1.1271 \\
0\end{array}$ & $\begin{array}{c}0.041 \\
33\end{array}$ & $\begin{array}{c}0.0763 \\
8\end{array}$ & $\begin{array}{c}0.000 \\
77\end{array}$ & 1600 & 22 & 766 & 20 & 475 & 5 & $61.52 \%$ \\
\hline $\begin{array}{c}\text { N13FR19\# } \\
09\end{array}$ & 172 & 682 & $\begin{array}{c}0.2 \\
5\end{array}$ & 0.06504 & $\begin{array}{c}0.000 \\
93\end{array}$ & $\begin{array}{c}0.8101 \\
6\end{array}$ & $\begin{array}{c}0.032 \\
70\end{array}$ & $\begin{array}{c}0.0852 \\
8\end{array}$ & $\begin{array}{c}0.000 \\
89\end{array}$ & 776 & 30 & 603 & 18 & 528 & 5 & $14.22 \%$ \\
\hline $\begin{array}{c}\text { N13FR19\# } \\
10\end{array}$ & 176 & 334 & $\begin{array}{c}0.5 \\
3\end{array}$ & 0.08437 & $\begin{array}{c}0.001 \\
65\end{array}$ & $\begin{array}{c}0.7661 \\
9\end{array}$ & $\begin{array}{c}0.038 \\
13\end{array}$ & $\begin{array}{c}0.0680 \\
7\end{array}$ & $\begin{array}{c}0.000 \\
88\end{array}$ & 1301 & 38 & 578 & 22 & 425 & 5 & $36.07 \%$ \\
\hline $\begin{array}{c}\text { N13FR19\# } \\
11\end{array}$ & 175 & 236 & $\begin{array}{c}0.7 \\
4\end{array}$ & 0.07342 & $\begin{array}{c}0.001 \\
18\end{array}$ & $\begin{array}{c}1.4044 \\
8\end{array}$ & $\begin{array}{c}0.074 \\
30\end{array}$ & $\begin{array}{c}0.1374 \\
0\end{array}$ & $\begin{array}{c}0.001 \\
58\end{array}$ & 1026 & 32 & 891 & 31 & 830 & 9 & $7.34 \%$ \\
\hline $\begin{array}{c}\text { N13FR19\# } \\
12\end{array}$ & 210 & 207 & $\begin{array}{c}1.0 \\
1\end{array}$ & 0.12646 & $\begin{array}{c}0.021 \\
04\end{array}$ & $\begin{array}{c}1.1750 \\
5\end{array}$ & $\begin{array}{c}0.506 \\
21\end{array}$ & $\begin{array}{c}0.1577 \\
0\end{array}$ & $\begin{array}{c}0.017 \\
52\end{array}$ & 2049 & $\begin{array}{c}26 \\
8\end{array}$ & 789 & $\begin{array}{c}23 \\
6\end{array}$ & 944 & $\begin{array}{l}9 \\
8\end{array}$ & $-16.42 \%$ \\
\hline
\end{tabular}




\begin{tabular}{|c|c|c|c|c|c|c|c|c|c|c|c|c|c|c|c|c|}
\hline $\begin{array}{c}\text { N13FR19\# } \\
13\end{array}$ & 253 & 695 & $\begin{array}{c}0.3 \\
6\end{array}$ & 0.06405 & $\begin{array}{c}0.000 \\
89\end{array}$ & $\begin{array}{c}0.7850 \\
9\end{array}$ & $\begin{array}{c}0.032 \\
71\end{array}$ & $\begin{array}{c}0.0831 \\
3\end{array}$ & $\begin{array}{c}0.000 \\
85\end{array}$ & 743 & 29 & 588 & 19 & 515 & 5 & $14.30 \%$ \\
\hline $\begin{array}{c}\text { N13FR19\# } \\
14\end{array}$ & 412 & 423 & $\begin{array}{c}0.9 \\
7\end{array}$ & 0.18964 & $\begin{array}{c}0.001 \\
47\end{array}$ & $\begin{array}{c}9.9468 \\
5\end{array}$ & $\begin{array}{c}0.481 \\
75\end{array}$ & $\begin{array}{c}0.3856 \\
7\end{array}$ & $\begin{array}{c}0.003 \\
66\end{array}$ & 2739 & 13 & 2430 & 45 & 2103 & $\begin{array}{l}1 \\
7\end{array}$ & $30.26 \%$ \\
\hline $\begin{array}{c}\text { N13FR19\# } \\
15\end{array}$ & 162 & 222 & $\begin{array}{c}0.7 \\
3\end{array}$ & 0.12633 & $\begin{array}{c}0.001 \\
21\end{array}$ & $\begin{array}{c}6.1543 \\
0\end{array}$ & $\begin{array}{c}0.338 \\
16\end{array}$ & $\begin{array}{c}0.3576 \\
5\end{array}$ & $\begin{array}{c}0.003 \\
62\end{array}$ & 2048 & 17 & 1998 & 48 & 1971 & $\begin{array}{l}1 \\
7\end{array}$ & $3.88 \%$ \\
\hline $\begin{array}{c}\text { N13FR19\# } \\
16\end{array}$ & 103 & 687 & $\begin{array}{c}0.1 \\
5\end{array}$ & 0.10668 & $\begin{array}{c}0.000 \\
78\end{array}$ & $\begin{array}{c}4.3426 \\
3\end{array}$ & $\begin{array}{c}0.175 \\
30\end{array}$ & $\begin{array}{c}0.2712 \\
2\end{array}$ & $\begin{array}{c}0.002 \\
38\end{array}$ & 1744 & & 70 & 33 & 1547 & $\begin{array}{l}1 \\
2\end{array}$ & $12.70 \%$ \\
\hline $\begin{array}{c}\text { N13FR19\# } \\
17\end{array}$ & 229 & 305 & $\begin{array}{c}0.7 \\
5\end{array}$ & 0.07381 & $\begin{array}{c}0.001 \\
00\end{array}$ & $\begin{array}{c}1.8197 \\
1\end{array}$ & $\begin{array}{c}0.097 \\
39\end{array}$ & $\begin{array}{c}0.1777 \\
0\end{array}$ & $\begin{array}{c}0.001 \\
90\end{array}$ & 1036 & & J5. & 35 & 1054 & $\begin{array}{l}1 \\
0\end{array}$ & $-1.73 \%$ \\
\hline $\begin{array}{c}\text { N13FR19\# } \\
18\end{array}$ & 639 & 1094 & $\begin{array}{c}0.5 \\
8\end{array}$ & 0.08694 & $\begin{array}{c}0.001 \\
10\end{array}$ & $\begin{array}{c}0.8012 \\
8\end{array}$ & $\begin{array}{c}0.034 \\
10\end{array}$ & $\begin{array}{c}0.0593 \\
6\end{array}$ & $\begin{array}{c}0.000 \\
61\end{array}$ & 1359 & 24 & 598 & 19 & 372 & 4 & $60.77 \%$ \\
\hline $\begin{array}{c}\text { N13FR19\# } \\
19\end{array}$ & 50 & 57 & $\begin{array}{c}0.8 \\
7\end{array}$ & 0.14706 & $\begin{array}{c}0.002 \\
27\end{array}$ & $\begin{array}{c}7.2712 \\
9\end{array}$ & $\begin{array}{c}0.679 \\
26\end{array}$ & $\begin{array}{c}0.4001 \\
7\end{array}$ & $\begin{array}{c}0.005 \\
73\end{array}$ & 2312 & 26 & 2145 & 83 & 2170 & 6 & $6.56 \%$ \\
\hline $\begin{array}{c}\text { N13FR19\# } \\
20\end{array}$ & 508 & 278 & $\begin{array}{c}1.8 \\
3\end{array}$ & 0.08653 & $\begin{array}{c}0.001 \\
41\end{array}$ & $\begin{array}{c}1.2407 \\
7\end{array}$ & $\begin{array}{c}0.069 \\
34\end{array}$ & $\begin{array}{c}0.1028 \\
0\end{array}$ & $\begin{array}{c}0.001 \\
22\end{array}$ & 1350 & 31 & 819 & 31 & 631 & 7 & $29.87 \%$ \\
\hline $\begin{array}{c}\text { N13FR19\# } \\
21\end{array}$ & 19 & 186 & $\begin{array}{c}0.1 \\
0\end{array}$ & 0.15700 & $\begin{array}{c}0.001 \\
46\end{array}$ & $\begin{array}{c}9.1085 \\
2\end{array}$ & $\begin{array}{c}0.577 \\
57\end{array}$ & $\begin{array}{c}0.4200 \\
9\end{array}$ & $\begin{array}{c}0.004 \\
34\end{array}$ & 2424 & 16 & 2349 & 58 & 2261 & 0 & $7.20 \%$ \\
\hline $\begin{array}{c}\text { N13FR19\# } \\
22\end{array}$ & 54 & 66 & $\begin{array}{c}0.8 \\
2\end{array}$ & 0.11705 & $\begin{array}{c}0.002 \\
12\end{array}$ & 4.7812 & $\begin{array}{c}0.459 \\
47\end{array}$ & $\begin{array}{c}0.2860 \\
9\end{array}$ & $\begin{array}{c}0.004 \\
20\end{array}$ & 1912 & 32 & 1782 & 81 & 1622 & $\begin{array}{l}2 \\
1\end{array}$ & $17.86 \%$ \\
\hline $\begin{array}{c}\text { N13FR19\# } \\
23\end{array}$ & 775 & 280 & $\begin{array}{c}2.7 \\
7\end{array}$ & 0.08916 & $\begin{array}{c}0.001 \\
71\end{array}$ & $\begin{array}{c}1.0232 \\
5\end{array}$ & $\begin{array}{c}0.063 \\
55\end{array}$ & $\begin{array}{c}0.0830 \\
1\end{array}$ & $\begin{array}{c}0.001 \\
09\end{array}$ & 1408 & 36 & 716 & 32 & 514 & 6 & $39.20 \%$ \\
\hline $\begin{array}{c}\text { N13FR19\# } \\
24\end{array}$ & 605 & 438 & $\begin{array}{c}1.3 \\
8\end{array}$ & 0.09248 & $\begin{array}{c}0.001 \\
07\end{array}$ & $\begin{array}{c}2.1699 \\
6\end{array}$ & $\begin{array}{c}0.120 \\
61\end{array}$ & $\begin{array}{c}0.1764 \\
6\end{array}$ & $\begin{array}{c}0.001 \\
80\end{array}$ & 1477 & 22 & 1172 & 39 & 1048 & $\begin{array}{l}1 \\
0\end{array}$ & $41.01 \%$ \\
\hline $\begin{array}{c}\text { N13FR19\# } \\
25\end{array}$ & 139 & 250 & $\begin{array}{c}0.5 \\
6\end{array}$ & 0.056 & $\begin{array}{c}0.001 \\
41\end{array}$ & $\begin{array}{c}0.5477 \\
7\end{array}$ & $\begin{array}{c}0.035 \\
91\end{array}$ & $\begin{array}{c}0.0683 \\
5\end{array}$ & $\begin{array}{c}0.000 \\
95\end{array}$ & 489 & 54 & 444 & 24 & 426 & 6 & $4.06 \%$ \\
\hline $\begin{array}{c}\text { N13FR19\# } \\
26\end{array}$ & 219 & 431 & $\begin{array}{c}0.5 \\
1\end{array}$ & 0.05819 & $\begin{array}{c}0.000 \\
96\end{array}$ & $\begin{array}{c}0.7759 \\
4\end{array}$ & $\begin{array}{c}0.044 \\
56\end{array}$ & $\begin{array}{c}0.0908 \\
2\end{array}$ & $\begin{array}{c}0.001 \\
00\end{array}$ & 536 & 36 & 583 & 25 & 560 & 6 & $4.07 \%$ \\
\hline \multicolumn{17}{|l|}{ 13FR21 } \\
\hline 13FR21\#01 & 41 & 201 & $\begin{array}{c}0.2 \\
0\end{array}$ & 0.06186 & $\begin{array}{c}0.001 \\
63\end{array}$ & $\begin{array}{c}0.9247 \\
4\end{array}$ & $\begin{array}{c}0.022 \\
28\end{array}$ & $\begin{array}{c}0.1083 \\
7\end{array}$ & $\begin{array}{c}0.001 \\
76\end{array}$ & 669 & 26 & 665 & 12 & 663 & $\begin{array}{l}1 \\
0\end{array}$ & $0.30 \%$ \\
\hline 13FR21\#02 & 44 & 91 & $\begin{array}{c}0.4 \\
8\end{array}$ & 0.16170 & $\begin{array}{c}0.002 \\
60\end{array}$ & $\begin{array}{c}10.016 \\
29\end{array}$ & $\begin{array}{c}0.150 \\
82\end{array}$ & $\begin{array}{c}0.4490 \\
3\end{array}$ & $\begin{array}{c}0.007 \\
29\end{array}$ & 2474 & 12 & 2436 & 14 & 2391 & $\begin{array}{l}3 \\
2\end{array}$ & $3.47 \%$ \\
\hline 13FR21\#03 & 31 & 315 & $\begin{array}{c}0.1 \\
0\end{array}$ & 0.05924 & $\begin{array}{c}0.001 \\
31\end{array}$ & $\begin{array}{c}0.7801 \\
6\end{array}$ & $\begin{array}{c}0.015 \\
86\end{array}$ & $\begin{array}{c}0.0954 \\
6\end{array}$ & $\begin{array}{c}0.001 \\
39\end{array}$ & 576 & 21 & 586 & 9 & 588 & 8 & $-0.34 \%$ \\
\hline
\end{tabular}




\begin{tabular}{|c|c|c|c|c|c|c|c|c|c|c|c|c|c|c|c|c|}
\hline 13FR21\#04 & 41 & 88 & $\begin{array}{c}0.4 \\
7\end{array}$ & 0.18202 & $\begin{array}{c}0.002 \\
61\end{array}$ & $\begin{array}{c}12.391 \\
74\end{array}$ & $\begin{array}{c}0.168 \\
42\end{array}$ & $\begin{array}{c}0.4935 \\
1\end{array}$ & $\begin{array}{c}0.007 \\
68\end{array}$ & 2671 & 12 & 2635 & 13 & 2586 & $\begin{array}{l}3 \\
3\end{array}$ & $3.29 \%$ \\
\hline 13FR21\#05 & 65 & 108 & $\begin{array}{c}0.6 \\
1\end{array}$ & 0.06240 & $\begin{array}{c}0.002 \\
45\end{array}$ & $\begin{array}{c}0.7845 \\
2\end{array}$ & $\begin{array}{c}0.028 \\
03\end{array}$ & $\begin{array}{c}0.0911 \\
5\end{array}$ & $\begin{array}{c}0.001 \\
94\end{array}$ & 688 & 41 & 588 & 16 & 562 & $\begin{array}{l}1 \\
1\end{array}$ & $4.63 \%$ \\
\hline 13FR21\#06 & 85 & 341 & $\begin{array}{c}0.2 \\
5\end{array}$ & 0.17434 & $\begin{array}{c}0.002 \\
14\end{array}$ & $\begin{array}{c}11.402 \\
12\end{array}$ & $\begin{array}{c}0.132 \\
74\end{array}$ & $\begin{array}{c}0.4741 \\
3\end{array}$ & $\begin{array}{c}0.006 \\
67\end{array}$ & 2600 & 11 & 2557 & 11 & 2502 & 2 & $3.92 \%$ \\
\hline 13FR21\#07 & 96 & 256 & $\begin{array}{c}0.3 \\
8\end{array}$ & 0.18403 & $\begin{array}{c}0.001 \\
95\end{array}$ & $\begin{array}{c}12.401 \\
14\end{array}$ & $\begin{array}{c}0.125 \\
87\end{array}$ & $\begin{array}{c}0.4885 \\
2\end{array}$ & $\begin{array}{c}0.006 \\
44\end{array}$ & 2690 & 10 & 635 & 10 & 2564 & $\begin{array}{l}2 \\
8\end{array}$ & $4.91 \%$ \\
\hline 13FR21\#08 & 114 & 367 & $\begin{array}{c}0.3 \\
1\end{array}$ & 0.06557 & $\begin{array}{c}0.001 \\
27\end{array}$ & $\begin{array}{c}1.0615 \\
7\end{array}$ & $\begin{array}{c}0.018 \\
82\end{array}$ & $\begin{array}{c}0.1173 \\
8\end{array}$ & $\begin{array}{c}0.001 \\
64\end{array}$ & & & & 9 & 715 & 9 & $2.80 \%$ \\
\hline 13FR21\#09 & 53 & 109 & $\begin{array}{c}0.4 \\
9\end{array}$ & 0.07197 & $\begin{array}{c}0.005 \\
42\end{array}$ & $\begin{array}{c}0.8540 \\
7\end{array}$ & $\begin{array}{c}0.061 \\
04\end{array}$ & $\begin{array}{c}0.0860 \\
6\end{array}$ & $\begin{array}{c}0.002 \\
05\end{array}$ & 985 & $\begin{array}{c}15 \\
8\end{array}$ & 627 & 33 & 532 & $\begin{array}{l}1 \\
2\end{array}$ & $17.86 \%$ \\
\hline 13FR21\#10 & 62 & 75 & $\begin{array}{c}0.8 \\
2\end{array}$ & 0.11599 & $\begin{array}{c}0.002 \\
23\end{array}$ & $\begin{array}{c}5.6285 \\
9\end{array}$ & $\begin{array}{c}0.099 \\
95\end{array}$ & $\begin{array}{c}0.3517 \\
9\end{array}$ & $\begin{array}{c}0.005 \\
85\end{array}$ & 1895 & 14 & 1920 & 15 & 1943 & $\begin{array}{l}2 \\
8\end{array}$ & $-2.47 \%$ \\
\hline 13FR21\#11 & 113 & 233 & $\begin{array}{c}0.4 \\
8\end{array}$ & 0.05895 & $\begin{array}{c}0.001 \\
99\end{array}$ & $\begin{array}{l}0.7028 \\
5\end{array}$ & $\begin{array}{c}0.021 \\
72\end{array}$ & $\begin{array}{c}0.0864 \\
3\end{array}$ & $\begin{array}{c}0.001 \\
62\end{array}$ & 565 & 36 & 540 & 13 & 534 & $\begin{array}{l}1 \\
0\end{array}$ & $1.12 \%$ \\
\hline 13FR21\#12 & 115 & 200 & $\begin{array}{c}0.5 \\
7\end{array}$ & 0.17548 & $\begin{array}{c}0.001 \\
96\end{array}$ & $\begin{array}{c}12.191 \\
51\end{array}$ & $\begin{array}{c}0.130 \\
32\end{array}$ & $\begin{array}{c}0.5036 \\
7\end{array}$ & $\begin{array}{c}0.006 \\
78\end{array}$ & 2611 & 10 & 2619 & 10 & 2630 & $\begin{array}{l}2 \\
9\end{array}$ & $-0.72 \%$ \\
\hline 13FR21\#13 & 48 & 198 & $\begin{array}{c}0.2 \\
4\end{array}$ & 0.05712 & $\begin{array}{c}0.001 \\
76\end{array}$ & $\begin{array}{c}0.6342 \\
6\end{array}$ & $\begin{array}{c}0.017 \\
96\end{array}$ & $\begin{array}{c}0.0805 \\
0\end{array}$ & $\begin{array}{c}0.001 \\
40\end{array}$ & 496 & 33 & 499 & 11 & 499 & 8 & $0.00 \%$ \\
\hline 13FR21\#14 & 61 & 120 & $\begin{array}{c}0.5 \\
1\end{array}$ & 0.05869 & $\begin{array}{c}0.002 \\
50\end{array}$ & $\begin{array}{c}0.6292 \\
9\end{array}$ & $\begin{array}{c}0.024 \\
44\end{array}$ & $\begin{array}{c}0.0777 \\
4\end{array}$ & $\begin{array}{c}0.001 \\
72\end{array}$ & 556 & 47 & 496 & 15 & 483 & 1 & $2.69 \%$ \\
\hline 13FR21\#15 & 61 & 210 & $\begin{array}{c}0.2 \\
9\end{array}$ & 0.07139 & $\begin{array}{c}0.002 \\
44\end{array}$ & $\begin{array}{c}1.4844 \\
4\end{array}$ & $\begin{array}{c}0.045 \\
25\end{array}$ & $\begin{array}{c}0.1508 \\
1\end{array}$ & $\begin{array}{c}0.002 \\
32\end{array}$ & 969 & 71 & 924 & 18 & 906 & $\begin{array}{l}1 \\
3\end{array}$ & $1.99 \%$ \\
\hline 13FR21\#16 & 82 & 91 & $\begin{array}{c}0.9 \\
0\end{array}$ & 0.06546 & $\begin{array}{c}0.003 \\
24\end{array}$ & $\begin{array}{c}0.7895 \\
2\end{array}$ & $\begin{array}{c}0.035 \\
62\end{array}$ & $\begin{array}{c}0.0874 \\
4\end{array}$ & $\begin{array}{c}0.002 \\
24\end{array}$ & 789 & 53 & 591 & 20 & 540 & $\begin{array}{l}1 \\
3\end{array}$ & $9.44 \%$ \\
\hline 13FR21\#17 & 20 & 55 & $\begin{array}{c}0.3 \\
7\end{array}$ & 0.05817 & $\begin{array}{c}0.003 \\
28\end{array}$ & $\begin{array}{c}0.7317 \\
3\end{array}$ & $\begin{array}{c}0.037 \\
93\end{array}$ & $\begin{array}{c}0.0912 \\
0\end{array}$ & $\begin{array}{c}0.002 \\
49\end{array}$ & 536 & 67 & 558 & 22 & 563 & $\begin{array}{l}1 \\
5\end{array}$ & $-0.89 \%$ \\
\hline 13FR21\#18 & 78 & 168 & $\begin{array}{c}0.4 \\
7\end{array}$ & 0.05699 & $\begin{array}{c}0.002 \\
31\end{array}$ & $\begin{array}{c}0.6419 \\
3\end{array}$ & $\begin{array}{c}0.023 \\
80\end{array}$ & $\begin{array}{c}0.0816 \\
6\end{array}$ & $\begin{array}{c}0.001 \\
73\end{array}$ & 491 & 46 & 504 & 15 & 506 & $\begin{array}{l}1 \\
0\end{array}$ & $-0.40 \%$ \\
\hline 13FR21\#19 & 66 & 84 & $\begin{array}{c}0.7 \\
8\end{array}$ & 0.05999 & $\begin{array}{c}0.003 \\
69\end{array}$ & $\begin{array}{c}0.7980 \\
9\end{array}$ & $\begin{array}{c}0.044 \\
85\end{array}$ & $\begin{array}{c}0.0964 \\
5\end{array}$ & $\begin{array}{c}0.002 \\
96\end{array}$ & 603 & 70 & 596 & 25 & 594 & $\begin{array}{l}1 \\
7\end{array}$ & $0.34 \%$ \\
\hline 13FR21\#20 & 90 & 330 & $\begin{array}{c}0.2 \\
7\end{array}$ & 0.05903 & $\begin{array}{c}0.001 \\
37\end{array}$ & $\begin{array}{c}0.7918 \\
8\end{array}$ & $\begin{array}{c}0.016 \\
88\end{array}$ & $\begin{array}{c}0.0972 \\
5\end{array}$ & $\begin{array}{c}0.001 \\
46\end{array}$ & 568 & 23 & 592 & 10 & 598 & 9 & $-1.00 \%$ \\
\hline 13FR21\#21 & 95 & 83 & 1.1 & 0.12602 & 0.002 & 6.3298 & 0.114 & 0.3641 & 0.006 & 2043 & 14 & 2023 & 16 & 2002 & 2 & $2.05 \%$ \\
\hline
\end{tabular}




\begin{tabular}{|c|c|c|c|c|c|c|c|c|c|c|c|c|c|c|c|c|}
\hline & & & 5 & & 46 & 0 & 10 & 8 & 24 & & & & & & 9 & \\
\hline 13FR21\#22 & 44 & 62 & $\begin{array}{c}0.7 \\
0\end{array}$ & 0.12935 & $\begin{array}{c}0.002 \\
79\end{array}$ & $\begin{array}{c}6.7094 \\
2\end{array}$ & $\begin{array}{c}0.133 \\
62\end{array}$ & $\begin{array}{c}0.3760 \\
6\end{array}$ & $\begin{array}{c}0.006 \\
96\end{array}$ & 2089 & 16 & 2074 & 18 & 2058 & $\begin{array}{l}3 \\
3\end{array}$ & $1.51 \%$ \\
\hline 13FR21\#23 & 34 & 98 & $\begin{array}{c}0.3 \\
4\end{array}$ & 0.16495 & $\begin{array}{c}0.002 \\
81\end{array}$ & $\begin{array}{c}10.305 \\
03\end{array}$ & $\begin{array}{c}0.164 \\
45\end{array}$ & $\begin{array}{c}0.4529 \\
4\end{array}$ & $\begin{array}{c}0.007 \\
66\end{array}$ & 2507 & 13 & 2463 & 15 & 2408 & $\begin{array}{l}3 \\
4\end{array}$ & $4.11 \%$ \\
\hline 13FR21\#24 & 166 & 172 & $\begin{array}{c}0.9 \\
7\end{array}$ & 0.14251 & $\begin{array}{c}0.001 \\
88\end{array}$ & $\begin{array}{c}7.9592 \\
9\end{array}$ & $\begin{array}{c}0.098 \\
41\end{array}$ & $\begin{array}{c}0.4049 \\
4\end{array}$ & $\begin{array}{c}0.005 \\
67\end{array}$ & 2258 & 11 & 226 & 11 & 2192 & $\begin{array}{l}2 \\
6\end{array}$ & $3.01 \%$ \\
\hline 13FR21\#25 & 20 & 285 & $\begin{array}{c}0.0 \\
7\end{array}$ & 0.05843 & $\begin{array}{c}0.001 \\
66\end{array}$ & $\begin{array}{c}0.6951 \\
9\end{array}$ & $\begin{array}{c}0.018 \\
07\end{array}$ & $\begin{array}{c}0.0862 \\
6\end{array}$ & $\begin{array}{c}0.001 \\
43\end{array}$ & 546 & & & 11 & 533 & 8 & $0.56 \%$ \\
\hline 13FR21\#26 & 35 & 110 & $\begin{array}{c}0.3 \\
2\end{array}$ & 0.05541 & $\begin{array}{c}0.002 \\
16\end{array}$ & $\begin{array}{c}0.6722 \\
6\end{array}$ & $\begin{array}{c}0.023 \\
98\end{array}$ & $\begin{array}{c}0.0879 \\
7\end{array}$ & $\begin{array}{c}0.001 \\
81\end{array}$ & 29 & 44 & 522 & 15 & 544 & $\begin{array}{l}1 \\
1\end{array}$ & $-4.04 \%$ \\
\hline 13FR21\#27 & 208 & 481 & $\begin{array}{c}0.4 \\
3\end{array}$ & 0.05803 & $\begin{array}{c}0.001 \\
33\end{array}$ & $\begin{array}{c}0.7224 \\
0\end{array}$ & $\begin{array}{c}0.015 \\
22\end{array}$ & $\begin{array}{c}0.0902 \\
6\end{array}$ & $\begin{array}{c}0.001 \\
33\end{array}$ & 531 & 23 & 552 & 9 & 557 & 8 & $-0.90 \%$ \\
\hline 13FR21\#28 & 77 & 77 & $\begin{array}{c}0.9 \\
9\end{array}$ & 0.06547 & $\begin{array}{c}0.003 \\
57\end{array}$ & $\begin{array}{c}0.7966 \\
5\end{array}$ & $\begin{array}{c}0.039 \\
30\end{array}$ & $\begin{array}{c}0.0882 \\
2\end{array}$ & $\begin{array}{c}0.002 \\
49\end{array}$ & 789 & 58 & 595 & 22 & 545 & $\begin{array}{l}1 \\
5\end{array}$ & $9.17 \%$ \\
\hline $13 F R 21 \# 29$ & 326 & 471 & $\begin{array}{c}0.6 \\
9\end{array}$ & 0.07518 & $\begin{array}{c}0.001 \\
48\end{array}$ & $\begin{array}{c}0.8692 \\
6\end{array}$ & $\begin{array}{c}0.015 \\
42\end{array}$ & $\begin{array}{c}0.0838 \\
3\end{array}$ & $\begin{array}{c}0.001 \\
20\end{array}$ & 1073 & 16 & 635 & 8 & 519 & 7 & $22.35 \%$ \\
\hline 13FR21\#30 & 52 & 147 & $\begin{array}{c}0.3 \\
6\end{array}$ & 0.05708 & $\begin{array}{c}0.002 \\
39\end{array}$ & $\begin{array}{c}0.6232 \\
2\end{array}$ & $\begin{array}{c}0.023 \\
88\end{array}$ & $\begin{array}{c}0.0791 \\
6\end{array}$ & $\begin{array}{c}0.001 \\
71\end{array}$ & 495 & 48 & 492 & 15 & 491 & $\begin{array}{l}1 \\
0\end{array}$ & $0.20 \%$ \\
\hline 13FR21\#31 & 146 & 230 & $\begin{array}{c}0.6 \\
3\end{array}$ & 0.05730 & $\begin{array}{c}0.002 \\
18\end{array}$ & $\begin{array}{c}0.6138 \\
1\end{array}$ & $\begin{array}{c}0.021 \\
43\end{array}$ & $\begin{array}{c}0.0776 \\
7\end{array}$ & $\begin{array}{c}0.001 \\
55\end{array}$ & 503 & 43 & 486 & 13 & 482 & 9 & $0.83 \%$ \\
\hline 13FR21\#32 & 64 & 115 & $\begin{array}{c}0.5 \\
5\end{array}$ & 0.17137 & $\begin{array}{c}0.002 \\
44\end{array}$ & $\begin{array}{c}11.380 \\
78\end{array}$ & $\begin{array}{c}0.153 \\
59\end{array}$ & $\begin{array}{c}0.4815 \\
2\end{array}$ & $\begin{array}{c}0.007 \\
35\end{array}$ & 2571 & 11 & 2555 & 13 & 2534 & $\begin{array}{l}3 \\
2\end{array}$ & $1.46 \%$ \\
\hline 13FR21\#33 & 100 & 305 & $\begin{array}{c}0.3 \\
3\end{array}$ & 0.06042 & $\begin{array}{c}0.001 \\
56\end{array}$ & $\begin{array}{c}0.8045 \\
7\end{array}$ & $\begin{array}{c}0.018 \\
97\end{array}$ & $\begin{array}{c}0.0965 \\
5\end{array}$ & $\begin{array}{c}0.001 \\
53\end{array}$ & 619 & 26 & 599 & 11 & 594 & 9 & $0.84 \%$ \\
\hline 13FR21\#34 & 58 & 91 & $\begin{array}{c}0.6 \\
4\end{array}$ & 0.05737 & $\begin{array}{c}0.002 \\
77\end{array}$ & $\begin{array}{c}0.6906 \\
9\end{array}$ & $\begin{array}{c}0.030 \\
72\end{array}$ & $\begin{array}{c}0.0873 \\
0\end{array}$ & $\begin{array}{c}0.002 \\
09\end{array}$ & 506 & 57 & 533 & 18 & 540 & $\begin{array}{l}1 \\
2\end{array}$ & $-1.30 \%$ \\
\hline 13FR21\#35 & 83 & 130 & $\begin{array}{c}0.6 \\
4\end{array}$ & 0.06077 & $\begin{array}{c}0.002 \\
37\end{array}$ & $\begin{array}{c}0.7646 \\
1\end{array}$ & $\begin{array}{c}0.027 \\
16\end{array}$ & $\begin{array}{c}0.0912 \\
3\end{array}$ & $\begin{array}{c}0.001 \\
91\end{array}$ & 631 & 42 & 577 & 16 & 563 & $\begin{array}{l}1 \\
1\end{array}$ & $2.49 \%$ \\
\hline 13FR21\#36 & 173 & 168 & $\begin{array}{c}1.0 \\
3\end{array}$ & 0.06280 & $\begin{array}{c}0.002 \\
34\end{array}$ & $\begin{array}{c}0.7199 \\
8\end{array}$ & $\begin{array}{c}0.024 \\
37\end{array}$ & $\begin{array}{c}0.0831 \\
3\end{array}$ & $\begin{array}{c}0.001 \\
70\end{array}$ & 701 & 39 & 551 & 14 & 515 & $\begin{array}{l}1 \\
0\end{array}$ & $6.99 \%$ \\
\hline 13FR21\#37 & 102 & 415 & $\begin{array}{c}0.2 \\
5\end{array}$ & 0.06220 & $\begin{array}{c}0.001 \\
31\end{array}$ & $\begin{array}{c}0.9466 \\
4\end{array}$ & $\begin{array}{c}0.018 \\
30\end{array}$ & $\begin{array}{c}0.1103 \\
5\end{array}$ & $\begin{array}{c}0.001 \\
59\end{array}$ & 681 & 20 & 676 & 10 & 675 & 9 & $0.15 \%$ \\
\hline 13FR21\#38 & 50 & 192 & $\begin{array}{c}0.2 \\
6\end{array}$ & 0.05657 & $\begin{array}{c}0.002 \\
06\end{array}$ & $\begin{array}{c}0.6052 \\
3\end{array}$ & $\begin{array}{c}0.020 \\
23\end{array}$ & $\begin{array}{c}0.0775 \\
8\end{array}$ & $\begin{array}{c}0.001 \\
49\end{array}$ & 475 & 41 & 481 & 13 & 482 & 9 & $-0.21 \%$ \\
\hline
\end{tabular}




\begin{tabular}{|c|c|c|c|c|c|c|c|c|c|c|c|c|c|c|c|c|}
\hline 13FR21\#39 & 38 & 137 & $\begin{array}{c}0.2 \\
7\end{array}$ & 0.05733 & $\begin{array}{c}0.002 \\
50\end{array}$ & $\begin{array}{c}0.6425 \\
5\end{array}$ & $\begin{array}{c}0.025 \\
64\end{array}$ & $\begin{array}{c}0.0812 \\
7\end{array}$ & $\begin{array}{c}0.001 \\
82\end{array}$ & 504 & 50 & 504 & 16 & 504 & $\begin{array}{l}1 \\
1\end{array}$ & $0.00 \%$ \\
\hline 13FR21\#40 & 21 & 183 & $\begin{array}{c}0.1 \\
1\end{array}$ & 0.05805 & $\begin{array}{c}0.001 \\
87\end{array}$ & $\begin{array}{c}0.6748 \\
4\end{array}$ & $\begin{array}{c}0.019 \\
86\end{array}$ & $\begin{array}{c}0.0843 \\
0\end{array}$ & $\begin{array}{c}0.001 \\
51\end{array}$ & 532 & 35 & 524 & 12 & 522 & 9 & $0.38 \%$ \\
\hline 13FR21\#41 & 735 & 281 & $\begin{array}{c}2.6 \\
1\end{array}$ & 0.17835 & $\begin{array}{c}0.001 \\
84\end{array}$ & $\begin{array}{c}11.905 \\
44\end{array}$ & $\begin{array}{c}0.117 \\
29\end{array}$ & $\begin{array}{c}0.4840 \\
5\end{array}$ & $\begin{array}{c}0.006 \\
23\end{array}$ & 2638 & 10 & 2597 & 9 & 2545 & $\begin{array}{l}2 \\
7\end{array}$ & $3.65 \%$ \\
\hline 13FR21\#42 & 319 & 386 & $\begin{array}{c}0.8 \\
3\end{array}$ & 0.05772 & $\begin{array}{c}0.001 \\
51\end{array}$ & $\begin{array}{c}0.6133 \\
4\end{array}$ & $\begin{array}{c}0.014 \\
74\end{array}$ & $\begin{array}{c}0.0770 \\
5\end{array}$ & $\begin{array}{c}0.001 \\
21\end{array}$ & 519 & & & 9 & 478 & 7 & $1.67 \%$ \\
\hline 13FR21\#43 & 33 & 113 & $\begin{array}{c}0.3 \\
0\end{array}$ & 0.05625 & $\begin{array}{c}0.004 \\
06\end{array}$ & $\begin{array}{c}0.6021 \\
9\end{array}$ & $\begin{array}{c}0.041 \\
16\end{array}$ & $\begin{array}{c}0.0776 \\
5\end{array}$ & $\begin{array}{c}0.001 \\
82\end{array}$ & & & & 26 & 482 & 1 & $-0.62 \%$ \\
\hline 13FR21\#44 & 290 & 348 & $\begin{array}{c}0.8 \\
3\end{array}$ & 0.06023 & $\begin{array}{c}0.001 \\
50\end{array}$ & $\begin{array}{c}0.7286 \\
3\end{array}$ & $\begin{array}{c}0.016 \\
61\end{array}$ & $\begin{array}{c}0.0877 \\
2\end{array}$ & $\begin{array}{c}0.001 \\
36\end{array}$ & 612 & 25 & 556 & 10 & 542 & 8 & $2.58 \%$ \\
\hline 13FR21\#45 & 48 & 144 & $\begin{array}{c}0.3 \\
3\end{array}$ & 0.05698 & $\begin{array}{c}0.002 \\
59\end{array}$ & $\begin{array}{c}0.6159 \\
9\end{array}$ & $\begin{array}{c}0.025 \\
58\end{array}$ & $\begin{array}{c}0.0783 \\
9\end{array}$ & $\begin{array}{c}0.001 \\
80\end{array}$ & 491 & 52 & 487 & 16 & 487 & 1 & $0.00 \%$ \\
\hline 13FR21\#46 & 294 & 599 & $\begin{array}{c}0.4 \\
9\end{array}$ & 0.05988 & $\begin{array}{c}0.001 \\
24\end{array}$ & $\begin{array}{c}0.7372 \\
5\end{array}$ & $\begin{array}{c}0.013 \\
96\end{array}$ & $\begin{array}{c}0.0892 \\
8\end{array}$ & $\begin{array}{c}0.001 \\
25\end{array}$ & 599 & 19 & 561 & 8 & 551 & 7 & $1.81 \%$ \\
\hline 13FR21\#47 & 40 & 118 & $\begin{array}{c}0.3 \\
4\end{array}$ & 0.06328 & $\begin{array}{c}0.003 \\
38\end{array}$ & $\begin{array}{c}0.6914 \\
7\end{array}$ & $\begin{array}{c}0.033 \\
54\end{array}$ & $\begin{array}{c}0.0792 \\
4\end{array}$ & $\begin{array}{c}0.002 \\
14\end{array}$ & 718 & 58 & 534 & 20 & 492 & 3 & $8.54 \%$ \\
\hline 13FR21\#48 & 29 & 60 & $\begin{array}{c}0.4 \\
9\end{array}$ & 0.05792 & $\begin{array}{c}0.003 \\
11\end{array}$ & $\begin{array}{c}0.7021 \\
9\end{array}$ & $\begin{array}{c}0.034 \\
53\end{array}$ & $\begin{array}{c}0.0879 \\
1\end{array}$ & $\begin{array}{c}0.002 \\
34\end{array}$ & 527 & 62 & 540 & 21 & 543 & $\begin{array}{l}1 \\
4\end{array}$ & $-0.55 \%$ \\
\hline 13FR21\#49 & 30 & 92 & $\begin{array}{c}0.3 \\
2\end{array}$ & 0.05707 & $\begin{array}{c}0.002 \\
81\end{array}$ & $\begin{array}{c}0.6118 \\
4\end{array}$ & $\begin{array}{c}0.027 \\
63\end{array}$ & $\begin{array}{c}0.0777 \\
5\end{array}$ & $\begin{array}{c}0.001 \\
90\end{array}$ & 494 & 58 & 485 & 17 & 483 & 1 & $0.41 \%$ \\
\hline 13FR21\#50 & 276 & 280 & $\begin{array}{c}0.9 \\
9\end{array}$ & 0.05972 & $\begin{array}{c}0.001 \\
49\end{array}$ & $\begin{array}{c}0.7782 \\
6\end{array}$ & $\begin{array}{c}0.017 \\
74\end{array}$ & $\begin{array}{c}0.0945 \\
0\end{array}$ & $\begin{array}{c}0.001 \\
46\end{array}$ & 593 & 25 & 584 & 10 & 582 & 9 & $0.34 \%$ \\
\hline 13FR21\#51 & 125 & 199 & $\begin{array}{c}0.6 \\
3\end{array}$ & 0.06114 & $\begin{array}{c}0.001 \\
64\end{array}$ & $\begin{array}{c}0.8541 \\
0\end{array}$ & $\begin{array}{c}0.021 \\
03\end{array}$ & $\begin{array}{c}0.1013 \\
0\end{array}$ & $\begin{array}{c}0.001 \\
66\end{array}$ & 644 & 27 & 627 & 12 & 622 & $\begin{array}{l}1 \\
0\end{array}$ & $0.80 \%$ \\
\hline 13FR21\#52 & 329 & 372 & $\begin{array}{c}0.8 \\
8\end{array}$ & 0.05438 & $\begin{array}{c}0.001 \\
67\end{array}$ & $\begin{array}{c}0.4793 \\
7\end{array}$ & $\begin{array}{c}0.013 \\
52\end{array}$ & $\begin{array}{c}0.0639 \\
2\end{array}$ & $\begin{array}{c}0.001 \\
08\end{array}$ & 387 & 34 & 398 & 9 & 399 & 7 & $-0.25 \%$ \\
\hline 13FR21\#53 & 62 & 187 & $\begin{array}{c}0.3 \\
3\end{array}$ & 0.05822 & $\begin{array}{c}0.001 \\
91\end{array}$ & $\begin{array}{c}0.6803 \\
0\end{array}$ & $\begin{array}{c}0.020 \\
48\end{array}$ & $\begin{array}{c}0.0847 \\
5\end{array}$ & $\begin{array}{c}0.001 \\
54\end{array}$ & 538 & 36 & 527 & 12 & 524 & 9 & $0.57 \%$ \\
\hline 13FR21\#54 & 53 & 164 & $\begin{array}{c}0.3 \\
3\end{array}$ & 0.05828 & $\begin{array}{c}0.002 \\
06\end{array}$ & $\begin{array}{c}0.6418 \\
2\end{array}$ & $\begin{array}{c}0.020 \\
76\end{array}$ & $\begin{array}{c}0.0798 \\
7\end{array}$ & $\begin{array}{c}0.001 \\
51\end{array}$ & 540 & 39 & 503 & 13 & 495 & 9 & $1.62 \%$ \\
\hline 13FR21\#55 & 94 & 338 & $\begin{array}{c}0.2 \\
8\end{array}$ & 0.05719 & $\begin{array}{c}0.001 \\
55\end{array}$ & $\begin{array}{c}0.6343 \\
9\end{array}$ & $\begin{array}{c}0.015 \\
76\end{array}$ & $\begin{array}{c}0.0804 \\
5\end{array}$ & $\begin{array}{c}0.001 \\
29\end{array}$ & 499 & 28 & 499 & 10 & 499 & 8 & $0.00 \%$ \\
\hline 13FR21\#56 & 45 & 128 & 0.3 & 0.05740 & 0.002 & 0.6182 & 0.025 & 0.0781 & 0.001 & 507 & 53 & 489 & 16 & 485 & 1 & $0.82 \%$ \\
\hline
\end{tabular}




\begin{tabular}{|c|c|c|c|c|c|c|c|c|c|c|c|c|c|c|c|c|}
\hline & & & 5 & & 62 & 3 & 83 & 1 & 79 & & & & & & 1 & \\
\hline 13FR21\#57 & 524 & 297 & $\begin{array}{c}1.7 \\
7\end{array}$ & 0.06177 & $\begin{array}{c}0.001 \\
79\end{array}$ & $\begin{array}{c}0.8578 \\
2\end{array}$ & $\begin{array}{c}0.022 \\
75\end{array}$ & $\begin{array}{c}0.1007 \\
2\end{array}$ & $\begin{array}{c}0.001 \\
73\end{array}$ & 666 & 29 & 629 & 12 & 619 & $\begin{array}{l}1 \\
0\end{array}$ & $1.62 \%$ \\
\hline 13FR21\#58 & 90 & 218 & $\begin{array}{c}0.4 \\
1\end{array}$ & 0.09894 & $\begin{array}{c}0.001 \\
83\end{array}$ & $\begin{array}{c}2.1080 \\
8\end{array}$ & $\begin{array}{c}0.034 \\
73\end{array}$ & $\begin{array}{c}0.1545 \\
2\end{array}$ & $\begin{array}{c}0.002 \\
28\end{array}$ & 1604 & 14 & 1151 & 11 & 926 & $\begin{array}{l}1 \\
3\end{array}$ & $24.30 \%$ \\
\hline 13FR21\#59 & 106 & 109 & $\begin{array}{c}0.9 \\
7\end{array}$ & 0.05907 & $\begin{array}{c}0.002 \\
98\end{array}$ & $\begin{array}{c}0.7418 \\
8\end{array}$ & $\begin{array}{c}0.034 \\
20\end{array}$ & $\begin{array}{c}0.0910 \\
9\end{array}$ & $\begin{array}{c}0.002 \\
33\end{array}$ & 570 & 57 & & 20 & 562 & $\begin{array}{l}1 \\
4\end{array}$ & $0.36 \%$ \\
\hline 13FR21\#60 & 56 & 89 & $\begin{array}{c}0.6 \\
3\end{array}$ & 0.10810 & $\begin{array}{c}0.002 \\
41\end{array}$ & $\begin{array}{c}4.6946 \\
1\end{array}$ & $\begin{array}{c}0.096 \\
01\end{array}$ & $\begin{array}{c}0.3149 \\
6\end{array}$ & $\begin{array}{c}0.005 \\
60\end{array}$ & 1768 & & & 17 & 1765 & $\begin{array}{l}2 \\
7\end{array}$ & $0.17 \%$ \\
\hline 13FR21\#61 & 26 & 32 & $\begin{array}{c}0.8 \\
1\end{array}$ & 0.08642 & $\begin{array}{c}0.004 \\
08\end{array}$ & $\begin{array}{c}2.7092 \\
5\end{array}$ & $\begin{array}{c}0.116 \\
57\end{array}$ & $\begin{array}{c}0.2273 \\
6\end{array}$ & $\begin{array}{c}0.006 \\
68\end{array}$ & 1348 & 41 & 1331 & 32 & 1321 & $\begin{array}{l}3 \\
5\end{array}$ & $2.04 \%$ \\
\hline 13FR21\#62 & 158 & 191 & $\begin{array}{c}0.8 \\
2\end{array}$ & 0.05778 & $\begin{array}{c}0.001 \\
79\end{array}$ & $\begin{array}{c}0.7403 \\
2\end{array}$ & $\begin{array}{c}0.021 \\
07\end{array}$ & $\begin{array}{c}0.0929 \\
2\end{array}$ & 0.001 & 521 & 33 & 563 & 12 & 573 & $\begin{array}{l}1 \\
0\end{array}$ & $-1.75 \%$ \\
\hline 13FR21\#63 & 52 & 242 & $\begin{array}{c}0.2 \\
1\end{array}$ & 0.05847 & $\begin{array}{c}0.001 \\
87\end{array}$ & $\begin{array}{c}0.6504 \\
3\end{array}$ & $\begin{array}{c}0.019 \\
02\end{array}$ & $\begin{array}{c}0.0806 \\
9\end{array}$ & $\begin{array}{c}0.001 \\
44\end{array}$ & 547 & 34 & 509 & 12 & 500 & 9 & $1.80 \%$ \\
\hline 13FR21\#64 & 52 & 164 & $\begin{array}{c}0.3 \\
2\end{array}$ & 0.05926 & $\begin{array}{c}0.002 \\
73\end{array}$ & $\begin{array}{c}0.7587 \\
9\end{array}$ & $\begin{array}{c}0.032 \\
07\end{array}$ & $\begin{array}{c}0.0928 \\
7\end{array}$ & $\begin{array}{c}0.002 \\
19\end{array}$ & 577 & 52 & 573 & 19 & 572 & $\begin{array}{l}1 \\
3\end{array}$ & $0.17 \%$ \\
\hline 13FR21\#65 & 424 & 285 & $\begin{array}{c}1.4 \\
9\end{array}$ & 0.05841 & $\begin{array}{c}0.001 \\
81\end{array}$ & $\begin{array}{c}0.7095 \\
2\end{array}$ & $\begin{array}{c}0.020 \\
18\end{array}$ & $\begin{array}{c}0.0881 \\
1\end{array}$ & $\begin{array}{c}0.001 \\
55\end{array}$ & 545 & 33 & 544 & 12 & 544 & 9 & $0.00 \%$ \\
\hline 13FR21\#66 & 52 & 153 & $\begin{array}{c}0.3 \\
4\end{array}$ & 0.06145 & $\begin{array}{c}0.001 \\
81\end{array}$ & $\begin{array}{c}0.8876 \\
4\end{array}$ & $\begin{array}{c}0.023 \\
94\end{array}$ & $\begin{array}{c}0.1047 \\
7\end{array}$ & $\begin{array}{c}0.001 \\
81\end{array}$ & 655 & 30 & 645 & 13 & 642 & $\begin{array}{l}1 \\
1\end{array}$ & $0.47 \%$ \\
\hline 13FR21\#67 & 30 & 40 & $\begin{array}{c}0.7 \\
5\end{array}$ & 0.06548 & $\begin{array}{c}0.004 \\
25\end{array}$ & $\begin{array}{c}0.8195 \\
0\end{array}$ & $\begin{array}{c}0.048 \\
31\end{array}$ & $\begin{array}{c}0.0907 \\
8\end{array}$ & $\begin{array}{c}0.002 \\
95\end{array}$ & 790 & 71 & 608 & 27 & 560 & $\begin{array}{l}1 \\
7\end{array}$ & $8.57 \%$ \\
\hline 13FR21\#68 & 175 & 293 & $\begin{array}{c}0.6 \\
0\end{array}$ & 0.06247 & $\begin{array}{c}0.001 \\
58\end{array}$ & $\begin{array}{c}0.8332 \\
4\end{array}$ & $\begin{array}{c}0.019 \\
27\end{array}$ & $\begin{array}{c}0.0967 \\
4\end{array}$ & $\begin{array}{c}0.001 \\
52\end{array}$ & 690 & 25 & 615 & 11 & 595 & 9 & $3.36 \%$ \\
\hline 13FR21\#69 & 56 & 179 & $\begin{array}{c}0.3 \\
2\end{array}$ & 0.05874 & $\begin{array}{c}0.002 \\
02\end{array}$ & $\begin{array}{c}0.7178 \\
7\end{array}$ & $\begin{array}{c}0.022 \\
66\end{array}$ & $\begin{array}{c}0.0886 \\
4\end{array}$ & $\begin{array}{c}0.001 \\
67\end{array}$ & 557 & 37 & 549 & 13 & 547 & $\begin{array}{l}1 \\
0\end{array}$ & $0.37 \%$ \\
\hline 13FR21\#70 & 35 & 165 & $\begin{array}{c}0.2 \\
1\end{array}$ & 0.05778 & $\begin{array}{c}0.003 \\
38\end{array}$ & $\begin{array}{c}0.6194 \\
9\end{array}$ & $\begin{array}{c}0.034 \\
11\end{array}$ & $\begin{array}{c}0.0777 \\
6\end{array}$ & $\begin{array}{c}0.001 \\
55\end{array}$ & 522 & $\begin{array}{c}13 \\
2\end{array}$ & 490 & 21 & 483 & 9 & $1.45 \%$ \\
\hline 13FR21\#71 & 23 & 74 & $\begin{array}{c}0.3 \\
1\end{array}$ & 0.12760 & $\begin{array}{c}0.002 \\
97\end{array}$ & $\begin{array}{c}6.4975 \\
5\end{array}$ & $\begin{array}{c}0.139 \\
69\end{array}$ & $\begin{array}{c}0.3693 \\
5\end{array}$ & $\begin{array}{c}0.007 \\
16\end{array}$ & 2065 & 17 & 2046 & 19 & 2026 & $\begin{array}{l}3 \\
4\end{array}$ & $1.92 \%$ \\
\hline 13FR21\#72 & 72 & 69 & $\begin{array}{c}1.0 \\
4\end{array}$ & 0.05944 & $\begin{array}{c}0.003 \\
12\end{array}$ & $\begin{array}{c}0.7181 \\
0\end{array}$ & $\begin{array}{c}0.034 \\
78\end{array}$ & $\begin{array}{c}0.0876 \\
3\end{array}$ & $\begin{array}{c}0.002 \\
21\end{array}$ & 583 & 62 & 550 & 21 & 542 & $\begin{array}{l}1 \\
3\end{array}$ & $1.48 \%$ \\
\hline 13FR21\#73 & 58 & 217 & $\begin{array}{c}0.2 \\
7\end{array}$ & 0.09834 & $\begin{array}{c}0.002 \\
53\end{array}$ & $\begin{array}{c}1.9966 \\
2\end{array}$ & $\begin{array}{c}0.042 \\
75\end{array}$ & $\begin{array}{c}0.1472 \\
6\end{array}$ & $\begin{array}{c}0.002 \\
11\end{array}$ & 1593 & 49 & 1114 & 14 & 886 & $\begin{array}{l}1 \\
2\end{array}$ & $25.73 \%$ \\
\hline
\end{tabular}




\begin{tabular}{|c|c|c|c|c|c|c|c|c|c|c|c|c|c|c|c|c|}
\hline 13FR21\#74 & 36 & 126 & $\begin{array}{c}0.2 \\
9\end{array}$ & 0.06134 & $\begin{array}{c}0.002 \\
60\end{array}$ & $\begin{array}{c}0.6846 \\
6\end{array}$ & $\begin{array}{c}0.026 \\
38\end{array}$ & $\begin{array}{c}0.0809 \\
6\end{array}$ & $\begin{array}{c}0.001 \\
80\end{array}$ & 651 & 46 & 530 & 16 & 502 & $\begin{array}{l}1 \\
1\end{array}$ & $5.58 \%$ \\
\hline 13FR21\#75 & 66 & 111 & $\begin{array}{c}0.5 \\
9\end{array}$ & 0.06056 & $\begin{array}{c}0.002 \\
53\end{array}$ & $\begin{array}{c}0.8300 \\
7\end{array}$ & $\begin{array}{c}0.031 \\
75\end{array}$ & $\begin{array}{c}0.0994 \\
2\end{array}$ & $\begin{array}{c}0.002 \\
18\end{array}$ & 624 & 46 & 614 & 18 & 611 & $\begin{array}{l}1 \\
3\end{array}$ & $0.49 \%$ \\
\hline 13FR21\#76 & 126 & 133 & $\begin{array}{c}0.9 \\
4\end{array}$ & 0.12436 & $\begin{array}{c}0.001 \\
83\end{array}$ & $\begin{array}{c}6.5303 \\
9\end{array}$ & $\begin{array}{c}0.089 \\
40\end{array}$ & $\begin{array}{c}0.3809 \\
1\end{array}$ & $\begin{array}{c}0.005 \\
45\end{array}$ & 2020 & 11 & 2050 & 12 & 2081 & $\begin{array}{l}2 \\
5\end{array}$ & $-2.93 \%$ \\
\hline 13FR21\#77 & 71 & 146 & $\begin{array}{c}0.4 \\
9\end{array}$ & 0.05433 & $\begin{array}{c}0.002 \\
08\end{array}$ & $\begin{array}{c}0.6004 \\
4\end{array}$ & $\begin{array}{c}0.021 \\
18\end{array}$ & $\begin{array}{c}0.0801 \\
7\end{array}$ & $\begin{array}{c}0.001 \\
57\end{array}$ & 385 & & & 13 & 497 & 9 & $-3.82 \%$ \\
\hline 13FR21\#78 & 80 & 89 & $\begin{array}{c}0.9 \\
0\end{array}$ & 0.05726 & $\begin{array}{c}0.002 \\
52\end{array}$ & $\begin{array}{c}0.6924 \\
6\end{array}$ & $\begin{array}{c}0.027 \\
92\end{array}$ & $\begin{array}{c}0.0877 \\
2\end{array}$ & $\begin{array}{c}0.001 \\
97\end{array}$ & & & 534 & 17 & 542 & 1 & $-1.48 \%$ \\
\hline 13FR21\#79 & 44 & 329 & $\begin{array}{c}0.1 \\
3\end{array}$ & 0.05614 & $\begin{array}{c}0.001 \\
28\end{array}$ & $\begin{array}{c}0.5901 \\
0\end{array}$ & $\begin{array}{c}0.012 \\
35\end{array}$ & $\begin{array}{c}0.0762 \\
5\end{array}$ & $\begin{array}{c}0.001 \\
10\end{array}$ & 458 & 23 & 471 & 8 & 474 & 7 & $-0.63 \%$ \\
\hline 13FR21\#80 & 39 & 79 & $\begin{array}{c}0.5 \\
0\end{array}$ & 0.13279 & $\begin{array}{c}0.002 \\
86\end{array}$ & $\begin{array}{c}6.8521 \\
3\end{array}$ & $\begin{array}{c}0.136 \\
00\end{array}$ & $\begin{array}{c}0.3743 \\
1\end{array}$ & $\begin{array}{c}0.006 \\
92\end{array}$ & 2135 & 16 & 2092 & 18 & 2050 & 3 & $4.15 \%$ \\
\hline 13FR21\#81 & 57 & 80 & $\begin{array}{c}0.7 \\
1\end{array}$ & 0.05986 & $\begin{array}{c}0.002 \\
85\end{array}$ & $\begin{array}{c}0.6934 \\
1\end{array}$ & $\begin{array}{c}0.030 \\
18\end{array}$ & $\begin{array}{c}0.0840 \\
3\end{array}$ & $\begin{array}{c}0.002 \\
02\end{array}$ & 599 & 54 & 535 & 18 & 520 & $\begin{array}{l}1 \\
2\end{array}$ & $2.88 \%$ \\
\hline 13FR21\#82 & 206 & 494 & $\begin{array}{c}0.4 \\
2\end{array}$ & 0.06054 & $\begin{array}{c}0.001 \\
10\end{array}$ & $\begin{array}{c}0.8163 \\
0\end{array}$ & $\begin{array}{c}0.013 \\
67\end{array}$ & $\begin{array}{c}0.0978 \\
1\end{array}$ & $\begin{array}{c}0.001 \\
30\end{array}$ & 623 & 17 & 606 & 8 & 602 & 8 & $0.66 \%$ \\
\hline 13FR21\#83 & 132 & 351 & $\begin{array}{c}0.3 \\
8\end{array}$ & 0.07721 & $\begin{array}{c}0.001 \\
06\end{array}$ & $\begin{array}{c}2.0873 \\
9\end{array}$ & $\begin{array}{c}0.026 \\
60\end{array}$ & $\begin{array}{c}0.1961 \\
3\end{array}$ & $\begin{array}{c}0.002 \\
45\end{array}$ & 1127 & 12 & 1145 & 9 & 1154 & 1 & $-2.34 \%$ \\
\hline 13FR21\#84 & 20 & 67 & $\begin{array}{c}0.3 \\
0\end{array}$ & 0.15464 & $\begin{array}{c}0.002 \\
88\end{array}$ & $\begin{array}{c}9.3793 \\
4\end{array}$ & $\begin{array}{c}0.163 \\
04\end{array}$ & $\begin{array}{c}0.4399 \\
8\end{array}$ & $\begin{array}{c}0.007 \\
72\end{array}$ & 2398 & 14 & 2376 & 16 & 2351 & 3 & $2.00 \%$ \\
\hline 13FR21\#85 & 166 & 352 & $\begin{array}{c}0.4 \\
7\end{array}$ & 0.17881 & $\begin{array}{c}0.002 \\
92\end{array}$ & $\begin{array}{c}11.816 \\
81\end{array}$ & $\begin{array}{c}0.132 \\
15\end{array}$ & $\begin{array}{c}0.4793 \\
1\end{array}$ & $\begin{array}{c}0.005 \\
70\end{array}$ & 2642 & 28 & 2590 & 10 & 2524 & $\begin{array}{l}2 \\
5\end{array}$ & $4.68 \%$ \\
\hline $13 F R 21 \# 86$ & 1531 & 836 & $\begin{array}{c}1.8 \\
3\end{array}$ & 00673 & $\begin{array}{c}0.001 \\
05\end{array}$ & $\begin{array}{c}0.6584 \\
2\end{array}$ & $\begin{array}{c}0.009 \\
33\end{array}$ & $\begin{array}{c}0.0709 \\
4\end{array}$ & $\begin{array}{c}0.000 \\
89\end{array}$ & 848 & 13 & 514 & 6 & 442 & 5 & $16.29 \%$ \\
\hline 13FR21\#87 & 127 & 108 & $\begin{array}{c}1.1 \\
7\end{array}$ & 0.06406 & $\begin{array}{c}0.002 \\
51\end{array}$ & $\begin{array}{c}0.8195 \\
2\end{array}$ & $\begin{array}{c}0.029 \\
35\end{array}$ & $\begin{array}{c}0.0928 \\
1\end{array}$ & $\begin{array}{c}0.001 \\
95\end{array}$ & 744 & 42 & 608 & 16 & 572 & $\begin{array}{l}1 \\
2\end{array}$ & $6.29 \%$ \\
\hline 13FR21\#88 & 64 & 43 & $\begin{array}{c}1.4 \\
8\end{array}$ & 0.06766 & $\begin{array}{c}0.003 \\
37\end{array}$ & $\begin{array}{c}1.3349 \\
9\end{array}$ & $\begin{array}{c}0.060 \\
71\end{array}$ & $\begin{array}{c}0.1431 \\
5\end{array}$ & $\begin{array}{c}0.003 \\
88\end{array}$ & 858 & 51 & 861 & 26 & 862 & $\begin{array}{l}2 \\
2\end{array}$ & $-0.12 \%$ \\
\hline 13FR21\#89 & 266 & 305 & $\begin{array}{c}0.8 \\
7\end{array}$ & 0.06099 & $\begin{array}{c}0.001 \\
30\end{array}$ & $\begin{array}{c}0.7411 \\
3\end{array}$ & $\begin{array}{c}0.014 \\
50\end{array}$ & $\begin{array}{c}0.0881 \\
5\end{array}$ & $\begin{array}{c}0.001 \\
25\end{array}$ & 639 & 20 & 563 & 8 & 545 & 7 & $3.30 \%$ \\
\hline 13FR21\#90 & 92 & 177 & $\begin{array}{c}0.5 \\
2\end{array}$ & 0.05777 & $\begin{array}{c}0.002 \\
40\end{array}$ & $\begin{array}{c}0.6320 \\
5\end{array}$ & $\begin{array}{c}0.024 \\
02\end{array}$ & $\begin{array}{c}0.0793 \\
7\end{array}$ & $\begin{array}{c}0.001 \\
70\end{array}$ & 521 & 47 & 497 & 15 & 492 & $\begin{array}{l}1 \\
0\end{array}$ & $1.02 \%$ \\
\hline 13FR21\#91 & 94 & 203 & 0.4 & 0.05871 & 0.001 & 0.6835 & 0.018 & 0.0844 & 0.001 & 556 & 31 & 529 & 11 & 523 & 9 & $1.15 \%$ \\
\hline
\end{tabular}




\begin{tabular}{|c|c|c|c|c|c|c|c|c|c|c|c|c|c|c|c|c|}
\hline & & & 6 & & 74 & 5 & 53 & 7 & 43 & & & & & & & \\
\hline 13FR21\#92 & 90 & 303 & $\begin{array}{c}0.3 \\
0\end{array}$ & 0.11050 & $\begin{array}{c}0.002 \\
17\end{array}$ & $\begin{array}{c}3.2976 \\
3\end{array}$ & $\begin{array}{c}0.050 \\
17\end{array}$ & $\begin{array}{c}0.2164 \\
5\end{array}$ & $\begin{array}{c}0.002 \\
70\end{array}$ & 1808 & 37 & 1480 & 12 & 1263 & $\begin{array}{l}1 \\
4\end{array}$ & $43.15 \%$ \\
\hline 13FR21\#93 & 76 & 177 & $\begin{array}{c}0.4 \\
3\end{array}$ & 0.05811 & $\begin{array}{c}0.001 \\
89\end{array}$ & $\begin{array}{c}0.6681 \\
3\end{array}$ & $\begin{array}{c}0.019 \\
89\end{array}$ & $\begin{array}{c}0.0834 \\
1\end{array}$ & $\begin{array}{c}0.001 \\
50\end{array}$ & 534 & 35 & 520 & 12 & 516 & 9 & $0.78 \%$ \\
\hline 13FR21\#94 & 52 & 181 & $\begin{array}{c}0.2 \\
9\end{array}$ & 0.05853 & $\begin{array}{c}0.001 \\
78\end{array}$ & $\begin{array}{c}0.7391 \\
7\end{array}$ & $\begin{array}{c}0.020 \\
67\end{array}$ & $\begin{array}{c}0.0916 \\
2\end{array}$ & $\begin{array}{c}0.001 \\
58\end{array}$ & 550 & 32 & & 12 & 565 & 9 & $-0.53 \%$ \\
\hline 13FR21\#95 & 47 & 113 & $\begin{array}{c}0.4 \\
1\end{array}$ & 0.05926 & $\begin{array}{c}0.002 \\
26\end{array}$ & $\begin{array}{c}0.7798 \\
6\end{array}$ & $\begin{array}{c}0.027 \\
17\end{array}$ & $\begin{array}{c}0.0954 \\
9\end{array}$ & $\begin{array}{c}0.001 \\
95\end{array}$ & 5 & & & 16 & 588 & $\begin{array}{l}1 \\
1\end{array}$ & $-0.51 \%$ \\
\hline 13FR21\#96 & 22 & 81 & $\begin{array}{c}0.2 \\
7\end{array}$ & 0.05788 & $\begin{array}{c}0.003 \\
00\end{array}$ & $\begin{array}{c}0.6218 \\
2\end{array}$ & $\begin{array}{c}0.029 \\
78\end{array}$ & $\begin{array}{c}0.0779 \\
4\end{array}$ & $\begin{array}{c}0.001 \\
92\end{array}$ & & 63 & 491 & 19 & 484 & $\begin{array}{l}1 \\
1\end{array}$ & $1.45 \%$ \\
\hline 13FR21\#97 & 37 & 106 & $\begin{array}{c}0.3 \\
5\end{array}$ & 0.05868 & $\begin{array}{c}0.002 \\
52\end{array}$ & $\begin{array}{c}0.6081 \\
6\end{array}$ & $\begin{array}{c}0.023 \\
82\end{array}$ & $\begin{array}{c}0.0752 \\
0\end{array}$ & $\begin{array}{c}0.001 \\
66\end{array}$ & 555 & 48 & 482 & 15 & 467 & $\begin{array}{l}1 \\
0\end{array}$ & $3.21 \%$ \\
\hline 13FR21\#98 & 309 & 322 & $\begin{array}{c}0.9 \\
6\end{array}$ & 0.06108 & $\begin{array}{c}0.001 \\
25\end{array}$ & $\begin{array}{c}0.7307 \\
5\end{array}$ & $\begin{array}{c}0.013 \\
70\end{array}$ & $\begin{array}{c}0.0868 \\
0\end{array}$ & $\begin{array}{c}0.001 \\
20\end{array}$ & 642 & 19 & 557 & 8 & 537 & 7 & $3.72 \%$ \\
\hline 13FR21\#99 & 288 & 188 & $\begin{array}{c}1.5 \\
3\end{array}$ & 0.06033 & $\begin{array}{c}0.001 \\
72\end{array}$ & $\begin{array}{c}0.8241 \\
5\end{array}$ & $\begin{array}{c}0.021 \\
53\end{array}$ & $\begin{array}{c}0.0991 \\
2\end{array}$ & $\begin{array}{c}0.001 \\
66\end{array}$ & 615 & 29 & 610 & 12 & 609 & $\begin{array}{l}1 \\
0\end{array}$ & $0.16 \%$ \\
\hline $\begin{array}{l}\text { 13FR21\#10 } \\
0\end{array}$ & 90 & 338 & $\begin{array}{c}0.2 \\
7\end{array}$ & 0.06574 & $\begin{array}{c}0.001 \\
98\end{array}$ & 1.2062 & $\begin{array}{c}0.032 \\
44\end{array}$ & $\begin{array}{c}0.1330 \\
8\end{array}$ & $\begin{array}{c}0.001 \\
82\end{array}$ & 798 & 65 & 803 & 15 & 805 & $\begin{array}{l}1 \\
0\end{array}$ & $-0.25 \%$ \\
\hline \multicolumn{17}{|l|}{ OUT } \\
\hline OUT\#01 & 146 & 168 & $\begin{array}{c}0.8 \\
7\end{array}$ & 0.06182 & $\begin{array}{c}0.003 \\
64\end{array}$ & $\begin{array}{c}0.7835 \\
1\end{array}$ & $\begin{array}{c}0.044 \\
00\end{array}$ & $\begin{array}{c}0.0919 \\
1\end{array}$ & $\begin{array}{c}0.002 \\
05\end{array}$ & 668 & 82 & 587 & 25 & 567 & $\begin{array}{l}1 \\
2\end{array}$ & $3.53 \%$ \\
\hline OUT\#02 & 83 & 66 & $\begin{array}{c}1.2 \\
6\end{array}$ & 0.06237 & $\begin{array}{c}0.005 \\
84\end{array}$ & $\begin{array}{c}0.9063 \\
5\end{array}$ & $\begin{array}{c}0.081 \\
23\end{array}$ & $\begin{array}{c}0.1053 \\
8\end{array}$ & $\begin{array}{c}0.003 \\
56\end{array}$ & 687 & $\begin{array}{c}13 \\
4\end{array}$ & 655 & 43 & 646 & $\begin{array}{l}2 \\
1\end{array}$ & $1.39 \%$ \\
\hline OUT\#03 & 50 & 77 & $\begin{array}{c}0.6 \\
5\end{array}$ & 0.11789 & $\begin{array}{c}0.004 \\
17\end{array}$ & $\begin{array}{c}5.6075 \\
0\end{array}$ & $\begin{array}{c}0.190 \\
53\end{array}$ & $\begin{array}{c}0.3449 \\
2\end{array}$ & $\begin{array}{c}0.007 \\
25\end{array}$ & 1924 & 32 & 1917 & 29 & 1910 & $\begin{array}{l}3 \\
5\end{array}$ & $0.73 \%$ \\
\hline OUT\#04 & 442 & 875 & $\begin{array}{c}0.5 \\
1\end{array}$ & 0.07002 & $\begin{array}{c}0.007 \\
21\end{array}$ & $\begin{array}{c}0.7487 \\
0\end{array}$ & $\begin{array}{c}0.074 \\
35\end{array}$ & $\begin{array}{c}0.0775 \\
5\end{array}$ & $\begin{array}{c}0.002 \\
11\end{array}$ & 929 & $\begin{array}{c}22 \\
0\end{array}$ & 567 & 43 & 482 & $\begin{array}{l}1 \\
3\end{array}$ & $17.63 \%$ \\
\hline OUT\#05 & 182 & 309 & $\begin{array}{c}0.5 \\
9\end{array}$ & 0.06050 & $\begin{array}{c}0.002 \\
81\end{array}$ & $\begin{array}{c}0.7639 \\
2\end{array}$ & $\begin{array}{c}0.033 \\
81\end{array}$ & $\begin{array}{c}0.0915 \\
7\end{array}$ & $\begin{array}{c}0.001 \\
67\end{array}$ & 622 & 64 & 576 & 19 & 565 & $\begin{array}{l}1 \\
0\end{array}$ & $1.95 \%$ \\
\hline OUT\#06 & 34 & 37 & $\begin{array}{c}0.9 \\
0\end{array}$ & 0.18798 & $\begin{array}{c}0.006 \\
84\end{array}$ & $\begin{array}{c}12.667 \\
53\end{array}$ & $\begin{array}{c}0.449 \\
65\end{array}$ & $\begin{array}{c}0.4886 \\
9\end{array}$ & $\begin{array}{c}0.013 \\
09\end{array}$ & 2725 & 27 & 2655 & 33 & 2565 & $\begin{array}{l}5 \\
7\end{array}$ & $6.24 \%$ \\
\hline OUT\#07 & 242 & 426 & $\begin{array}{c}0.5 \\
7\end{array}$ & 0.07499 & $\begin{array}{c}0.003 \\
72\end{array}$ & $\begin{array}{c}1.3831 \\
8\end{array}$ & $\begin{array}{c}0.065 \\
01\end{array}$ & $\begin{array}{c}0.1337 \\
7\end{array}$ & $\begin{array}{c}0.002 \\
87\end{array}$ & 1068 & 60 & 882 & 28 & 809 & $\begin{array}{l}1 \\
6\end{array}$ & $9.02 \%$ \\
\hline OUT\#08 & 487 & 646 & 0.7 & 0.17785 & 0.001 & 12.359 & 0.126 & 0.5039 & 0.005 & 2633 & 8 & 2632 & 10 & 2631 & 2 & $0.08 \%$ \\
\hline
\end{tabular}




\begin{tabular}{|c|c|c|c|c|c|c|c|c|c|c|c|c|c|c|c|c|}
\hline & & & 5 & & 90 & 28 & 85 & 6 & 45 & & & & & & 3 & \\
\hline OUT\#09 & 104 & 271 & $\begin{array}{c}0.3 \\
9\end{array}$ & 0.05919 & $\begin{array}{c}0.003 \\
19\end{array}$ & $\begin{array}{c}0.6764 \\
9\end{array}$ & $\begin{array}{c}0.034 \\
82\end{array}$ & $\begin{array}{c}0.0828 \\
8\end{array}$ & $\begin{array}{c}0.001 \\
68\end{array}$ & 574 & 77 & 525 & 21 & 513 & $\begin{array}{l}1 \\
0\end{array}$ & $2.34 \%$ \\
\hline OUT\#10 & 63 & 99 & $\begin{array}{c}0.6 \\
4\end{array}$ & 0.12194 & $\begin{array}{c}0.003 \\
65\end{array}$ & $\begin{array}{c}5.7260 \\
8\end{array}$ & $\begin{array}{c}0.164 \\
05\end{array}$ & $\begin{array}{c}0.3405 \\
4\end{array}$ & $\begin{array}{c}0.006 \\
30\end{array}$ & 1985 & 26 & 1935 & 25 & 1889 & $\begin{array}{l}3 \\
0\end{array}$ & $5.08 \%$ \\
\hline OUT\#11 & 189 & 187 & $\begin{array}{c}1.0 \\
1\end{array}$ & 0.11810 & $\begin{array}{c}0.002 \\
69\end{array}$ & $\begin{array}{c}5.4946 \\
3\end{array}$ & $\begin{array}{c}0.119 \\
78\end{array}$ & $\begin{array}{c}0.3374 \\
4\end{array}$ & $\begin{array}{c}0.005 \\
05\end{array}$ & 1928 & 19 & 1900 & 19 & 1874 & $\begin{array}{l}2 \\
4\end{array}$ & $2.88 \%$ \\
\hline OUT\#12 & 221 & 248 & $\begin{array}{c}0.8 \\
9\end{array}$ & 0.06854 & $\begin{array}{c}0.004 \\
02\end{array}$ & $\begin{array}{c}0.8459 \\
4\end{array}$ & $\begin{array}{c}0.047 \\
03\end{array}$ & $\begin{array}{c}0.0895 \\
1\end{array}$ & $\begin{array}{c}0.002 \\
09\end{array}$ & 885 & & & 26 & 553 & $\begin{array}{l}1 \\
2\end{array}$ & $12.48 \%$ \\
\hline OUT\#13 & 77 & 144 & $\begin{array}{c}0.5 \\
4\end{array}$ & 0.06905 & $\begin{array}{c}0.012 \\
08\end{array}$ & $\begin{array}{c}0.7938 \\
6\end{array}$ & $\begin{array}{c}0.134 \\
86\end{array}$ & $\begin{array}{c}0.0833 \\
8\end{array}$ & $\begin{array}{c}0.003 \\
50\end{array}$ & 900 & $\begin{array}{c}39 \\
0\end{array}$ & 593 & 76 & 516 & $\begin{array}{l}2 \\
1\end{array}$ & $14.92 \%$ \\
\hline OUT\#14 & 100 & 108 & $\begin{array}{c}0.9 \\
2\end{array}$ & 0.07866 & $\begin{array}{c}0.007 \\
52\end{array}$ & $\begin{array}{c}1.0478 \\
0\end{array}$ & $\begin{array}{c}0.094 \\
78\end{array}$ & $\begin{array}{c}0.0966 \\
2\end{array}$ & $\begin{array}{c}0.003 \\
63\end{array}$ & 1164 & $\begin{array}{c}12 \\
0\end{array}$ & 728 & 47 & 595 & $\begin{array}{l}2 \\
1\end{array}$ & $22.35 \%$ \\
\hline OUT\#15 & 133 & 342 & $\begin{array}{c}0.3 \\
9\end{array}$ & 0.06470 & $\begin{array}{c}0.002 \\
92\end{array}$ & $\begin{array}{c}0.7862 \\
6\end{array}$ & $\begin{array}{c}0.033 \\
67\end{array}$ & $\begin{array}{c}0.0881 \\
4\end{array}$ & $\begin{array}{c}0.001 \\
61\end{array}$ & 765 & 59 & 589 & 19 & 545 & $\begin{array}{l}1 \\
0\end{array}$ & $8.07 \%$ \\
\hline OUT\#16 & 173 & 329 & $\begin{array}{c}0.5 \\
3\end{array}$ & 0.05740 & $\begin{array}{c}0.004 \\
38\end{array}$ & $\begin{array}{c}0.6332 \\
7\end{array}$ & $\begin{array}{c}0.046 \\
89\end{array}$ & $\begin{array}{c}0.0800 \\
1\end{array}$ & $\begin{array}{c}0.001 \\
47\end{array}$ & 507 & $\begin{array}{c}17 \\
3\end{array}$ & 498 & 29 & 496 & 9 & $0.40 \%$ \\
\hline OUT\#17 & 54 & 107 & $\begin{array}{c}0.5 \\
0\end{array}$ & 0.06786 & $\begin{array}{c}0.004 \\
21\end{array}$ & $\begin{array}{c}1.2763 \\
6\end{array}$ & $\begin{array}{c}0.075 \\
57\end{array}$ & $\begin{array}{c}0.1364 \\
3\end{array}$ & $\begin{array}{c}0.003 \\
40\end{array}$ & 864 & 81 & 835 & 34 & 824 & $\begin{array}{l}1 \\
9\end{array}$ & $1.33 \%$ \\
\hline OUT\#18 & 54 & 71 & $\begin{array}{c}0.7 \\
7\end{array}$ & 0.10532 & $\begin{array}{c}0.007 \\
77\end{array}$ & $\begin{array}{c}4.0942 \\
2\end{array}$ & $\begin{array}{c}0.283 \\
60\end{array}$ & $\begin{array}{c}0.2819 \\
4\end{array}$ & $\begin{array}{c}0.007 \\
18\end{array}$ & 1720 & $\begin{array}{c}14 \\
0\end{array}$ & 1653 & 57 & 1601 & $\begin{array}{l}3 \\
6\end{array}$ & $7.43 \%$ \\
\hline OUT\#19 & 72 & 73 & $\begin{array}{c}0.9 \\
8\end{array}$ & 0.06463 & $\begin{array}{c}0.006 \\
57\end{array}$ & $\begin{array}{c}0.7848 \\
1\end{array}$ & $\begin{array}{c}0.076 \\
16\end{array}$ & $\begin{array}{c}0.0880 \\
9\end{array}$ & $\begin{array}{c}0.003 \\
19\end{array}$ & 762 & $\begin{array}{c}14 \\
4\end{array}$ & 588 & 43 & 544 & $\begin{array}{l}1 \\
9\end{array}$ & $8.09 \%$ \\
\hline OUT\#20 & 111 & 231 & $\begin{array}{c}0.4 \\
8\end{array}$ & 0 & $\begin{array}{c}0.003 \\
61\end{array}$ & $\begin{array}{c}0.8366 \\
9\end{array}$ & $\begin{array}{c}0.042 \\
19\end{array}$ & $\begin{array}{c}0.0892 \\
3\end{array}$ & $\begin{array}{c}0.001 \\
89\end{array}$ & 869 & 69 & 617 & 23 & 551 & $\begin{array}{l}1 \\
1\end{array}$ & $11.98 \%$ \\
\hline OUT\#21 & 112 & 178 & $\begin{array}{c}0.6 \\
3\end{array}$ & 0.11986 & $\begin{array}{c}0.002 \\
73\end{array}$ & $\begin{array}{c}5.4659 \\
8\end{array}$ & $\begin{array}{c}0.118 \\
64\end{array}$ & $\begin{array}{c}0.3308 \\
1\end{array}$ & $\begin{array}{c}0.004 \\
95\end{array}$ & 1954 & 19 & 1895 & 19 & 1842 & $\begin{array}{l}2 \\
4\end{array}$ & $6.08 \%$ \\
\hline OUT\#22 & 92 & 107 & $\begin{array}{c}0.8 \\
5\end{array}$ & 0.11334 & $\begin{array}{c}0.003 \\
56\end{array}$ & $\begin{array}{c}4.6623 \\
4\end{array}$ & $\begin{array}{c}0.139 \\
58\end{array}$ & $\begin{array}{c}0.2984 \\
0\end{array}$ & $\begin{array}{c}0.005 \\
51\end{array}$ & 1854 & 29 & 1761 & 25 & 1683 & $\begin{array}{l}2 \\
7\end{array}$ & $10.16 \%$ \\
\hline OUT\#23 & 60 & 114 & $\begin{array}{c}0.5 \\
3\end{array}$ & 0.05691 & $\begin{array}{c}0.004 \\
46\end{array}$ & $\begin{array}{c}0.7449 \\
6\end{array}$ & $\begin{array}{c}0.056 \\
00\end{array}$ & $\begin{array}{c}0.0949 \\
6\end{array}$ & $\begin{array}{c}0.002 \\
61\end{array}$ & 488 & $\begin{array}{c}11 \\
7\end{array}$ & 565 & 33 & 585 & $\begin{array}{l}1 \\
5\end{array}$ & $-3.42 \%$ \\
\hline OUT\#24 & 48 & 54 & $\begin{array}{c}0.8 \\
8\end{array}$ & 0.04991 & $\begin{array}{c}0.013 \\
47\end{array}$ & $\begin{array}{c}0.5544 \\
4\end{array}$ & $\begin{array}{c}0.146 \\
82\end{array}$ & $\begin{array}{c}0.0805 \\
7\end{array}$ & $\begin{array}{c}0.004 \\
16\end{array}$ & 191 & $\begin{array}{c}47 \\
0\end{array}$ & 448 & 96 & 500 & $\begin{array}{l}2 \\
5\end{array}$ & $-10.40 \%$ \\
\hline OUT\#25 & 157 & 279 & $\begin{array}{c}0.5 \\
6\end{array}$ & 0.06676 & $\begin{array}{c}0.002 \\
61\end{array}$ & $\begin{array}{c}1.2157 \\
9\end{array}$ & $\begin{array}{c}0.045 \\
36\end{array}$ & $\begin{array}{c}0.1321 \\
2\end{array}$ & $\begin{array}{c}0.002 \\
26\end{array}$ & 830 & 49 & 808 & 21 & 800 & $\begin{array}{l}1 \\
3\end{array}$ & $1.00 \%$ \\
\hline
\end{tabular}




\begin{tabular}{|c|c|c|c|c|c|c|c|c|c|c|c|c|c|c|c|c|}
\hline OUT\#26 & 86 & 145 & $\begin{array}{c}0.6 \\
0\end{array}$ & 0.06484 & $\begin{array}{c}0.004 \\
94\end{array}$ & $\begin{array}{c}0.8492 \\
1\end{array}$ & $\begin{array}{c}0.061 \\
58\end{array}$ & $\begin{array}{c}0.0950 \\
2\end{array}$ & $\begin{array}{c}0.002 \\
73\end{array}$ & 769 & $\begin{array}{c}10 \\
4\end{array}$ & 624 & 34 & 585 & $\begin{array}{l}1 \\
6\end{array}$ & $6.67 \%$ \\
\hline OUT\#27 & 145 & 71 & $\begin{array}{c}2.0 \\
5\end{array}$ & 0.13101 & $\begin{array}{c}0.006 \\
12\end{array}$ & $\begin{array}{c}5.6786 \\
6\end{array}$ & $\begin{array}{c}0.252 \\
06\end{array}$ & $\begin{array}{c}0.3144 \\
4\end{array}$ & $\begin{array}{c}0.008 \\
61\end{array}$ & 2112 & 41 & 1928 & 38 & 1763 & $\begin{array}{l}4 \\
2\end{array}$ & $19.80 \%$ \\
\hline OUT\#28 & 432 & 622 & $\begin{array}{c}0.6 \\
9\end{array}$ & 0.06070 & $\begin{array}{c}0.003 \\
46\end{array}$ & $\begin{array}{c}0.7319 \\
7\end{array}$ & $\begin{array}{c}0.040 \\
24\end{array}$ & $\begin{array}{c}0.0874 \\
6\end{array}$ & $\begin{array}{c}0.001 \\
29\end{array}$ & 628 & $\begin{array}{c}12 \\
6\end{array}$ & 558 & 24 & 541 & 8 & $3.14 \%$ \\
\hline OUT\#29 & 70 & 32 & $\begin{array}{c}2.1 \\
6\end{array}$ & 0.05019 & $\begin{array}{c}0.009 \\
33\end{array}$ & $\begin{array}{c}0.6904 \\
2\end{array}$ & $\begin{array}{c}0.124 \\
44\end{array}$ & $\begin{array}{c}0.0998 \\
0\end{array}$ & $\begin{array}{c}0.005 \\
44\end{array}$ & 204 & & & 75 & 613 & $\begin{array}{l}3 \\
2\end{array}$ & $-13.05 \%$ \\
\hline OUT\#30 & 46 & 324 & $\begin{array}{c}0.1 \\
4\end{array}$ & 0.05944 & $\begin{array}{c}0.002 \\
87\end{array}$ & $\begin{array}{c}0.7562 \\
2\end{array}$ & $\begin{array}{c}0.034 \\
87\end{array}$ & $\begin{array}{c}0.0923 \\
0\end{array}$ & $\begin{array}{c}0.001 \\
74\end{array}$ & 5 & & 572 & 20 & 569 & $\begin{array}{l}1 \\
0\end{array}$ & $0.53 \%$ \\
\hline OUT\#31 & 183 & 350 & $\begin{array}{c}0.5 \\
2\end{array}$ & 0.06282 & $\begin{array}{c}0.002 \\
82\end{array}$ & $\begin{array}{c}0.9311 \\
8\end{array}$ & $\begin{array}{c}0.039 \\
82\end{array}$ & $\begin{array}{c}0.1075 \\
5\end{array}$ & $\begin{array}{c}0.001 \\
97\end{array}$ & 702 & 60 & 668 & 21 & 659 & $\begin{array}{l}1 \\
1\end{array}$ & $1.37 \%$ \\
\hline OUT\#32 & 229 & 179 & $\begin{array}{c}1.2 \\
8\end{array}$ & 0.22239 & $\begin{array}{c}0.003 \\
32\end{array}$ & $\begin{array}{c}17.568 \\
58\end{array}$ & $\begin{array}{c}0.258 \\
27\end{array}$ & $\begin{array}{c}0.5731 \\
7\end{array}$ & $\begin{array}{c}0.007 \\
99\end{array}$ & 2998 & 11 & 2966 & 14 & 2921 & $\begin{array}{l}3 \\
3\end{array}$ & $2.64 \%$ \\
\hline OUT\#33 & 81 & 79 & $\begin{array}{c}1.0 \\
3\end{array}$ & 0.07687 & $\begin{array}{c}0.008 \\
26\end{array}$ & $\begin{array}{c}1.1186 \\
5\end{array}$ & $\begin{array}{c}0.113 \\
74\end{array}$ & $\begin{array}{c}0.1055 \\
9\end{array}$ & $\begin{array}{c}0.004 \\
50\end{array}$ & 1118 & $\begin{array}{c}13 \\
5\end{array}$ & 762 & 55 & 647 & 5 & $17.77 \%$ \\
\hline OUT\#34 & 67 & 196 & $\begin{array}{c}0.3 \\
4\end{array}$ & 0.07149 & $\begin{array}{c}0.003 \\
95\end{array}$ & $\begin{array}{c}1.1329 \\
1\end{array}$ & $\begin{array}{c}0.059 \\
34\end{array}$ & $\begin{array}{c}0.1149 \\
8\end{array}$ & $\begin{array}{c}0.002 \\
63\end{array}$ & 971 & 70 & 769 & 28 & 702 & $\begin{array}{l}1 \\
5\end{array}$ & $9.54 \%$ \\
\hline OUT\#35 & 64 & 92 & $\begin{array}{c}0.6 \\
9\end{array}$ & 0.11756 & $\begin{array}{c}0.004 \\
89\end{array}$ & $\begin{array}{c}5.4351 \\
9\end{array}$ & $\begin{array}{c}0.216 \\
35\end{array}$ & $\begin{array}{c}0.3354 \\
6\end{array}$ & $\begin{array}{c}0.008 \\
10\end{array}$ & 1919 & 38 & 1890 & 34 & 1865 & $\begin{array}{l}3 \\
9\end{array}$ & $2.90 \%$ \\
\hline OUT\#36 & 45 & 156 & $\begin{array}{c}0.2 \\
9\end{array}$ & 0.21104 & $\begin{array}{c}0.004 \\
11\end{array}$ & $\begin{array}{c}16.367 \\
23\end{array}$ & $\begin{array}{c}0.314 \\
41\end{array}$ & $\begin{array}{c}0.5627 \\
4\end{array}$ & $\begin{array}{c}0.009 \\
42\end{array}$ & 2914 & 14 & 2898 & 18 & 2878 & $\begin{array}{l}3 \\
9\end{array}$ & $1.25 \%$ \\
\hline OUT\#37 & 46 & 161 & $\begin{array}{c}0.2 \\
8\end{array}$ & 0.12590 & $\begin{array}{c}0.003 \\
90\end{array}$ & $\begin{array}{c}5.5708 \\
6\end{array}$ & $\begin{array}{c}0.164 \\
46\end{array}$ & $\begin{array}{c}0.3210 \\
8\end{array}$ & $\begin{array}{c}0.006 \\
15\end{array}$ & 2041 & 27 & 1912 & 25 & 1795 & $\begin{array}{l}3 \\
0\end{array}$ & $13.70 \%$ \\
\hline OUT\#38 & 96 & 131 & $\begin{array}{c}0.7 \\
3\end{array}$ & 0.06906 & $\begin{array}{c}0.009 \\
23\end{array}$ & $\begin{array}{c}0.8230 \\
3\end{array}$ & $\begin{array}{c}0.106 \\
86\end{array}$ & $\begin{array}{c}0.0864 \\
4\end{array}$ & $\begin{array}{c}0.002 \\
72\end{array}$ & 901 & $\begin{array}{c}29 \\
2\end{array}$ & 610 & 60 & 534 & $\begin{array}{l}1 \\
6\end{array}$ & $14.23 \%$ \\
\hline OUT\#39 & 77 & 28 & $\begin{array}{c}2.8 \\
0\end{array}$ & 0.12691 & $\begin{array}{c}0.007 \\
13\end{array}$ & $\begin{array}{c}5.6552 \\
0\end{array}$ & $\begin{array}{c}0.302 \\
85\end{array}$ & $\begin{array}{c}0.3233 \\
5\end{array}$ & $\begin{array}{c}0.010 \\
47\end{array}$ & 2056 & 51 & 1925 & 46 & 1806 & $\begin{array}{l}5 \\
1\end{array}$ & $13.84 \%$ \\
\hline OUT\#40 & 56 & 76 & $\begin{array}{c}0.7 \\
3\end{array}$ & 0.06094 & $\begin{array}{c}0.005 \\
84\end{array}$ & $\begin{array}{c}0.9431 \\
4\end{array}$ & $\begin{array}{c}0.086 \\
50\end{array}$ & $\begin{array}{c}0.1123 \\
0\end{array}$ & $\begin{array}{c}0.003 \\
91\end{array}$ & 637 & $\begin{array}{c}13 \\
8\end{array}$ & 675 & 45 & 686 & $\begin{array}{l}2 \\
3\end{array}$ & $-1.60 \%$ \\
\hline OUT\#41 & 165 & 261 & $\begin{array}{c}0.6 \\
3\end{array}$ & 0.06314 & $\begin{array}{c}0.002 \\
97\end{array}$ & $\begin{array}{c}0.8883 \\
5\end{array}$ & $\begin{array}{c}0.039 \\
87\end{array}$ & $\begin{array}{c}0.1021 \\
0\end{array}$ & $\begin{array}{c}0.001 \\
94\end{array}$ & 713 & 63 & 645 & 21 & 627 & $\begin{array}{l}1 \\
1\end{array}$ & $2.87 \%$ \\
\hline OUT\#42 & 63 & 90 & $\begin{array}{c}0.7 \\
0\end{array}$ & 0.11900 & $\begin{array}{c}0.003 \\
58\end{array}$ & $\begin{array}{c}5.4870 \\
6\end{array}$ & $\begin{array}{c}0.157 \\
64\end{array}$ & $\begin{array}{c}0.3346 \\
1\end{array}$ & $\begin{array}{c}0.006 \\
18\end{array}$ & 1941 & 27 & 1899 & 25 & 1861 & $\begin{array}{l}3 \\
0\end{array}$ & $4.30 \%$ \\
\hline OUT\#43 & 39 & 50 & 0.7 & 0.10418 & 0.005 & 4.4940 & 0.217 & 0.3130 & 0.008 & 1700 & 50 & 1730 & 40 & 1756 & 4 & $-3.19 \%$ \\
\hline
\end{tabular}




\begin{tabular}{|c|c|c|c|c|c|c|c|c|c|c|c|c|c|c|c|c|}
\hline & & & 8 & & 25 & 9 & 20 & 6 & 46 & & & & & & 2 & \\
\hline OUT\#44 & 105 & 100 & $\begin{array}{c}1.0 \\
5\end{array}$ & 0.06157 & $\begin{array}{c}0.004 \\
96\end{array}$ & $\begin{array}{c}0.8008 \\
1\end{array}$ & $\begin{array}{c}0.061 \\
65\end{array}$ & $\begin{array}{c}0.0943 \\
8\end{array}$ & $\begin{array}{c}0.002 \\
77\end{array}$ & 659 & $\begin{array}{c}11 \\
5\end{array}$ & 597 & 35 & 581 & $\begin{array}{l}1 \\
6\end{array}$ & $2.75 \%$ \\
\hline OUT\#45 & 100 & 286 & $\begin{array}{c}0.3 \\
5\end{array}$ & 0.10643 & $\begin{array}{c}0.002 \\
27\end{array}$ & $\begin{array}{c}4.4361 \\
2\end{array}$ & $\begin{array}{c}0.090 \\
12\end{array}$ & $\begin{array}{c}0.3024 \\
9\end{array}$ & $\begin{array}{c}0.004 \\
18\end{array}$ & 1739 & 19 & 1719 & 17 & 1704 & $\begin{array}{l}2 \\
1\end{array}$ & $2.05 \%$ \\
\hline OUT\#46 & 73 & 92 & $\begin{array}{c}0.7 \\
9\end{array}$ & 0.07070 & $\begin{array}{c}0.009 \\
22\end{array}$ & $\begin{array}{c}0.9352 \\
9\end{array}$ & $\begin{array}{c}0.115 \\
97\end{array}$ & $\begin{array}{c}0.0960 \\
1\end{array}$ & $\begin{array}{c}0.004 \\
65\end{array}$ & 949 & 17 & & 61 & 591 & $\begin{array}{l}2 \\
7\end{array}$ & $13.37 \%$ \\
\hline OUT\#47 & 27 & 37 & $\begin{array}{c}0.7 \\
4\end{array}$ & 0.11755 & $\begin{array}{c}0.015 \\
02\end{array}$ & $\begin{array}{c}4.0689 \\
3\end{array}$ & $\begin{array}{c}0.486 \\
00\end{array}$ & $\begin{array}{c}0.2510 \\
4\end{array}$ & $\begin{array}{c}0.011 \\
39\end{array}$ & 01 & & & 97 & 1444 & $\begin{array}{l}5 \\
9\end{array}$ & $32.89 \%$ \\
\hline OUT\#48 & 194 & 269 & $\begin{array}{c}0.7 \\
2\end{array}$ & 0.05782 & $\begin{array}{c}0.002 \\
86\end{array}$ & $\begin{array}{c}0.7741 \\
3\end{array}$ & $\begin{array}{c}0.036 \\
65\end{array}$ & $\begin{array}{c}0.0971 \\
7\end{array}$ & $\begin{array}{c}0.001 \\
86\end{array}$ & 523 & 70 & 582 & 21 & 598 & $\begin{array}{l}1 \\
1\end{array}$ & $-2.68 \%$ \\
\hline OUT\#49 & 258 & 655 & $\begin{array}{c}0.3 \\
9\end{array}$ & 0.10485 & $\begin{array}{c}0.004 \\
10\end{array}$ & $\begin{array}{c}1.2009 \\
5\end{array}$ & $\begin{array}{c}0.043 \\
31\end{array}$ & $\begin{array}{c}0.0831 \\
3\end{array}$ & $\begin{array}{c}0.001 \\
61\end{array}$ & 1712 & 38 & 801 & 20 & 515 & $\begin{array}{l}1 \\
0\end{array}$ & $55.53 \%$ \\
\hline OUT\#50 & 68 & 101 & $\begin{array}{c}0.6 \\
7\end{array}$ & 0.10465 & $\begin{array}{c}0.008 \\
05\end{array}$ & $\begin{array}{c}4.4019 \\
7\end{array}$ & $\begin{array}{c}0.316 \\
21\end{array}$ & $\begin{array}{c}0.3050 \\
9\end{array}$ & $\begin{array}{c}0.008 \\
37\end{array}$ & 1708 & $\begin{array}{c}14 \\
6\end{array}$ & 1713 & 59 & 1716 & $\begin{array}{l}4 \\
1\end{array}$ & $-0.47 \%$ \\
\hline OUT\#51 & 284 & 248 & $\begin{array}{c}1.1 \\
5\end{array}$ & 0.06388 & $\begin{array}{c}0.002 \\
74\end{array}$ & $\begin{array}{c}1.0896 \\
8\end{array}$ & $\begin{array}{c}0.044 \\
57\end{array}$ & $\begin{array}{c}0.1238 \\
2\end{array}$ & $\begin{array}{c}0.002 \\
23\end{array}$ & 738 & 56 & 748 & 22 & 753 & $\begin{array}{l}1 \\
3\end{array}$ & $-0.66 \%$ \\
\hline OUT\#52 & 277 & 274 & $\begin{array}{c}1.0 \\
1\end{array}$ & 0.13142 & $\begin{array}{c}0.008 \\
98\end{array}$ & $\begin{array}{c}5.5943 \\
7\end{array}$ & $\begin{array}{c}0.360 \\
70\end{array}$ & $\begin{array}{c}0.3087 \\
3\end{array}$ & $\begin{array}{c}0.006 \\
97\end{array}$ & 2117 & $\begin{array}{c}12 \\
3\end{array}$ & 1915 & 56 & 1734 & $\begin{array}{l}3 \\
4\end{array}$ & $22.09 \%$ \\
\hline OUT\#53 & 111 & 221 & $\begin{array}{c}0.5 \\
0\end{array}$ & 0.13582 & $\begin{array}{c}0.002 \\
64\end{array}$ & $\begin{array}{c}7.1681 \\
6\end{array}$ & $\begin{array}{c}0.133 \\
53\end{array}$ & $\begin{array}{c}0.3831 \\
1\end{array}$ & $\begin{array}{c}0.005 \\
43\end{array}$ & 2175 & 15 & 2133 & 17 & 2091 & $\begin{array}{l}2 \\
5\end{array}$ & $4.02 \%$ \\
\hline OUT\#54 & 102 & 154 & $\begin{array}{c}0.6 \\
7\end{array}$ & 0.06809 & $\begin{array}{c}0.003 \\
39\end{array}$ & $\begin{array}{c}1.3101 \\
9\end{array}$ & $\begin{array}{c}0.062 \\
20\end{array}$ & $\begin{array}{c}0.1396 \\
8\end{array}$ & $\begin{array}{c}0.002 \\
94\end{array}$ & 871 & 64 & 850 & 27 & 843 & $\begin{array}{l}1 \\
7\end{array}$ & $0.83 \%$ \\
\hline OUT\#55 & 102 & 77 & $\begin{array}{c}1.3 \\
3\end{array}$ & 0.06563 & $\begin{array}{c}0.012 \\
51\end{array}$ & $\begin{array}{c}0.8409 \\
9\end{array}$ & $\begin{array}{c}0.157 \\
04\end{array}$ & $\begin{array}{c}0.0929 \\
4\end{array}$ & $\begin{array}{c}0.003 \\
60\end{array}$ & 794 & $\begin{array}{c}42 \\
8\end{array}$ & 620 & 87 & 573 & $\begin{array}{l}2 \\
1\end{array}$ & $8.20 \%$ \\
\hline OUT\#56 & 69 & 131 & $\begin{array}{c}0.5 \\
3\end{array}$ & 0.05769 & $\begin{array}{c}0.004 \\
12\end{array}$ & $\begin{array}{c}0.8280 \\
7\end{array}$ & $\begin{array}{c}0.056 \\
59\end{array}$ & $\begin{array}{c}0.1041 \\
9\end{array}$ & $\begin{array}{c}0.002 \\
69\end{array}$ & 518 & $\begin{array}{c}10 \\
5\end{array}$ & 613 & 31 & 639 & $\begin{array}{l}1 \\
6\end{array}$ & $-4.07 \%$ \\
\hline OUT\#57 & 78 & 70 & $\begin{array}{c}1.1 \\
1\end{array}$ & 0.11860 & $\begin{array}{c}0.004 \\
53\end{array}$ & $\begin{array}{c}5.3964 \\
1\end{array}$ & $\begin{array}{c}0.197 \\
05\end{array}$ & $\begin{array}{c}0.3303 \\
2\end{array}$ & $\begin{array}{c}0.007 \\
43\end{array}$ & 1935 & 35 & 1884 & 31 & 1840 & $\begin{array}{l}3 \\
6\end{array}$ & $5.16 \%$ \\
\hline OUT\#58 & 86 & 78 & $\begin{array}{c}1.1 \\
1\end{array}$ & 0.05727 & $\begin{array}{c}0.005 \\
68\end{array}$ & $\begin{array}{c}0.7502 \\
2\end{array}$ & $\begin{array}{c}0.071 \\
45\end{array}$ & $\begin{array}{c}0.0951 \\
1\end{array}$ & $\begin{array}{c}0.003 \\
17\end{array}$ & 502 & $\begin{array}{c}15 \\
2\end{array}$ & 568 & 41 & 586 & $\begin{array}{l}1 \\
9\end{array}$ & $-3.07 \%$ \\
\hline OUT\#59 & 54 & 135 & $\begin{array}{c}0.4 \\
0\end{array}$ & 0.12899 & $\begin{array}{c}0.004 \\
79\end{array}$ & $\begin{array}{c}5.6651 \\
5\end{array}$ & $\begin{array}{c}0.184 \\
63\end{array}$ & $\begin{array}{c}0.3185 \\
3\end{array}$ & $\begin{array}{c}0.005 \\
65\end{array}$ & 2084 & 67 & 1926 & 28 & 1783 & $\begin{array}{l}2 \\
8\end{array}$ & $16.88 \%$ \\
\hline OUT\#60 & 278 & 226 & $\begin{array}{c}1.2 \\
3\end{array}$ & 0.05843 & $\begin{array}{c}0.003 \\
17\end{array}$ & $\begin{array}{c}0.7517 \\
4\end{array}$ & $\begin{array}{c}0.038 \\
95\end{array}$ & $\begin{array}{c}0.0934 \\
0\end{array}$ & $\begin{array}{c}0.001 \\
93\end{array}$ & 546 & 77 & 569 & 23 & 576 & $\begin{array}{l}1 \\
1\end{array}$ & $-1.22 \%$ \\
\hline
\end{tabular}




\begin{tabular}{|c|c|c|c|c|c|c|c|c|c|c|c|c|c|c|c|c|}
\hline OUT\#61 & 152 & 345 & $\begin{array}{c}0.4 \\
4\end{array}$ & 0.13741 & $\begin{array}{c}0.002 \\
45\end{array}$ & $\begin{array}{c}7.4628 \\
0\end{array}$ & $\begin{array}{c}0.127 \\
70\end{array}$ & $\begin{array}{c}0.3943 \\
2\end{array}$ & $\begin{array}{c}0.005 \\
32\end{array}$ & 2195 & 14 & 2169 & 15 & 2143 & $\begin{array}{l}2 \\
5\end{array}$ & $2.43 \%$ \\
\hline OUT\#62 & 261 & 385 & $\begin{array}{c}0.6 \\
8\end{array}$ & 0.15211 & $\begin{array}{c}0.002 \\
36\end{array}$ & $\begin{array}{c}8.4804 \\
7\end{array}$ & $\begin{array}{c}0.126 \\
41\end{array}$ & $\begin{array}{c}0.4047 \\
9\end{array}$ & $\begin{array}{c}0.005 \\
15\end{array}$ & 2370 & 11 & 2284 & 14 & 2191 & $\begin{array}{l}2 \\
4\end{array}$ & $8.17 \%$ \\
\hline OUT\#63 & 72 & 132 & $\begin{array}{c}0.5 \\
5\end{array}$ & 0.06137 & $\begin{array}{c}0.004 \\
40\end{array}$ & $\begin{array}{c}0.7721 \\
2\end{array}$ & $\begin{array}{c}0.052 \\
78\end{array}$ & $\begin{array}{c}0.0913 \\
5\end{array}$ & $\begin{array}{c}0.002 \\
46\end{array}$ & 652 & $\begin{array}{c}10 \\
0\end{array}$ & 581 & 30 & 564 & 1 & $3.01 \%$ \\
\hline OUT\#64 & 189 & 211 & $\begin{array}{c}0.9 \\
0\end{array}$ & 0.06223 & $\begin{array}{c}0.003 \\
26\end{array}$ & $\begin{array}{c}0.7913 \\
5\end{array}$ & $\begin{array}{c}0.039 \\
48\end{array}$ & $\begin{array}{c}0.0923 \\
3\end{array}$ & $\begin{array}{c}0.001 \\
90\end{array}$ & 682 & & & 22 & 569 & t & $4.04 \%$ \\
\hline OUT\#65 & 75 & 110 & $\begin{array}{c}0.6 \\
8\end{array}$ & 0.13219 & $\begin{array}{c}0.003 \\
47\end{array}$ & $\begin{array}{c}7.4321 \\
8\end{array}$ & $\begin{array}{c}0.188 \\
01\end{array}$ & $\begin{array}{c}0.4082 \\
6\end{array}$ & $\begin{array}{c}0.007 \\
21\end{array}$ & 212 & & 2165 & 23 & 2207 & $\begin{array}{l}3 \\
3\end{array}$ & $-3.62 \%$ \\
\hline OUT\#66 & 85 & 132 & $\begin{array}{c}0.6 \\
5\end{array}$ & 0.06987 & $\begin{array}{c}0.003 \\
61\end{array}$ & $\begin{array}{c}1.4178 \\
4\end{array}$ & $\begin{array}{c}0.069 \\
78\end{array}$ & $\begin{array}{c}0.1473 \\
4\end{array}$ & $\begin{array}{c}0.003 \\
21\end{array}$ & 925 & 65 & 896 & 29 & 886 & $\begin{array}{l}1 \\
8\end{array}$ & $1.13 \%$ \\
\hline OUT\#67 & 139 & 247 & $\begin{array}{c}0.5 \\
6\end{array}$ & 0.07537 & $\begin{array}{c}0.003 \\
49\end{array}$ & $\begin{array}{c}1.3407 \\
6\end{array}$ & $\begin{array}{c}0.058 \\
89\end{array}$ & $\begin{array}{c}0.1291 \\
7\end{array}$ & $\begin{array}{c}0.002 \\
63\end{array}$ & 1078 & 56 & 864 & 26 & 783 & ) & $10.34 \%$ \\
\hline OUT\#68 & 100 & 638 & $\begin{array}{c}0.1 \\
6\end{array}$ & 0.08231 & $\begin{array}{c}0.002 \\
56\end{array}$ & $\begin{array}{c}1.5570 \\
0\end{array}$ & $\begin{array}{c}0.043 \\
67\end{array}$ & $\begin{array}{c}0.1371 \\
9\end{array}$ & $\begin{array}{c}0.001 \\
85\end{array}$ & 1253 & 62 & 953 & 17 & 829 & $\begin{array}{l}1 \\
0\end{array}$ & $14.96 \%$ \\
\hline OUT\#69 & 142 & 166 & $\begin{array}{c}0.8 \\
6\end{array}$ & 0.06509 & $\begin{array}{c}0.003 \\
74\end{array}$ & $\begin{array}{c}0.9238 \\
9\end{array}$ & $\begin{array}{c}0.050 \\
51\end{array}$ & $\begin{array}{c}0.1030 \\
7\end{array}$ & $\begin{array}{c}0.002 \\
34\end{array}$ & 777 & 77 & 664 & 27 & 632 & $\begin{array}{l}1 \\
4\end{array}$ & $5.06 \%$ \\
\hline OUT\#70 & 178 & 568 & $\begin{array}{c}0.3 \\
1\end{array}$ & 0.05870 & $\begin{array}{c}0.002 \\
17\end{array}$ & 0.6369 & $\begin{array}{c}0.022 \\
47\end{array}$ & $\begin{array}{c}0.0788 \\
1\end{array}$ & $\begin{array}{c}0.001 \\
22\end{array}$ & 556 & 50 & 500 & 14 & 489 & 7 & $2.25 \%$ \\
\hline OUT\#71 & 70 & 90 & $\begin{array}{c}0.7 \\
8\end{array}$ & 0.10720 & $\begin{array}{c}0.004 \\
11\end{array}$ & $\frac{9867}{6}$ & $\begin{array}{c}0.145 \\
17\end{array}$ & $\begin{array}{c}0.2701 \\
0\end{array}$ & $\begin{array}{c}0.005 \\
74\end{array}$ & 1752 & 37 & 1632 & 30 & 1541 & $\begin{array}{l}2 \\
9\end{array}$ & $13.69 \%$ \\
\hline OUT\#72 & 302 & 367 & $\begin{array}{c}0.8 \\
2\end{array}$ & 0.06324 & $\begin{array}{c}0.002 \\
35\end{array}$ & $\begin{array}{c}0.9241 \\
4\end{array}$ & $\begin{array}{c}0.032 \\
69\end{array}$ & $\begin{array}{c}0.1061 \\
4\end{array}$ & $\begin{array}{c}0.001 \\
71\end{array}$ & 716 & 48 & 665 & 17 & 650 & $\begin{array}{l}1 \\
0\end{array}$ & $2.31 \%$ \\
\hline OUT\#73 & 35 & 39 & $\begin{array}{c}0.9 \\
0\end{array}$ & 0.12664 & $\begin{array}{c}0.010 \\
49\end{array}$ & $\begin{array}{c}4.2292 \\
3\end{array}$ & $\begin{array}{c}0.329 \\
31\end{array}$ & $\begin{array}{c}0.2425 \\
4\end{array}$ & $\begin{array}{c}0.010 \\
67\end{array}$ & 2052 & 77 & 1680 & 64 & 1400 & $\begin{array}{l}5 \\
5\end{array}$ & $46.57 \%$ \\
\hline OUT\#74 & 252 & 375 & $\begin{array}{c}0.6 \\
7\end{array}$ & 0.05957 & $\begin{array}{c}0.004 \\
89\end{array}$ & $\begin{array}{c}0.7238 \\
8\end{array}$ & $\begin{array}{c}0.057 \\
78\end{array}$ & $\begin{array}{c}0.0881 \\
3\end{array}$ & $\begin{array}{c}0.001 \\
67\end{array}$ & 588 & $\begin{array}{c}18 \\
4\end{array}$ & 553 & 34 & 544 & $\begin{array}{l}1 \\
0\end{array}$ & $1.65 \%$ \\
\hline OUT\#75 & 146 & 176 & $\begin{array}{c}0.8 \\
3\end{array}$ & 0.17771 & $\begin{array}{c}0.003 \\
70\end{array}$ & $\begin{array}{c}11.822 \\
83\end{array}$ & $\begin{array}{c}0.239 \\
11\end{array}$ & $\begin{array}{c}0.4832 \\
1\end{array}$ & $\begin{array}{c}0.007 \\
94\end{array}$ & 2632 & 15 & 2590 & 19 & 2541 & $\begin{array}{l}3 \\
5\end{array}$ & $3.58 \%$ \\
\hline OUT\#76 & 22 & 127 & $\begin{array}{c}0.1 \\
7\end{array}$ & 0.05226 & $\begin{array}{c}0.013 \\
30\end{array}$ & $\begin{array}{c}0.3423 \\
5\end{array}$ & $\begin{array}{c}0.085 \\
50\end{array}$ & $\begin{array}{c}0.0475 \\
1\end{array}$ & $\begin{array}{c}0.002 \\
30\end{array}$ & 297 & $\begin{array}{c}46 \\
6\end{array}$ & 299 & 65 & 299 & $\begin{array}{l}1 \\
4\end{array}$ & $0.00 \%$ \\
\hline OUT\#77 & 237 & 393 & $\begin{array}{c}0.6 \\
0\end{array}$ & 0.06023 & $\begin{array}{c}0.003 \\
85\end{array}$ & $\begin{array}{c}0.8500 \\
4\end{array}$ & $\begin{array}{c}0.052 \\
36\end{array}$ & $\begin{array}{c}0.1023 \\
7\end{array}$ & $\begin{array}{c}0.001 \\
73\end{array}$ & 612 & $\begin{array}{c}14 \\
2\end{array}$ & 625 & 29 & 628 & $\begin{array}{l}1 \\
0\end{array}$ & $-0.48 \%$ \\
\hline OUT\#78 & 309 & 195 & 1.5 & 0.06300 & 0.003 & 0.8895 & 0.044 & 0.1025 & 0.002 & 708 & 70 & 646 & 24 & 629 & 1 & $2.70 \%$ \\
\hline
\end{tabular}




\begin{tabular}{|c|c|c|c|c|c|c|c|c|c|c|c|c|c|c|c|c|}
\hline & & & 8 & & 28 & 8 & 05 & 7 & 13 & & & & & & 2 & \\
\hline OUT\#79 & 365 & 302 & $\begin{array}{c}1.2 \\
1\end{array}$ & 0.08200 & $\begin{array}{c}0.003 \\
55\end{array}$ & $\begin{array}{c}1.0864 \\
6\end{array}$ & $\begin{array}{c}0.044 \\
22\end{array}$ & $\begin{array}{c}0.0962 \\
5\end{array}$ & $\begin{array}{c}0.001 \\
88\end{array}$ & 1246 & 49 & 747 & 22 & 592 & $\begin{array}{l}1 \\
1\end{array}$ & $26.18 \%$ \\
\hline OUT\#80 & 21 & 30 & $\begin{array}{c}0.6 \\
8\end{array}$ & 0.12079 & $\begin{array}{c}0.007 \\
88\end{array}$ & $\begin{array}{c}4.9962 \\
6\end{array}$ & $\begin{array}{c}0.309 \\
58\end{array}$ & $\begin{array}{c}0.3004 \\
5\end{array}$ & $\begin{array}{c}0.010 \\
89\end{array}$ & 1968 & 61 & 1819 & 52 & 1694 & $\begin{array}{l}5 \\
4\end{array}$ & $16.17 \%$ \\
\hline OUT\#81 & 109 & 124 & $\begin{array}{c}0.8 \\
8\end{array}$ & 0.11652 & $\begin{array}{c}0.003 \\
28\end{array}$ & $\begin{array}{c}4.9981 \\
3\end{array}$ & $\begin{array}{c}0.133 \\
96\end{array}$ & $\begin{array}{c}0.3116 \\
1\end{array}$ & $\begin{array}{c}0.005 \\
38\end{array}$ & 1904 & 25 & 1810 & 23 & 1749 & $\begin{array}{l}2 \\
6\end{array}$ & $8.86 \%$ \\
\hline OUT\#82 & 57 & 49 & $\begin{array}{c}1.1 \\
7\end{array}$ & 0.05681 & $\begin{array}{c}0.006 \\
71\end{array}$ & $\begin{array}{c}0.7537 \\
9\end{array}$ & $\begin{array}{c}0.085 \\
61\end{array}$ & $\begin{array}{c}0.0964 \\
0\end{array}$ & $\begin{array}{c}0.003 \\
80\end{array}$ & 484 & & & 50 & 593 & $\begin{array}{l}2 \\
2\end{array}$ & $-3.88 \%$ \\
\hline OUT\#83 & 440 & 167 & $\begin{array}{c}2.6 \\
4\end{array}$ & 0.06501 & $\begin{array}{c}0.003 \\
58\end{array}$ & $\begin{array}{c}0.9676 \\
4\end{array}$ & $\begin{array}{c}0.050 \\
71\end{array}$ & $\begin{array}{c}0.1081 \\
4\end{array}$ & $\begin{array}{c}0.002 \\
37\end{array}$ & & & 687 & 26 & 662 & $\begin{array}{l}1 \\
4\end{array}$ & $3.78 \%$ \\
\hline OUT\#84 & 60 & 74 & $\begin{array}{c}0.8 \\
2\end{array}$ & 0.10029 & $\begin{array}{c}0.004 \\
23\end{array}$ & $\begin{array}{c}3.9073 \\
7\end{array}$ & $\begin{array}{c}0.157 \\
16\end{array}$ & $\begin{array}{c}0.2830 \\
7\end{array}$ & $\begin{array}{c}0.006 \\
39\end{array}$ & 1629 & 42 & 1615 & 33 & 1607 & $\begin{array}{l}3 \\
2\end{array}$ & $1.37 \%$ \\
\hline OUT\#85 & 309 & 702 & $\begin{array}{c}0.4 \\
4\end{array}$ & 0.05901 & $\begin{array}{c}0.003 \\
92\end{array}$ & $\begin{array}{c}0.6499 \\
2\end{array}$ & $\begin{array}{c}0.041 \\
60\end{array}$ & $\begin{array}{c}0.0798 \\
8\end{array}$ & $\begin{array}{c}0.001 \\
41\end{array}$ & 568 & $\begin{array}{c}14 \\
9\end{array}$ & 508 & 26 & 495 & 8 & $2.63 \%$ \\
\hline OUT\#86 & 104 & 84 & $\begin{array}{c}1.2 \\
4\end{array}$ & 0.06123 & $\begin{array}{c}0.004 \\
95\end{array}$ & $\begin{array}{c}0.8384 \\
4\end{array}$ & $\begin{array}{c}0.064 \\
68\end{array}$ & $\begin{array}{c}0.0994 \\
9\end{array}$ & $\begin{array}{c}0.002 \\
95\end{array}$ & 647 & $\begin{array}{c}11 \\
5\end{array}$ & 618 & 36 & 611 & $\begin{array}{l}1 \\
7\end{array}$ & $1.15 \%$ \\
\hline OUT\#87 & 22 & 39 & $\begin{array}{c}0.5 \\
7\end{array}$ & 0.04605 & $\begin{array}{c}0.025 \\
60\end{array}$ & $\begin{array}{c}0.3119 \\
4\end{array}$ & $\begin{array}{c}0.171 \\
01\end{array}$ & $\begin{array}{c}0.0491 \\
3\end{array}$ & $\begin{array}{c}0.004 \\
56\end{array}$ & & $\begin{array}{c}93 \\
1\end{array}$ & 276 & $\begin{array}{c}13 \\
2\end{array}$ & 309 & $\begin{array}{l}2 \\
8\end{array}$ & $-10.68 \%$ \\
\hline OUT\#88 & 128 & 252 & $\begin{array}{c}0.5 \\
1\end{array}$ & 0.07066 & $\begin{array}{c}0.003 \\
32\end{array}$ & $\begin{array}{c}1.0352 \\
7\end{array}$ & $\begin{array}{c}0.046 \\
10\end{array}$ & $\begin{array}{c}0.1064 \\
6\end{array}$ & $\begin{array}{c}0.002 \\
12\end{array}$ & 948 & 59 & 722 & 23 & 652 & $\begin{array}{l}1 \\
2\end{array}$ & $10.74 \%$ \\
\hline OUT\#89 & 124 & 190 & $\begin{array}{c}0.6 \\
5\end{array}$ & 0.06086 & $\begin{array}{c}0.003 \\
16\end{array}$ & $\begin{array}{c}0.9465 \\
0\end{array}$ & $\begin{array}{c}0.046 \\
96\end{array}$ & $\begin{array}{c}0.1130 \\
1\end{array}$ & $\begin{array}{c}0.002 \\
31\end{array}$ & 634 & 72 & 676 & 24 & 690 & $\begin{array}{l}1 \\
3\end{array}$ & $-2.03 \%$ \\
\hline OUT\#90 & 84 & 114 & $\begin{array}{c}0.7 \\
4\end{array}$ & 0.07226 & $\begin{array}{c}0.005 \\
65\end{array}$ & $\begin{array}{c}1.1785 \\
8\end{array}$ & $\begin{array}{c}0.087 \\
29\end{array}$ & $\begin{array}{c}0.1185 \\
1\end{array}$ & $\begin{array}{c}0.003 \\
74\end{array}$ & 993 & 99 & 791 & 41 & 722 & $\begin{array}{l}2 \\
2\end{array}$ & $9.56 \%$ \\
\hline OUT\#91 & 151 & 178 & $\begin{array}{c}0.8 \\
5\end{array}$ & 0.07130 & $\begin{array}{c}0.003 \\
26\end{array}$ & $\begin{array}{c}1.2880 \\
8\end{array}$ & $\begin{array}{c}0.055 \\
92\end{array}$ & $\begin{array}{c}0.1312 \\
9\end{array}$ & $\begin{array}{c}0.002 \\
60\end{array}$ & 966 & 56 & 840 & 25 & 795 & $\begin{array}{l}1 \\
5\end{array}$ & $5.66 \%$ \\
\hline OUT\#92 & 59 & 91 & $\begin{array}{c}0.6 \\
5\end{array}$ & 0.06472 & $\begin{array}{c}0.004 \\
82\end{array}$ & $\begin{array}{c}0.8821 \\
7\end{array}$ & $\begin{array}{c}0.062 \\
60\end{array}$ & $\begin{array}{c}0.0990 \\
5\end{array}$ & $\begin{array}{c}0.002 \\
80\end{array}$ & 765 & $\begin{array}{c}10 \\
2\end{array}$ & 642 & 34 & 609 & $\begin{array}{l}1 \\
6\end{array}$ & $5.42 \%$ \\
\hline OUT\#93 & 389 & 409 & $\begin{array}{c}0.9 \\
5\end{array}$ & 0.05971 & $\begin{array}{c}0.005 \\
16\end{array}$ & $\begin{array}{c}0.8067 \\
4\end{array}$ & $\begin{array}{c}0.067 \\
98\end{array}$ & $\begin{array}{c}0.0979 \\
9\end{array}$ & $\begin{array}{c}0.001 \\
88\end{array}$ & 593 & $\begin{array}{c}19 \\
4\end{array}$ & 601 & 38 & 603 & $\begin{array}{l}1 \\
1\end{array}$ & $-0.33 \%$ \\
\hline OUT\#94 & 180 & 238 & $\begin{array}{c}0.7 \\
6\end{array}$ & 0.23136 & $\begin{array}{c}0.006 \\
73\end{array}$ & $\begin{array}{c}13.464 \\
34\end{array}$ & $\begin{array}{c}0.321 \\
45\end{array}$ & $\begin{array}{c}0.4220 \\
8\end{array}$ & $\begin{array}{c}0.007 \\
01\end{array}$ & 3061 & 48 & 2713 & 23 & 2270 & $\begin{array}{l}3 \\
2\end{array}$ & $34.85 \%$ \\
\hline OUT\#95 & 119 & 122 & $\begin{array}{c}0.9 \\
7\end{array}$ & 0.07883 & $\begin{array}{c}0.006 \\
53\end{array}$ & $\begin{array}{c}1.6268 \\
1\end{array}$ & $\begin{array}{c}0.127 \\
94\end{array}$ & $\begin{array}{c}0.1499 \\
8\end{array}$ & $\begin{array}{c}0.005 \\
16\end{array}$ & 1168 & $\begin{array}{c}10 \\
2\end{array}$ & 981 & 49 & 901 & $\begin{array}{l}2 \\
9\end{array}$ & $8.88 \%$ \\
\hline
\end{tabular}




\begin{tabular}{|c|c|c|c|c|c|c|c|c|c|c|c|c|c|c|c|c|}
\hline OUT\#96 & 224 & 344 & $\begin{array}{c}0.6 \\
5\end{array}$ & 0.17391 & $\begin{array}{c}0.004 \\
63\end{array}$ & $\begin{array}{c}8.0572 \\
9\end{array}$ & $\begin{array}{c}0.180 \\
79\end{array}$ & $\begin{array}{c}0.3360 \\
1\end{array}$ & $\begin{array}{c}0.004 \\
83\end{array}$ & 2596 & 45 & 2237 & 20 & 1867 & $\begin{array}{l}2 \\
3\end{array}$ & $39.05 \%$ \\
\hline OUT\#97 & 172 & 192 & $\begin{array}{c}0.9 \\
0\end{array}$ & 0.11606 & $\begin{array}{c}0.002 \\
84\end{array}$ & $\begin{array}{c}5.0573 \\
6\end{array}$ & $\begin{array}{c}0.118 \\
07\end{array}$ & $\begin{array}{c}0.3167 \\
1\end{array}$ & $\begin{array}{c}0.004 \\
95\end{array}$ & 1896 & 21 & 1829 & 20 & 1774 & $\begin{array}{l}2 \\
4\end{array}$ & $6.88 \%$ \\
\hline OUT\#98 & 23 & 676 & $\begin{array}{c}0.0 \\
3\end{array}$ & 0.12719 & $\begin{array}{c}0.002 \\
00\end{array}$ & $\begin{array}{c}6.2915 \\
7\end{array}$ & $\begin{array}{c}0.072 \\
98\end{array}$ & $\begin{array}{c}0.3587 \\
7\end{array}$ & $\begin{array}{c}0.003 \\
82\end{array}$ & 2059 & 28 & 2017 & 10 & 1976 & $\begin{array}{l}1 \\
8\end{array}$ & $4.20 \%$ \\
\hline OUT\#99 & 114 & 535 & $\begin{array}{c}0.2 \\
1\end{array}$ & 0.05839 & $\begin{array}{c}0.001 \\
92\end{array}$ & $\begin{array}{c}0.8046 \\
3\end{array}$ & $\begin{array}{c}0.025 \\
28\end{array}$ & $\begin{array}{c}0.1001 \\
6\end{array}$ & $\begin{array}{c}0.001 \\
45\end{array}$ & 544 & & & 14 & 615 & 8 & $-2.60 \%$ \\
\hline OUT\#100 & 49 & 111 & $\begin{array}{c}0.4 \\
4\end{array}$ & 0.13410 & $\begin{array}{c}0.003 \\
44\end{array}$ & $\begin{array}{c}7.4594 \\
3\end{array}$ & $\begin{array}{c}0.184 \\
47\end{array}$ & $\begin{array}{c}0.4043 \\
2\end{array}$ & $\begin{array}{c}0.007 \\
03\end{array}$ & & & 21 & 22 & 2189 & $\begin{array}{l}3 \\
2\end{array}$ & $-1.69 \%$ \\
\hline \multicolumn{17}{|l|}{ MB2 } \\
\hline MB2\#01 & 249 & 346 & $\begin{array}{c}0.7 \\
2\end{array}$ & 0.07367 & $\begin{array}{c}0.007 \\
52\end{array}$ & $\begin{array}{c}0.8162 \\
7\end{array}$ & $\begin{array}{c}0.080 \\
76\end{array}$ & $\begin{array}{c}0.0803 \\
6\end{array}$ & $\begin{array}{r}0.00 \\
01\end{array}$ & 1032 & $\begin{array}{c}21 \\
5\end{array}$ & 606 & 45 & 498 & $\begin{array}{l}1 \\
2\end{array}$ & $21.69 \%$ \\
\hline MB2\#02 & 38 & 33 & $\begin{array}{c}1.1 \\
5\end{array}$ & 0.12039 & $\begin{array}{c}0.007 \\
49\end{array}$ & $\begin{array}{c}5.4559 \\
7\end{array}$ & $\begin{array}{c}0.323 \\
81\end{array}$ & $\begin{array}{c}0.3288 \\
1\end{array}$ & $\begin{array}{c}0.011 \\
43\end{array}$ & 1962 & 58 & 1894 & 51 & 1833 & $\begin{array}{l}5 \\
5\end{array}$ & $7.04 \%$ \\
\hline MB2\#03 & 167 & 160 & $\begin{array}{l}1.0 \\
5\end{array}$ & 0.06003 & $\begin{array}{c}0.003 \\
65\end{array}$ & $\begin{array}{c}0.7844 \\
2\end{array}$ & $\begin{array}{c}0.045 \\
57\end{array}$ & $\begin{array}{c}0.0948 \\
2\end{array}$ & $\begin{array}{c}0.002 \\
16\end{array}$ & 605 & 86 & 588 & 26 & 584 & $\begin{array}{l}1 \\
3\end{array}$ & $0.68 \%$ \\
\hline MB2\#04 & 116 & 229 & $\begin{array}{c}0.5 \\
1\end{array}$ & 0.11447 & $\begin{array}{c}0.002 \\
41\end{array}$ & $\begin{array}{c}5.2473 \\
6\end{array}$ & $\begin{array}{c}0.105 \\
67\end{array}$ & $\begin{array}{c}0.3326 \\
2\end{array}$ & $\begin{array}{c}0.004 \\
77\end{array}$ & 1872 & 18 & 1860 & 17 & 1851 & $\begin{array}{l}2 \\
3\end{array}$ & $1.13 \%$ \\
\hline MB2\#05 & 437 & 616 & $\begin{array}{c}0.7 \\
1\end{array}$ & 0.05932 & $\begin{array}{c}0.003 \\
83\end{array}$ & $\begin{array}{c}0.7384 \\
0\end{array}$ & $\begin{array}{c}0.046 \\
23\end{array}$ & $\begin{array}{c}0.0902 \\
7\end{array}$ & $\begin{array}{c}0.001 \\
44\end{array}$ & 579 & $\begin{array}{c}14 \\
5\end{array}$ & 561 & 27 & 557 & 9 & $0.72 \%$ \\
\hline MB2\#06 & 59 & 59 & $\begin{array}{c}0.9 \\
9\end{array}$ & & $\begin{array}{c}0.009 \\
24\end{array}$ & $\begin{array}{c}3.7846 \\
5\end{array}$ & $\begin{array}{c}0.298 \\
02\end{array}$ & $\begin{array}{c}0.2480 \\
3\end{array}$ & $\begin{array}{c}0.006 \\
89\end{array}$ & 1810 & $\begin{array}{c}15 \\
7\end{array}$ & 1589 & 63 & 1428 & $\begin{array}{l}3 \\
6\end{array}$ & $26.75 \%$ \\
\hline MB2\#07 & 33 & 37 & $\begin{array}{c}0.8 \\
9\end{array}$ & 0.06717 & $\begin{array}{c}0.008 \\
24\end{array}$ & $\begin{array}{c}0.9757 \\
5\end{array}$ & $\begin{array}{c}0.113 \\
87\end{array}$ & $\begin{array}{c}0.1053 \\
9\end{array}$ & $\begin{array}{c}0.004 \\
82\end{array}$ & 843 & $\begin{array}{c}16 \\
8\end{array}$ & 691 & 59 & 646 & $\begin{array}{l}2 \\
8\end{array}$ & $6.97 \%$ \\
\hline MB2\#08 & 169 & 122 & $\begin{array}{c}1.3 \\
8\end{array}$ & 0.12259 & $\begin{array}{c}0.003 \\
20\end{array}$ & $\begin{array}{c}6.2336 \\
7\end{array}$ & $\begin{array}{c}0.155 \\
88\end{array}$ & $\begin{array}{c}0.3689 \\
5\end{array}$ & $\begin{array}{c}0.006 \\
30\end{array}$ & 1994 & 22 & 2009 & 22 & 2024 & $\begin{array}{l}3 \\
0\end{array}$ & $-1.48 \%$ \\
\hline MB2\#09 & 51 & 47 & $\begin{array}{c}1.0 \\
9\end{array}$ & 0564 & $\begin{array}{c}0.005 \\
13\end{array}$ & $\begin{array}{c}4.5206 \\
2\end{array}$ & $\begin{array}{c}0.209 \\
62\end{array}$ & $\begin{array}{c}0.3104 \\
9\end{array}$ & $\begin{array}{c}0.008 \\
14\end{array}$ & 1725 & 48 & 1735 & 39 & 1743 & $\begin{array}{l}4 \\
0\end{array}$ & $-1.03 \%$ \\
\hline MB2\#10 & 43 & 64 & $\begin{array}{c}0.6 \\
7\end{array}$ & 0.06414 & $\begin{array}{c}0.006 \\
54\end{array}$ & $\begin{array}{c}0.8002 \\
8\end{array}$ & $\begin{array}{c}0.078 \\
05\end{array}$ & $\begin{array}{c}0.0905 \\
3\end{array}$ & $\begin{array}{c}0.003 \\
23\end{array}$ & 746 & $\begin{array}{c}14 \\
6\end{array}$ & 597 & 44 & 559 & $\begin{array}{l}1 \\
9\end{array}$ & $6.80 \%$ \\
\hline$M B 2 \# 11$ & 46 & 64 & $\begin{array}{c}0.7 \\
2\end{array}$ & 0.13943 & $\begin{array}{c}0.008 \\
47\end{array}$ & $\begin{array}{c}6.6314 \\
7\end{array}$ & $\begin{array}{c}0.383 \\
13\end{array}$ & $\begin{array}{c}0.3450 \\
8\end{array}$ & $\begin{array}{c}0.012 \\
50\end{array}$ & 2220 & 53 & 2064 & 51 & 1911 & $\begin{array}{l}6 \\
0\end{array}$ & $16.17 \%$ \\
\hline MB2\#12 & 206 & 349 & $\begin{array}{c}0.5 \\
9\end{array}$ & 0.06191 & $\begin{array}{c}0.002 \\
53\end{array}$ & $\begin{array}{c}0.7972 \\
4\end{array}$ & $\begin{array}{c}0.031 \\
06\end{array}$ & $\begin{array}{c}0.0934 \\
3\end{array}$ & $\begin{array}{c}0.001 \\
60\end{array}$ & 671 & 54 & 595 & 18 & 576 & 9 & $3.30 \%$ \\
\hline
\end{tabular}




\begin{tabular}{|c|c|c|c|c|c|c|c|c|c|c|c|c|c|c|c|c|}
\hline MB2\#13 & 91 & 92 & $\begin{array}{c}1.0 \\
0\end{array}$ & 0.06799 & $\begin{array}{c}0.005 \\
57\end{array}$ & $\begin{array}{c}1.0002 \\
5\end{array}$ & $\begin{array}{l}0.078 \\
02\end{array}$ & $\begin{array}{c}0.1067 \\
4\end{array}$ & $\begin{array}{c}0.003 \\
34\end{array}$ & 868 & $\begin{array}{c}11 \\
0\end{array}$ & 704 & 40 & 654 & $\begin{array}{l}1 \\
9\end{array}$ & $7.65 \%$ \\
\hline MB2\#14 & 123 & 303 & $\begin{array}{c}0.4 \\
1\end{array}$ & 0.05604 & $\begin{array}{c}0.002 \\
81\end{array}$ & $\begin{array}{c}0.6047 \\
8\end{array}$ & $\begin{array}{c}0.029 \\
02\end{array}$ & $\begin{array}{c}0.0783 \\
0\end{array}$ & $\begin{array}{c}0.001 \\
48\end{array}$ & 454 & 73 & 480 & 18 & 486 & 9 & $-1.23 \%$ \\
\hline$M B 2 \# 15$ & 129 & 123 & $\begin{array}{c}1.0 \\
5\end{array}$ & 0.12108 & $\begin{array}{c}0.004 \\
08\end{array}$ & $\begin{array}{c}4.7407 \\
1\end{array}$ & $\begin{array}{c}0.151 \\
21\end{array}$ & $\begin{array}{c}0.2840 \\
9\end{array}$ & $\begin{array}{c}0.005 \\
68\end{array}$ & 1972 & 30 & 1774 & 27 & 1612 & 2 & $22.33 \%$ \\
\hline MB2\#16 & 65 & 85 & $\begin{array}{c}0.7 \\
7\end{array}$ & 0.14039 & $\begin{array}{c}0.004 \\
18\end{array}$ & $\begin{array}{c}7.8450 \\
3\end{array}$ & $\begin{array}{c}0.224 \\
53\end{array}$ & $\begin{array}{c}0.4054 \\
3\end{array}$ & $\begin{array}{c}0.008 \\
08\end{array}$ & 2232 & & 213 & 26 & 2194 & $\begin{array}{l}3 \\
7\end{array}$ & $1.73 \%$ \\
\hline MB2\#17 & 73 & 147 & $\begin{array}{c}0.4 \\
9\end{array}$ & 0.07314 & $\begin{array}{c}0.003 \\
61\end{array}$ & $\begin{array}{c}1.3640 \\
2\end{array}$ & $\begin{array}{c}0.063 \\
97\end{array}$ & $\begin{array}{c}0.1353 \\
1\end{array}$ & $\begin{array}{c}0.002 \\
89\end{array}$ & 1018 & & 874 & 27 & 818 & $\begin{array}{l}1 \\
6\end{array}$ & $6.85 \%$ \\
\hline MB2\#18 & 96 & 133 & $\begin{array}{c}0.7 \\
2\end{array}$ & 0.06148 & $\begin{array}{c}0.003 \\
89\end{array}$ & $\begin{array}{c}0.8837 \\
3\end{array}$ & $\begin{array}{c}0.053 \\
40\end{array}$ & $\begin{array}{c}0.1042 \\
9\end{array}$ & $\begin{array}{c}0.002 \\
52\end{array}$ & 65 & 88 & 643 & 29 & 640 & $\begin{array}{l}1 \\
5\end{array}$ & $0.47 \%$ \\
\hline MB2\#19 & 109 & 228 & $\begin{array}{c}0.4 \\
8\end{array}$ & 0.12924 & $\begin{array}{c}0.002 \\
37\end{array}$ & $\begin{array}{c}6.6341 \\
5\end{array}$ & $\begin{array}{c}0.116 \\
19\end{array}$ & $\begin{array}{c}0.3724 \\
5\end{array}$ & $\begin{array}{c}0.005 \\
09\end{array}$ & 2088 & 14 & 2064 & 15 & 2041 & $\begin{array}{l}2 \\
4\end{array}$ & $2.30 \%$ \\
\hline MB2\#20 & 141 & 123 & $\begin{array}{c}1.1 \\
5\end{array}$ & 0.06312 & $\begin{array}{c}0.003 \\
78\end{array}$ & $\begin{array}{c}1.0506 \\
7\end{array}$ & $\begin{array}{c}0.060 \\
06\end{array}$ & $\begin{array}{c}0.1207 \\
8\end{array}$ & $\begin{array}{c}0.002 \\
83\end{array}$ & 712 & 82 & 729 & 30 & 735 & $\begin{array}{l}1 \\
6\end{array}$ & $-0.82 \%$ \\
\hline MB2\#21 & 77 & 138 & $\begin{array}{c}0.5 \\
6\end{array}$ & 0.05700 & $\begin{array}{c}0.003 \\
62\end{array}$ & $\begin{array}{c}0.7590 \\
2\end{array}$ & $\begin{array}{c}0.046 \\
13\end{array}$ & $\begin{array}{c}0.0966 \\
2\end{array}$ & $\begin{array}{c}0.002 \\
27\end{array}$ & 492 & 93 & 573 & 27 & 595 & $\begin{array}{l}1 \\
3\end{array}$ & $-3.70 \%$ \\
\hline$M B 2 \# 22$ & 17 & 18 & $\begin{array}{c}0.9 \\
4\end{array}$ & 0.05080 & $\begin{array}{c}0.010 \\
38\end{array}$ & $\begin{array}{c}0.6734 \\
5\end{array}$ & $\begin{array}{c}0.133 \\
39\end{array}$ & $\begin{array}{c}0.0961 \\
8\end{array}$ & $\begin{array}{c}0.005 \\
67\end{array}$ & 232 & $\begin{array}{c}30 \\
3\end{array}$ & 523 & 81 & 592 & $\begin{array}{l}3 \\
3\end{array}$ & $-11.66 \%$ \\
\hline$M B 2 \# 23$ & 75 & 107 & $\begin{array}{c}0.7 \\
0\end{array}$ & 0.07277 & $\begin{array}{c}0.004 \\
81\end{array}$ & $\begin{array}{c}1.0384 \\
1\end{array}$ & $\begin{array}{c}0.064 \\
97\end{array}$ & $\begin{array}{c}0.1035 \\
3\end{array}$ & $\begin{array}{c}0.002 \\
79\end{array}$ & 1008 & 83 & 723 & 32 & 635 & $\begin{array}{l}1 \\
6\end{array}$ & $13.86 \%$ \\
\hline MB2\#24 & 190 & 150 & $\begin{array}{c}1.2 \\
6\end{array}$ & 0.06770 & $\begin{array}{c}0.003 \\
51\end{array}$ & $\begin{array}{c}0.9801 \\
4\end{array}$ & $\begin{array}{c}0.048 \\
28\end{array}$ & $\begin{array}{c}0.1050 \\
4\end{array}$ & $\begin{array}{c}0.002 \\
21\end{array}$ & 859 & 67 & 694 & 25 & 644 & $\begin{array}{l}1 \\
3\end{array}$ & $7.76 \%$ \\
\hline MB2\#25 & 88 & 85 & $\begin{array}{c}1.0 \\
3\end{array}$ & 0.12180 & $\begin{array}{c}0.004 \\
08\end{array}$ & $\begin{array}{c}5.8260 \\
5\end{array}$ & $\begin{array}{c}0.186 \\
32\end{array}$ & $\begin{array}{c}0.3470 \\
4\end{array}$ & $\begin{array}{c}0.007 \\
10\end{array}$ & 1983 & 30 & 1950 & 28 & 1920 & $\begin{array}{l}3 \\
4\end{array}$ & $3.28 \%$ \\
\hline MB2\#26 & 166 & 113 & $\begin{array}{c}1.4 \\
7\end{array}$ & 0.05884 & $\begin{array}{c}0.003 \\
73\end{array}$ & $\begin{array}{c}0.8435 \\
3\end{array}$ & $\begin{array}{c}0.051 \\
15\end{array}$ & $\begin{array}{c}0.1040 \\
2\end{array}$ & $\begin{array}{c}0.002 \\
48\end{array}$ & 561 & 91 & 621 & 28 & 638 & $\begin{array}{l}1 \\
4\end{array}$ & $-2.66 \%$ \\
\hline MB2\#27 & 85 & 130 & $\begin{array}{c}0.6 \\
5\end{array}$ & 0.05987 & $\begin{array}{c}0.003 \\
95\end{array}$ & $\begin{array}{c}0.8559 \\
6\end{array}$ & $\begin{array}{c}0.054 \\
05\end{array}$ & $\begin{array}{c}0.1037 \\
3\end{array}$ & $\begin{array}{c}0.002 \\
52\end{array}$ & 599 & 95 & 628 & 30 & 636 & $\begin{array}{l}1 \\
5\end{array}$ & $-1.26 \%$ \\
\hline MB2\#28 & 30 & 57 & $\begin{array}{c}0.5 \\
3\end{array}$ & 0.08668 & $\begin{array}{c}0.009 \\
03\end{array}$ & $\begin{array}{c}2.7108 \\
1\end{array}$ & $\begin{array}{c}0.270 \\
91\end{array}$ & $\begin{array}{c}0.2268 \\
3\end{array}$ & $\begin{array}{c}0.006 \\
64\end{array}$ & 1353 & $\begin{array}{c}20 \\
9\end{array}$ & 1331 & 74 & 1318 & $\begin{array}{l}3 \\
5\end{array}$ & $2.66 \%$ \\
\hline MB2\#29 & 85 & 88 & $\begin{array}{c}0.9 \\
7\end{array}$ & 0.12787 & $\begin{array}{c}0.003 \\
77\end{array}$ & $\begin{array}{c}6.6644 \\
5\end{array}$ & $\begin{array}{c}0.188 \\
29\end{array}$ & $\begin{array}{c}0.3781 \\
6\end{array}$ & $\begin{array}{c}0.007 \\
18\end{array}$ & 2069 & 25 & 2068 & 25 & 2068 & $\begin{array}{l}3 \\
4\end{array}$ & $0.05 \%$ \\
\hline MB2\#30 & 82 & 79 & 1.0 & 0.11959 & 0.004 & 5.0671 & 0.164 & 0.3074 & 0.006 & 1950 & 31 & 1831 & 28 & 1728 & 3 & $12.85 \%$ \\
\hline
\end{tabular}




\begin{tabular}{|c|c|c|c|c|c|c|c|c|c|c|c|c|c|c|c|c|}
\hline & & & 4 & & 09 & 4 & 75 & 3 & 23 & & & & & & 1 & \\
\hline MB2\#31 & 96 & 142 & $\begin{array}{c}0.6 \\
7\end{array}$ & 0.12026 & $\begin{array}{c}0.002 \\
83\end{array}$ & $\begin{array}{c}5.4266 \\
4\end{array}$ & $\begin{array}{c}0.121 \\
80\end{array}$ & $\begin{array}{c}0.3273 \\
9\end{array}$ & $\begin{array}{c}0.005 \\
07\end{array}$ & 1960 & 20 & 1889 & 19 & 1826 & $\begin{array}{l}2 \\
5\end{array}$ & $7.34 \%$ \\
\hline MB2\#32 & 307 & 279 & $\begin{array}{c}1.1 \\
0\end{array}$ & 0.07030 & $\begin{array}{c}0.003 \\
24\end{array}$ & $\begin{array}{c}0.8970 \\
8\end{array}$ & $\begin{array}{c}0.039 \\
15\end{array}$ & $\begin{array}{c}0.0925 \\
8\end{array}$ & $\begin{array}{c}0.001 \\
81\end{array}$ & 937 & 58 & 650 & 21 & 571 & $\begin{array}{l}1 \\
1\end{array}$ & $13.84 \%$ \\
\hline MB2\#33 & 174 & 465 & $\begin{array}{c}0.3 \\
7\end{array}$ & 0.06133 & $\begin{array}{c}0.002 \\
02\end{array}$ & $\begin{array}{c}0.8855 \\
8\end{array}$ & $\begin{array}{c}0.027 \\
84\end{array}$ & $\begin{array}{c}0.1047 \\
6\end{array}$ & $\begin{array}{c}0.001 \\
55\end{array}$ & 651 & 42 & & 15 & 642 & 9 & $0.31 \%$ \\
\hline MB2\#34 & 98 & 184 & $\begin{array}{c}0.5 \\
3\end{array}$ & 0.11634 & $\begin{array}{c}0.004 \\
16\end{array}$ & $\begin{array}{c}4.0938 \\
5\end{array}$ & $\begin{array}{c}0.138 \\
09\end{array}$ & $\begin{array}{c}0.2553 \\
0\end{array}$ & $\begin{array}{c}0.005 \\
22\end{array}$ & 100 & & & 28 & 1466 & $\begin{array}{l}2 \\
7\end{array}$ & $29.67 \%$ \\
\hline MB2\#35 & 87 & 161 & $\begin{array}{c}0.5 \\
4\end{array}$ & 0.11733 & $\begin{array}{c}0.002 \\
75\end{array}$ & $\begin{array}{c}5.6657 \\
8\end{array}$ & $\begin{array}{c}0.127 \\
06\end{array}$ & $\begin{array}{c}0.3503 \\
6\end{array}$ & $\begin{array}{c}0.005 \\
43\end{array}$ & 1916 & 20 & 1926 & 19 & 1936 & $\begin{array}{l}2 \\
6\end{array}$ & $-1.03 \%$ \\
\hline MB2\#36 & 79 & 83 & $\begin{array}{c}0.9 \\
5\end{array}$ & 0.06671 & $\begin{array}{c}0.004 \\
68\end{array}$ & $\begin{array}{c}1.0859 \\
3\end{array}$ & $\begin{array}{c}0.072 \\
91\end{array}$ & $\begin{array}{c}0.1181 \\
0\end{array}$ & $\begin{array}{c}0.003 \\
10\end{array}$ & 829 & 96 & 747 & 35 & 720 & $\begin{array}{l}1 \\
8\end{array}$ & $3.75 \%$ \\
\hline MB2\#37 & 112 & 135 & $\begin{array}{c}0.8 \\
3\end{array}$ & 0.06219 & $\begin{array}{c}0.004 \\
14\end{array}$ & $\begin{array}{c}0.9441 \\
4\end{array}$ & $\begin{array}{c}0.060 \\
12\end{array}$ & $\begin{array}{c}0.1101 \\
5\end{array}$ & $\begin{array}{c}0.002 \\
76\end{array}$ & 681 & 93 & 675 & 31 & 674 & $\begin{array}{l}1 \\
6\end{array}$ & $0.15 \%$ \\
\hline$M B 2 \# 38$ & 417 & 551 & $\begin{array}{c}0.7 \\
6\end{array}$ & 0.06887 & $\begin{array}{c}0.004 \\
03\end{array}$ & $\begin{array}{c}0.8306 \\
7\end{array}$ & $\begin{array}{c}0.046 \\
88\end{array}$ & $\begin{array}{c}0.0874 \\
8\end{array}$ & $\begin{array}{c}0.001 \\
37\end{array}$ & 895 & $\begin{array}{c}12 \\
4\end{array}$ & 614 & 26 & 541 & 8 & $13.49 \%$ \\
\hline MB2\#39 & 56 & 91 & $\begin{array}{c}0.6 \\
1\end{array}$ & 0.11348 & $\begin{array}{c}0.003 \\
80\end{array}$ & $\begin{array}{c}4.8371 \\
5\end{array}$ & $\begin{array}{c}0.154 \\
31\end{array}$ & $\begin{array}{c}0.3092 \\
7\end{array}$ & $\begin{array}{c}0.006 \\
09\end{array}$ & 1856 & 31 & 1791 & 27 & 1737 & $\begin{array}{l}3 \\
0\end{array}$ & $6.85 \%$ \\
\hline MB2\#40 & 206 & 178 & $\begin{array}{c}1.1 \\
6\end{array}$ & 0.13953 & $\begin{array}{c}0.006 \\
70\end{array}$ & $\begin{array}{c}7.5547 \\
2\end{array}$ & $\begin{array}{c}0.332 \\
59\end{array}$ & $\begin{array}{c}0.3926 \\
9\end{array}$ & $\begin{array}{c}0.007 \\
50\end{array}$ & 2221 & 85 & 2180 & 39 & 2135 & $\begin{array}{l}3 \\
5\end{array}$ & $4.03 \%$ \\
\hline MB2\#41 & 67 & 80 & $\begin{array}{c}0.8 \\
4\end{array}$ & 0.05733 & $\begin{array}{c}0.004 \\
50\end{array}$ & $\begin{array}{c}0.9913 \\
4\end{array}$ & $\begin{array}{c}0.074 \\
77\end{array}$ & $\begin{array}{c}0.1254 \\
6\end{array}$ & $\begin{array}{c}0.003 \\
55\end{array}$ & 504 & $\begin{array}{c}11 \\
6\end{array}$ & 699 & 38 & 762 & $\begin{array}{l}2 \\
0\end{array}$ & $-8.27 \%$ \\
\hline$M B 2 \# 42$ & 68 & 44 & $\begin{array}{c}1.5 \\
5\end{array}$ & 0.04605 & $\begin{array}{c}0.011 \\
77\end{array}$ & $\begin{array}{c}0.4187 \\
7\end{array}$ & $\begin{array}{c}0.103 \\
53\end{array}$ & $\begin{array}{c}0.0659 \\
6\end{array}$ & $\begin{array}{c}0.004 \\
29\end{array}$ & & $\begin{array}{c}41 \\
7\end{array}$ & 355 & 74 & 412 & $\begin{array}{l}2 \\
6\end{array}$ & $-13.83 \%$ \\
\hline MB2\#43 & 151 & 59 & $\begin{array}{c}2.5 \\
6\end{array}$ & 0.05661 & $\begin{array}{c}0.006 \\
07\end{array}$ & $\begin{array}{c}0.7728 \\
9\end{array}$ & $\begin{array}{c}0.079 \\
42\end{array}$ & $\begin{array}{c}0.0990 \\
6\end{array}$ & $\begin{array}{c}0.003 \\
66\end{array}$ & 476 & $\begin{array}{c}16 \\
3\end{array}$ & 581 & 45 & 609 & $\begin{array}{l}2 \\
1\end{array}$ & $-4.60 \%$ \\
\hline MB2\#44 & 85 & 97 & $\begin{array}{c}0.8 \\
8\end{array}$ & 0.11556 & $\begin{array}{c}0.006 \\
46\end{array}$ & $\begin{array}{c}5.1223 \\
0\end{array}$ & $\begin{array}{c}0.265 \\
81\end{array}$ & $\begin{array}{c}0.3214 \\
8\end{array}$ & $\begin{array}{c}0.006 \\
71\end{array}$ & 1889 & $\begin{array}{c}10 \\
3\end{array}$ & 1840 & 44 & 1797 & $\begin{array}{l}3 \\
3\end{array}$ & $5.12 \%$ \\
\hline$M B 2 \# 45$ & 34 & 27 & $\begin{array}{c}1.2 \\
5\end{array}$ & 0.08968 & $\begin{array}{c}0.012 \\
22\end{array}$ & $\begin{array}{c}1.1131 \\
6\end{array}$ & $\begin{array}{c}0.142 \\
31\end{array}$ & $\begin{array}{c}0.0900 \\
6\end{array}$ & $\begin{array}{c}0.004 \\
98\end{array}$ & 1419 & $\begin{array}{c}16 \\
1\end{array}$ & 760 & 68 & 556 & $\begin{array}{l}2 \\
9\end{array}$ & $36.69 \%$ \\
\hline MB2\#46 & 67 & 69 & $\begin{array}{c}0.9 \\
8\end{array}$ & 0.12392 & $\begin{array}{c}0.004 \\
16\end{array}$ & $\begin{array}{c}6.5759 \\
7\end{array}$ & $\begin{array}{c}0.212 \\
44\end{array}$ & $\begin{array}{c}0.3850 \\
0\end{array}$ & $\begin{array}{c}0.008 \\
06\end{array}$ & 2013 & 30 & 2056 & 28 & 2100 & $\begin{array}{l}3 \\
8\end{array}$ & $-4.14 \%$ \\
\hline MB2\#47 & 90 & 102 & $\begin{array}{c}0.8 \\
8\end{array}$ & 0.10096 & $\begin{array}{c}0.006 \\
44\end{array}$ & $\begin{array}{c}4.0196 \\
0\end{array}$ & $\begin{array}{c}0.241 \\
30\end{array}$ & $\begin{array}{c}0.2887 \\
6\end{array}$ & $\begin{array}{c}0.006 \\
23\end{array}$ & 1642 & $\begin{array}{c}12 \\
2\end{array}$ & 1638 & 49 & 1635 & $\begin{array}{l}3 \\
1\end{array}$ & $0.43 \%$ \\
\hline
\end{tabular}




\begin{tabular}{|c|c|c|c|c|c|c|c|c|c|c|c|c|c|c|c|c|}
\hline MB2\#48 & 139 & 341 & $\begin{array}{c}0.4 \\
1\end{array}$ & 0.06158 & $\begin{array}{c}0.002 \\
83\end{array}$ & $\begin{array}{c}0.8098 \\
2\end{array}$ & $\begin{array}{c}0.035 \\
48\end{array}$ & $\begin{array}{c}0.0954 \\
1\end{array}$ & $\begin{array}{c}0.001 \\
78\end{array}$ & 660 & 62 & 602 & 20 & 587 & $\begin{array}{l}1 \\
0\end{array}$ & $2.56 \%$ \\
\hline MB2\#49 & 133 & 150 & $\begin{array}{c}0.8 \\
9\end{array}$ & 0.06271 & $\begin{array}{c}0.003 \\
63\end{array}$ & $\begin{array}{c}0.9539 \\
0\end{array}$ & $\begin{array}{c}0.052 \\
76\end{array}$ & $\begin{array}{c}0.1103 \\
6\end{array}$ & $\begin{array}{c}0.002 \\
47\end{array}$ & 698 & 80 & 680 & 27 & 675 & $\begin{array}{l}1 \\
4\end{array}$ & $0.74 \%$ \\
\hline MB2\#50 & 41 & 93 & $\begin{array}{c}0.4 \\
4\end{array}$ & 0.06645 & $\begin{array}{c}0.004 \\
43\end{array}$ & $\begin{array}{c}1.2461 \\
6\end{array}$ & $\begin{array}{c}0.079 \\
57\end{array}$ & $\begin{array}{c}0.1360 \\
7\end{array}$ & $\begin{array}{c}0.003 \\
52\end{array}$ & 821 & 90 & 822 & 36 & 822 & $\begin{array}{l}2 \\
0\end{array}$ & $0.00 \%$ \\
\hline MB2\#51 & 177 & 120 & $\begin{array}{c}1.4 \\
7\end{array}$ & 0.06495 & $\begin{array}{c}0.004 \\
13\end{array}$ & $\begin{array}{c}0.8610 \\
0\end{array}$ & $\begin{array}{c}0.052 \\
14\end{array}$ & $\begin{array}{c}0.0961 \\
8\end{array}$ & $\begin{array}{c}0.002 \\
35\end{array}$ & 773 & & 631 & 28 & 592 & $\begin{array}{l}1 \\
4\end{array}$ & $6.59 \%$ \\
\hline MB2\#52 & 64 & 105 & $\begin{array}{c}0.6 \\
1\end{array}$ & 0.10982 & $\begin{array}{c}0.003 \\
44\end{array}$ & $\begin{array}{c}4.6165 \\
0\end{array}$ & $\begin{array}{c}0.137 \\
90\end{array}$ & $\begin{array}{c}0.3049 \\
9\end{array}$ & $\begin{array}{c}0.005 \\
63\end{array}$ & 179 & & 17 & 25 & 1716 & $\begin{array}{l}2 \\
8\end{array}$ & $4.66 \%$ \\
\hline MB2\#53 & 57 & 110 & $\begin{array}{c}0.5 \\
2\end{array}$ & 0.17957 & $\begin{array}{c}0.003 \\
55\end{array}$ & $\begin{array}{c}12.797 \\
26\end{array}$ & $\begin{array}{c}0.246 \\
82\end{array}$ & $\begin{array}{c}0.5170 \\
4\end{array}$ & $\begin{array}{c}0.008 \\
30\end{array}$ & 2649 & 14 & 2665 & 18 & 2687 & $\begin{array}{l}3 \\
5\end{array}$ & $-1.41 \%$ \\
\hline MB2\#54 & 106 & 160 & $\begin{array}{c}0.6 \\
6\end{array}$ & 0.06394 & $\begin{array}{c}0.003 \\
34\end{array}$ & $\begin{array}{c}0.9463 \\
5\end{array}$ & $\begin{array}{c}0.047 \\
03\end{array}$ & $\begin{array}{c}0.1073 \\
8\end{array}$ & $\begin{array}{c}0.002 \\
25\end{array}$ & 740 & 70 & 676 & 25 & 658 & $\begin{array}{l}1 \\
3\end{array}$ & $2.74 \%$ \\
\hline MB2\#55 & 137 & 348 & $\begin{array}{c}0.3 \\
9\end{array}$ & 0.06205 & $\begin{array}{c}0.003 \\
65\end{array}$ & $\begin{array}{c}0.8147 \\
6\end{array}$ & $\begin{array}{c}0.045 \\
90\end{array}$ & $\begin{array}{c}0.0952 \\
4\end{array}$ & $\begin{array}{c}0.001 \\
62\end{array}$ & 676 & $\begin{array}{c}12 \\
9\end{array}$ & 605 & 26 & 586 & $\begin{array}{l}1 \\
0\end{array}$ & $3.24 \%$ \\
\hline MB2\#56 & 88 & 46 & $\begin{array}{c}1.9 \\
0\end{array}$ & 0.11408 & $\begin{array}{c}0.005 \\
36\end{array}$ & $\begin{array}{c}5.6356 \\
6\end{array}$ & $\begin{array}{c}0.254 \\
45\end{array}$ & $\begin{array}{c}0.3584 \\
1\end{array}$ & $\begin{array}{c}0.009 \\
58\end{array}$ & 1865 & 45 & 1922 & 39 & 1975 & $\begin{array}{l}4 \\
5\end{array}$ & $-5.57 \%$ \\
\hline MB2\#57 & 178 & 181 & $\begin{array}{c}0.9 \\
8\end{array}$ & 0.05870 & $\begin{array}{c}0.006 \\
93\end{array}$ & 0.7240 & $\begin{array}{c}0.083 \\
57\end{array}$ & $\begin{array}{c}0.0894 \\
7\end{array}$ & $\begin{array}{c}0.002 \\
24\end{array}$ & 556 & $\begin{array}{c}26 \\
7\end{array}$ & 553 & 49 & 552 & $\begin{array}{l}1 \\
3\end{array}$ & $0.18 \%$ \\
\hline MB2\#58 & 198 & 279 & $\begin{array}{c}0.7 \\
1\end{array}$ & 0.06161 & $\begin{array}{c}0.002 \\
63\end{array}$ & $\begin{array}{c}0.8994 \\
4\end{array}$ & $\begin{array}{c}0.036 \\
59\end{array}$ & $\begin{array}{c}0.1059 \\
2\end{array}$ & $\begin{array}{c}0.001 \\
87\end{array}$ & 661 & 57 & 651 & 20 & 649 & $\begin{array}{l}1 \\
1\end{array}$ & $0.31 \%$ \\
\hline MB2\#59 & 66 & 97 & $\begin{array}{c}0.6 \\
8\end{array}$ & 0.13866 & $\begin{array}{c}0.003 \\
77\end{array}$ & $\begin{array}{c}7.7291 \\
0\end{array}$ & $\begin{array}{c}0.201 \\
98\end{array}$ & $\begin{array}{c}0.4044 \\
0\end{array}$ & $\begin{array}{c}0.007 \\
43\end{array}$ & 2211 & 22 & 2200 & 23 & 2189 & $\begin{array}{l}3 \\
4\end{array}$ & $1.01 \%$ \\
\hline MB2\#60 & 91 & 55 & $\begin{array}{c}1.6 \\
6\end{array}$ & 0.06180 & $\begin{array}{c}0.006 \\
35\end{array}$ & $\begin{array}{c}0.8813 \\
8\end{array}$ & $\begin{array}{c}0.086 \\
68\end{array}$ & $\begin{array}{c}0.1034 \\
8\end{array}$ & $\begin{array}{c}0.003 \\
76\end{array}$ & 667 & $\begin{array}{l}14 \\
9\end{array}$ & 642 & 47 & 635 & $\begin{array}{l}2 \\
2\end{array}$ & $1.10 \%$ \\
\hline MB2\#61 & 89 & 145 & $\begin{array}{c}0.6 \\
1\end{array}$ & 0.11423 & $\begin{array}{c}0.003 \\
05\end{array}$ & $\begin{array}{c}5.1673 \\
5\end{array}$ & $\begin{array}{c}0.131 \\
84\end{array}$ & $\begin{array}{c}0.3281 \\
9\end{array}$ & $\begin{array}{c}0.005 \\
47\end{array}$ & 1868 & 24 & 1847 & 22 & 1830 & $\begin{array}{l}2 \\
7\end{array}$ & $2.08 \%$ \\
\hline MB2\#62 & 137 & 89 & $\begin{array}{c}1.5 \\
5\end{array}$ & 0.12462 & $\begin{array}{c}0.006 \\
19\end{array}$ & $\begin{array}{c}5.1835 \\
1\end{array}$ & $\begin{array}{c}0.244 \\
26\end{array}$ & $\begin{array}{c}0.3017 \\
7\end{array}$ & $\begin{array}{c}0.008 \\
53\end{array}$ & 2023 & 45 & 1850 & 40 & 1700 & $\begin{array}{l}4 \\
2\end{array}$ & $19.00 \%$ \\
\hline MB2\#63 & 228 & 464 & $\begin{array}{c}0.4 \\
9\end{array}$ & 0.18713 & $\begin{array}{c}0.002 \\
21\end{array}$ & $\begin{array}{c}13.653 \\
75\end{array}$ & $\begin{array}{c}0.156 \\
69\end{array}$ & $\begin{array}{c}0.5293 \\
5\end{array}$ & $\begin{array}{c}0.006 \\
19\end{array}$ & 2717 & 9 & 2726 & 11 & 2739 & $\begin{array}{l}2 \\
6\end{array}$ & $-0.80 \%$ \\
\hline MB2\#64 & 60 & 37 & $\begin{array}{c}1.6 \\
1\end{array}$ & 0.12179 & $\begin{array}{c}0.006 \\
56\end{array}$ & $\begin{array}{c}5.3039 \\
5\end{array}$ & $\begin{array}{c}0.271 \\
70\end{array}$ & $\begin{array}{c}0.3159 \\
6\end{array}$ & $\begin{array}{c}0.009 \\
64\end{array}$ & 1983 & 50 & 1869 & 44 & 1770 & $\begin{array}{l}4 \\
7\end{array}$ & $12.03 \%$ \\
\hline MB2\#65 & 289 & 497 & 0.5 & 0.06227 & 0.002 & 0.8807 & 0.029 & 0.1026 & 0.001 & 683 & 45 & 641 & 16 & 630 & 9 & $1.75 \%$ \\
\hline
\end{tabular}




\begin{tabular}{|c|c|c|c|c|c|c|c|c|c|c|c|c|c|c|c|c|}
\hline & & & 8 & & 17 & 7 & 17 & 1 & 58 & & & & & & & \\
\hline MB2\#66 & 54 & 79 & $\begin{array}{c}0.6 \\
8\end{array}$ & 0.12042 & $\begin{array}{c}0.004 \\
63\end{array}$ & $\begin{array}{c}5.7771 \\
1\end{array}$ & $\begin{array}{c}0.212 \\
28\end{array}$ & $\begin{array}{c}0.3480 \\
5\end{array}$ & $\begin{array}{c}0.007 \\
97\end{array}$ & 1962 & 35 & 1943 & 32 & 1925 & $\begin{array}{l}3 \\
8\end{array}$ & $1.92 \%$ \\
\hline MB2\#67 & 117 & 100 & $\begin{array}{c}1.1 \\
7\end{array}$ & 0.05850 & $\begin{array}{c}0.004 \\
76\end{array}$ & $\begin{array}{c}0.7934 \\
7\end{array}$ & $\begin{array}{c}0.061 \\
78\end{array}$ & $\begin{array}{c}0.0984 \\
0\end{array}$ & $\begin{array}{c}0.002 \\
89\end{array}$ & 549 & $\begin{array}{c}11 \\
9\end{array}$ & 593 & 35 & 605 & $\begin{array}{l}1 \\
7\end{array}$ & $-1.98 \%$ \\
\hline MB2\#68 & 120 & 226 & $\begin{array}{c}0.5 \\
3\end{array}$ & 0.06291 & $\begin{array}{c}0.003 \\
22\end{array}$ & $\begin{array}{c}0.8498 \\
0\end{array}$ & $\begin{array}{c}0.041 \\
41\end{array}$ & $\begin{array}{c}0.0980 \\
0\end{array}$ & $\begin{array}{c}0.001 \\
99\end{array}$ & 705 & 69 & 625 & 23 & 603 & $\begin{array}{l}1 \\
2\end{array}$ & $3.65 \%$ \\
\hline MB2\#69 & 44 & 61 & $\begin{array}{c}0.7 \\
2\end{array}$ & 0.09240 & $\begin{array}{c}0.011 \\
90\end{array}$ & $\begin{array}{c}2.4382 \\
4\end{array}$ & $\begin{array}{c}0.301 \\
77\end{array}$ & $\begin{array}{c}0.1913 \\
8\end{array}$ & $\begin{array}{c}0.006 \\
81\end{array}$ & 47 & & & 89 & 1129 & $\begin{array}{l}3 \\
7\end{array}$ & $30.74 \%$ \\
\hline MB2\#70 & 80 & 116 & $\begin{array}{c}0.6 \\
9\end{array}$ & 0.12111 & $\begin{array}{c}0.003 \\
36\end{array}$ & $\begin{array}{c}5.7408 \\
6\end{array}$ & $\begin{array}{c}0.152 \\
43\end{array}$ & $\begin{array}{c}0.3439 \\
1\end{array}$ & $\begin{array}{c}0.006 \\
03\end{array}$ & 07 & 24 & 1938 & 23 & 1905 & $\begin{array}{l}2 \\
9\end{array}$ & $3.57 \%$ \\
\hline MB2\#71 & 161 & 199 & $\begin{array}{c}0.8 \\
1\end{array}$ & 0.06196 & $\begin{array}{c}0.003 \\
15\end{array}$ & $\begin{array}{c}0.8060 \\
7\end{array}$ & $\begin{array}{c}0.039 \\
08\end{array}$ & $\begin{array}{c}0.0943 \\
8\end{array}$ & 0.001 & 673 & 70 & 600 & 22 & 581 & $\begin{array}{l}1 \\
1\end{array}$ & $3.27 \%$ \\
\hline MB2\#72 & 356 & 738 & $\begin{array}{c}0.4 \\
8\end{array}$ & 0.13109 & $\begin{array}{c}0.002 \\
81\end{array}$ & $\begin{array}{c}5.2619 \\
1\end{array}$ & $\begin{array}{c}0.094 \\
61\end{array}$ & $\begin{array}{c}0.2911 \\
2\end{array}$ & $\begin{array}{c}0.003 \\
40\end{array}$ & 2113 & 38 & 1863 & 15 & 1647 & $\begin{array}{l}1 \\
7\end{array}$ & $28.29 \%$ \\
\hline MB2\#73 & 67 & 92 & $\begin{array}{c}0.7 \\
3\end{array}$ & 0.11685 & $\begin{array}{c}0.003 \\
61\end{array}$ & $\begin{array}{c}5.3706 \\
2\end{array}$ & $\begin{array}{c}0.158 \\
70\end{array}$ & $\begin{array}{c}0.3334 \\
4\end{array}$ & $\begin{array}{c}0.006 \\
25\end{array}$ & 1909 & 28 & 1880 & 25 & 1855 & $\begin{array}{l}3 \\
0\end{array}$ & $2.91 \%$ \\
\hline MB2\#74 & 14 & 47 & $\begin{array}{c}0.3 \\
0\end{array}$ & 0.13114 & $\begin{array}{c}0.005 \\
66\end{array}$ & $\begin{array}{c}6.7053 \\
1\end{array}$ & $\begin{array}{c}0.277 \\
39\end{array}$ & $\begin{array}{c}0.3709 \\
4\end{array}$ & $\begin{array}{c}0.009 \\
76\end{array}$ & 2113 & 38 & 2073 & 37 & 2034 & $\begin{array}{l}4 \\
6\end{array}$ & $3.88 \%$ \\
\hline MB2\#75 & 129 & 192 & $\begin{array}{c}0.6 \\
8\end{array}$ & 0.06204 & $\begin{array}{c}0.003 \\
62\end{array}$ & $\begin{array}{c}0.8955 \\
7\end{array}$ & $\begin{array}{c}0.049 \\
91\end{array}$ & $\begin{array}{c}0.1047 \\
3\end{array}$ & $\begin{array}{c}0.002 \\
34\end{array}$ & 675 & 81 & 649 & 27 & 642 & $\begin{array}{l}1 \\
4\end{array}$ & $1.09 \%$ \\
\hline MB2\#76 & 394 & 617 & $\begin{array}{c}0.6 \\
4\end{array}$ & 0.06330 & $\begin{array}{c}0.004 \\
51\end{array}$ & $\begin{array}{c}0.6713 \\
7\end{array}$ & $\begin{array}{c}0.046 \\
21\end{array}$ & $\begin{array}{c}0.0769 \\
3\end{array}$ & $\begin{array}{c}0.001 \\
40\end{array}$ & 718 & $\begin{array}{c}15 \\
6\end{array}$ & 522 & 28 & 478 & 8 & $9.21 \%$ \\
\hline MB2\#77 & 50 & 118 & $\begin{array}{c}0.4 \\
2\end{array}$ & 0.06306 & $\begin{array}{c}0.004 \\
68\end{array}$ & $\begin{array}{c}0.8026 \\
3\end{array}$ & $\begin{array}{c}0.056 \\
84\end{array}$ & $\begin{array}{c}0.0923 \\
4\end{array}$ & $\begin{array}{c}0.002 \\
52\end{array}$ & 710 & $\begin{array}{c}10 \\
4\end{array}$ & 598 & 32 & 569 & $\begin{array}{l}1 \\
5\end{array}$ & $5.10 \%$ \\
\hline MB2\#78 & 62 & 85 & 0.7 & 0.10021 & $\begin{array}{c}0.013 \\
56\end{array}$ & $\begin{array}{c}1.4486 \\
8\end{array}$ & $\begin{array}{c}0.182 \\
64\end{array}$ & $\begin{array}{c}0.1048 \\
8\end{array}$ & $\begin{array}{c}0.006 \\
18\end{array}$ & 1628 & $\begin{array}{c}14 \\
8\end{array}$ & 909 & 76 & 643 & $\begin{array}{l}3 \\
6\end{array}$ & $41.37 \%$ \\
\hline MB2\#79 & 49 & 45 & $\begin{array}{c}1.1 \\
0\end{array}$ & 0.11893 & $\begin{array}{c}0.004 \\
89\end{array}$ & $\begin{array}{c}6.2106 \\
3\end{array}$ & $\begin{array}{c}0.245 \\
76\end{array}$ & $\begin{array}{c}0.3788 \\
5\end{array}$ & $\begin{array}{c}0.009 \\
25\end{array}$ & 1940 & 38 & 2006 & 35 & 2071 & $\begin{array}{l}4 \\
3\end{array}$ & $-6.33 \%$ \\
\hline MB2\#80 & 44 & 53 & $\begin{array}{c}0.8 \\
3\end{array}$ & 0.05467 & $\begin{array}{c}0.017 \\
64\end{array}$ & $\begin{array}{c}0.6335 \\
6\end{array}$ & $\begin{array}{c}0.200 \\
70\end{array}$ & $\begin{array}{c}0.0840 \\
5\end{array}$ & $\begin{array}{c}0.005 \\
18\end{array}$ & 399 & $\begin{array}{c}59 \\
6\end{array}$ & 498 & $\begin{array}{c}12 \\
5\end{array}$ & 520 & $\begin{array}{l}3 \\
1\end{array}$ & $-4.23 \%$ \\
\hline MB2\#81 & 208 & 135 & $\begin{array}{c}1.5 \\
4\end{array}$ & 0.06611 & $\begin{array}{c}0.003 \\
91\end{array}$ & $\begin{array}{c}0.9200 \\
3\end{array}$ & $\begin{array}{c}0.051 \\
63\end{array}$ & $\begin{array}{c}0.1009 \\
6\end{array}$ & $\begin{array}{c}0.002 \\
37\end{array}$ & 810 & 78 & 662 & 27 & 620 & $\begin{array}{l}1 \\
4\end{array}$ & $6.77 \%$ \\
\hline MB2\#82 & 16 & 107 & $\begin{array}{c}0.1 \\
5\end{array}$ & 0.06162 & $\begin{array}{c}0.004 \\
28\end{array}$ & $\begin{array}{c}0.9374 \\
1\end{array}$ & $\begin{array}{c}0.062 \\
29\end{array}$ & $\begin{array}{c}0.1103 \\
6\end{array}$ & $\begin{array}{c}0.002 \\
87\end{array}$ & 661 & 98 & 672 & 33 & 675 & $\begin{array}{l}1 \\
7\end{array}$ & $-0.44 \%$ \\
\hline
\end{tabular}




\begin{tabular}{|c|c|c|c|c|c|c|c|c|c|c|c|c|c|c|c|c|}
\hline MB2\#83 & 85 & 109 & $\begin{array}{c}0.7 \\
8\end{array}$ & 0.07001 & $\begin{array}{c}0.005 \\
49\end{array}$ & $\begin{array}{c}0.8177 \\
6\end{array}$ & $\begin{array}{c}0.060 \\
82\end{array}$ & $\begin{array}{c}0.0847 \\
4\end{array}$ & $\begin{array}{c}0.002 \\
52\end{array}$ & 929 & $\begin{array}{c}10 \\
4\end{array}$ & 607 & 34 & 524 & $\begin{array}{l}1 \\
5\end{array}$ & $15.84 \%$ \\
\hline MB2\#84 & 90 & 88 & $\begin{array}{c}1.0 \\
2\end{array}$ & 0.12261 & $\begin{array}{c}0.005 \\
75\end{array}$ & $\begin{array}{c}5.4918 \\
0\end{array}$ & $\begin{array}{c}0.245 \\
49\end{array}$ & $\begin{array}{c}0.3249 \\
6\end{array}$ & $\begin{array}{c}0.008 \\
74\end{array}$ & 1995 & 43 & 1899 & 38 & 1814 & $\begin{array}{l}4 \\
3\end{array}$ & $9.98 \%$ \\
\hline MB2\#85 & 26 & 80 & $\begin{array}{c}0.3 \\
3\end{array}$ & 0.05894 & $\begin{array}{c}0.006 \\
43\end{array}$ & $\begin{array}{c}0.8168 \\
1\end{array}$ & $\begin{array}{c}0.085 \\
96\end{array}$ & $\begin{array}{c}0.1005 \\
0\end{array}$ & $\begin{array}{c}0.002 \\
89\end{array}$ & 565 & $\begin{array}{c}24 \\
7\end{array}$ & 606 & 48 & 617 & $\begin{array}{l}1 \\
7\end{array}$ & $-1.78 \%$ \\
\hline MB2\#86 & 6 & 18 & $\begin{array}{c}0.3 \\
3\end{array}$ & 0.22792 & $\begin{array}{c}0.013 \\
88\end{array}$ & $\begin{array}{c}14.162 \\
84\end{array}$ & $\begin{array}{c}0.701 \\
44\end{array}$ & $\begin{array}{c}0.4506 \\
9\end{array}$ & $\begin{array}{c}0.015 \\
96\end{array}$ & 3037 & 10 & 61 & 47 & 2398 & $\begin{array}{l}7 \\
1\end{array}$ & $26.65 \%$ \\
\hline MB2\#87 & 240 & 341 & $\begin{array}{c}0.7 \\
0\end{array}$ & 0.07079 & $\begin{array}{c}0.003 \\
33\end{array}$ & $\begin{array}{c}0.8729 \\
8\end{array}$ & $\begin{array}{c}0.038 \\
84\end{array}$ & $\begin{array}{c}0.0894 \\
7\end{array}$ & $\begin{array}{c}0.001 \\
77\end{array}$ & & & 637 & 21 & 552 & $\begin{array}{l}1 \\
0\end{array}$ & $15.40 \%$ \\
\hline MB2\#88 & 116 & 420 & $\begin{array}{c}0.2 \\
8\end{array}$ & 0.04605 & $\begin{array}{c}0.005 \\
48\end{array}$ & $\begin{array}{c}0.2823 \\
5\end{array}$ & $\begin{array}{c}0.032 \\
25\end{array}$ & $\begin{array}{c}0.0444 \\
7\end{array}$ & $\begin{array}{c}0.001 \\
49\end{array}$ & & $\begin{array}{c}23 \\
6\end{array}$ & 253 & 26 & 280 & 9 & $-9.64 \%$ \\
\hline MB2\#89 & 368 & 312 & $\begin{array}{c}1.1 \\
8\end{array}$ & 0.07407 & $\begin{array}{c}0.003 \\
73\end{array}$ & $\begin{array}{c}1.1462 \\
4\end{array}$ & $\begin{array}{c}0.054 \\
58\end{array}$ & $\begin{array}{c}0.1122 \\
7\end{array}$ & $\begin{array}{c}0.002 \\
40\end{array}$ & 043 & 62 & 775 & 26 & 686 & $\begin{array}{l}1 \\
4\end{array}$ & $12.97 \%$ \\
\hline MB2\#90 & 17 & 21 & $\begin{array}{c}0.7 \\
9\end{array}$ & 0.08888 & $\begin{array}{c}0.025 \\
12\end{array}$ & $\begin{array}{c}3.0782 \\
0\end{array}$ & $\begin{array}{c}0.849 \\
88\end{array}$ & $\begin{array}{c}0.2511 \\
9\end{array}$ & $\begin{array}{c}0.015 \\
24\end{array}$ & 1402 & $\begin{array}{c}61 \\
4\end{array}$ & 1427 & $\begin{array}{c}21 \\
2\end{array}$ & 1445 & $\begin{array}{l}7 \\
8\end{array}$ & $-2.98 \%$ \\
\hline MB2\#91 & 92 & 141 & $\begin{array}{c}0.6 \\
5\end{array}$ & 0.06518 & $\begin{array}{c}0.004 \\
73\end{array}$ & $\begin{array}{c}0.8210 \\
7\end{array}$ & $\begin{array}{c}0.056 \\
63\end{array}$ & $\begin{array}{c}0.0913 \\
9\end{array}$ & $\begin{array}{c}0.002 \\
51\end{array}$ & 780 & 99 & 609 & 32 & 564 & $\begin{array}{l}1 \\
5\end{array}$ & $7.98 \%$ \\
\hline MB2\#92 & 37 & 50 & $\begin{array}{c}0.7 \\
4\end{array}$ & 0.11343 & $\begin{array}{c}0.004 \\
71\end{array}$ & 5.2079 & $\begin{array}{c}0.207 \\
00\end{array}$ & $\begin{array}{c}0.3330 \\
7\end{array}$ & $\begin{array}{c}0.007 \\
88\end{array}$ & 1855 & 39 & 1854 & 34 & 1853 & $\begin{array}{l}3 \\
8\end{array}$ & $0.11 \%$ \\
\hline MB2\#93 & 244 & 317 & $\begin{array}{c}0.7 \\
7\end{array}$ & 0.05522 & $\begin{array}{c}0.016 \\
42\end{array}$ & $\begin{array}{c}0.4037 \\
8\end{array}$ & $\begin{array}{c}0.118 \\
74\end{array}$ & $\begin{array}{c}0.0530 \\
3\end{array}$ & $\begin{array}{c}0.002 \\
32\end{array}$ & 421 & $\begin{array}{c}55 \\
7\end{array}$ & 344 & 86 & 333 & $\begin{array}{l}1 \\
4\end{array}$ & $3.30 \%$ \\
\hline MB2\#94 & 52 & 87 & $\begin{array}{c}0.6 \\
0\end{array}$ & 0.05268 & $\begin{array}{c}0.004 \\
63\end{array}$ & $\begin{array}{c}0.7528 \\
8\end{array}$ & $\begin{array}{c}0.063 \\
72\end{array}$ & $\begin{array}{c}0.1036 \\
9\end{array}$ & $\begin{array}{c}0.003 \\
03\end{array}$ & 315 & $\begin{array}{c}13 \\
9\end{array}$ & 570 & 37 & 636 & $\begin{array}{l}1 \\
8\end{array}$ & $-10.38 \%$ \\
\hline MB2\#95 & 317 & 325 & $\begin{array}{c}0.9 \\
8\end{array}$ & 0.079 & $\begin{array}{c}0.003 \\
89\end{array}$ & $\begin{array}{c}0.9506 \\
8\end{array}$ & $\begin{array}{c}0.044 \\
01\end{array}$ & $\begin{array}{c}0.0872 \\
6\end{array}$ & $\begin{array}{c}0.001 \\
84\end{array}$ & 1173 & 58 & 678 & 23 & 539 & $\begin{array}{l}1 \\
1\end{array}$ & $25.79 \%$ \\
\hline MB2\#96 & 53 & 52 & $\begin{array}{c}1.0 \\
3\end{array}$ & 0.04605 & $\begin{array}{c}0.019 \\
56\end{array}$ & $\begin{array}{c}0.3389 \\
1\end{array}$ & $\begin{array}{c}0.141 \\
72\end{array}$ & $\begin{array}{c}0.0533 \\
8\end{array}$ & $\begin{array}{c}0.003 \\
97\end{array}$ & & $\begin{array}{c}70 \\
3\end{array}$ & 296 & $\begin{array}{c}10 \\
7\end{array}$ & 335 & $\begin{array}{l}2 \\
4\end{array}$ & $-11.64 \%$ \\
\hline MB2\#97 & 154 & 210 & $\begin{array}{c}0.7 \\
3\end{array}$ & 0.09529 & $\begin{array}{c}0.003 \\
64\end{array}$ & $\begin{array}{c}2.7053 \\
3\end{array}$ & $\begin{array}{c}0.097 \\
64\end{array}$ & $\begin{array}{c}0.2059 \\
6\end{array}$ & $\begin{array}{c}0.004 \\
03\end{array}$ & 1534 & 39 & 1330 & 27 & 1207 & $\begin{array}{l}2 \\
2\end{array}$ & $27.09 \%$ \\
\hline MB2\#98 & 114 & 331 & $\begin{array}{c}0.3 \\
4\end{array}$ & 0.06005 & $\begin{array}{c}0.002 \\
39\end{array}$ & $\begin{array}{c}0.7922 \\
2\end{array}$ & $\begin{array}{c}0.030 \\
05\end{array}$ & $\begin{array}{c}0.0957 \\
1\end{array}$ & $\begin{array}{c}0.001 \\
58\end{array}$ & 605 & 54 & 592 & 17 & 589 & 9 & $0.51 \%$ \\
\hline MB2\#99 & 693 & 423 & $\begin{array}{c}1.6 \\
4\end{array}$ & 0.05835 & $\begin{array}{c}0.007 \\
80\end{array}$ & $\begin{array}{c}0.6858 \\
2\end{array}$ & $\begin{array}{c}0.090 \\
24\end{array}$ & $\begin{array}{c}0.0852 \\
4\end{array}$ & $\begin{array}{c}0.001 \\
99\end{array}$ & 543 & $\begin{array}{c}30 \\
4\end{array}$ & 530 & 54 & 527 & $\begin{array}{l}1 \\
2\end{array}$ & $0.57 \%$ \\
\hline MB2\#100 & 82 & 82 & 1.0 & 0.07126 & 0.005 & 0.9228 & 0.064 & 0.0939 & 0.002 & 965 & 95 & 664 & 34 & 579 & 1 & $14.68 \%$ \\
\hline
\end{tabular}




\section{RO2}

\begin{tabular}{|c|c|c|c|c|c|c|c|c|c|c|c|c|c|c|c|c|}
\hline RO2\#01 & 47 & 38 & $\begin{array}{c}1.2 \\
3\end{array}$ & 0.13523 & $\begin{array}{c}0.005 \\
53\end{array}$ & $\begin{array}{c}7.3652 \\
7\end{array}$ & $\begin{array}{c}0.289 \\
21\end{array}$ & $\begin{array}{c}0.3950 \\
8\end{array}$ & $\begin{array}{c}0.010 \\
15\end{array}$ & 2167 & 35 & 2157 & 35 & 2146 & $\begin{array}{l}4 \\
7\end{array}$ & $0.98 \%$ \\
\hline RO2\#02 & 245 & 387 & $\begin{array}{c}0.6 \\
3\end{array}$ & 0.13641 & $\begin{array}{c}0.002 \\
73\end{array}$ & $\begin{array}{c}6.1182 \\
9\end{array}$ & $\begin{array}{c}0.116 \\
10\end{array}$ & $\begin{array}{c}0.3253 \\
5\end{array}$ & $\begin{array}{c}0.004 \\
69\end{array}$ & 2182 & 15 & 1993 & 17 & 1816 & $\begin{array}{l}2 \\
3\end{array}$ & $20.15 \%$ \\
\hline RO2\#03 & 318 & 707 & $\begin{array}{c}0.4 \\
5\end{array}$ & 0.06035 & $\begin{array}{c}0.003 \\
04\end{array}$ & $\begin{array}{c}0.7366 \\
6\end{array}$ & $\begin{array}{c}0.035 \\
62\end{array}$ & $\begin{array}{c}0.0885 \\
4\end{array}$ & $\begin{array}{c}0.001 \\
26\end{array}$ & 616 & 1 & 560 & 21 & 547 & 7 & $2.38 \%$ \\
\hline RO2\#04 & 277 & 536 & $\begin{array}{c}0.5 \\
2\end{array}$ & 0.06737 & $\begin{array}{c}0.004 \\
13\end{array}$ & $\begin{array}{c}0.7317 \\
9\end{array}$ & $\begin{array}{c}0.043 \\
05\end{array}$ & $\begin{array}{c}0.0787 \\
8\end{array}$ & $\begin{array}{c}0.001 \\
35\end{array}$ & & & 558 & 25 & 489 & 8 & $14.11 \%$ \\
\hline RO2\#05 & 68 & 146 & $\begin{array}{c}0.4 \\
6\end{array}$ & 0.12608 & $\begin{array}{c}0.003 \\
02\end{array}$ & $\begin{array}{c}6.9386 \\
5\end{array}$ & $\begin{array}{c}0.159 \\
90\end{array}$ & $\begin{array}{c}0.3991 \\
9\end{array}$ & $\begin{array}{c}0.006 \\
52\end{array}$ & 2044 & 20 & 2104 & 20 & 2165 & $\begin{array}{l}3 \\
0\end{array}$ & $-5.59 \%$ \\
\hline RO2\#06 & 63 & 53 & $\begin{array}{c}1.2 \\
0\end{array}$ & 0.07199 & $\begin{array}{c}0.015 \\
09\end{array}$ & $\begin{array}{c}0.9288 \\
6\end{array}$ & $\begin{array}{c}0.190 \\
37\end{array}$ & $\begin{array}{c}0.0935 \\
8\end{array}$ & $\begin{array}{c}0.004 \\
13\end{array}$ & 986 & $\begin{array}{c}46 \\
6\end{array}$ & 667 & $\begin{array}{c}10 \\
0\end{array}$ & 577 & $\begin{array}{l}2 \\
4\end{array}$ & $15.60 \%$ \\
\hline RO2\#07 & 70 & 90 & $\begin{array}{c}0.7 \\
8\end{array}$ & 0.06093 & $\begin{array}{c}0.009 \\
49\end{array}$ & $\begin{array}{c}0.7933 \\
7\end{array}$ & $\begin{array}{c}0.120 \\
68\end{array}$ & $\begin{array}{c}0.0944 \\
3\end{array}$ & $\begin{array}{c}0.003 \\
16\end{array}$ & 637 & $\begin{array}{c}34 \\
9\end{array}$ & 593 & 68 & 582 & $\begin{array}{l}1 \\
9\end{array}$ & $1.89 \%$ \\
\hline RO2\#08 & 45 & 75 & $\begin{array}{c}0.6 \\
1\end{array}$ & 0.07040 & $\begin{array}{c}0.005 \\
08\end{array}$ & $\begin{array}{c}1.0710 \\
0\end{array}$ & $\begin{array}{c}0.073 \\
58\end{array}$ & $\begin{array}{c}0.1103 \\
4\end{array}$ & $\begin{array}{c}0.003 \\
10\end{array}$ & 940 & 95 & 739 & 36 & 675 & $\begin{array}{l}1 \\
8\end{array}$ & $9.48 \%$ \\
\hline RO2\#09 & 108 & 240 & $\begin{array}{c}0.4 \\
5\end{array}$ & 0.07345 & $\begin{array}{c}0.004 \\
58\end{array}$ & 1.1370 & $\begin{array}{c}0.067 \\
21\end{array}$ & $\begin{array}{c}0.1122 \\
8\end{array}$ & $\begin{array}{c}0.002 \\
87\end{array}$ & 1026 & 78 & 771 & 32 & 686 & $\begin{array}{l}1 \\
7\end{array}$ & $12.39 \%$ \\
\hline RO2\#10 & 62 & 90 & $\begin{array}{c}0.6 \\
8\end{array}$ & 0.11705 & $\begin{array}{c}0.003 \\
80\end{array}$ & $\begin{array}{c}5.4614 \\
0\end{array}$ & $\begin{array}{c}0.169 \\
29\end{array}$ & $\begin{array}{c}0.3384 \\
2\end{array}$ & $\begin{array}{c}0.006 \\
65\end{array}$ & 1912 & 29 & 1895 & 27 & 1879 & $\begin{array}{l}3 \\
2\end{array}$ & $1.76 \%$ \\
\hline RO2\#11 & 250 & 297 & $\begin{array}{c}0.8 \\
4\end{array}$ & 0.06188 & $\begin{array}{c}0.002 \\
62\end{array}$ & $\begin{array}{c}0.8103 \\
1\end{array}$ & $\begin{array}{c}0.032 \\
63\end{array}$ & $\begin{array}{c}0.0949 \\
7\end{array}$ & $\begin{array}{c}0.001 \\
66\end{array}$ & 670 & 56 & 603 & 18 & 585 & $\begin{array}{l}1 \\
0\end{array}$ & $3.08 \%$ \\
\hline RO2\#12 & 76 & 100 & $\begin{array}{c}0.7 \\
6\end{array}$ & 0.05603 & $\begin{array}{c}0.004 \\
47\end{array}$ & $\begin{array}{c}0.7086 \\
5\end{array}$ & $\begin{array}{c}0.054 \\
21\end{array}$ & $\begin{array}{c}0.0917 \\
3\end{array}$ & $\begin{array}{c}0.002 \\
55\end{array}$ & 454 & $\begin{array}{c}12 \\
1\end{array}$ & 544 & 32 & 566 & $\begin{array}{l}1 \\
5\end{array}$ & $-3.89 \%$ \\
\hline RO2\#13 & 197 & 360 & $\begin{array}{c}0.5 \\
5\end{array}$ & 0.10653 & $\begin{array}{c}0.003 \\
09\end{array}$ & $\begin{array}{c}4.1843 \\
6\end{array}$ & $\begin{array}{c}0.108 \\
39\end{array}$ & $\begin{array}{c}0.2848 \\
7\end{array}$ & $\begin{array}{c}0.003 \\
70\end{array}$ & 1741 & 54 & 1671 & 21 & 1616 & $\begin{array}{l}1 \\
9\end{array}$ & $7.74 \%$ \\
\hline RO2\#14 & 149 & 183 & $\begin{array}{c}0.8 \\
1\end{array}$ & 0.08042 & $\begin{array}{c}0.010 \\
49\end{array}$ & $\begin{array}{c}1.2119 \\
9\end{array}$ & $\begin{array}{c}0.153 \\
18\end{array}$ & $\begin{array}{c}0.1093 \\
0\end{array}$ & $\begin{array}{c}0.003 \\
51\end{array}$ & 1207 & $\begin{array}{c}27 \\
1\end{array}$ & 806 & 70 & 669 & $\begin{array}{l}2 \\
0\end{array}$ & $20.48 \%$ \\
\hline RO2\#15 & 190 & 91 & $\begin{array}{c}2.0 \\
9\end{array}$ & 0.11788 & $\begin{array}{c}0.003 \\
62\end{array}$ & $\begin{array}{c}5.8486 \\
1\end{array}$ & $\begin{array}{c}0.172 \\
24\end{array}$ & $\begin{array}{c}0.3598 \\
3\end{array}$ & $\begin{array}{c}0.006 \\
84\end{array}$ & 1924 & 27 & 1954 & 26 & 1981 & $\begin{array}{l}3 \\
2\end{array}$ & $-2.88 \%$ \\
\hline$R O 2 \# 16$ & 87 & 85 & $\begin{array}{c}1.0 \\
2\end{array}$ & 0.08036 & $\begin{array}{c}0.007 \\
64\end{array}$ & $\begin{array}{c}1.2329 \\
5\end{array}$ & $\begin{array}{c}0.110 \\
95\end{array}$ & $\begin{array}{c}0.1112 \\
7\end{array}$ & $\begin{array}{c}0.004 \\
19\end{array}$ & 1206 & $\begin{array}{c}11 \\
8\end{array}$ & 816 & 50 & 680 & $\begin{array}{l}2 \\
4\end{array}$ & $20.00 \%$ \\
\hline RO2\#17 & 37 & 45 & 0.8 & 0.26896 & 0.006 & 23.588 & 0.584 & 0.6360 & 0.014 & 3300 & 17 & 3252 & 24 & 3173 & 5 & $4.00 \%$ \\
\hline
\end{tabular}




\begin{tabular}{|c|c|c|c|c|c|c|c|c|c|c|c|c|c|c|c|c|}
\hline & & & 2 & & 66 & 42 & 77 & 5 & 22 & & & & & & 6 & \\
\hline RO2\#18 & 135 & 172 & $\begin{array}{c}0.7 \\
8\end{array}$ & 0.17843 & $\begin{array}{c}0.003 \\
61\end{array}$ & $\begin{array}{c}13.630 \\
62\end{array}$ & $\begin{array}{c}0.271 \\
16\end{array}$ & $\begin{array}{c}0.5540 \\
2\end{array}$ & $\begin{array}{c}0.009 \\
20\end{array}$ & 2638 & 15 & 2724 & 19 & 2842 & $\begin{array}{l}3 \\
8\end{array}$ & $-7.18 \%$ \\
\hline RO2\#19 & 380 & 138 & $\begin{array}{c}2.7 \\
6\end{array}$ & 0.06783 & $\begin{array}{c}0.004 \\
48\end{array}$ & $\begin{array}{c}1.0634 \\
2\end{array}$ & $\begin{array}{c}0.066 \\
72\end{array}$ & $\begin{array}{c}0.1136 \\
9\end{array}$ & $\begin{array}{c}0.002 \\
98\end{array}$ & 863 & 87 & 736 & 33 & 694 & $\begin{array}{l}1 \\
7\end{array}$ & $6.05 \%$ \\
\hline$R O 2 \# 20$ & 99 & 87 & $\begin{array}{c}1.1 \\
4\end{array}$ & 0.08376 & $\begin{array}{c}0.010 \\
28\end{array}$ & $\begin{array}{c}1.2025 \\
6\end{array}$ & $\begin{array}{c}0.139 \\
29\end{array}$ & $\begin{array}{c}0.1041 \\
2\end{array}$ & $\begin{array}{c}0.005 \\
11\end{array}$ & 1287 & $\begin{array}{c}15 \\
0\end{array}$ & & 64 & 639 & $\begin{array}{l}3 \\
0\end{array}$ & $25.51 \%$ \\
\hline RO2\#21 & 88 & 38 & $\begin{array}{c}2.3 \\
0\end{array}$ & 0.10122 & $\begin{array}{c}0.021 \\
53\end{array}$ & $\begin{array}{c}2.7555 \\
9\end{array}$ & $\begin{array}{c}0.570 \\
46\end{array}$ & $\begin{array}{c}0.1974 \\
5\end{array}$ & $\begin{array}{c}0.009 \\
68\end{array}$ & & & & $\begin{array}{c}15 \\
4\end{array}$ & 1162 & $\begin{array}{l}5 \\
2\end{array}$ & $41.74 \%$ \\
\hline RO2\#22 & 96 & 99 & $\begin{array}{c}0.9 \\
7\end{array}$ & 0.09710 & $\begin{array}{c}0.006 \\
44\end{array}$ & $\begin{array}{c}1.8728 \\
4\end{array}$ & $\begin{array}{c}0.116 \\
25\end{array}$ & $\begin{array}{c}0.1398 \\
7\end{array}$ & $\begin{array}{c}0.004 \\
29\end{array}$ & 69 & 71 & 1072 & 41 & 844 & $\begin{array}{l}2 \\
4\end{array}$ & $27.01 \%$ \\
\hline RO2\#23 & 114 & 114 & $\begin{array}{c}1.0 \\
0\end{array}$ & 0.18770 & $\begin{array}{c}0.010 \\
03\end{array}$ & $\begin{array}{c}7.4951 \\
7\end{array}$ & $\begin{array}{c}0.370 \\
34\end{array}$ & $\begin{array}{c}0.2895 \\
7\end{array}$ & $\begin{array}{c}0.010 \\
09\end{array}$ & 2722 & 40 & 2172 & 44 & 1639 & $\begin{array}{l}5 \\
0\end{array}$ & $66.08 \%$ \\
\hline RO2\#24 & 203 & 376 & $\begin{array}{c}0.5 \\
4\end{array}$ & 0.07347 & $\begin{array}{c}0.005 \\
32\end{array}$ & $\begin{array}{c}0.8425 \\
8\end{array}$ & $\begin{array}{c}0.058 \\
54\end{array}$ & $\begin{array}{c}0.0831 \\
8\end{array}$ & $\begin{array}{c}0.001 \\
71\end{array}$ & 1027 & $\begin{array}{c}15 \\
1\end{array}$ & 621 & 32 & 515 & $\begin{array}{l}1 \\
0\end{array}$ & $20.58 \%$ \\
\hline RO2\#25 & 17 & 30 & $\begin{array}{c}0.5 \\
6\end{array}$ & 0.13984 & $\begin{array}{c}0.007 \\
26\end{array}$ & $\begin{array}{c}6.6490 \\
1\end{array}$ & $\begin{array}{c}0.328 \\
36\end{array}$ & $\begin{array}{c}0.3447 \\
8\end{array}$ & $\begin{array}{c}0.010 \\
79\end{array}$ & 2225 & 45 & 2066 & 44 & 1910 & $\begin{array}{l}5 \\
2\end{array}$ & $16.49 \%$ \\
\hline RO2\#26 & 192 & 297 & $\begin{array}{c}0.6 \\
5\end{array}$ & 0.06207 & $\begin{array}{c}0.004 \\
36\end{array}$ & $\begin{array}{c}0.9028 \\
7\end{array}$ & $\begin{array}{c}0.061 \\
20\end{array}$ & $\begin{array}{c}0.1055 \\
0\end{array}$ & $\begin{array}{c}0.001 \\
92\end{array}$ & 676 & $\begin{array}{c}15 \\
5\end{array}$ & 653 & 33 & 647 & $\begin{array}{l}1 \\
1\end{array}$ & $0.93 \%$ \\
\hline RO2\#27 & 412 & 277 & $\begin{array}{c}1.4 \\
9\end{array}$ & 0.07527 & $\begin{array}{c}0.003 \\
58\end{array}$ & $\begin{array}{c}1.0036 \\
6\end{array}$ & $\begin{array}{c}0.045 \\
11\end{array}$ & $\begin{array}{c}0.0967 \\
0\end{array}$ & $\begin{array}{c}0.001 \\
97\end{array}$ & 1076 & 58 & 706 & 23 & 595 & $\begin{array}{l}1 \\
2\end{array}$ & $18.66 \%$ \\
\hline$R O 2 \# 28$ & 88 & 79 & $\begin{array}{c}1.1 \\
1\end{array}$ & 0.08034 & $\begin{array}{c}0.018 \\
89\end{array}$ & $\begin{array}{c}0.8175 \\
6\end{array}$ & $\begin{array}{c}0.188 \\
24\end{array}$ & $\begin{array}{c}0.0738 \\
1\end{array}$ & $\begin{array}{c}0.003 \\
53\end{array}$ & 1205 & $\begin{array}{c}52 \\
1\end{array}$ & 607 & $\begin{array}{c}10 \\
5\end{array}$ & 459 & $\begin{array}{l}2 \\
1\end{array}$ & $32.24 \%$ \\
\hline RO2\#29 & 61 & 40 & $\begin{array}{c}1.5 \\
2\end{array}$ & 0.18624 & $\begin{array}{c}0.009 \\
81\end{array}$ & $\begin{array}{c}10.911 \\
45\end{array}$ & $\begin{array}{c}0.549 \\
02\end{array}$ & $\begin{array}{c}0.4248 \\
5\end{array}$ & $\begin{array}{c}0.015 \\
55\end{array}$ & 2709 & 40 & 2516 & 47 & 2282 & $\begin{array}{l}7 \\
0\end{array}$ & $18.71 \%$ \\
\hline RO2\#30 & 67 & 49 & $\begin{array}{c}1.3 \\
7\end{array}$ & 0.08613 & $\begin{array}{c}0.027 \\
00\end{array}$ & $\begin{array}{c}0.7559 \\
7\end{array}$ & $\begin{array}{c}0.231 \\
29\end{array}$ & $\begin{array}{c}0.0636 \\
6\end{array}$ & $\begin{array}{c}0.004 \\
34\end{array}$ & 1341 & $\begin{array}{c}68 \\
3\end{array}$ & 572 & $\begin{array}{c}13 \\
4\end{array}$ & 398 & $\begin{array}{l}2 \\
6\end{array}$ & $43.72 \%$ \\
\hline RO2\#31 & 117 & 176 & $\begin{array}{c}0.6 \\
6\end{array}$ & 0.07826 & $\begin{array}{c}0.004 \\
94\end{array}$ & $\begin{array}{c}1.0976 \\
2\end{array}$ & $\begin{array}{c}0.065 \\
45\end{array}$ & $\begin{array}{c}0.1016 \\
9\end{array}$ & $\begin{array}{c}0.002 \\
66\end{array}$ & 1153 & 77 & 752 & 32 & 624 & $\begin{array}{l}1 \\
6\end{array}$ & $20.51 \%$ \\
\hline RO2\#32 & 54 & 51 & $\begin{array}{c}1.0 \\
4\end{array}$ & 0.11688 & $\begin{array}{c}0.008 \\
94\end{array}$ & $\begin{array}{c}2.3232 \\
2\end{array}$ & $\begin{array}{c}0.164 \\
49\end{array}$ & $\begin{array}{c}0.1441 \\
2\end{array}$ & $\begin{array}{c}0.005 \\
44\end{array}$ & 1909 & 74 & 1219 & 50 & 868 & $\begin{array}{l}3 \\
1\end{array}$ & $40.44 \%$ \\
\hline RO2\#33 & 2 & 52 & $\begin{array}{c}0.0 \\
3\end{array}$ & 0.05645 & $\begin{array}{c}0.005 \\
30\end{array}$ & $\begin{array}{c}1.0703 \\
3\end{array}$ & $\begin{array}{c}0.096 \\
80\end{array}$ & $\begin{array}{c}0.1374 \\
8\end{array}$ & $\begin{array}{c}0.004 \\
56\end{array}$ & 470 & $\begin{array}{c}14 \\
2\end{array}$ & 739 & 47 & 830 & $\begin{array}{l}2 \\
6\end{array}$ & $-10.96 \%$ \\
\hline RO2\#34 & 133 & 286 & $\begin{array}{c}0.4 \\
6\end{array}$ & 0.08017 & $\begin{array}{c}0.003 \\
31\end{array}$ & $\begin{array}{c}1.0363 \\
6\end{array}$ & $\begin{array}{c}0.040 \\
21\end{array}$ & $\begin{array}{c}0.0937 \\
3\end{array}$ & $\begin{array}{c}0.001 \\
76\end{array}$ & 1201 & 47 & 722 & 20 & 578 & $\begin{array}{l}1 \\
0\end{array}$ & $24.91 \%$ \\
\hline
\end{tabular}




\begin{tabular}{|c|c|c|c|c|c|c|c|c|c|c|c|c|c|c|c|c|}
\hline RO2\#35 & 108 & 179 & $\begin{array}{c}0.6 \\
0\end{array}$ & 0.11173 & $\begin{array}{c}0.002 \\
77\end{array}$ & $\begin{array}{c}4.7507 \\
1\end{array}$ & $\begin{array}{c}0.112 \\
32\end{array}$ & $\begin{array}{c}0.3082 \\
8\end{array}$ & $\begin{array}{c}0.004 \\
83\end{array}$ & 1828 & 22 & 1776 & 20 & 1732 & 2 & $5.54 \%$ \\
\hline RO2\#36 & 255 & 306 & $\begin{array}{c}0.8 \\
3\end{array}$ & 0.07499 & $\begin{array}{c}0.006 \\
78\end{array}$ & $\begin{array}{c}0.8421 \\
5\end{array}$ & $\begin{array}{c}0.073 \\
67\end{array}$ & $\begin{array}{c}0.0814 \\
4\end{array}$ & $\begin{array}{c}0.001 \\
84\end{array}$ & 1068 & $\begin{array}{c}18 \\
8\end{array}$ & 620 & 41 & 505 & $\begin{array}{l}1 \\
1\end{array}$ & $22.77 \%$ \\
\hline RO2\#37 & 206 & 186 & $\begin{array}{c}1.1 \\
1\end{array}$ & 0.08385 & $\begin{array}{c}0.005 \\
70\end{array}$ & $\begin{array}{c}1.0444 \\
9\end{array}$ & $\begin{array}{c}0.066 \\
64\end{array}$ & $\begin{array}{c}0.0903 \\
2\end{array}$ & $\begin{array}{c}0.002 \\
58\end{array}$ & 1289 & 80 & 726 & 33 & 557 & $\begin{array}{l}1 \\
5\end{array}$ & $30.34 \%$ \\
\hline RO2\#38 & 200 & 325 & $\begin{array}{c}0.6 \\
1\end{array}$ & 0.07042 & $\begin{array}{c}0.004 \\
94\end{array}$ & $\begin{array}{c}0.9922 \\
5\end{array}$ & $\begin{array}{c}0.066 \\
06\end{array}$ & $\begin{array}{c}0.1021 \\
6\end{array}$ & $\begin{array}{c}0.002 \\
83\end{array}$ & 941 & & & 34 & 627 & $\begin{array}{l}1 \\
7\end{array}$ & $11.64 \%$ \\
\hline RO2\#39 & 160 & 179 & $\begin{array}{c}0.9 \\
0\end{array}$ & 0.09386 & $\begin{array}{c}0.007 \\
37\end{array}$ & $\begin{array}{c}1.2095 \\
9\end{array}$ & $\begin{array}{c}0.088 \\
39\end{array}$ & $\begin{array}{c}0.0934 \\
3\end{array}$ & $\begin{array}{c}0.003 \\
23\end{array}$ & & & & 41 & 576 & 1 & $39.76 \%$ \\
\hline RO2\#40 & 81 & 59 & $\begin{array}{c}1.3 \\
7\end{array}$ & 0.18131 & $\begin{array}{c}0.012 \\
90\end{array}$ & $\begin{array}{c}5.7947 \\
4\end{array}$ & $\begin{array}{c}0.376 \\
00\end{array}$ & $\begin{array}{c}0.2317 \\
2\end{array}$ & $\begin{array}{c}0.010 \\
15\end{array}$ & 2665 & 54 & 1946 & 56 & 1344 & 3 & $98.29 \%$ \\
\hline RO2\#41 & 195 & 278 & $\begin{array}{c}0.7 \\
0\end{array}$ & 0.08281 & $\begin{array}{c}0.002 \\
92\end{array}$ & $\begin{array}{c}2.2862 \\
3\end{array}$ & $\begin{array}{c}0.076 \\
54\end{array}$ & $\begin{array}{c}0.2001 \\
5\end{array}$ & $\begin{array}{c}0.003 \\
53\end{array}$ & 1265 & 38 & 1208 & 24 & 1176 & $\begin{array}{l}1 \\
9\end{array}$ & $7.57 \%$ \\
\hline RO2\#42 & 203 & 166 & $\begin{array}{c}1.2 \\
2\end{array}$ & 0.07339 & $\begin{array}{c}0.016 \\
39\end{array}$ & $\begin{array}{c}0.9506 \\
7\end{array}$ & $\begin{array}{c}0.208 \\
02\end{array}$ & $\begin{array}{c}0.0939 \\
4\end{array}$ & $\begin{array}{c}0.004 \\
18\end{array}$ & 1025 & $\begin{array}{c}49 \\
5\end{array}$ & 678 & $\begin{array}{c}10 \\
8\end{array}$ & 579 & $J$ & $17.10 \%$ \\
\hline RO2\#43 & 174 & 85 & $\begin{array}{c}2.0 \\
5\end{array}$ & 0.07390 & $\begin{array}{c}0.025 \\
09\end{array}$ & $\begin{array}{c}1.6174 \\
0\end{array}$ & $\begin{array}{c}0.540 \\
33\end{array}$ & $\begin{array}{c}0.1587 \\
3\end{array}$ & $\begin{array}{c}0.009 \\
60\end{array}$ & 1039 & $\begin{array}{c}71 \\
4\end{array}$ & 977 & $\begin{array}{c}21 \\
0\end{array}$ & 950 & 3 & $2.84 \%$ \\
\hline RO2\#44 & 100 & 57 & $\begin{array}{c}1.7 \\
5\end{array}$ & 0.11641 & $\begin{array}{c}0.004 \\
56\end{array}$ & $\begin{array}{c}5.4583 \\
5\end{array}$ & $\begin{array}{c}0.204 \\
62\end{array}$ & $\begin{array}{c}0.3399 \\
2\end{array}$ & $\begin{array}{c}0.007 \\
79\end{array}$ & 1902 & 36 & 1894 & 32 & 1886 & $\begin{array}{l}3 \\
7\end{array}$ & $0.85 \%$ \\
\hline RO2\#45 & 63 & 57 & $\begin{array}{c}1.1 \\
2\end{array}$ & 0.16958 & $\begin{array}{c}0.014 \\
82\end{array}$ & $\begin{array}{c}6.6702 \\
7\end{array}$ & $\begin{array}{c}0.542 \\
39\end{array}$ & $\begin{array}{c}0.2851 \\
6\end{array}$ & $\begin{array}{c}0.015 \\
24\end{array}$ & 2554 & 70 & 2069 & 72 & 1617 & $\begin{array}{l}7 \\
6\end{array}$ & $57.95 \%$ \\
\hline RO2\#46 & 112 & 74 & $\begin{array}{c}1.5 \\
2\end{array}$ & 0.06681 & $\begin{array}{c}0.005 \\
80\end{array}$ & $\begin{array}{c}0.9757 \\
0\end{array}$ & $\begin{array}{c}0.080 \\
80\end{array}$ & $\begin{array}{c}0.1058 \\
8\end{array}$ & $\begin{array}{c}0.003 \\
44\end{array}$ & 832 & $\begin{array}{c}11 \\
9\end{array}$ & 691 & 42 & 649 & $\begin{array}{l}2 \\
0\end{array}$ & $6.47 \%$ \\
\hline RO2\#47 & 61 & 49 & $\begin{array}{c}1.2 \\
4\end{array}$ & 0.089 & $\begin{array}{c}0.027 \\
68\end{array}$ & $\begin{array}{c}0.8825 \\
4\end{array}$ & $\begin{array}{c}0.264 \\
89\end{array}$ & $\begin{array}{c}0.0712 \\
8\end{array}$ & $\begin{array}{c}0.005 \\
01\end{array}$ & 1421 & $\begin{array}{c}67 \\
0\end{array}$ & 642 & $\begin{array}{c}14 \\
3\end{array}$ & 444 & $\begin{array}{l}3 \\
0\end{array}$ & $44.59 \%$ \\
\hline RO2\#48 & 93 & 96 & $\begin{array}{c}0.9 \\
7\end{array}$ & 0.05939 & $\begin{array}{c}0.004 \\
48\end{array}$ & $\begin{array}{c}0.7857 \\
9\end{array}$ & $\begin{array}{c}0.056 \\
50\end{array}$ & $\begin{array}{c}0.0959 \\
2\end{array}$ & $\begin{array}{c}0.002 \\
67\end{array}$ & 581 & $\begin{array}{c}10 \\
8\end{array}$ & 589 & 32 & 590 & $\begin{array}{l}1 \\
6\end{array}$ & $-0.17 \%$ \\
\hline RO2\#49 & 115 & 109 & $\begin{array}{c}1.0 \\
6\end{array}$ & 0.09406 & $\begin{array}{c}0.007 \\
76\end{array}$ & $\begin{array}{c}1.5750 \\
2\end{array}$ & $\begin{array}{c}0.121 \\
68\end{array}$ & $\begin{array}{c}0.1213 \\
9\end{array}$ & $\begin{array}{c}0.004 \\
40\end{array}$ & 1509 & 92 & 960 & 48 & 739 & $\begin{array}{l}2 \\
5\end{array}$ & $29.91 \%$ \\
\hline RO2\#50 & 240 & 493 & $\begin{array}{c}0.4 \\
9\end{array}$ & 0.05221 & $\begin{array}{c}0.007 \\
93\end{array}$ & $\begin{array}{c}0.5165 \\
9\end{array}$ & $\begin{array}{c}0.077 \\
28\end{array}$ & $\begin{array}{c}0.0717 \\
7\end{array}$ & $\begin{array}{c}0.001 \\
89\end{array}$ & 294 & $\begin{array}{c}31 \\
9\end{array}$ & 423 & 52 & 447 & $\begin{array}{l}1 \\
1\end{array}$ & $-5.37 \%$ \\
\hline RO2\#51 & 290 & 215 & $\begin{array}{c}1.3 \\
5\end{array}$ & 0.07090 & $\begin{array}{c}0.014 \\
09\end{array}$ & $\begin{array}{c}0.8748 \\
7\end{array}$ & $\begin{array}{c}0.170 \\
30\end{array}$ & $\begin{array}{c}0.0894 \\
9\end{array}$ & $\begin{array}{c}0.003 \\
56\end{array}$ & 955 & $\begin{array}{c}44 \\
0\end{array}$ & 638 & 92 & 553 & $\begin{array}{l}2 \\
1\end{array}$ & $15.37 \%$ \\
\hline RO2\#52 & 109 & 197 & 0.5 & 0.06999 & 0.003 & 0.9334 & 0.047 & 0.0966 & 0.002 & 928 & 68 & 669 & 25 & 595 & 1 & $12.44 \%$ \\
\hline
\end{tabular}




\begin{tabular}{|c|c|c|c|c|c|c|c|c|c|c|c|c|c|c|c|c|}
\hline & & & 5 & & 74 & 2 & 25 & 7 & 11 & & & & & & 2 & \\
\hline RO2\#53 & 49 & 96 & $\begin{array}{c}0.5 \\
1\end{array}$ & 0.13280 & $\begin{array}{c}0.003 \\
56\end{array}$ & $\begin{array}{c}7.3031 \\
3\end{array}$ & $\begin{array}{c}0.188 \\
30\end{array}$ & $\begin{array}{c}0.3986 \\
3\end{array}$ & $\begin{array}{c}0.007 \\
15\end{array}$ & 2135 & 22 & 2149 & 23 & 2163 & $\begin{array}{l}3 \\
3\end{array}$ & $-1.29 \%$ \\
\hline RO2\#54 & 214 & 238 & $\begin{array}{c}0.9 \\
0\end{array}$ & 0.08258 & $\begin{array}{c}0.005 \\
64\end{array}$ & $\begin{array}{c}0.9739 \\
2\end{array}$ & $\begin{array}{c}0.062 \\
44\end{array}$ & $\begin{array}{c}0.0854 \\
9\end{array}$ & $\begin{array}{c}0.002 \\
45\end{array}$ & 1259 & 81 & 690 & 32 & 529 & $\begin{array}{l}1 \\
5\end{array}$ & $30.43 \%$ \\
\hline RO2\#55 & 393 & 411 & $\begin{array}{c}0.9 \\
6\end{array}$ & 0.07921 & $\begin{array}{c}0.005 \\
37\end{array}$ & $\begin{array}{c}1.1089 \\
0\end{array}$ & $\begin{array}{c}0.070 \\
95\end{array}$ & $\begin{array}{c}0.1014 \\
7\end{array}$ & $\begin{array}{c}0.002 \\
85\end{array}$ & 1177 & 82 & & 34 & 623 & $\begin{array}{l}1 \\
7\end{array}$ & $21.67 \%$ \\
\hline RO2\#56 & 106 & 189 & $\begin{array}{c}0.5 \\
6\end{array}$ & 0.04742 & $\begin{array}{c}0.011 \\
09\end{array}$ & $\begin{array}{c}0.6024 \\
5\end{array}$ & $\begin{array}{c}0.138 \\
77\end{array}$ & $\begin{array}{c}0.0921 \\
5\end{array}$ & $\begin{array}{c}0.003 \\
70\end{array}$ & 70 & & & 88 & 568 & $\begin{array}{l}2 \\
2\end{array}$ & $-15.67 \%$ \\
\hline RO2\#57 & 163 & 251 & $\begin{array}{c}0.6 \\
5\end{array}$ & 0.06445 & $\begin{array}{c}0.004 \\
57\end{array}$ & $\begin{array}{c}0.9152 \\
0\end{array}$ & $\begin{array}{c}0.061 \\
86\end{array}$ & $\begin{array}{c}0.1029 \\
3\end{array}$ & $\begin{array}{c}0.002 \\
77\end{array}$ & & 97 & 660 & 33 & 632 & $\begin{array}{l}1 \\
6\end{array}$ & $4.43 \%$ \\
\hline RO2\#58 & 57 & 108 & $\begin{array}{c}0.5 \\
2\end{array}$ & 0.14187 & $\begin{array}{c}0.003 \\
51\end{array}$ & $\begin{array}{c}7.8330 \\
3\end{array}$ & $\begin{array}{c}0.186 \\
06\end{array}$ & $\begin{array}{c}0.4002 \\
0\end{array}$ & $\begin{array}{c}0.006 \\
88\end{array}$ & 2250 & 20 & 2212 & 21 & 2170 & $\begin{array}{l}3 \\
2\end{array}$ & $3.69 \%$ \\
\hline RO2\#59 & 126 & 598 & $\begin{array}{c}0.2 \\
1\end{array}$ & 0.06073 & $\begin{array}{c}0.002 \\
86\end{array}$ & $\begin{array}{c}0.7422 \\
4\end{array}$ & $\begin{array}{c}0.033 \\
15\end{array}$ & $\begin{array}{c}0.0886 \\
4\end{array}$ & $\begin{array}{c}0.001 \\
31\end{array}$ & 630 & $\begin{array}{c}10 \\
4\end{array}$ & 564 & 19 & 547 & 8 & $3.11 \%$ \\
\hline RO2\#60 & 150 & 259 & $\begin{array}{c}0.5 \\
8\end{array}$ & 0.07352 & $\begin{array}{c}0.004 \\
08\end{array}$ & $\begin{array}{c}0.8756 \\
3\end{array}$ & $\begin{array}{c}0.045 \\
90\end{array}$ & $\begin{array}{c}0.0863 \\
3\end{array}$ & $\begin{array}{c}0.001 \\
98\end{array}$ & 1028 & 69 & 639 & 25 & 534 & $\begin{array}{l}1 \\
2\end{array}$ & $19.66 \%$ \\
\hline RO2\#61 & 41 & 43 & $\begin{array}{c}0.9 \\
4\end{array}$ & 0.16495 & $\begin{array}{c}0.005 \\
85\end{array}$ & $\begin{array}{c}8.8082 \\
5\end{array}$ & $\begin{array}{c}0.297 \\
81\end{array}$ & $\begin{array}{c}0.3870 \\
4\end{array}$ & $\begin{array}{c}0.009 \\
29\end{array}$ & 2507 & 28 & 2318 & 31 & 2109 & $\begin{array}{l}4 \\
3\end{array}$ & $18.87 \%$ \\
\hline RO2\#62 & 213 & 315 & $\begin{array}{c}0.6 \\
8\end{array}$ & 0.12004 & $\begin{array}{c}0.004 \\
18\end{array}$ & $\begin{array}{c}4.6845 \\
1\end{array}$ & $\begin{array}{c}0.148 \\
32\end{array}$ & $\begin{array}{c}0.2830 \\
3\end{array}$ & $\begin{array}{c}0.004 \\
12\end{array}$ & 1957 & 64 & 1764 & 26 & 1607 & $\begin{array}{l}2 \\
1\end{array}$ & $21.78 \%$ \\
\hline RO2\#63 & 368 & 786 & $\begin{array}{c}0.4 \\
7\end{array}$ & 0.05837 & $\begin{array}{c}0.003 \\
13\end{array}$ & $\begin{array}{c}0.6839 \\
7\end{array}$ & $\begin{array}{c}0.035 \\
45\end{array}$ & $\begin{array}{c}0.0849 \\
9\end{array}$ & $\begin{array}{c}0.001 \\
19\end{array}$ & 544 & $\begin{array}{c}12 \\
1\end{array}$ & 529 & 21 & 526 & 7 & $0.57 \%$ \\
\hline RO2\#64 & 76 & 38 & $\begin{array}{c}2.0 \\
1\end{array}$ & 0. & $\begin{array}{c}0.005 \\
93\end{array}$ & $\begin{array}{c}5.4560 \\
8\end{array}$ & $\begin{array}{c}0.247 \\
62\end{array}$ & $\begin{array}{c}0.3184 \\
6\end{array}$ & $\begin{array}{c}0.008 \\
76\end{array}$ & 2017 & 43 & 1894 & 39 & 1782 & $\begin{array}{l}4 \\
3\end{array}$ & $13.19 \%$ \\
\hline RO2\#65 & 83 & 170 & $\begin{array}{c}0.4 \\
9\end{array}$ & 0.06281 & $\begin{array}{c}0.003 \\
48\end{array}$ & $\begin{array}{c}0.8224 \\
4\end{array}$ & $\begin{array}{c}0.043 \\
35\end{array}$ & $\begin{array}{c}0.0949 \\
0\end{array}$ & $\begin{array}{c}0.002 \\
06\end{array}$ & 702 & 75 & 609 & 24 & 584 & $\begin{array}{l}1 \\
2\end{array}$ & $4.28 \%$ \\
\hline RO2\#66 & 134 & 463 & $\begin{array}{c}0.2 \\
9\end{array}$ & 0.08312 & $\begin{array}{c}0.005 \\
02\end{array}$ & $\begin{array}{c}1.7412 \\
7\end{array}$ & $\begin{array}{c}0.100 \\
00\end{array}$ & $\begin{array}{c}0.1519 \\
4\end{array}$ & $\begin{array}{c}0.002 \\
83\end{array}$ & 1272 & $\begin{array}{c}12 \\
1\end{array}$ & 1024 & 37 & 912 & $\begin{array}{l}1 \\
6\end{array}$ & $12.28 \%$ \\
\hline$R O 2 \# 67$ & 319 & 255 & $\begin{array}{c}1.2 \\
5\end{array}$ & 0.07246 & $\begin{array}{c}0.010 \\
79\end{array}$ & $\begin{array}{c}0.9883 \\
9\end{array}$ & $\begin{array}{c}0.143 \\
79\end{array}$ & $\begin{array}{c}0.0989 \\
3\end{array}$ & $\begin{array}{c}0.003 \\
16\end{array}$ & 999 & $\begin{array}{c}32 \\
3\end{array}$ & 698 & 73 & 608 & $\begin{array}{l}1 \\
9\end{array}$ & $14.80 \%$ \\
\hline RO2\#68 & 134 & 496 & $\begin{array}{c}0.2 \\
7\end{array}$ & 0.14539 & $\begin{array}{c}0.003 \\
85\end{array}$ & $\begin{array}{c}5.8637 \\
2\end{array}$ & $\begin{array}{c}0.130 \\
26\end{array}$ & $\begin{array}{c}0.2925 \\
1\end{array}$ & $\begin{array}{c}0.004 \\
22\end{array}$ & 2292 & 47 & 1956 & 19 & 1654 & $\begin{array}{l}2 \\
1\end{array}$ & $38.57 \%$ \\
\hline RO2\#69 & 395 & 544 & $\begin{array}{c}0.7 \\
3\end{array}$ & 0.06925 & $\begin{array}{c}0.004 \\
09\end{array}$ & $\begin{array}{c}0.9818 \\
8\end{array}$ & $\begin{array}{c}0.055 \\
87\end{array}$ & $\begin{array}{c}0.1028 \\
3\end{array}$ & $\begin{array}{c}0.001 \\
64\end{array}$ & 906 & $\begin{array}{c}12 \\
5\end{array}$ & 695 & 29 & 631 & $\begin{array}{l}1 \\
0\end{array}$ & $10.14 \%$ \\
\hline
\end{tabular}




\begin{tabular}{|c|c|c|c|c|c|c|c|c|c|c|c|c|c|c|c|c|}
\hline RO2\#70 & 164 & 453 & $\begin{array}{c}0.3 \\
6\end{array}$ & 0.08950 & $\begin{array}{c}0.006 \\
62\end{array}$ & $\begin{array}{c}1.0100 \\
3\end{array}$ & $\begin{array}{c}0.069 \\
63\end{array}$ & $\begin{array}{c}0.0817 \\
9\end{array}$ & $\begin{array}{c}0.002 \\
60\end{array}$ & 1415 & 84 & 709 & 35 & 507 & $\begin{array}{l}1 \\
5\end{array}$ & $39.84 \%$ \\
\hline RO2\#71 & 58 & 74 & $\begin{array}{c}0.7 \\
9\end{array}$ & 0.13109 & $\begin{array}{c}0.004 \\
26\end{array}$ & $\begin{array}{c}6.5056 \\
4\end{array}$ & $\begin{array}{c}0.202 \\
08\end{array}$ & $\begin{array}{c}0.3596 \\
3\end{array}$ & $\begin{array}{c}0.007 \\
35\end{array}$ & 2113 & 28 & 2047 & 27 & 1980 & $\begin{array}{l}3 \\
5\end{array}$ & $6.72 \%$ \\
\hline RO2\#72 & 47 & 42 & $\begin{array}{c}1.1 \\
1\end{array}$ & 0.07479 & $\begin{array}{c}0.009 \\
37\end{array}$ & $\begin{array}{c}0.9292 \\
6\end{array}$ & $\begin{array}{c}0.110 \\
39\end{array}$ & $\begin{array}{c}0.0900 \\
4\end{array}$ & $\begin{array}{c}0.004 \\
21\end{array}$ & 1063 & $\begin{array}{c}16 \\
5\end{array}$ & 667 & 58 & 556 & 2 & $19.96 \%$ \\
\hline RO2\#73 & 566 & 683 & $\begin{array}{c}0.8 \\
3\end{array}$ & 0.13483 & $\begin{array}{c}0.001 \\
60\end{array}$ & $\begin{array}{c}6.7193 \\
1\end{array}$ & $\begin{array}{c}0.075 \\
72\end{array}$ & $\begin{array}{c}0.3611 \\
4\end{array}$ & $\begin{array}{c}0.003 \\
96\end{array}$ & 2162 & & & 10 & 1988 & $\begin{array}{l}1 \\
9\end{array}$ & $8.75 \%$ \\
\hline RO2\#74 & 76 & 138 & $\begin{array}{c}0.5 \\
5\end{array}$ & 0.12919 & $\begin{array}{c}0.003 \\
07\end{array}$ & $\begin{array}{c}6.5868 \\
4\end{array}$ & $\begin{array}{c}0.149 \\
95\end{array}$ & $\begin{array}{c}0.3694 \\
9\end{array}$ & $\begin{array}{c}0.005 \\
94\end{array}$ & 08 & & 205 & 20 & 2027 & $\begin{array}{l}2 \\
8\end{array}$ & $2.96 \%$ \\
\hline RO2\#75 & 315 & 343 & $\begin{array}{c}0.9 \\
2\end{array}$ & 0.07486 & $\begin{array}{c}0.007 \\
78\end{array}$ & $\begin{array}{c}0.8121 \\
7\end{array}$ & $\begin{array}{c}0.082 \\
18\end{array}$ & $\begin{array}{c}0.0786 \\
8\end{array}$ & $\begin{array}{c}0.001 \\
85\end{array}$ & 1065 & $\begin{array}{c}21 \\
8\end{array}$ & 604 & 46 & 488 & $\begin{array}{l}1 \\
1\end{array}$ & $23.77 \%$ \\
\hline RO2\#76 & 36 & 70 & $\begin{array}{c}0.5 \\
2\end{array}$ & 0.06998 & $\begin{array}{c}0.007 \\
02\end{array}$ & $\begin{array}{c}1.0724 \\
3\end{array}$ & $\begin{array}{c}0.102 \\
08\end{array}$ & $\begin{array}{c}0.1110 \\
5\end{array}$ & $\begin{array}{c}0.004 \\
31\end{array}$ & 928 & $\begin{array}{c}13 \\
2\end{array}$ & 740 & 50 & 679 & 2 & $8.98 \%$ \\
\hline RO2\#77 & 30 & 46 & $\begin{array}{c}0.6 \\
5\end{array}$ & 0.12743 & $\begin{array}{c}0.005 \\
74\end{array}$ & $\begin{array}{c}6.4356 \\
3\end{array}$ & $\begin{array}{c}0.277 \\
55\end{array}$ & $\begin{array}{c}0.3659 \\
6\end{array}$ & $\begin{array}{c}0.009 \\
82\end{array}$ & 2063 & 40 & 2037 & 38 & 2010 & $\begin{array}{l}4 \\
6\end{array}$ & $2.64 \%$ \\
\hline RO2\#78 & 158 & 192 & $\begin{array}{c}0.8 \\
2\end{array}$ & 0.05913 & $\begin{array}{c}0.003 \\
22\end{array}$ & $\begin{array}{c}0.7910 \\
5\end{array}$ & $\begin{array}{c}0.041 \\
23\end{array}$ & $\begin{array}{c}0.0969 \\
4\end{array}$ & $\begin{array}{c}0.002 \\
00\end{array}$ & 572 & 77 & 592 & 23 & 596 & $\begin{array}{l}1 \\
2\end{array}$ & $-0.67 \%$ \\
\hline RO2\#79 & 71 & 89 & $\begin{array}{c}0.8 \\
0\end{array}$ & 0.11726 & $\begin{array}{c}0.004 \\
55\end{array}$ & $\begin{array}{c}5.1567 \\
8\end{array}$ & $\begin{array}{c}0.190 \\
58\end{array}$ & $\begin{array}{c}0.3186 \\
7\end{array}$ & $\begin{array}{c}0.007 \\
17\end{array}$ & 1915 & 36 & 1846 & 31 & 1783 & $\begin{array}{l}3 \\
5\end{array}$ & $7.40 \%$ \\
\hline RO2\#80 & 212 & 255 & $\begin{array}{c}0.8 \\
3\end{array}$ & 0.06321 & $\begin{array}{c}0.007 \\
36\end{array}$ & $\begin{array}{c}0.8198 \\
9\end{array}$ & $\begin{array}{c}0.093 \\
22\end{array}$ & $\begin{array}{c}0.0940 \\
7\end{array}$ & $\begin{array}{c}0.002 \\
32\end{array}$ & 715 & $\begin{array}{c}25 \\
9\end{array}$ & 608 & 52 & 580 & $\begin{array}{l}1 \\
4\end{array}$ & $4.83 \%$ \\
\hline RO2\#81 & 77 & 112 & $\begin{array}{c}0.6 \\
9\end{array}$ & 0.06087 & $\begin{array}{c}0.004 \\
15\end{array}$ & $\begin{array}{c}0.8656 \\
2\end{array}$ & $\begin{array}{c}0.056 \\
30\end{array}$ & $\begin{array}{c}0.1030 \\
4\end{array}$ & $\begin{array}{c}0.002 \\
63\end{array}$ & 635 & 96 & 633 & 31 & 632 & 5 & $0.16 \%$ \\
\hline RO2\#82 & 42 & 210 & $\begin{array}{c}0.2 \\
0\end{array}$ & 0.16716 & $\begin{array}{c}0.002 \\
74\end{array}$ & $\begin{array}{c}10.026 \\
31\end{array}$ & $\begin{array}{c}0.157 \\
42\end{array}$ & $\begin{array}{c}0.4346 \\
1\end{array}$ & $\begin{array}{c}0.005 \\
87\end{array}$ & 2529 & 12 & 2437 & 14 & 2326 & $\begin{array}{l}2 \\
6\end{array}$ & $8.73 \%$ \\
\hline RO2\#83 & 33 & 46 & $\begin{array}{c}0.7 \\
3\end{array}$ & 0.12124 & $\begin{array}{c}0.005 \\
46\end{array}$ & $\begin{array}{c}5.8001 \\
5\end{array}$ & $\begin{array}{c}0.249 \\
77\end{array}$ & $\begin{array}{c}0.3466 \\
4\end{array}$ & $\begin{array}{c}0.009 \\
02\end{array}$ & 1975 & 41 & 1946 & 37 & 1919 & $\begin{array}{l}4 \\
3\end{array}$ & $2.92 \%$ \\
\hline RO2\#84 & 56 & 76 & $\begin{array}{c}0.7 \\
4\end{array}$ & 0.14983 & $\begin{array}{c}0.008 \\
08\end{array}$ & $\begin{array}{c}7.1306 \\
5\end{array}$ & $\begin{array}{c}0.364 \\
55\end{array}$ & $\begin{array}{c}0.3448 \\
3\end{array}$ & $\begin{array}{c}0.011 \\
40\end{array}$ & 2344 & 45 & 2128 & 46 & 1910 & $\begin{array}{l}5 \\
5\end{array}$ & $22.72 \%$ \\
\hline RO2\#85 & 157 & 193 & $\begin{array}{c}0.8 \\
1\end{array}$ & 0.06892 & $\begin{array}{c}0.009 \\
71\end{array}$ & $\begin{array}{c}0.8063 \\
5\end{array}$ & $\begin{array}{c}0.110 \\
63\end{array}$ & $\begin{array}{c}0.0848 \\
5\end{array}$ & $\begin{array}{c}0.002 \\
71\end{array}$ & 896 & $\begin{array}{c}30 \\
9\end{array}$ & 600 & 62 & 525 & $\begin{array}{l}1 \\
6\end{array}$ & $14.29 \%$ \\
\hline RO2\#86 & 87 & 103 & $\begin{array}{c}0.8 \\
4\end{array}$ & 0.06789 & $\begin{array}{c}0.006 \\
17\end{array}$ & $\begin{array}{c}0.8681 \\
0\end{array}$ & $\begin{array}{c}0.074 \\
84\end{array}$ & $\begin{array}{c}0.0926 \\
4\end{array}$ & $\begin{array}{c}0.003 \\
18\end{array}$ & 865 & $\begin{array}{c}12 \\
2\end{array}$ & 635 & 41 & 571 & $\begin{array}{l}1 \\
9\end{array}$ & $11.21 \%$ \\
\hline RO2\#87 & 267 & 175 & 1.5 & 0.13716 & 0.002 & 7.5604 & 0.148 & 0.3993 & 0.005 & 2192 & 16 & 2180 & 18 & 2166 & 2 & $1.20 \%$ \\
\hline
\end{tabular}




\begin{tabular}{|c|c|c|c|c|c|c|c|c|c|c|c|c|c|c|c|c|}
\hline & & & 3 & & 80 & 7 & 34 & 8 & 91 & & & & & & 7 & \\
\hline RO2\#88 & 152 & 145 & $\begin{array}{c}1.0 \\
5\end{array}$ & 0.06106 & $\begin{array}{c}0.003 \\
88\end{array}$ & $\begin{array}{c}0.8520 \\
3\end{array}$ & $\begin{array}{c}0.051 \\
75\end{array}$ & $\begin{array}{c}0.1011 \\
0\end{array}$ & $\begin{array}{c}0.002 \\
41\end{array}$ & 641 & 90 & 626 & 28 & 621 & $\begin{array}{l}1 \\
4\end{array}$ & $0.81 \%$ \\
\hline RO2\#89 & 79 & 101 & $\begin{array}{c}0.7 \\
8\end{array}$ & 0.12046 & $\begin{array}{c}0.003 \\
62\end{array}$ & $\begin{array}{c}5.2227 \\
8\end{array}$ & $\begin{array}{c}0.149 \\
40\end{array}$ & $\begin{array}{c}0.3141 \\
3\end{array}$ & $\begin{array}{c}0.005 \\
77\end{array}$ & 1963 & 26 & 1856 & 24 & 1761 & $\begin{array}{l}2 \\
8\end{array}$ & $11.47 \%$ \\
\hline RO2\#90 & 119 & 186 & $\begin{array}{c}0.6 \\
4\end{array}$ & 0.04605 & $\begin{array}{c}0.006 \\
39\end{array}$ & $\begin{array}{c}0.4768 \\
0\end{array}$ & $\begin{array}{c}0.064 \\
87\end{array}$ & $\begin{array}{c}0.0751 \\
0\end{array}$ & $\begin{array}{c}0.002 \\
07\end{array}$ & & 25 & & 45 & 467 & $\begin{array}{l}1 \\
2\end{array}$ & $-15.20 \%$ \\
\hline RO2\#91 & 42 & 70 & $\begin{array}{c}0.6 \\
0\end{array}$ & 0.11801 & $\begin{array}{c}0.004 \\
73\end{array}$ & $\begin{array}{c}4.9153 \\
2\end{array}$ & $\begin{array}{c}0.187 \\
36\end{array}$ & $\begin{array}{c}0.3017 \\
7\end{array}$ & $\begin{array}{c}0.006 \\
92\end{array}$ & & & & 32 & 1700 & $\begin{array}{l}3 \\
4\end{array}$ & $13.29 \%$ \\
\hline RO2\#92 & 58 & 103 & $\begin{array}{c}0.5 \\
7\end{array}$ & 0.12783 & $\begin{array}{c}0.003 \\
59\end{array}$ & $\begin{array}{c}6.6089 \\
4\end{array}$ & $\begin{array}{c}0.178 \\
01\end{array}$ & $\begin{array}{c}0.3745 \\
5\end{array}$ & $\begin{array}{c}0.006 \\
80\end{array}$ & & 24 & 2061 & 24 & 2051 & $\begin{array}{l}3 \\
2\end{array}$ & $0.83 \%$ \\
\hline RO2\#93 & 118 & 96 & $\begin{array}{c}1.2 \\
3\end{array}$ & 0.18972 & $\begin{array}{c}0.012 \\
56\end{array}$ & $\begin{array}{c}2.3284 \\
5\end{array}$ & $\begin{array}{c}0.135 \\
13\end{array}$ & $\begin{array}{c}0.0889 \\
2\end{array}$ & $\begin{array}{c}0.003 \\
37\end{array}$ & 2740 & 49 & 1221 & 41 & 549 & $\begin{array}{l}2 \\
0\end{array}$ & $122.40 \%$ \\
\hline RO2\#94 & 85 & 73 & $\begin{array}{c}1.1 \\
6\end{array}$ & 0.10124 & $\begin{array}{c}0.007 \\
79\end{array}$ & $\begin{array}{c}1.4997 \\
3\end{array}$ & $\begin{array}{c}0.107 \\
23\end{array}$ & $\begin{array}{c}0.1073 \\
2\end{array}$ & $\begin{array}{c}0.003 \\
75\end{array}$ & 1647 & 81 & 930 & 44 & 657 & $\begin{array}{l}2 \\
2\end{array}$ & $41.55 \%$ \\
\hline RO2\#95 & 101 & 268 & $\begin{array}{c}0.3 \\
8\end{array}$ & 0.10816 & $\begin{array}{c}0.004 \\
83\end{array}$ & $\begin{array}{c}2.4730 \\
1\end{array}$ & $\begin{array}{c}0.100 \\
83\end{array}$ & $\begin{array}{c}0.1658 \\
2\end{array}$ & $\begin{array}{c}0.003 \\
01\end{array}$ & 1769 & 84 & 1264 & 29 & 989 & $\begin{array}{l}1 \\
7\end{array}$ & $27.81 \%$ \\
\hline NRO2\#01 & 109 & 252 & $\begin{array}{c}0.4 \\
3\end{array}$ & 0.06070 & $\begin{array}{c}0.001 \\
49\end{array}$ & $\begin{array}{c}0.7760 \\
0\end{array}$ & $\begin{array}{c}0.044 \\
05\end{array}$ & $\begin{array}{c}0.0926 \\
9\end{array}$ & $\begin{array}{c}0.001 \\
31\end{array}$ & 629 & 52 & 583 & 25 & 571 & 8 & $2.07 \%$ \\
\hline NRO2\#02 & 64 & 109 & $\begin{array}{c}0.5 \\
9\end{array}$ & 0.06360 & $\begin{array}{c}0.001 \\
70\end{array}$ & $\begin{array}{c}0.9606 \\
6\end{array}$ & $\begin{array}{c}0.065 \\
02\end{array}$ & $\begin{array}{c}0.1076 \\
8\end{array}$ & $\begin{array}{c}0.001 \\
65\end{array}$ & 729 & 56 & 684 & 34 & 659 & $\begin{array}{l}1 \\
0\end{array}$ & $3.69 \%$ \\
\hline NRO2\#03 & 322 & 261 & $\begin{array}{c}1.2 \\
3\end{array}$ & 0.0602 & $\begin{array}{c}0.001 \\
08\end{array}$ & $\begin{array}{c}0.8695 \\
9\end{array}$ & $\begin{array}{c}0.037 \\
69\end{array}$ & $\begin{array}{c}0.1030 \\
1\end{array}$ & $\begin{array}{c}0.001 \\
20\end{array}$ & 613 & 38 & 635 & 20 & 632 & 7 & $0.52 \%$ \\
\hline NRO2\#04 & 128 & 230 & $\begin{array}{c}0.5 \\
5\end{array}$ & 0.07168 & $\begin{array}{c}0.001 \\
07\end{array}$ & $\begin{array}{c}1.5126 \\
6\end{array}$ & $\begin{array}{c}0.067 \\
21\end{array}$ & $\begin{array}{c}0.1540 \\
2\end{array}$ & $\begin{array}{c}0.001 \\
71\end{array}$ & 977 & 30 & 936 & 27 & 924 & $\begin{array}{l}1 \\
0\end{array}$ & $1.30 \%$ \\
\hline NRO2\#05 & 362 & 451 & $\begin{array}{c}0.8 \\
0\end{array}$ & 0.09332 & $\begin{array}{c}0.001 \\
10\end{array}$ & $\begin{array}{c}1.2074 \\
7\end{array}$ & $\begin{array}{c}0.036 \\
79\end{array}$ & $\begin{array}{c}0.0926 \\
6\end{array}$ & $\begin{array}{c}0.000 \\
94\end{array}$ & 1494 & 22 & 804 & 17 & 571 & 6 & $40.76 \%$ \\
\hline NRO2\#06 & 156 & 453 & $\begin{array}{c}0.3 \\
4\end{array}$ & 0.06559 & $\begin{array}{c}0.000 \\
86\end{array}$ & $\begin{array}{c}0.8760 \\
2\end{array}$ & $\begin{array}{c}0.027 \\
09\end{array}$ & $\begin{array}{c}0.0954 \\
8\end{array}$ & $\begin{array}{c}0.000 \\
96\end{array}$ & 793 & 27 & 639 & 15 & 588 & 6 & $8.66 \%$ \\
\hline NRO2\#07 & 229 & 539 & $\begin{array}{c}0.4 \\
2\end{array}$ & 0.10193 & $\begin{array}{c}0.001 \\
24\end{array}$ & $\begin{array}{c}1.1979 \\
4\end{array}$ & $\begin{array}{c}0.037 \\
06\end{array}$ & $\begin{array}{c}0.0840 \\
1\end{array}$ & $\begin{array}{c}0.000 \\
87\end{array}$ & 1660 & 22 & 800 & 17 & 520 & 5 & $53.77 \%$ \\
\hline NRO2\#08 & 235 & 428 & $\begin{array}{c}0.5 \\
5\end{array}$ & 0.06165 & $\begin{array}{c}0.000 \\
92\end{array}$ & $\begin{array}{c}0.7973 \\
8\end{array}$ & $\begin{array}{c}0.027 \\
40\end{array}$ & $\begin{array}{c}0.0925 \\
4\end{array}$ & $\begin{array}{c}0.000 \\
98\end{array}$ & 662 & 32 & 595 & 15 & 571 & 6 & $4.36 \%$ \\
\hline NRO2\#09 & 167 & 314 & $\begin{array}{c}0.5 \\
3\end{array}$ & 0.10511 & $\begin{array}{c}0.001 \\
55\end{array}$ & $\begin{array}{c}1.4197 \\
6\end{array}$ & $\begin{array}{c}0.057 \\
77\end{array}$ & $\begin{array}{c}0.0942 \\
4\end{array}$ & $\begin{array}{c}0.001 \\
09\end{array}$ & 1716 & 27 & 897 & 24 & 581 & 6 & $54.55 \%$ \\
\hline
\end{tabular}




\begin{tabular}{|c|c|c|c|c|c|c|c|c|c|c|c|c|c|c|c|c|}
\hline NRO2\#10 & 201 & 34 & $\begin{array}{c}0.4 \\
6\end{array}$ & 0.09452 & $\begin{array}{c}0.001 \\
14\end{array}$ & $\begin{array}{c}1.2421 \\
6\end{array}$ & $\begin{array}{c}0.038 \\
95\end{array}$ & $\begin{array}{c}0.0933 \\
2\end{array}$ & $\begin{array}{c}0.000 \\
96\end{array}$ & 1519 & 23 & 820 & 18 & 575 & 6 & $42.57 \%$ \\
\hline NRO2\#11 & 103 & 194 & $\begin{array}{c}0.5 \\
3\end{array}$ & 0.06137 & $\begin{array}{c}0.001 \\
55\end{array}$ & $\begin{array}{c}0.8090 \\
4\end{array}$ & $\begin{array}{c}0.048 \\
15\end{array}$ & $\begin{array}{c}0.0949 \\
9\end{array}$ & $\begin{array}{c}0.001 \\
37\end{array}$ & 652 & 53 & 602 & 27 & 585 & 8 & $2.89 \%$ \\
\hline NRO2\#12 & 207 & 527 & $\begin{array}{c}0.3 \\
9\end{array}$ & 0.05901 & $\begin{array}{c}0.000 \\
80\end{array}$ & $\begin{array}{c}0.7699 \\
1\end{array}$ & $\begin{array}{c}0.023 \\
82\end{array}$ & $\begin{array}{c}0.0925 \\
2\end{array}$ & $\begin{array}{c}0.000 \\
93\end{array}$ & 568 & 29 & 580 & 14 & 570 & 6 & $1.63 \%$ \\
\hline NRO2\#13 & 185 & 257 & $\begin{array}{c}0.7 \\
2\end{array}$ & 0.06549 & $\begin{array}{c}0.001 \\
82\end{array}$ & $\begin{array}{c}0.8312 \\
6\end{array}$ & $\begin{array}{c}0.054 \\
69\end{array}$ & $\begin{array}{c}0.0931 \\
8\end{array}$ & $\begin{array}{c}0.001 \\
47\end{array}$ & 790 & & & 30 & 574 & 9 & $6.97 \%$ \\
\hline NRO2\#14 & 1019 & 656 & $\begin{array}{c}1.5 \\
5\end{array}$ & 0.14884 & $\begin{array}{c}0.001 \\
58\end{array}$ & $\begin{array}{c}1.6515 \\
2\end{array}$ & $\begin{array}{c}0.047 \\
33\end{array}$ & $\begin{array}{c}0.0785 \\
2\end{array}$ & $\begin{array}{c}0.000 \\
80\end{array}$ & & & & 18 & 487 & 5 & $103.18 \%$ \\
\hline NRO2\#15 & 1096 & 384 & $\begin{array}{c}2.8 \\
6\end{array}$ & 0.18774 & $\begin{array}{c}0.002 \\
30\end{array}$ & $\begin{array}{c}2.2183 \\
2\end{array}$ & $\begin{array}{c}0.081 \\
29\end{array}$ & $\begin{array}{c}0.0822 \\
5\end{array}$ & $\begin{array}{c}0.000 \\
93\end{array}$ & 2723 & 20 & 1187 & 26 & 510 & 6 & $132.93 \%$ \\
\hline NRO2\#16 & 154 & 383 & $\begin{array}{c}0.4 \\
0\end{array}$ & 0.06278 & $\begin{array}{c}0.001 \\
05\end{array}$ & $\begin{array}{c}0.8394 \\
8\end{array}$ & $\begin{array}{c}0.033 \\
30\end{array}$ & $\begin{array}{c}0.0951 \\
6\end{array}$ & $\begin{array}{c}0.001 \\
07\end{array}$ & 701 & 35 & 619 & 18 & 586 & 6 & $5.61 \%$ \\
\hline NRO2\#17 & 133 & 238 & $\begin{array}{c}0.5 \\
6\end{array}$ & 0.11750 & $\begin{array}{c}0.001 \\
13\end{array}$ & $\begin{array}{c}4.8771 \\
2\end{array}$ & $\begin{array}{c}0.208 \\
72\end{array}$ & $\begin{array}{c}0.3013 \\
4\end{array}$ & $\begin{array}{c}0.003 \\
01\end{array}$ & 1919 & 17 & 1798 & 36 & 1698 & $\begin{array}{l}1 \\
5\end{array}$ & $12.99 \%$ \\
\hline NRO2\#18 & 212 & 249 & $\begin{array}{c}0.8 \\
5\end{array}$ & 0.06454 & $\begin{array}{c}0.001 \\
14\end{array}$ & $\begin{array}{c}0.8759 \\
9\end{array}$ & $\begin{array}{c}0.037 \\
13\end{array}$ & $\begin{array}{c}0.1000 \\
5\end{array}$ & $\begin{array}{c}0.001 \\
17\end{array}$ & 760 & 37 & 639 & 20 & 615 & 7 & $3.92 \%$ \\
\hline NRO2\#19 & 93 & 228 & $\begin{array}{c}0.4 \\
1\end{array}$ & 0.17918 & $\begin{array}{c}0.001 \\
56\end{array}$ & 7.9964 & $\begin{array}{c}0.351 \\
34\end{array}$ & $\begin{array}{c}0.3123 \\
3\end{array}$ & $\begin{array}{c}0.003 \\
12\end{array}$ & 2645 & 14 & 2231 & 40 & 1752 & $\begin{array}{l}1 \\
5\end{array}$ & $50.97 \%$ \\
\hline NRO2\#20 & 147 & 281 & $\begin{array}{c}0.5 \\
3\end{array}$ & 0.06002 & $\begin{array}{c}0.001 \\
01\end{array}$ & $\begin{array}{c}0.8111 \\
8\end{array}$ & $\begin{array}{c}0.031 \\
98\end{array}$ & $\begin{array}{c}0.0964 \\
6\end{array}$ & $\begin{array}{c}0.001 \\
08\end{array}$ & 604 & 36 & 603 & 18 & 594 & 6 & $1.60 \%$ \\
\hline NRO2\#21 & 182 & 384 & $\begin{array}{c}0.4 \\
8\end{array}$ & 0.06343 & $\begin{array}{c}0.000 \\
99\end{array}$ & $\begin{array}{c}0.8332 \\
5\end{array}$ & $\begin{array}{c}0.030 \\
74\end{array}$ & $\begin{array}{c}0.0948 \\
6\end{array}$ & $\begin{array}{c}0.001 \\
03\end{array}$ & 723 & 33 & 615 & 17 & 584 & 6 & $5.34 \%$ \\
\hline NRO2\#22 & 210 & 268 & $\begin{array}{c}0.7 \\
8\end{array}$ & 007470 & $\begin{array}{c}0.001 \\
36\end{array}$ & $\begin{array}{c}1.1910 \\
7\end{array}$ & $\begin{array}{c}0.058 \\
61\end{array}$ & $\begin{array}{c}0.1169 \\
9\end{array}$ & $\begin{array}{c}0.001 \\
44\end{array}$ & 1061 & 36 & 797 & 27 & 713 & 8 & $11.68 \%$ \\
\hline $\mathrm{NRO} 2 \# 23$ & 81 & 167 & $\begin{array}{c}0.4 \\
8\end{array}$ & 0.06107 & $\begin{array}{c}0.001 \\
26\end{array}$ & $\begin{array}{c}0.8188 \\
1\end{array}$ & $\begin{array}{c}0.040 \\
09\end{array}$ & $\begin{array}{c}0.0983 \\
8\end{array}$ & $\begin{array}{c}0.001 \\
24\end{array}$ & 642 & 44 & 607 & 22 & 605 & 7 & $0.41 \%$ \\
\hline$N R O 2 \# 24$ & 456 & 920 & $\begin{array}{c}0.5 \\
0\end{array}$ & 0.11164 & $\begin{array}{c}0.001 \\
01\end{array}$ & $\begin{array}{c}1.4594 \\
9\end{array}$ & $\begin{array}{c}0.034 \\
63\end{array}$ & $\begin{array}{c}0.0942 \\
1\end{array}$ & $\begin{array}{c}0.000 \\
88\end{array}$ & 1826 & 16 & 914 & 14 & 580 & 5 & $57.44 \%$ \\
\hline NRO2\#25 & 85 & 214 & $\begin{array}{c}0.4 \\
0\end{array}$ & 0.13303 & $\begin{array}{c}0.001 \\
13\end{array}$ & $\begin{array}{c}7.3552 \\
3\end{array}$ & $\begin{array}{c}0.315 \\
52\end{array}$ & $\begin{array}{c}0.4018 \\
7\end{array}$ & $\begin{array}{c}0.003 \\
91\end{array}$ & 2138 & 15 & 2156 & 38 & 2178 & $\begin{array}{l}1 \\
8\end{array}$ & $-1.80 \%$ \\
\hline NRO2\#26 & 51 & 111 & $\begin{array}{c}0.4 \\
6\end{array}$ & 0.15972 & $\begin{array}{c}0.001 \\
69\end{array}$ & $\begin{array}{c}7.8794 \\
9\end{array}$ & $\begin{array}{c}0.452 \\
79\end{array}$ & $\begin{array}{c}0.3405 \\
8\end{array}$ & $\begin{array}{c}0.003 \\
76\end{array}$ & 2453 & 18 & 2217 & 52 & 1890 & $\begin{array}{l}1 \\
8\end{array}$ & $29.81 \%$ \\
\hline NRO2\#27 & 231 & 246 & 0.9 & 0.06319 & 0.001 & 0.8681 & 0.038 & 0.0989 & 0.001 & 715 & 39 & 635 & 21 & 608 & 7 & $4.31 \%$ \\
\hline
\end{tabular}




\begin{tabular}{|c|c|c|c|c|c|c|c|c|c|c|c|c|c|c|c|c|}
\hline & & & 4 & & 17 & 6 & 96 & 7 & 18 & & & & & & & \\
\hline NRO2\#28 & 490 & 292 & $\begin{array}{c}1.6 \\
7\end{array}$ & 0.09259 & $\begin{array}{c}0.001 \\
37\end{array}$ & $\begin{array}{c}1.1905 \\
9\end{array}$ & $\begin{array}{c}0.046 \\
00\end{array}$ & $\begin{array}{c}0.0886 \\
6\end{array}$ & $\begin{array}{c}0.001 \\
00\end{array}$ & 1480 & 28 & 796 & 21 & 548 & 6 & $45.40 \%$ \\
\hline NRO2\#29 & 188 & 197 & $\begin{array}{c}0.9 \\
5\end{array}$ & 0.06050 & $\begin{array}{c}0.001 \\
25\end{array}$ & $\begin{array}{c}0.8615 \\
7\end{array}$ & $\begin{array}{c}0.043 \\
15\end{array}$ & $\begin{array}{c}0.1013 \\
7\end{array}$ & $\begin{array}{c}0.001 \\
28\end{array}$ & 621 & 44 & 631 & 24 & 622 & 7 & $1.38 \%$ \\
\hline NRO2\#30 & 36 & 245 & $\begin{array}{c}0.1 \\
5\end{array}$ & 0.12327 & $\begin{array}{c}0.001 \\
06\end{array}$ & $\begin{array}{c}6.5247 \\
7\end{array}$ & $\begin{array}{c}0.272 \\
77\end{array}$ & $\begin{array}{c}0.3728 \\
9\end{array}$ & $\begin{array}{c}0.003 \\
60\end{array}$ & 2004 & 15 & & 37 & 2043 & $\begin{array}{l}1 \\
7\end{array}$ & $-1.90 \%$ \\
\hline NRO2\#31 & 60 & 82 & $\begin{array}{c}0.7 \\
3\end{array}$ & 0.11579 & $\begin{array}{c}0.001 \\
73\end{array}$ & $\begin{array}{c}4.2469 \\
0\end{array}$ & $\begin{array}{c}0.292 \\
61\end{array}$ & $\begin{array}{c}0.2510 \\
7\end{array}$ & $\begin{array}{c}0.003 \\
16\end{array}$ & 18 & & & 57 & 1444 & $\begin{array}{l}1 \\
6\end{array}$ & $31.04 \%$ \\
\hline NRO2\#32 & 134 & 144 & $\begin{array}{c}0.9 \\
4\end{array}$ & 0.08512 & $\begin{array}{c}0.001 \\
84\end{array}$ & $\begin{array}{c}1.0895 \\
3\end{array}$ & $\begin{array}{c}0.060 \\
19\end{array}$ & $\begin{array}{c}0.0910 \\
8\end{array}$ & $\begin{array}{c}0.001 \\
27\end{array}$ & 9 & 41 & 748 & 29 & 562 & 8 & $33.17 \%$ \\
\hline NRO2\#33 & 83 & 165 & $\begin{array}{c}0.5 \\
1\end{array}$ & 0.24753 & $\begin{array}{c}0.001 \\
81\end{array}$ & $\begin{array}{c}23.098 \\
34\end{array}$ & $\begin{array}{c}1.172 \\
63\end{array}$ & $\begin{array}{c}0.6427 \\
9\end{array}$ & 0.006 & 3169 & 12 & 3231 & 49 & 3200 & $\begin{array}{l}2 \\
5\end{array}$ & $-0.97 \%$ \\
\hline NRO2\#34 & 138 & 309 & $\begin{array}{c}0.4 \\
5\end{array}$ & 0.06397 & $\begin{array}{c}0.001 \\
10\end{array}$ & $\begin{array}{c}0.8331 \\
0\end{array}$ & $\begin{array}{c}0.033 \\
86\end{array}$ & $\begin{array}{c}0.0914 \\
4\end{array}$ & $\begin{array}{c}0.001 \\
04\end{array}$ & 741 & 36 & 615 & 19 & 564 & 6 & $9.10 \%$ \\
\hline NRO2\#35 & 138 & 299 & $\begin{array}{c}0.4 \\
6\end{array}$ & 0.05791 & $\begin{array}{c}0.000 \\
99\end{array}$ & $\begin{array}{c}0.7676 \\
5\end{array}$ & $\begin{array}{c}0.030 \\
29\end{array}$ & $\begin{array}{c}0.0960 \\
0\end{array}$ & $\begin{array}{c}0.001 \\
07\end{array}$ & 526 & 37 & 578 & 17 & 591 & 6 & $-2.13 \%$ \\
\hline NRO2\#36 & 171 & 230 & $\begin{array}{c}0.7 \\
4\end{array}$ & 0.15579 & $\begin{array}{c}0.001 \\
55\end{array}$ & $\begin{array}{c}4.7344 \\
7\end{array}$ & $\begin{array}{c}0.200 \\
42\end{array}$ & $\begin{array}{c}0.2191 \\
9\end{array}$ & $\begin{array}{c}0.002 \\
26\end{array}$ & 2411 & 17 & 1773 & 35 & 1278 & $\begin{array}{l}1 \\
2\end{array}$ & $88.67 \%$ \\
\hline NRO2\#37 & 171 & 458 & $\begin{array}{c}0.3 \\
7\end{array}$ & 0.06207 & $\begin{array}{c}0.000 \\
81\end{array}$ & $\begin{array}{c}0.8162 \\
2\end{array}$ & $\begin{array}{c}0.025 \\
17\end{array}$ & $\begin{array}{c}0.0925 \\
0\end{array}$ & $\begin{array}{c}0.000 \\
93\end{array}$ & 676 & 28 & 606 & 14 & 570 & 5 & $6.24 \%$ \\
\hline NRO2\#38 & 217 & 567 & $\begin{array}{c}0.3 \\
8\end{array}$ & 0.06106 & $\begin{array}{c}0.000 \\
83\end{array}$ & $\begin{array}{c}0.8462 \\
7\end{array}$ & $\begin{array}{c}0.027 \\
37\end{array}$ & $\begin{array}{c}0.0977 \\
8\end{array}$ & $\begin{array}{c}0.000 \\
99\end{array}$ & 642 & 29 & 623 & 15 & 601 & 6 & $3.53 \%$ \\
\hline NRO2\#39 & 187 & 290 & $\begin{array}{c}0.6 \\
5\end{array}$ & 0.0605 & $\begin{array}{c}0.001 \\
05\end{array}$ & $\begin{array}{c}0.7497 \\
3\end{array}$ & $\begin{array}{c}0.029 \\
62\end{array}$ & $\begin{array}{c}0.0931 \\
3\end{array}$ & $\begin{array}{c}0.001 \\
06\end{array}$ & 638 & 37 & 568 & 17 & 574 & 6 & $-1.03 \%$ \\
\hline NRO2\#40 & 48 & 90 & $\begin{array}{c}0.5 \\
3\end{array}$ & 0.07180 & $\begin{array}{c}0.001 \\
68\end{array}$ & $\begin{array}{c}1.2759 \\
3\end{array}$ & $\begin{array}{c}0.084 \\
35\end{array}$ & $\begin{array}{c}0.1204 \\
7\end{array}$ & $\begin{array}{c}0.001 \\
74\end{array}$ & 980 & 47 & 835 & 38 & 733 & $\begin{array}{l}1 \\
0\end{array}$ & $13.87 \%$ \\
\hline NRO2\#41 & 521 & 562 & $\begin{array}{c}0.9 \\
3\end{array}$ & 0.06260 & $\begin{array}{c}0.000 \\
91\end{array}$ & $\begin{array}{c}0.8591 \\
4\end{array}$ & $\begin{array}{c}0.030 \\
17\end{array}$ & $\begin{array}{c}0.0975 \\
8\end{array}$ & $\begin{array}{c}0.001 \\
02\end{array}$ & 695 & 31 & 630 & 16 & 600 & 6 & $4.92 \%$ \\
\hline NRO2\#42 & 57 & 712 & $\begin{array}{c}0.0 \\
8\end{array}$ & 0.11128 & $\begin{array}{c}0.001 \\
13\end{array}$ & $\begin{array}{c}1.4421 \\
0\end{array}$ & $\begin{array}{c}0.040 \\
07\end{array}$ & $\begin{array}{c}0.0903 \\
0\end{array}$ & $\begin{array}{c}0.000 \\
88\end{array}$ & 1820 & 18 & 907 & 17 & 557 & 5 & $62.68 \%$ \\
\hline NRO2\#43 & 202 & 518 & $\begin{array}{c}0.3 \\
9\end{array}$ & 0.05863 & $\begin{array}{c}0.000 \\
94\end{array}$ & $\begin{array}{c}0.7946 \\
0\end{array}$ & $\begin{array}{c}0.030 \\
00\end{array}$ & $\begin{array}{c}0.0946 \\
7\end{array}$ & $\begin{array}{c}0.001 \\
03\end{array}$ & 554 & 35 & 594 & 17 & 583 & 6 & $1.84 \%$ \\
\hline NRO2\#44 & 340 & 550 & $\begin{array}{c}0.6 \\
2\end{array}$ & 0.06899 & $\begin{array}{c}0.000 \\
89\end{array}$ & $\begin{array}{c}0.8874 \\
2\end{array}$ & $\begin{array}{c}0.027 \\
87\end{array}$ & $\begin{array}{c}0.0913 \\
1\end{array}$ & $\begin{array}{c}0.000 \\
92\end{array}$ & 898 & 26 & 645 & 15 & 563 & 5 & $14.50 \%$ \\
\hline
\end{tabular}




\begin{tabular}{|c|c|c|c|c|c|c|c|c|c|c|c|c|c|c|c|c|}
\hline NRO2\#45 & 83 & 26 & $\begin{array}{c}0.6 \\
6\end{array}$ & 0.11753 & $\begin{array}{c}0.001 \\
36\end{array}$ & $\begin{array}{c}5.6456 \\
0\end{array}$ & $\begin{array}{c}0.327 \\
39\end{array}$ & $\begin{array}{c}0.3493 \\
3\end{array}$ & $\begin{array}{c}0.003 \\
89\end{array}$ & 1919 & 21 & 1923 & 50 & 1931 & $\begin{array}{l}1 \\
9\end{array}$ & $-0.64 \%$ \\
\hline NRO2\#46 & 125 & 18 & $\begin{array}{c}0.3 \\
9\end{array}$ & 0.05941 & $\begin{array}{c}0.000 \\
99\end{array}$ & $\begin{array}{c}0.7536 \\
8\end{array}$ & $\begin{array}{c}0.029 \\
18\end{array}$ & $\begin{array}{c}0.0942 \\
8\end{array}$ & $\begin{array}{c}0.001 \\
05\end{array}$ & 582 & 36 & 570 & 17 & 581 & 6 & $-1.79 \%$ \\
\hline NRO2\#47 & 135 & 147 & $\begin{array}{c}0.9 \\
2\end{array}$ & 0.07129 & $\begin{array}{c}0.001 \\
20\end{array}$ & $\begin{array}{c}1.6601 \\
9\end{array}$ & $\begin{array}{c}0.088 \\
39\end{array}$ & $\begin{array}{c}0.1701 \\
2\end{array}$ & $\begin{array}{c}0.002 \\
03\end{array}$ & 966 & 34 & 993 & 34 & 1013 & 1 & $-4.64 \%$ \\
\hline NRO2\#48 & 38 & 50 & $\begin{array}{c}0.7 \\
6\end{array}$ & 0.05859 & $\begin{array}{c}0.002 \\
44\end{array}$ & $\begin{array}{c}0.8114 \\
7\end{array}$ & $\begin{array}{c}0.081 \\
10\end{array}$ & $\begin{array}{c}0.1001 \\
8\end{array}$ & $\begin{array}{c}0.002 \\
11\end{array}$ & 552 & & & 45 & 616 & 1 & $-1.98 \%$ \\
\hline NRO2\#49 & 123 & 0 & $\begin{array}{c}0.4 \\
2\end{array}$ & 0.06472 & $\begin{array}{l}0.001 \\
08\end{array}$ & $\begin{array}{c}0.9297 \\
9\end{array}$ & $\begin{array}{c}0.038 \\
88\end{array}$ & $\begin{array}{c}0.1038 \\
3\end{array}$ & $\begin{array}{c}0.001 \\
18\end{array}$ & & & & 20 & 637 & 7 & $4.82 \%$ \\
\hline NRO2\#50 & 70 & 70 & $\begin{array}{c}1.0 \\
0\end{array}$ & 0.06470 & $\begin{array}{c}0.002 \\
04\end{array}$ & $\begin{array}{c}0.9450 \\
3\end{array}$ & $\begin{array}{c}0.075 \\
28\end{array}$ & $\begin{array}{c}0.1031 \\
4\end{array}$ & $\begin{array}{c}0.001 \\
80\end{array}$ & 765 & 65 & 676 & 39 & 633 & 1 & $6.75 \%$ \\
\hline NRO2\#51 & 54 & 27 & $\begin{array}{c}0.4 \\
2\end{array}$ & 0.10238 & $\begin{array}{c}0.001 \\
45\end{array}$ & $\begin{array}{c}3.3808 \\
9\end{array}$ & $\begin{array}{c}0.196 \\
84\end{array}$ & $\begin{array}{c}0.2385 \\
9\end{array}$ & $\begin{array}{c}0.002 \\
82\end{array}$ & 1668 & 26 & 1500 & 46 & 1379 & 1 & $20.92 \%$ \\
\hline NRO2\#52 & 150 & 67 & $\begin{array}{c}0.5 \\
6\end{array}$ & 0.05910 & $\begin{array}{c}0.001 \\
11\end{array}$ & $\begin{array}{c}0.7497 \\
0\end{array}$ & $\begin{array}{c}0.032 \\
48\end{array}$ & $\begin{array}{c}0.0917 \\
4\end{array}$ & $\begin{array}{c}0.001 \\
08\end{array}$ & 571 & 40 & 568 & 19 & 566 & 6 & $0.39 \%$ \\
\hline NRO2\#53 & 100 & 78 & $\begin{array}{c}0.3 \\
6\end{array}$ & 0.17074 & $\begin{array}{c}0.001 \\
26\end{array}$ & $\begin{array}{c}11.064 \\
96\end{array}$ & $\begin{array}{c}0.446 \\
79\end{array}$ & $\begin{array}{c}0.4795 \\
1\end{array}$ & $\begin{array}{c}0.004 \\
52\end{array}$ & 2565 & 12 & 2529 & 38 & 2525 & $\begin{array}{l}2 \\
0\end{array}$ & $1.58 \%$ \\
\hline NRO2\#54 & 87 & 83 & $\begin{array}{c}0.2 \\
3\end{array}$ & 0.16503 & $\begin{array}{c}0.001 \\
24\end{array}$ & $\begin{array}{c}9.5664 \\
9\end{array}$ & $\begin{array}{c}0.370 \\
96\end{array}$ & $\begin{array}{c}0.3990 \\
2\end{array}$ & $\begin{array}{c}0.003 \\
73\end{array}$ & 2508 & 13 & 2394 & 36 & 2165 & $\begin{array}{l}1 \\
7\end{array}$ & $15.87 \%$ \\
\hline NRO2\#55 & 76 & 73 & $\begin{array}{c}1.0 \\
4\end{array}$ & 0.18740 & $\begin{array}{c}0.002 \\
05\end{array}$ & $\begin{array}{c}13.079 \\
10\end{array}$ & $\begin{array}{c}0.963 \\
66\end{array}$ & $\begin{array}{c}0.5319 \\
8\end{array}$ & $\begin{array}{c}0.006 \\
44\end{array}$ & 2720 & 18 & 2685 & 70 & 2750 & $\begin{array}{l}2 \\
7\end{array}$ & $-1.10 \%$ \\
\hline NRO2\#56 & 126 & 207 & $\begin{array}{c}0.6 \\
1\end{array}$ & 0.07294 & $\begin{array}{c}0.001 \\
09\end{array}$ & $\begin{array}{c}1.6885 \\
3\end{array}$ & $\begin{array}{c}0.080 \\
37\end{array}$ & $\begin{array}{c}0.1665 \\
6\end{array}$ & $\begin{array}{c}0.001 \\
86\end{array}$ & 1012 & 30 & 1004 & 30 & 993 & $\begin{array}{l}1 \\
0\end{array}$ & $1.12 \%$ \\
\hline NRO2\#57 & 62 & 199 & $\begin{array}{c}0.3 \\
1\end{array}$ & 0.06470 & $\begin{array}{c}0.001 \\
28\end{array}$ & $\begin{array}{c}0.8931 \\
6\end{array}$ & $\begin{array}{c}0.043 \\
44\end{array}$ & $\begin{array}{c}0.1006 \\
8\end{array}$ & $\begin{array}{c}0.001 \\
25\end{array}$ & 765 & 41 & 648 & 23 & 618 & 7 & $4.80 \%$ \\
\hline NRO2\#58 & 187 & 281 & $\begin{array}{c}0.6 \\
6\end{array}$ & 0.11460 & $\begin{array}{c}0.001 \\
65\end{array}$ & $\begin{array}{c}1.5601 \\
0\end{array}$ & $\begin{array}{c}0.064 \\
96\end{array}$ & $\begin{array}{c}0.0927 \\
2\end{array}$ & $\begin{array}{c}0.001 \\
07\end{array}$ & 1874 & 26 & 955 & 26 & 572 & 6 & $66.99 \%$ \\
\hline NRO2\#59 & 153 & 185 & $\begin{array}{c}0.8 \\
2\end{array}$ & 0.06553 & $\begin{array}{c}0.002 \\
42\end{array}$ & $\begin{array}{c}0.8080 \\
8\end{array}$ & $\begin{array}{c}0.070 \\
28\end{array}$ & $\begin{array}{c}0.0963 \\
7\end{array}$ & $\begin{array}{c}0.001 \\
92\end{array}$ & 791 & 76 & 601 & 39 & 593 & $\begin{array}{l}1 \\
1\end{array}$ & $1.40 \%$ \\
\hline NRO2\#60 & 30 & 205 & $\begin{array}{c}0.1 \\
4\end{array}$ & 0.12879 & $\begin{array}{c}0.001 \\
25\end{array}$ & $\begin{array}{c}5.9904 \\
1\end{array}$ & $\begin{array}{c}0.284 \\
65\end{array}$ & $\begin{array}{c}0.3299 \\
2\end{array}$ & $\begin{array}{c}0.003 \\
37\end{array}$ & 2082 & 17 & 1975 & 41 & 1838 & $\begin{array}{l}1 \\
6\end{array}$ & $13.25 \%$ \\
\hline NRO2\#61 & 507 & 634 & $\begin{array}{c}0.8 \\
0\end{array}$ & 0.11898 & $\begin{array}{c}0.001 \\
24\end{array}$ & $\begin{array}{c}1.4018 \\
0\end{array}$ & $\begin{array}{c}0.040 \\
61\end{array}$ & $\begin{array}{c}0.0846 \\
7\end{array}$ & $\begin{array}{c}0.000 \\
84\end{array}$ & 1941 & 19 & 890 & 17 & 524 & 5 & $69.79 \%$ \\
\hline NRO2\#62 & 305 & 486 & 0.6 & 0.07600 & 0.000 & 0.9456 & 0.031 & 0.0898 & 0.000 & 1095 & 26 & 676 & 16 & 554 & 5 & $21.92 \%$ \\
\hline
\end{tabular}




\begin{tabular}{|c|c|c|c|c|c|c|c|c|c|c|c|c|c|c|c|c|}
\hline & & & 3 & & 99 & 8 & 24 & 0 & 92 & & & & & & & \\
\hline NRO2\#63 & 61 & 97 & $\begin{array}{c}0.6 \\
3\end{array}$ & 0.06527 & $\begin{array}{c}0.001 \\
65\end{array}$ & $\begin{array}{c}1.1847 \\
5\end{array}$ & $\begin{array}{c}0.082 \\
94\end{array}$ & $\begin{array}{c}0.1242 \\
3\end{array}$ & $\begin{array}{c}0.001 \\
84\end{array}$ & 783 & 52 & 794 & 39 & 755 & $\begin{array}{l}1 \\
1\end{array}$ & $5.11 \%$ \\
\hline NRO2\#64 & 48 & 96 & $\begin{array}{c}0.5 \\
0\end{array}$ & 0.24565 & $\begin{array}{c}0.002 \\
23\end{array}$ & $\begin{array}{c}23.106 \\
10\end{array}$ & $\begin{array}{c}1.671 \\
36\end{array}$ & $\begin{array}{c}0.6375 \\
4\end{array}$ & $\begin{array}{c}0.007 \\
21\end{array}$ & 3157 & 14 & 3231 & 70 & 3179 & $\begin{array}{l}2 \\
8\end{array}$ & $-0.70 \%$ \\
\hline NRO2\#65 & 85 & 242 & $\begin{array}{c}0.3 \\
5\end{array}$ & 0.06025 & $\begin{array}{c}0.001 \\
15\end{array}$ & $\begin{array}{c}0.8219 \\
0\end{array}$ & $\begin{array}{c}0.038 \\
02\end{array}$ & $\begin{array}{c}0.1000 \\
7\end{array}$ & $\begin{array}{c}0.001 \\
20\end{array}$ & 612 & 41 & 60 & 21 & 615 & 7 & $-0.93 \%$ \\
\hline NRO2\#66 & 229 & 233 & $\begin{array}{c}0.9 \\
8\end{array}$ & 0.05851 & $\begin{array}{c}0.001 \\
15\end{array}$ & $\begin{array}{c}0.8561 \\
3\end{array}$ & $\begin{array}{c}0.041 \\
82\end{array}$ & $\begin{array}{c}0.0984 \\
7\end{array}$ & $\begin{array}{c}0.001 \\
20\end{array}$ & 549 & & & 23 & 605 & 7 & $3.73 \%$ \\
\hline NRO2\#67 & 56 & 131 & $\begin{array}{c}0.4 \\
3\end{array}$ & 0.13420 & $\begin{array}{c}0.001 \\
42\end{array}$ & $\begin{array}{c}7.3241 \\
3\end{array}$ & $\begin{array}{c}0.426 \\
51\end{array}$ & $\begin{array}{c}0.3925 \\
7\end{array}$ & $\begin{array}{c}0.004 \\
28\end{array}$ & 2154 & 18 & 2152 & 52 & 2135 & $\begin{array}{l}2 \\
0\end{array}$ & $0.89 \%$ \\
\hline NRO2\#68 & 156 & 280 & $\begin{array}{c}0.5 \\
6\end{array}$ & 0.07992 & $\begin{array}{c}0.001 \\
61\end{array}$ & $\begin{array}{c}1.1035 \\
8\end{array}$ & $\begin{array}{c}0.059 \\
12\end{array}$ & $\begin{array}{c}0.0988 \\
6\end{array}$ & $\begin{array}{c}0.001 \\
31\end{array}$ & 1195 & 39 & 755 & 29 & 608 & 8 & $24.26 \%$ \\
\hline NRO2\#69 & 122 & 50 & $\begin{array}{c}2.4 \\
5\end{array}$ & 0.11634 & $\begin{array}{c}0.002 \\
02\end{array}$ & $\begin{array}{c}5.4042 \\
0\end{array}$ & $\begin{array}{c}0.485 \\
35\end{array}$ & $\begin{array}{c}0.3541 \\
7\end{array}$ & $\begin{array}{c}0.005 \\
16\end{array}$ & 1901 & 31 & 1886 & 77 & 1955 & $\begin{array}{l}2 \\
5\end{array}$ & $-2.75 \%$ \\
\hline NRO2\#70 & 156 & 254 & $\begin{array}{c}0.6 \\
1\end{array}$ & 0.09942 & $\begin{array}{c}0.001 \\
54\end{array}$ & $\begin{array}{c}1.4874 \\
1\end{array}$ & $\begin{array}{c}0.067 \\
77\end{array}$ & $\begin{array}{c}0.1110 \\
3\end{array}$ & $\begin{array}{c}0.001 \\
32\end{array}$ & 1613 & 29 & 925 & 28 & 679 & 8 & $36.33 \%$ \\
\hline NRO2\#71 & 143 & 303 & $\begin{array}{c}0.4 \\
7\end{array}$ & 0.06126 & $\begin{array}{c}0.000 \\
98\end{array}$ & $\begin{array}{c}0.9276 \\
4\end{array}$ & $\begin{array}{c}0.037 \\
93\end{array}$ & $\begin{array}{c}0.1074 \\
2\end{array}$ & $\begin{array}{c}0.001 \\
18\end{array}$ & 648 & 34 & 666 & 20 & 658 & 7 & $1.32 \%$ \\
\hline NRO2\#72 & 105 & 168 & $\begin{array}{c}0.6 \\
2\end{array}$ & 0.06311 & $\begin{array}{c}0.001 \\
81\end{array}$ & $\begin{array}{c}0.7640 \\
1\end{array}$ & $\begin{array}{c}0.051 \\
39\end{array}$ & $\begin{array}{c}0.0885 \\
3\end{array}$ & $\begin{array}{c}0.001 \\
41\end{array}$ & 712 & 60 & 576 & 30 & 547 & 8 & $5.40 \%$ \\
\hline NRO2\#73 & 57 & 391 & $\begin{array}{c}0.1 \\
5\end{array}$ & 0.22101 & $\begin{array}{c}0.001 \\
78\end{array}$ & $\begin{array}{c}7.0343 \\
4\end{array}$ & $\begin{array}{l}0.257 \\
52\end{array}$ & $\begin{array}{c}0.2229 \\
7\end{array}$ & $\begin{array}{c}0.002 \\
15\end{array}$ & 2988 & 13 & 2116 & 33 & 1298 & $\begin{array}{l}1 \\
1\end{array}$ & $130.29 \%$ \\
\hline NRO2\#74 & 65 & 277 & $\begin{array}{c}0.2 \\
3\end{array}$ & 0.17099 & $\begin{array}{c}0.001 \\
25\end{array}$ & $\begin{array}{c}11.534 \\
58\end{array}$ & $\begin{array}{c}0.470 \\
70\end{array}$ & $\begin{array}{c}0.4912 \\
0\end{array}$ & $\begin{array}{c}0.004 \\
61\end{array}$ & 2567 & 12 & 2567 & 38 & 2576 & $\begin{array}{l}2 \\
0\end{array}$ & $-0.33 \%$ \\
\hline NRO2\#75 & 127 & 200 & $\begin{array}{c}0.6 \\
3\end{array}$ & 0.23415 & $\begin{array}{c}0.001 \\
70\end{array}$ & $\begin{array}{c}19.491 \\
91\end{array}$ & $\begin{array}{c}0.930 \\
56\end{array}$ & $\begin{array}{c}0.6227 \\
5\end{array}$ & $\begin{array}{c}0.006 \\
06\end{array}$ & 3081 & 12 & 3067 & 46 & 3121 & $\begin{array}{l}2 \\
4\end{array}$ & $-1.29 \%$ \\
\hline NRO2\#76 & 385 & 747 & $\begin{array}{c}0.5 \\
2\end{array}$ & 0.13996 & $\begin{array}{c}0.001 \\
38\end{array}$ & $\begin{array}{c}1.7190 \\
1\end{array}$ & $\begin{array}{c}0.050 \\
87\end{array}$ & $\begin{array}{c}0.0851 \\
3\end{array}$ & $\begin{array}{c}0.000 \\
84\end{array}$ & 2227 & 17 & 1016 & 19 & 527 & 5 & $92.84 \%$ \\
\hline NRO2\#77 & 352 & 571 & $\begin{array}{c}0.6 \\
2\end{array}$ & 0.10340 & $\begin{array}{c}0.001 \\
20\end{array}$ & $\begin{array}{c}1.3045 \\
2\end{array}$ & $\begin{array}{c}0.042 \\
58\end{array}$ & $\begin{array}{c}0.0922 \\
2\end{array}$ & $\begin{array}{c}0.000 \\
94\end{array}$ & 1686 & 21 & 848 & 19 & 569 & 6 & $49.09 \%$ \\
\hline NRO2\#78 & 22 & 51 & $\begin{array}{c}0.4 \\
3\end{array}$ & 0.16297 & $\begin{array}{c}0.002 \\
28\end{array}$ & $\begin{array}{c}10.163 \\
42\end{array}$ & $\begin{array}{c}0.929 \\
23\end{array}$ & $\begin{array}{c}0.4711 \\
5\end{array}$ & $\begin{array}{c}0.006 \\
54\end{array}$ & 2487 & 23 & 2450 & 85 & 2489 & $\begin{array}{l}2 \\
9\end{array}$ & $-0.08 \%$ \\
\hline NRO2\#79 & 147 & 292 & $\begin{array}{c}0.5 \\
0\end{array}$ & 0.06007 & $\begin{array}{c}0.001 \\
07\end{array}$ & $\begin{array}{c}0.8039 \\
9\end{array}$ & $\begin{array}{c}0.034 \\
77\end{array}$ & $\begin{array}{c}0.0973 \\
5\end{array}$ & $\begin{array}{c}0.001 \\
12\end{array}$ & 606 & 38 & 599 & 20 & 599 & 7 & $0.03 \%$ \\
\hline
\end{tabular}




\begin{tabular}{|c|c|c|c|c|c|c|c|c|c|c|c|c|c|c|c|c|}
\hline NRO2\#80 & 199 & 434 & $\begin{array}{c}0.4 \\
6\end{array}$ & 0.11356 & $\begin{array}{c}0.000 \\
99\end{array}$ & $\begin{array}{c}4.5884 \\
0\end{array}$ & $\begin{array}{c}0.174 \\
54\end{array}$ & $\begin{array}{c}0.2849 \\
2\end{array}$ & $\begin{array}{c}0.002 \\
71\end{array}$ & 1857 & 16 & 1747 & 32 & 1616 & $\begin{array}{l}1 \\
4\end{array}$ & $14.92 \%$ \\
\hline NRO2\#81 & 248 & 320 & $\begin{array}{c}0.7 \\
8\end{array}$ & 0.19167 & $\begin{array}{c}0.002 \\
23\end{array}$ & $\begin{array}{c}2.9062 \\
7\end{array}$ & $\begin{array}{c}0.118 \\
77\end{array}$ & $\begin{array}{c}0.1101 \\
6\end{array}$ & $\begin{array}{c}0.001 \\
23\end{array}$ & 2757 & 19 & 1384 & 31 & 674 & 7 & $105.36 \%$ \\
\hline NRO2\#82 & 59 & 199 & $\begin{array}{c}0.3 \\
0\end{array}$ & 0.06055 & $\begin{array}{c}0.001 \\
29\end{array}$ & $\begin{array}{c}0.8157 \\
5\end{array}$ & $\begin{array}{c}0.042 \\
66\end{array}$ & $\begin{array}{c}0.0963 \\
2\end{array}$ & $\begin{array}{c}0.001 \\
24\end{array}$ & 623 & 45 & 606 & 24 & 593 & 7 & $2.18 \%$ \\
\hline NRO2\#83 & 39 & 312 & $\begin{array}{c}0.1 \\
3\end{array}$ & 0.20049 & $\begin{array}{c}0.001 \\
38\end{array}$ & $\begin{array}{c}14.395 \\
56\end{array}$ & $\begin{array}{c}0.570 \\
25\end{array}$ & $\begin{array}{c}0.5367 \\
6\end{array}$ & $\begin{array}{c}0.004 \\
97\end{array}$ & 2830 & & 10 & 38 & 2770 & $\begin{array}{l}2 \\
1\end{array}$ & $2.18 \%$ \\
\hline NRO2\#84 & 82 & 164 & $\begin{array}{c}0.5 \\
0\end{array}$ & 0.06774 & $\begin{array}{c}0.001 \\
46\end{array}$ & $\begin{array}{c}1.0096 \\
1\end{array}$ & $\begin{array}{c}0.056 \\
96\end{array}$ & $\begin{array}{c}0.1089 \\
1\end{array}$ & $\begin{array}{c}0.001 \\
46\end{array}$ & & & 709 & 29 & 666 & 8 & $6.35 \%$ \\
\hline NRO2\#85 & 268 & 270 & $\begin{array}{c}0.9 \\
9\end{array}$ & 0.06373 & $\begin{array}{c}0.001 \\
11\end{array}$ & $\begin{array}{c}0.8868 \\
0\end{array}$ & $\begin{array}{c}0.039 \\
14\end{array}$ & $\begin{array}{c}0.1009 \\
0\end{array}$ & $\begin{array}{c}0.001 \\
17\end{array}$ & 3 & 36 & 645 & 21 & 620 & 7 & $4.03 \%$ \\
\hline NRO2\#86 & 162 & 257 & $\begin{array}{c}0.6 \\
3\end{array}$ & 0.12412 & $\begin{array}{c}0.001 \\
13\end{array}$ & $\begin{array}{c}5.7473 \\
6\end{array}$ & $\begin{array}{c}0.252 \\
00\end{array}$ & $\begin{array}{c}0.3434 \\
1\end{array}$ & $\begin{array}{c}0.003 \\
39\end{array}$ & 2016 & 16 & 1939 & 38 & 1903 & $\begin{array}{l}1 \\
6\end{array}$ & $5.95 \%$ \\
\hline NRO2\#87 & 120 & 421 & $\begin{array}{c}0.2 \\
8\end{array}$ & 0.19829 & $\begin{array}{c}0.001 \\
34\end{array}$ & $\begin{array}{c}10.931 \\
23\end{array}$ & $\begin{array}{c}0.377 \\
87\end{array}$ & $\begin{array}{c}0.3965 \\
8\end{array}$ & $\begin{array}{c}0.003 \\
58\end{array}$ & 2812 & 11 & 2517 & 32 & 2153 & $\begin{array}{l}1 \\
7\end{array}$ & $30.60 \%$ \\
\hline NRO2\#88 & 62 & 85 & $\begin{array}{c}0.7 \\
3\end{array}$ & 0.06421 & $\begin{array}{c}0.001 \\
61\end{array}$ & $\begin{array}{c}1.1160 \\
1\end{array}$ & $\begin{array}{c}0.076 \\
70\end{array}$ & $\begin{array}{c}0.1289 \\
2\end{array}$ & $\begin{array}{c}0.001 \\
90\end{array}$ & 749 & 52 & 761 & 37 & 782 & $\begin{array}{l}1 \\
1\end{array}$ & $-2.64 \%$ \\
\hline NRO2\#89 & 79 & 296 & $\begin{array}{c}0.2 \\
7\end{array}$ & 0.11911 & $\begin{array}{c}0.000 \\
98\end{array}$ & 6.0427 & $\begin{array}{l}0.240 \\
52\end{array}$ & $\begin{array}{c}0.3674 \\
1\end{array}$ & $\begin{array}{c}0.003 \\
47\end{array}$ & 1943 & 15 & 1982 & 35 & 2017 & $\begin{array}{l}1 \\
6\end{array}$ & $-3.68 \%$ \\
\hline NRO2\#90 & 174 & 416 & $\begin{array}{c}0.4 \\
2\end{array}$ & 0.06201 & $\begin{array}{c}0.000 \\
89\end{array}$ & $\begin{array}{c}0.9081 \\
9\end{array}$ & $\begin{array}{c}0.034 \\
29\end{array}$ & $\begin{array}{c}0.1068 \\
7\end{array}$ & $\begin{array}{c}0.001 \\
12\end{array}$ & 675 & 30 & 656 & 18 & 655 & 7 & $0.24 \%$ \\
\hline
\end{tabular}


Table 3. LA-MC-ICPMS zircon Lu-Hf isotopic data

\begin{tabular}{|c|c|c|c|c|c|c|c|c|c|c|c|c|}
\hline & \\
\hline Sample\#spot & $\begin{array}{c}\mathrm{t} \\
(\mathrm{Ma})\end{array}$ & ${ }^{176} \mathrm{Yb} /{ }^{177} \mathrm{Hf}$ & ${ }^{176} \mathrm{Lu} /{ }^{177} \mathrm{Hf}$ & ${ }^{176} \mathrm{Hf} /{ }^{177} \mathrm{Hf}$ & $2 \sigma$ & ${ }^{176} \mathrm{Hf} /{ }^{177} \mathrm{Hf}_{\mathrm{i}}$ & $\varepsilon_{\mathrm{Hf}}(\mathrm{t})$ & $2 \sigma$ & $\mathrm{T}_{\mathrm{DM} 1}$ & $2 \sigma$ & $\mathrm{T}_{\mathrm{DM}}^{2}$ & $2 \sigma$ \\
\hline \multicolumn{13}{|l|}{ 13FR12 } \\
\hline 13FR12\#02 & 525 & 0.058483 & 0.002305 & 0.282355 & 0.000017 & 0.282332 & -4.30 & 0.61 & 1311.6 & 24.97 & 1738.36 & 38.51 \\
\hline 13FR12\#03 & 629 & 0.021926 & 0.001011 & 0.282244 & 0.000020 & 0.282233 & -5.51 & 0.70 & 1421.5 & 27.47 & 1895.25 & 43.89 \\
\hline 13FR12\#09 & 620 & 0.017693 & 0.000783 & 0.282107 & 0.000014 & 0.282098 & 10.47 & .50 & 1602.9 & 19.60 & 2199.98 & 31.51 \\
\hline 13FR12\#11 & 1860 & 0.017425 & 0.000751 & 0.281487 & 0.000014 & 0.281460 & -4.89 & 0.51 & 2451.8 & 19.45 & 2812.20 & 31.29 \\
\hline 13FR12\#12 & 1873 & 0.013933 & 0.000547 & 0.281469 & 0.000015 & 0.281449 & -4.97 & 0.54 & 2463.0 & 20.37 & 2827.38 & 32.95 \\
\hline 13FR12\#13 & 1851 & 0.017211 & 0.000688 & 0.281315 & 0.000019 & 0.281291 & 11.10 & 0.68 & 2679.7 & 25.70 & 3186.53 & 41.41 \\
\hline 13FR12\#14 & 1866 & 0.017989 & 0.000725 & 0.281587 & 0.000016 & 0.281562 & -1.14 & 0.56 & 2312.9 & 21.61 & 2585.58 & 34.80 \\
\hline 13FR12\#15 & 1868 & 0.018340 & 0.000725 & 0.281457 & 0.000015 & 0.281432 & -5.72 & 0.55 & 2489.9 & 21.00 & 2869.26 & 33.81 \\
\hline 13FR12\#17 & 572 & 0.040271 & 0.001534 & 0.282324 & 0.000015 & 0.282308 & -4.13 & 0.54 & 1328.6 & 21.45 & 1763.97 & 33.80 \\
\hline 13FR12\#18 & 592 & 0.012470 & 0.0 & 0.282108 & 0.000015 & 0.282102 & $\begin{array}{c}- \\
10.95\end{array}$ & 0.53 & 1589.5 & 20.67 & 2208.72 & 33.51 \\
\hline 13FR12\#20 & 2659 & 0.012327 & 0.000485 & 0.281083 & 0.000012 & 0.281058 & -0.59 & 0.44 & 2976.9 & 16.40 & 3174.15 & 26.57 \\
\hline 13FR12\#21 & 610 & 0.016534 & 42 & 0.281809 & 0.000017 & 0.281801 & 21.23 & 0.59 & 2011.1 & 22.98 & 2864.76 & 36.98 \\
\hline 13FR12\#22 & 637 & 0.008194 & 0.000322 & 0.282154 & 0.000018 & 0.282150 & -8.25 & 0.65 & 1519.8 & 25.22 & 2073.63 & 41.04 \\
\hline 13FR12\#23 & 558 & 0.032691 & 0.001382 & 0.282041 & 0.000020 & 0.282027 & 14.39 & 0.70 & 1721.0 & 27.56 & 2397.83 & 43.60 \\
\hline 13FR12\#24 & 636 & 0.020878 & 0.000852 & 0.282154 & 0.000017 & 0.282144 & -8.50 & 0.58 & 1541.3 & 22.88 & 2088.72 & 36.72 \\
\hline 13FR12\#29 & 1895 & 0.018377 & 0.000714 & 0.281323 & 0.000014 & 0.281297 & -9.87 & 0.51 & 2671.3 & 19.58 & 3145.21 & 31.53 \\
\hline 13FR12\#33 & 567 & 0.020792 & 0.000858 & 0.281791 & 0.000016 & 0.281782 & 22.85 & 0.56 & 2041.8 & 21.90 & 2933.06 & 35.14 \\
\hline
\end{tabular}




\begin{tabular}{|c|c|c|c|c|c|c|c|c|c|c|c|c|}
\hline$¥ 37$ & 52 & 2 & 1 & 4 & 6 & 9 & 6.27 & 8 & 2 & 3 & 2 & 36.10 \\
\hline 3FR12\#38 & 1845 & 021268 & .000865 & 0.281458 & 0.000015 & 0.281428 & -6.37 & 0.52 & 2497.6 & 9.84 & 891.74 & 31.82 \\
\hline FR12\#43 & 31 & 5 & 00 & 9 & 4 & 1 & -7.46 & 0.51 & 2778.0 & 0 & 6 & .47 \\
\hline 3FR12\#44 & 1896 & 0.001300 & 0.000041 & 0.281430 & 0.000016 & 0.281429 & -5.17 & 0.58 & 24 & 21.80 & 57.38 & 5.74 \\
\hline FR12\#47 & 01 & 36 & .000762 & 1443 & 0.000017 & 11 & 1.27 & 0 & & 23.17 & 700.17 & 37.26 \\
\hline 13FR12\#50 & 2761 & 011582 & 0.000473 & 0.280998 & 0.000019 & 0.280973 & -1.23 & 0.6 & & 4.92 & 293.20 & 0.40 \\
\hline 13FR12\#53 & 2668 & 0.014857 & 0.000643 & 0.281005 & 0.000014 & 73 & -3 & & 1 & 18.24 & 3353.88 & 9.43 \\
\hline 13FR12\#55 & 555 & 0.034573 & 0.001320 & 0.2 & 0.000 & & & & 1792.6 & 28.77 & 2516.17 & 5.58 \\
\hline 13FR12\#59 & 364 & 0.013666 & 0.000551 & & 0.000014 & & -1 . & & 2742.4 & 18.88 & 2975.99 & 30.53 \\
\hline $3 \mathrm{~F}$ & 1635 & 0.0 & 0.000526 & 0 & 0 & 0 & 10.01 & 060 & 2 & 25 & 53.20 & 37.14 \\
\hline $13 \mathrm{FR}$ & 354 & 77 & 000540 & 7 & 0.000019 & 0.2 & -3.86 & 0 & 326 & 24.98 & 3525.85 & 0.42 \\
\hline 13FR12\#70 & 547 & 0.039044 & 0.001434 & 0.282351 & 0.000018 & 0.282336 & -3.67 & 0.62 & 1287.0 & 24.83 & 1715.96 & 39.22 \\
\hline 13FR12\#74 & 586 & 0.024285 & 0.000955 & 0281. & 00 & 0.281741 & $\begin{array}{c}- \\
23.86\end{array}$ & 0.69 & 2101.1 & 26.93 & 3010.46 & 43.09 \\
\hline 13FR12\#7 & 789 & .007174 & 0.000 & 0 & 0.000018 & 0.281060 & 2.53 & 0.66 & 2972.1 & 24.51 & 3087.14 & 39.91 \\
\hline 13 & 592 & 0.0 & 0.000674 & 0 & 0.000018 & 0.281935 & $\begin{array}{c}- \\
16.87\end{array}$ & 0. & 1824.7 & 24.48 & 2579.42 & 7 \\
\hline 13FR12\#78 & 584 & 0.021893 & & 0.282170 & 0.000018 & 0.282160 & -9.08 & 0.62 & 1520.6 & 24.49 & 2084.88 & 39.24 \\
\hline 13FR12\#79 & 669 & 0.01528 & 0.000731 & 0.281032 & 0.000017 & 0.280994 & -2.63 & 0.6 & 3064.9 & 23.16 & 3306.38 & 3728 \\
\hline 13 & 567 & 0.009473 & 0.000403 & 0.28 & 0.00001 & 0.282063 & $\begin{array}{c}- \\
12.89\end{array}$ & 0.60 & 1641 & 23.18 & 2311.24 & 37.64 \\
\hline 13 & 184 & 0.0 & 0.0 & 0. & 0.000 & 5 & 19.60 & 1 & 25 & 22.95 & 3702.80 & 36.98 \\
\hline $13 \mathrm{FR}$ & 510 & 0.023526 & 0.000959 & 0.28224 & 0.000016 & 0.282237 & -8.02 & 0.55 & 1417.2 & 21.70 & 1961.61 &.+ \\
\hline 13FR12\#94 & 593 & 0.015383 & 0.000743 & 0.282093 & 0.000017 & 0.282085 & - & 0.61 & 1621.2 & 23.60 & 2247.63 & 37.9 \\
\hline
\end{tabular}




\begin{tabular}{|c|c|c|c|c|c|c|c|c|c|c|c|c|}
\hline & & & & & & & 11.56 & & & & & \\
\hline 13FR12\#95 & 464 & 0.024426 & 0.000927 & 0.281976 & 0.000017 & 0.281968 & $\begin{array}{c}- \\
18.58\end{array}$ & 0.61 & 1790.9 & 23.88 & 2588.96 & 38.25 \\
\hline 13FR12\#96 & 608 & 0.022748 & 0.000891 & 0.282062 & 0.000015 & 0.282052 & 12.38 & 0.55 & 1670.1 & 21.38 & 2310.51 & 34.27 \\
\hline \multicolumn{13}{|l|}{ 13FR13 } \\
\hline 13FR13\#3 & 490 & 0.048002 & 0.001922 & 0.282358 & 0.000016 & 0.282340 & -4.82 & 0.58 & 1294.5 & 23.42 & 1744.09 & 36.51 \\
\hline 13FR13\#6 & 510 & 0.024506 & 0.000947 & 0.282527 & 0.000017 & 0.282518 & 1.92 & 0.62 & 1024.2 & 24.54 & 1333.02 & 39.28 \\
\hline 13FR13\#10 & 488 & 0.040156 & 0.001608 & 0.282307 & 0.000016 & 0.282292 & -6.57 & 0.57 & 1356.2 & 22.96 & 1853.06 & 36.10 \\
\hline 13FR13\#11 & 487 & 0.042767 & 0.001698 & 0.282413 & 0.000017 & 0.282398 & -2.84 & 0.59 & 1207.3 & 23.58 & 1616.73 & 36.99 \\
\hline 13FR13\#14 & 473 & 0.056597 & 0.002214 & 0.282402 & 0.000016 & 0.28238 & -3.72 & 0.55 & 1241.4 & 22.56 & 1661.22 & 34.89 \\
\hline 13FR13\#20 & 474 & 0.023911 & 0.000965 & 0.282141 & 0.000016 & 0.282133 & 12.52 & 0.55 & 1563.5 & 21.77 & 2217.01 & 34.82 \\
\hline 13FR13\#24 & 488 & 0.043704 & 0.001699 & 0.282475 & 0.000017 & 0.282460 & -0.64 & 0.62 & 1119.1 & 24.97 & 1477.91 & 39.16 \\
\hline 13FR13\#27 & 514 & 0.052257 & 0.002058 & 0.282324 & 0.000015 & 0.282304 & -5.56 & 0.52 & 1347.9 & 21.23 & 1809.12 & 32.97 \\
\hline 13FR13\#33 & 503 & 0.060344 & 0.002393 & 0.282353 & 0.000015 & 0.282330 & -4.88 & 0.55 & 1318.5 & 22.43 & 1757.87 & 34.51 \\
\hline 13FR13\#41 & 521 & 0.076177 & 0.002906 & 0.282253 & 0.000017 & 0.282225 & -8.21 & 0.60 & 1483.7 & 24.97 & 1981.19 & 37.87 \\
\hline 13FR13\#43 & 526 & 0.034296 & 0.001333 & 0.282394 & 0.000018 & 0.282381 & -2.56 & 0.62 & 1222.5 & 24.92 & 1629.25 & 39.47 \\
\hline 13FR13\#46 & 503 & 0.037833 & 0.001573 & 0.282286 & 0.000015 & 0.282271 & -6.96 & 0.53 & 1383.6 & 21.17 & 1888.95 & 33.33 \\
\hline 13FR13\#48 & 500 & 0.036132 & 0.001446 & 0.282248 & 0.000015 & 0.282235 & -8.32 & 0.54 & 1432.5 & 21.67 & 1972.68 & 34.22 \\
\hline 13FR13\#53 & 485 & 0.038727 & 0.001535 & 0.282293 & 0.000015 & 0.282279 & -7.09 & 0.52 & 1372.5 & 20.68 & 1883.15 & 32.58 \\
\hline 13FR13\#54 & 487 & 0.045178 & 0.001740 & 0.282396 & 0.000017 & 0.282380 & -3.46 & 0.59 & 1233.2 & 23.83 & 1656.06 & 37.34 \\
\hline 13FR13\#58 & 478 & 0.039401 & 0.001562 & 0.282335 & 0.000017 & 0.282321 & -5.77 & 0.59 & 1314.4 & 23.65 & 1794.67 & 37.23 \\
\hline 13FR13\#61 & 528 & 0.030247 & 0.001274 & 0.282134 & 0.000016 & 0.282122 & 11.70 & 0.56 & 1586.0 & 22.21 & 2206.64 & 35.24 \\
\hline
\end{tabular}




\begin{tabular}{|c|c|c|c|c|c|c|c|c|c|c|c|c|}
\hline 13FR13\#63 & 504 & .029805 & 0.001142 & 0.282545 & 0.000016 & 0.282534 & 2.37 & 0.56 & 1004.0 & 22.18 & 1300.11 & 35.31 \\
\hline 13FR13\#66 & 477 & 025408 & 001437 & 0.282304 & .000015 & 0.282291 & -6.85 & 0.53 & 1353.6 & 21.06 & 1862.22 & 3238 \\
\hline 13FR13\#68 & 514 & .021178 & 0.000899 & 0.282216 & .000015 & 0.282207 & -8.99 & 0.52 & 1457.4 & 20.32 & 025.84 & 2.56 \\
\hline 13FR13\#69 & 500 & .023006 & 000951 & .282250 & .000013 & 0.282241 & -8.09 & 0.47 & 1411 & 8.31 & 1958.04 & 29.30 \\
\hline 13FR13\#70 & 481 & 0.052137 & 0.002090 & 0.282410 & 0.000015 & 0.282391 & -3.21 & 0.55 & 1225.0 & 22.33 & 1635.45 & 4.65 \\
\hline 13FR13\#71 & 479 & .023622 & 0.000921 & 0.282571 & 0.000017 & 82563 & 2.83 & 0.61 & 961.2 & 24.33 & 251.35 & 8.96 \\
\hline 13FR13\#73 & 478 & 0.037503 & 0.001475 & 0.282435 & 0.000015 & 0.282422 & -2.18 & 0.53 & 1168.9 & 21.11 & 1568.23 & 33.31 \\
\hline 13FR13\#76 & 487 & 0.052935 & 0.002092 & 0.282326 & 0.000015 & 0.282307 & -6.05 & 0.54 & 1345.6 & 21.98 & 1819.26 & 4.11 \\
\hline 13FR13\#77 & 468 & 0.012285 & 0.000537 & 0.282213 & 0.000019 & & & 0.69 & 1447.3 & 26.72 & 2052.65 & 43.23 \\
\hline 13FR13\#86 & 490 & 0.049110 & 0.001928 & 0.282327 & 0.000023 & 0.282310 & -5.90 & 0.81 & 1338.3 & 32.69 & 1812.19 & 50.95 \\
\hline 13FR13\#88 & 492 & 0068383 & 0.002670 & 0.282406 & 000017 & 0.282381 & -3.33 & 50 & 12511 & 24.30 & 651.14 & 7.11 \\
\hline \multicolumn{13}{|l|}{ 13FR18-1 } \\
\hline $12 \mathrm{CD} 10$ & 2427 & 047797 & 0.001659 & 81161 & & 0.281084 & -5.09 & 0.61 & 2961.7 & 23.57 & 3266.52 & 37.01 \\
\hline 13FR18-1\#04 & 2577 & 0.017211 & 0.000715 & & 0.000017 & 0.281097 & -1.14 & 0.59 & 2928.7 & 22.26 & 3143.49 & 35.85 \\
\hline $13 \mathrm{~F}$ & 623 & 0. & 0.000610 & 2276 & 0.0 & 0.28 & -4.37 & 0 & 1 & 9 & 1 & 22 \\
\hline 13FR18-1\#08 & 620 & 0.020319 & 0.000851 & 0.282173 & 0.000018 & 0.282163 & -8.19 & 0.65 & 1515.3 & 25.32 & 2056.67 & 40.63 \\
\hline 13FR18-1\#10 & 2579 & 0.009882 & 0.000397 & 0.280977 & 0.000016 & 0.280958 & -0.04 & 0.50 & ग11.1 & 20.81 & 3443.14 & 33.89 \\
\hline 13FR18-1\#12 & 2579 & 0.017287 & 0.000686 & 0.281005 & 0.000016 & 0.280971 & -5.56 & 0.57 & 3096.9 & 21.38 & 3413.73 & 34.46 \\
\hline 13FR18-1\#13 & 1949 & 0.014748 & 0.000620 & 0.281336 & 0.000017 & 0.281313 & -8.05 & 0.61 & 2646.8 & 23.00 & 3075.61 & 37.14 \\
\hline 13FR18-1\#21 & 2160 & 0.026575 & 0.001075 & 0.281495 & 0.000024 & 0.281451 & 1.72 & 0.87 & 2461.3 & 33.42 & 2640.54 & 53.31 \\
\hline$-1 \# 25$ & 627 & 0.03 & 0.001300 & 891 & 0.000017 & $0.2818^{\circ}$ & $\begin{array}{c}- \\
18.19\end{array}$ & 0.61 & 1926.8 & 23.98 & 2687.89 & 8.02 \\
\hline 13FR18-1\#26 & 620 & 0.027157 & 0.001117 & 0.281831 & 0.000020 & 0.281818 & 20.40 & 0.70 & 2001.1 & 27.29 & 820.47 & 18 \\
\hline
\end{tabular}




\begin{tabular}{|c|c|c|c|c|c|c|c|c|c|c|c|c|}
\hline 13FR18-1\#27 & 626 & 0.014099 & 0.000573 & 0.282061 & 0.000015 & 0.282054 & 11.90 & 0.53 & 1658.1 & 20.53 & 2294.45 & 33.19 \\
\hline 3FR18-1\#30 & 2928 & 0.055861 & 0.002095 & 0.280938 & 0.000022 & 0.280820 & -2.74 & 0.79 & 3307.0 & 30.73 & 3515.96 & 47.68 \\
\hline 3FR18-1\#37 & 624 & 0.020492 & 0.000910 & 0.282028 & 0.000014 & 0.282017 & 13.24 & 0.50 & 1717.9 & 19 & 2376.63 & 31.55 \\
\hline 3FR18-1\#41 & 1939 & 0.014026 & 0.000587 & 0.281121 & 0.000015 & 0.281099 & $\begin{array}{c}- \\
15.89\end{array}$ & 0.54 & & 9 & 3547.57 & 33.11 \\
\hline 3FR18-1\#52 & 623 & 0.028488 & 0.001115 & 0.282028 & 0.000020 & 0.282015 & $\begin{array}{c}- \\
13.34\end{array}$ & & & 28.50 & 2381.97 & 45.42 \\
\hline 3FR18-1\#60 & 617 & 0.011639 & 0.000503 & 0.282090 & 0.000015 & 0.28 & 11.03 & 0.53 & 1614.8 & 0.68 & 233.00 & 49 \\
\hline 3FR18-1\#62 & 1951 & 0.013482 & 0.000568 & 0.281469 & 0.000016 & 0.28 & -3.22 & 0.56 & 2464.0 & 21.43 & 2780.42 & 34.65 \\
\hline 13FR18-1\#67 & 627 & 0.016313 & 0.000773 & 0.282099 & 0.000014 & 0.2 & 10.60 & 0.50 & 1613.8 & 19.65 & 2213.86 & 31.59 \\
\hline 13FR18-1\#69 & 619 & 0.026613 & 0.001125 & 0.282245 & 0.000019 & 0.282232 & -5.76 & 0.66 & 1425.2 & 26.06 & 1903.27 & 41.51 \\
\hline 3FR18-1\#76 & 1942 & 0.013132 & 0.000530 & 0.281406 & 0.00 & 0.281386 & -5.63 & 0.64 & 2547.2 & 24.23 & 2921.50 & 39.21 \\
\hline 13FR18-1\#77 & 2906 & 0.035235 & 0.001363 & 0.280958 & 0.000018 & 0.280882 & -1.05 & 0.65 & 3215.9 & 24.71 & 3396.52 & 39.11 \\
\hline 13FR18-1\#80 & 1930 & 0.011248 & 0.000458 & 0.2 & 0.000015 & 0.281500 & -1.84 & 0.53 & 2391.8 & 20.10 & 2678.87 & 32.59 \\
\hline 3FR18-1\#81 & 2652 & 0.017811 & 0.0 & 0.2 & 0.000016 & 0.280955 & -4.42 & 0.56 & 3117.5 & 21.17 & 3401.82 & 34.10 \\
\hline $1 \# 84$ & 623 & 0.031966 & 0.00 & 0.281932 & 0.000019 & 0.281916 & $\begin{array}{c}- \\
16.87\end{array}$ & 0.66 & 1873.8 & 26.05 & 2602.41 & 41.23 \\
\hline 13FR18-1\#86 & 1926 & 0.017259 & 0.000680 & 0.281394 & 0.000018 & 0.281369 & -6.61 & 0.64 & 2573.2 & 24.55 & 2969.19 & 39.57 \\
\hline 13FR18-1\#87 & 1923 & 0.015045 & 0.000596 & 0.281381 & 0.000019 & 0.281360 & -7.00 & 0.68 & 2584.1 & 25.73 & 2991.06 & 41.56 \\
\hline 13FR18-1\#88 & 617 & 0.023436 & 0.000915 & 0.282335 & 0.000018 & 0.282325 & -2.51 & 0.62 & 1291.3 & 24.57 & 1697.20 & 39.37 \\
\hline 13FR18-1\#97 & 1940 & 0.012570 & 0.000512 & 0.281286 & 0.000016 & 0.281267 & -9.91 & 0.57 & 2707.5 & 21.40 & 3182.72 & 34.66 \\
\hline \multicolumn{13}{|l|}{ 13FR19 } \\
\hline FR19\#01 & 1728 & 0.038841 & 0.001466 & 0.281455 & 0.000017 & .281407 & -9.82 & 0.60 & 2542.1 & 23.39 & 3012.90 & 36.93 \\
\hline
\end{tabular}




\begin{tabular}{|c|c|c|c|c|c|c|c|c|c|c|c|c|}
\hline 13FR19\#05 & 2057 & 0.003785 & 0.000134 & 0.281386 & 0.000014 & 0.281381 & -3.14 & 0.51 & 2547.2 & 19.20 & 858.57 & 31.40 \\
\hline 13FR19\#06 & 1882 & 0.017779 & 0.000665 & 0.281509 & 0.000018 & 0.281485 & -3.48 & 0.63 & 2415.6 & 23.97 & 2742.55 & 38.65 \\
\hline 13FR19\#07 & 1679 & 0.015611 & 0.000644 & 0.281503 & 0.000015 & 0.281482 & -8.26 & 0.54 & 2423.0 & 20.53 & 879.42 & 33.12 \\
\hline 13FR19\#08 & 567 & 0.042033 & 0.001591 & 0.282203 & 0.000016 & 0.282186 & -8.55 & 0.57 & 1502 & 22.73 & 2038.36 & 35.76 \\
\hline 3FR19\#09 & 2111 & 0.011361 & 0.000434 & 0.281403 & 0.000015 & 0.281385 & -1.74 & .55 & 25 & 20.66 & 814.64 & 33.52 \\
\hline 3FR19\#16 & 615 & 0.011173 & 0.000466 & 0.282216 & 0.000015 & 0.282211 & -6.58 & .54 & 139.8 & 21.13 & 1952.17 & 34.26 \\
\hline 13FR19\#18 & 646 & 0.017295 & 0.000592 & 0.282138 & 0.000026 & 0.282131 & -8.71 & 0.93 & 1552.1 & 36.11 & 109.94 & 58.34 \\
\hline 13FR19\#20 & 1915 & 0.021449 & 0.000827 & 0.281296 & 0.000032 & 266 & & .15 & 2714.9 & 43.81 & 3199.47 & 70.35 \\
\hline 3FR19\#22 & 587 & 0.024604 & 0.001003 & 0.282053 & 0.000028 & & 13. & 1.00 & 1688.0 & 39.18 & 2346.56 & 2.61 \\
\hline \#23 & 64 & 0.0 & 0 & 0.2 & 0.000 & & 20.90 & 77 & .8 & .41 & 85 & 1.84 \\
\hline 13FR19\#26 & 702 & 0.043480 & 0.001571 & 0.282070 & 0.000017 & 0.282049 & $\begin{array}{c}- \\
10.37\end{array}$ & 0.61 & 1689.7 & 24.09 & 2256.59 & 37.92 \\
\hline 13FR19\#27 & 623 & 0.026821 & 0.001099 & 0.282162 & 0000021 & 0. & -8.60 & 0.76 & 0.1 & 29.93 & 4.91 & 47.70 \\
\hline 13FR19\#28 & 1892 & 0.008432 & 0.000315 & & 0.000023 & 0.281640 & 2.24 & 0.81 & 2202.5 & 30.69 & 2397.39 & 49.94 \\
\hline 13FR19\#29 & 2191 & 0.016676 & 0.000698 & 0.281 & 0.000023 & 348 & -1.22 & 0.82 & 2596.9 & 31.22 & 2845.04 & 50.31 \\
\hline 13FR19\#30 & 575 & 0.02 & & 0.28 & 0.000022 & 0.282083 & 12.02 & 0.76 & 1636.9 & 30.08 & 2262.88 & 47.82 \\
\hline 13FR19\#37 & 2427 & 0.035105 & 0.001464 & 0.281369 & 0.000019 & 0.281302 & 2.63 & 0.68 & 2659.9 & 26.30 & 2794.59 & 41.51 \\
\hline 13FR19\#38 & 572 & 0.02 & 0.000962 & 0.282073 & 0.000024 & 0.282062 & $\begin{array}{c}- \\
12.81\end{array}$ & 0.84 & 1658.4 & 33.04 & 2310.16 & 52.86 \\
\hline 13FR19\#41 & 411 & 0.019623 & 0.000815 & 0.282565 & 0.000031 & 0.282558 & 1.14 & 1.10 & 968.0 & 43.56 & 1305.58 & 69.97 \\
\hline \multicolumn{13}{|l|}{ 13FR21 } \\
\hline 3FR21\#11 & 534 & 0.020750 & 0.000870 & 0.282555 & 0.000014 & 0.282546 & 3.45 & 0.49 & 983.3 & 19.42 & 1254.64 & 31.15 \\
\hline 13FR21\#13 & 499 & 0.050845 & 0.001993 & 0.282604 & 0.000016 & 0.282586 & 4.08 & 0.58 & 941.6 & 23.83 & 1187.68 & 37.08 \\
\hline
\end{tabular}




\begin{tabular}{|c|c|c|c|c|c|c|c|c|c|c|c|c|}
\hline 13FR21\#14 & 483 & 0.036855 & 0.001428 & 0.282657 & 0.000017 & 0.282644 & 5.77 & 0.60 & 852.9 & 24.00 & 1067.37 & 37.91 \\
\hline 3 FR21\#16 & 540 & 0.030997 & 0.001443 & 0.282610 & 0.000018 & 0.282595 & 5.34 & 0.63 & 919.6 & 25.45 & 1139.60 & 40.20 \\
\hline 13FR21\#25 & 533 & 0.003303 & 0.000171 & 0.282522 & 0.000014 & 0.282520 & 2.53 & 0.48 & 1010.3 & 18.73 & 1312.82 & 30.60 \\
\hline 13FR21\#26 & 544 & 0.008824 & 0.000377 & 0.282515 & 0.000015 & 0.282511 & 2.45 & 0.54 & 1025 & 21.21 & 1326.50 & 34.47 \\
\hline 13FR21\#28 & 545 & 0.027906 & 0.001293 & 0.282672 & 0.000017 & 0.282659 & 7.70 & 0.59 & 827.6 & 23.53 & 993.15 & 37.32 \\
\hline 13FR21\#30 & 491 & 0.050671 & 0.001938 & 0.282431 & 0.000016 & 0.282413 & -2.22 & ) & 3 & 22.68 & 1580.73 & 35.34 \\
\hline 13FR21\#31 & 482 & 0.033574 & 0.001306 & 0.282606 & 0.000016 & 0.282594 & 3.99 & 0.56 & 922.1 & 22.57 & 1179.68 & 35.79 \\
\hline 13FR21\#34 & 540 & 0.013126 & 0.000540 & 0.282527 & 0.000016 & 0.282522 & & 0.57 & 1012.5 & 22.26 & 1304.51 & 36.01 \\
\hline 13FR21\#38 & 482 & 0.031201 & 0.001258 & 0.282403 & 0.000015 & 0. & & 0.52 & 1207.3 & 20.51 & 1633.29 & 32.55 \\
\hline 13FR21\#39 & 504 & 0.046133 & 0.001821 & 0.282563 & 0.000013 & 0.282546 & 2.79 & 0.46 & 996.1 & 18.73 & 1273.26 & 29.28 \\
\hline 13FR21\#43 & 482 & 0.032090 & 0.001282 & 0.282522 & 0.000015 & 0.282510 & 1.03 & 0.52 & 1040.5 & 20.92 & 1367.87 & 33.19 \\
\hline 13FR21\#44 & 542 & 0.031041 & 0.001216 & 0.282703 & 0.000016 & 0.282690 & 8.74 & 0.56 & 782.7 & 22.55 & 924.54 & 35.84 \\
\hline 13FR21\#45 & 487 & 0.039174 & 0.001561 & 0.282559 & 0.000015 & 0.282545 & 2.38 & 0.55 & 994.7 & 22.12 & 1286.29 & 34.82 \\
\hline 13FR21\#46 & 551 & 0.042025 & 0.001790 & & 0.000016 & 0.282614 & 6.24 & 0.57 & 896.0 & 23.04 & 1090.77 & 36.05 \\
\hline 13FR21\#47 & 492 & 0.019574 & & 0.281953 & 0.000020 & 0.281946 & $\begin{array}{c}- \\
18.72\end{array}$ & 0.72 & 1815.4 & 27.92 & 2619.28 & 44.88 \\
\hline 13FR21\#48 & 543 & 0.009367 & & 0.282519 & 0.000016 & 0.282515 & 2.56 & 0.57 & 1020.2 & 22.16 & 1318.32 & 35.99 \\
\hline 13FR21\#49 & 483 & 0.036219 & 0.001421 & 0.282556 & 0.000017 & 0.282544 & 2.23 & 0.59 & 995.2 & 23.66 & 1292.46 & 37.38 \\
\hline 13FR21\#54 & 495 & 0.0669 & 0.002523 & 0.282380 & 0.000015 & 0.282356 & -4.14 & 0.51 & 1284.0 & 21.20 & 1704.64 & 32.50 \\
\hline 13FR21\#55 & 499 & 0.054656 & 0.002113 & 0.282436 & 0.000018 & 0.282417 & -1.91 & 0.64 & 1187.6 & 26.02 & 1566.78 & 40.35 \\
\hline 13FR21\#56 & 485 & 0.026082 & 0.000943 & 0.282281 & 0.000015 & 0.282272 & -7.32 & 0.53 & 1367.9 & 20.74 & 1898.36 & 33.19 \\
\hline 13FR21\#63 & 500 & 0.013121 & 0.000465 & 0.282254 & 0.000015 & 0.282250 & -7.78 & 0.55 & 1387.6 & 21.26 & 1938.95 & 34.46 \\
\hline 13FR21\#65 & 544 & 0.059450 & 0.002319 & 0.282214 & 0.000016 & 0.282191 & -8.90 & 0.58 & 1515.8 & 23.62 & 2042.51 & 36.42 \\
\hline
\end{tabular}




\begin{tabular}{|c|c|c|c|c|c|c|c|c|c|c|c|c|}
\hline 13FR21\#69 & 547 & 0.013950 & 0.000634 & 0.282718 & 0.000017 & 0.282711 & 9.60 & 0.59 & 749.6 & 23.35 & 873.99 & 37.69 \\
\hline 13FR21\#70 & 483 & .021603 & 0.000920 & 0.282523 & 0.000016 & 0.282515 & 1.20 & 0.55 & 1029.1 & 21.76 & 1357.76 & 34.85 \\
\hline 13FR21\#72 & 542 & 0.022195 & 0.000957 & 0.282316 & 0.000015 & 0.282306 & -4.86 & 54 & 1320.0 & 1.30 & 786.87 & 4.08 \\
\hline 13FR21\#74 & 502 & 0.027179 & 0.001120 & 0.282041 & 0.000014 & 0.282030 & $\begin{array}{c}- \\
15.51\end{array}$ & 0.51 & & 1 & 2425.79 & 31.72 \\
\hline 13FR21\#77 & 497 & 0.037966 & 0.001500 & 0.282576 & 0.000016 & 0.282562 & 3.18 & 0.56 & 970.0 & 22.46 & 1242.81 & 35.42 \\
\hline 13FR21\#78 & 542 & .016332 & 0.000692 & 0.282441 & 0.000015 & 0.282434 & -0.33 & 050 & 1136.9 & 20.49 & 1500.71 & 33.02 \\
\hline 13FR21\#8 & 715 & 0.008944 & 0.000401 & 0.282203 & 0.000014 & 0.282198 & -4.79 & & 1455.2 & 18.88 & 1916.95 & 30.65 \\
\hline 13FR21\#89 & 545 & 0.030526 & 0.001286 & 0.282080 & 0.000016 & & & 0.58 & 1663.0 & 22.97 & 2318.21 & 36.43 \\
\hline 13FR21\#90 & 492 & 0.022906 & 0.000884 & 0.282286 & 0.000013 & 0 & -6 & 0.47 & 1359.1 & 18.59 & 1882.13 & 29.80 \\
\hline 13FR21\#96 & 484 & 0.035261 & 0.001386 & 0.282556 & 0.000015 & 0.282543 & 2.23 & 0.53 & 995.4 & 21.23 & 1293.00 & 33.58 \\
\hline 13FR21\#98 & 537 & 026501 & 0.000978 & 0.2823 & 0.000016 & 0.282382 & -2.27 & 0.57 & 1214.1 & 22.44 & 619.84 & 5.89 \\
\hline \multicolumn{13}{|l|}{ OUT } \\
\hline OUT\#01 & 567 & 0.022677 & 0.000740 & & 9 & 0.281901 & $\begin{array}{c}- \\
18.65\end{array}$ & 1.74 & 1874.4 & 67.25 & 2671.22 & 08.24 \\
\hline OUT\# & 646 & 0.020079 & 0 & & 00052 & 0.281794 & $\begin{array}{c}- \\
20.67\end{array}$ & 1.85 & 2018.2 & 71.40 & 2857.42 & 115.07 \\
\hline OUT\#03 & 1924 & 0.019889 & D. & 0.281369 & 0.000062 & 0.281344 & -7.55 & 2.19 & 2607.5 & 83.53 & 3025.25 & 134.59 \\
\hline OUT\#05 & 565 & 0.020777 & 0.000697 & 0.282378 & 0.000051 & 0.282371 & -2.05 & 1.80 & 1224.4 & 70.59 & 1627.43 & 113 \\
\hline OUT\#06 & 2725 & 0.023026 & 0.000782 & 0.280956 & 0.000050 & 0.280916 & -4.12 & 1.79 & 3170.0 & 67.62 & 3440.43 & 108.7 \\
\hline OUT\#08 & 2633 & 0.042064 & 0.001323 & 0.280936 & 0.000051 & 0.280870 & -7.91 & 1.82 & 3242.1 & 69.61 & 3598.04 & 110.2 \\
\hline OUT\#09 & 513 & 0.046021 & 0.001497 & 0.282246 & 0.000052 & 0.282232 & -8.14 & 1.83 & 1437.5 & 73.16 & 1970.94 & 115.3 \\
\hline OUT\#10 & 1985 & 0.018426 & 0.000603 & 0.281293 & 0.000047 & 0.281271 & -8.73 & 1.66 & 2703.3 & 63.04 & 3145.18 & 101.8 \\
\hline OUT\#11 & 1928 & 0.029810 & 0.000990 & 0.281341 & 0.000047 & 0.281305 & -8.84 & 1.68 & 2665.9 & 64.28 & 3107.67 & 102.7 \\
\hline
\end{tabular}




\begin{tabular}{|c|c|c|c|c|c|c|c|c|c|c|c|c|}
\hline OUT\#15 & 545 & 0.045771 & 0.001684 & 0.282203 & 0.000060 & 0.282186 & -9.04 & 2.13 & 1505.6 & 85.23 & 2052.24 & 133.73 \\
\hline OUT\#16 & 496 & 0.021393 & 0.000705 & 0.282002 & 0.000046 & 0.281995 & 16.89 & 1.63 & 1744.7 & 63.39 & 2507.47 & 102.12 \\
\hline OUT\#17 & 824 & 0.036017 & 0.001344 & 0.282518 & 0.000051 & 0.282497 & 8.26 & 1.79 & 1047.8 & 71 & 1178.38 & 113.3 \\
\hline OUT\#18 & 1720 & 0.023858 & 0.000785 & 0.281314 & 0.000049 & 0.281289 & $\begin{array}{c}- \\
14.19\end{array}$ & 1.76 & 2687 & 66 & 3275.27 & 107.61 \\
\hline OUT\#19 & 544 & 0.020847 & 0.000793 & 0.282144 & 0.000051 & 0.282136 & 10.84 & 1.79 & & 69.87 & 2165.20 & 112.29 \\
\hline UT\#21 & 1954 & 0.023032 & 0.000778 & .282245 & 0.000047 & 0.282216 & 24.12 & 1.68 & 412.7 & 65.45 & 1083.70 & 105.2 \\
\hline $\mathrm{UT} \# 2 ?$ & 585 & 0.035320 & 0.001132 & 282066 & 0.000044 & 0.2 & & & 1675.3 & 61.70 & 2321.48 & 8.27 \\
\hline UT\#2 & 800 & 0.039878 & 0.001550 & 0.282600 & 0.000045 & 0.282 & 10.55 & 1.60 & 935.9 & 64.41 & 1013.95 & 101.43 \\
\hline OUT\#26 & 585 & 0.017435 & 0.000607 & 0.282054 & 0.000050 & 0.282047 & 13.07 & 1.78 & 1669.4 & 69.13 & 2336.33 & 111.6 \\
\hline OUT\#28 & 541 & 0.024558 & 0.000861 & 0.281824 & & 0.281816 & 22.24 & 1.67 & 1996.3 & 64.95 & 2875.65 & 104.19 \\
\hline OUT\#30 & 569 & 0.014808 & 0.000527 & 0.282212 & 0.000043 & 0.282206 & -7.79 & 1.52 & 1448.7 & 59.13 & 1992.83 & 95.70 \\
\hline OUT\#31 & 659 & 0.034401 & 0.001213 & 0.40 & 0.0 & 0.282236 & -4.70 & 1.60 & 1419.3 & 63.24 & 1867.22 & 100. \\
\hline OUT\#32 & 2998 & 0.021793 & 0.000720 & 0.280793 & 0.000048 & 0.280751 & -3.54 & 1.70 & 3383.8 & 63.64 & 3619.19 & 102.4 \\
\hline UT\#9 & 615 & 0.019161 & 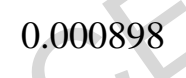 & 281779 & 0.000046 & 0.281769 & 22.24 & 1.63 & 2060.2 & 63.16 & 2931.16 & 101.23 \\
\hline OUT & 702 & 0 & 0. & 0.282225 & 0.000046 & 0.282209 & -4.70 & 1.63 & 1456.3 & 64.35 & 1900.87 & 102.2 \\
\hline OUT\#35 & 1919 & 0.020141 & 0.000718 & 0.281396 & 0.000041 & 0.281370 & -6.74 & 1.47 & 2572.7 & 56.13 & 2971.71 & 90.39 \\
\hline OUT\#3 & 2914 & 0.013784 & 0.000490 & 0.280875 & 0.000045 & 0.280848 & -2.08 & 1.58 & 3254.1 & 59.14 & 3464.99 & 95.82 \\
\hline OUT\#40 & 686 & 0.005939 & 0.000211 & 0.282257 & 0.000044 & 0.282254 & -3.47 & 1.56 & 1375.4 & 60.16 & 1811.15 & 98.18 \\
\hline OUT\#41 & 627 & 0.026727 & 0.001036 & 0.282193 & 0.000047 & 0.282181 & -7.39 & 1.68 & 1494.3 & 66.17 & 2011.83 & 105.66 \\
\hline OUT\#42 & 1941 & 0.024359 & 0.000843 & 0.281436 & 0.000044 & 0.281405 & -4.98 & 1.58 & 2526.7 & 60.36 & 2881.07 & 96.88 \\
\hline
\end{tabular}




\begin{tabular}{|c|c|c|c|c|c|c|c|c|c|c|c|c|}
\hline OUT\#43 & 1700 & 0.012944 & 0.000452 & 0.281418 & 0.000042 & 0.281404 & $\begin{array}{c}- \\
10.57\end{array}$ & 1.49 & 2525.1 & 56.58 & 3038.02 & 91.76 \\
\hline OUT\#44 & 581 & 0.033202 & 0.001279 & 0.281704 & 0.000052 & 0.281690 & 25.79 & 1.84 & 2185.3 & 72.11 & 3126.00 & 114.39 \\
\hline OUT\#45 & 1739 & 0.022115 & 0.000755 & 0.281780 & 0.000041 & 0.281755 & 2.79 & 1.46 & 2052.3 & 56.27 & 2243.04 & 90.52 \\
\hline OUT\#48 & 598 & 0.022045 & 0.000822 & 0.281863 & 0.000045 & 0.281854 & $\begin{array}{c}- \\
19.60\end{array}$ & 1.60 & & 62.10 & 2754.52 & 99.72 \\
\hline OUT\#50 & 1708 & 0.019002 & 0.000715 & 0.281271 & 0.000051 & 0.281248 & $\begin{array}{c}- \\
15.93\end{array}$ & & & 69.11 & 3372.84 & 111.30 \\
\hline OUT\#51 & 753 & 0.024068 & 0.000962 & 0.282488 & 0.000038 & 0.282474 & 5.83 & 1.35 & 1079.9 & 53.57 & 1276.06 & 85.71 \\
\hline OUT\#53 & 2175 & 0.010703 & 0.000475 & 0.281503 & 0.000040 & 0.281483 & 3.23 & 1.41 & 2412.0 & 53.42 & 2559.19 & 86.58 \\
\hline OUT\#54 & 843 & 0.017495 & 0.000682 & 0.282463 & 0.000043 & 0.282452 & 7.10 & 1.51 & 1105.9 & 59.23 & 1266.83 & 95.48 \\
\hline OUT\#98 & 2059 & 0.034965 & 0.001206 & 0.281582 & 0.000051 & 0.281535 & 2.37 & 1.81 & 2349.5 & 70.09 & 2520.80 & 111.41 \\
\hline OUT\#97 & 1896 & 0.024131 & 0.000886 & 0.281278 & 0.000052 & 0.281246 & $\begin{array}{c}- \\
11.65\end{array}$ & 1.83 & 2744.0 & 69.93 & 3255.43 & 112.11 \\
\hline OUT\#55 & 573 & 0.020714 & 0.000708 & 0.281967 & 0.000050 & 0.281959 & ${ }^{-}$ & 1.77 & 1793.3 & 68.52 & 2538.62 & 110.37 \\
\hline OUT\#56 & 639 & 0.031494 & 0.001010 & & 0.000044 & 0.282395 & 0.47 & 1.55 & 1194.2 & 61.31 & 1526.08 & 97.97 \\
\hline OUT\#61 & 2195 & 0.022630 & 0.000819 & 0.281381 & 0.000044 & 0.281347 & -1.16 & 1.58 & 2599.7 & 60.24 & 2844.96 & 96.75 \\
\hline OUT\#57 & 1935 & 0.026001 & 0.000913 & 0.281467 & 0.000043 & 0.281433 & -4.11 & 1.53 & 2488.9 & 58.73 & 2822.41 & 94.09 \\
\hline OUT\#58 & 586 & 0.03 & 0.001181 & 0.281954 & 0.000045 & 0.281941 & ${ }^{-}$ & 1.58 & 1832.7 & 62.11 & 2568.89 & 98.79 \\
\hline OUT\#62 & 2370 & 0.032075 & 0.00129 & 0.281344 & 0.000052 & 0.281286 & 0.74 & 1.84 & 2682.6 & 71.14 & 2865.73 & 112.82 \\
\hline OUT\#60 & 576 & 0.031211 & 0.001146 & 0.282105 & 0.000044 & 0.282093 & $\begin{array}{c}- \\
11.64\end{array}$ & 1.56 & 1620.8 & 61.26 & 2239.35 & 97.53 \\
\hline OUT\#63 & 564 & 0.013354 & 0.000539 & 0.282206 & 0.000046 & 0.282200 & -8.12 & 1.64 & 1457.5 & 63.79 & 2009.64 & 103.21 \\
\hline OUT\#64 & 569 & 0.018154 & 0.000653 & 0.281939 & 0.000046 & 0.281932 & 17.51 & 1.62 & 1829.1 & 62.62 & 2601.62 & 101.01 \\
\hline OUT\#65 & 2127 & 0.013426 & 0.000489 & 0.281374 & 0.000046 & 0.281355 & -2.46 & 1.64 & 2586.5 & 62.17 & 2871.38 & 100.7 \\
\hline
\end{tabular}




\begin{tabular}{|c|c|c|c|c|c|c|c|c|c|c|c|c|}
\hline OUT\#66 & 886 & 0.028647 & 0.001096 & 0.282584 & 0.000045 & 0.282566 & 12.09 & 1.60 & 947.7 & 63.79 & 984.38 & 101. \\
\hline UT\#69 & 632 & 0.020252 & 000743 & 0.282403 & 0.000046 & 0.282394 & 0.29 & 1.61 & 1191.0 & 63.33 & 1531.52 & 101. \\
\hline OUT\#70 & 489 & 0.038551 & 0.001429 & .282423 & 0.000049 & 0.282410 & -2.36 & 1.73 & 1184.3 & 69.24 & 587.61 & 109.4 \\
\hline OUT\#72 & 650 & 0.012297 & 0.000419 & 0.282175 & 0.000047 & 0.282170 & -7.26 & 1.66 & & 64.28 & 2021.94 & 104.3 \\
\hline OUT\#74 & 544 & 0.018104 & 0.000612 & 0.282046 & 0.000041 & 0.282040 & $\overline{14.24}$ & 1.45 & 67 & 56.15 & 2378.10 & 90.67 \\
\hline UT\#75 & 2632 & 0.026002 & 0.000915 & 0.281026 & 0.000053 & 0.280980 & -4.02 & & & 71.85 & 3361.56 & 115.10 \\
\hline UTस76 & 0 & 0.012151 & 0000448 & .282233 & 0000056 & 30 & 12.98 & 7 & 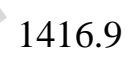 & 76.65 & 2112.11 & 2123 \\
\hline UT\#77 & 628 & 0.031051 & 0.001038 & 0.282150 & 0.000045 & 0.2 & 00 & 1.60 & 1554.0 & 62.95 & 2106.44 & 100.5 \\
\hline UT\#78 & 629 & 0.020074 & 0.000684 & 0.282099 & 0 & & 10.52 & 1.60 & 1609.8 & 62.30 & 2209.94 & 100.4 \\
\hline OUT\#81 & 1904 & 0.024953 & 0.000851 & 0.281421 & 0.000050 & 0.281391 & -6.34 & 1.77 & 2546.8 & 67.75 & 2935.48 & 108.71 \\
\hline OUT\#82 & 593 & 0.025706 & 0.000901 & 0.281833 & 0.000048 & 0.281823 & 20.81 & 1.69 & 1986.3 & 65.61 & 2825.84 & 105.1 \\
\hline OUT\#83 & 662 & 0.018852 & 0.000629 & 0.282331 & 0.000046 & 0.282323 & -1.57 & 1.64 & 1288.0 & 64.06 & 1672.38 & 103.4 \\
\hline OUT\#84 & 1629 & 0.025816 & 0.000880 & & 0.000046 & 0.281606 & -5.03 & 1.62 & 2260.2 & 62.32 & 2641.14 & 99.92 \\
\hline OUT\#85 & 495 & 0.041533 & 0.001380 & 0.282379 & 0.000049 & 0.282367 & -3.77 & 1.74 & 1245.1 & 69.39 & 1681.75 & 109.7 \\
\hline OUT\#86 & 611 & 0.024518 & 0.000 & 0.282478 & 0.000041 & 0.282469 & 2.45 & 1.46 & 1088.9 & 57.39 & 1378.96 & 92.23 \\
\hline OUT & 690 & 0 & 0 & 0.2 & 0.0 & 0.2 & 7.39 & 1.78 & 9 & 70.20 & 112 & 112.8 \\
\hline OUT\#90 & 722 & 0.013453 & 0.000521 & 0.282458 & 0.000049 & 0.282451 & 4.32 & 1.73 & 1108.4 & 67.76 & 1347.41 & 109.69 \\
\hline OUT\#91 & 795 & 0.053931 & 0.001822 & 0.282602 & 0.000045 & 0.282574 & 10.33 & 1.61 & 941.2 & 65.35 & 1023.56 & 102.1 \\
\hline OUT\#92 & 609 & 0.027019 & 0.000897 & 0.282204 & 0.000050 & 0.282194 & -7.32 & 1.77 & 1473.0 & 69.58 & 1993.68 & 111.52 \\
\hline OUT\#93 & 603 & 0.027858 & 0.000904 & 0.282373 & 0.000046 & 0.282363 & -1.49 & 1.62 & 1238.6 & 64.01 & 1621.55 & 102.5 \\
\hline OUT\#95 & 1168 & 0.047493 & 0.001714 & 0.282469 & 0.000061 & 0.282431 & 13.71 & 2.18 & 1128.7 & 87.84 & 1106.43 & 137.7 \\
\hline
\end{tabular}




\begin{tabular}{|c|c|c|c|c|c|c|c|c|c|c|c|c|}
\hline OUT\#100 & 152 & 0.012875 & 0.000422 & 0.281262 & 0.000047 & 0.281244 & -5.80 & 1.68 & 2733.4 & 63.33 & 3095.65 & 2.79 \\
\hline \multicolumn{13}{|l|}{ MB2 } \\
\hline IB2\#2 & 962 & .014090 & 0.000474 & 0.281275 & 0.000044 & 0.281257 & -9.75 & 1.57 & 2719.6 & 59.21 & 3189.94 & 95.96 \\
\hline MB2\#3 & 584 & 0.016724 & 0.000535 & 0.281821 & 0.000048 & 0.281816 & 21.28 & 1.71 & 1983.6 & 3 & 2848.77 & 06.52 \\
\hline MB2\#4 & 1872 & 0.015299 & 0.000483 & 0.281283 & 0.000051 & 0.281266 & $\begin{array}{c}- \\
11.50\end{array}$ & 1.80 & & 7.99 & 3227.88 & 110.17 \\
\hline MB2\#5 & 557 & 0.034845 & 0.001005 & 0.282295 & 0.000046 & 0.282284 & -5.30 & 1.63 & 1351.3 & 64.21 & 1826.34 & 02.61 \\
\hline MB2\#7 & 646 & 0.016550 & 0.000590 & 0.282307 & 0.000041 & 0.282299 & -2.76 & 1.45 & 1320.0 & 56.59 & 1735.05 & 91.44 \\
\hline MB2\#8 & 1994 & 0.008175 & 0.000269 & 0.281056 & 0.000048 & 0.28 & & 1.71 & 2996.5 & 63.79 & 3627.56 & 103.95 \\
\hline MB2\#9 & 1725 & 0.007678 & 0.000246 & 0.281462 & 0.000046 & 0.281 & -8.20 & 1.64 & 2452.6 & 61.90 & 2911.39 & 100.93 \\
\hline MB2\#10 & 559 & 0.012864 & 0.000418 & 0.282081 & 0.000046 & 0.282077 & 12.58 & 1.62 & 1623.1 & 62.38 & 2286.12 & 01.25 \\
\hline MB2\#12 & 576 & 0.021837 & 0.000703 & 0.282303 & 0.000047 & 0.282295 & -4.48 & 1.65 & 1329.0 & 64.46 & 1789.16 & 103.85 \\
\hline MB2\#13 & 654 & 0.030648 & 0.001017 & 0.281914 & 0.000049 & 0.281901 & $\begin{array}{c}- \\
16.68\end{array}$ & 1.74 & 1881.3 & 68.01 & 2614.68 & 108.65 \\
\hline MB2\#14 & 486 & 0.022402 & 0.000800 & 0.282321 & 0.000046 & 0.282314 & -5.85 & 1.61 & 1307.5 & 63.24 & 1806.01 & 101.62 \\
\hline MB2\#16 & 2232 & 0.016221 & 0.000607 & 0.281381 & 0.000045 & 0.281356 & 0.01 & 1.61 & 2584.9 & 60.99 & 2802.02 & 98.50 \\
\hline MB2\#17 & 818 & 0.028990 & 0.001056 & 0.282505 & 0.000046 & 0.282489 & 7.83 & 1.63 & 1057.9 & 64.57 & 1200.93 & 103.05 \\
\hline MB2\#18 & 640 & 0.010474 & 0.000339 & 0.282321 & 0.000045 & 0.282317 & -2.26 & 1.59 & 1291.1 & 61.76 & 1699.10 & 100.4 \\
\hline MB2\#19 & 2088 & 0.028988 & 0.001054 & 0.281377 & 0.000046 & 0.281335 & -4.05 & 1.64 & 2620.9 & 63.10 & 2938.43 & 100.71 \\
\hline MB2\#20 & 735 & 0.018397 & 0.000637 & 0.282035 & 0.000049 & 0.282026 & 10.42 & 1.73 & 1695.8 & 66.93 & 2285.53 & 108.01 \\
\hline MB2\#21 & 595 & 0.020980 & 0.000683 & 0.282303 & 0.000052 & 0.282295 & -4.05 & 1.85 & 1328.4 & 72.14 & 1777.14 & 116.27 \\
\hline MB2\#24 & 644 & 0.016611 & 0.000584 & 0.282398 & 0.000049 & 0.282391 & 0.43 & 1.72 & 1193.6 & 67.26 & 1532.27 & 108.69 \\
\hline MB2\#25 & 1983 & 0.024827 & 0.000809 & 0.281421 & 0.000049 & 0.281391 & -4.52 & 1.76 & 2544.6 & 67.10 & 2885.15 & 107.80 \\
\hline
\end{tabular}




\begin{tabular}{|c|c|c|c|c|c|c|c|c|c|c|c|c|}
\hline MB2\#26 & 38 & 0.017119 & 0.000552 & 0.282238 & 0.000043 & 0.282231 & -5.36 & 1.53 & 1413.7 & 59.36 & 1892.63 & .01 \\
\hline MB2\#27 & 636 & 0.042009 & 0.001380 & 0.282220 & 0.000057 & 0.282203 & -6.39 & 2.03 & 1470.5 & 80.62 & 1956.20 & 127.55 \\
\hline $1 \mathrm{~B} 2 \# 28$ & 1353 & 0.017748 & 0.000592 & 0.281442 & 0.000039 & 0.281426 & $\begin{array}{c}- \\
17.70\end{array}$ & 1.39 & 2502.6 & 52.83 & 3210.39 & 5.35 \\
\hline MB2\#29 & 069 & 0.012646 & 0.000422 & 0.281499 & 0.000045 & 0.281483 & 0.75 & 1.60 & 241 & 60.69 & 2628.43 & 98.49 \\
\hline MB2\#31 & 1960 & 0.018208 & 0.000591 & 0.281444 & 0.000048 & 0.281422 & -3.93 & 1.71 & 2499.3 & 65.12 & 2831.40 & 105.22 \\
\hline 1B2\#33 & 642 & 0.020353 & ו ו & 0.282478 & 0.000051 & 0.282470 & 3.18 & 10 & & 71.01 & 1356.57 & 114.41 \\
\hline MB2\#35 & 1916 & 0.016123 & 0.000537 & 0.281435 & 0.000044 & 0.281415 & -5.19 & 1.58 & 2508.1 & 59.85 & 2874.03 & 96.84 \\
\hline MB2\#36 & 720 & 0.019549 & 0.000761 & 0.282567 & 0.000046 & 0.282556 & 8.00 & 1.63 & 963.9 & 64.26 & 112.30 & 103.36 \\
\hline MB2\#37 & 674 & 0.023196 & 0.000820 & 0.282182 & 0.000046 & 0.282172 & -6.64 & 1.64 & 1500.5 & 64.16 & 2001.36 & 103.0 \\
\hline 1B2\#39 & 1856 & 0.0215 & 0.000769 & 0.281547 & 0.000052 & 0.281520 & -2.87 & 1.85 & 2371.2 & 70.83 & 2684.53 & 113.91 \\
\hline MB2\#40 & 2221 & 0.019654 & 0.000677 & 0.281399 & 0.000043 & 0.281370 & 0.26 & 1.53 & 2566.3 & 58.13 & 2777.72 & 93.70 \\
\hline MB2\#41 & 762 & 0.014601 & 0.000521 & 0.282535 & 0.000 & 0.282528 & 7.94 & 1.38 & 1001.4 & 54.13 & 1149.46 & 87.62 \\
\hline MB2\#43 & 609 & 0.033812 & 0.001187 & 0.282210 & 0.000052 & 0.282196 & -7.23 & 1.83 & 1476.3 & 72.13 & 1988.23 & 114.7 \\
\hline MB2\#44 & 1889 & 0.022299 & 0.000783 & 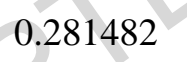 & 0.000042 & 0.281454 & -4.44 & 1.51 & 2460.1 & 57.76 & 2807.06 & 92.86 \\
\hline IB2\#46 & 2013 & 0.026153 & 0.000 & 0.281500 & 0.000050 & 0.281467 & -1.10 & 1.76 & 2440.3 & 67.55 & 2698.53 & 108.3 \\
\hline MB2\#47 & 1642 & 0.013672 & & 0.281805 & 0.000042 & 0.281788 & 1.74 & 1.51 & 2006.2 & 57.79 & 2231.53 & 93.53 \\
\hline MB2\#48 & 587 & 0.012174 & 0 & 0.282069 & 0.000048 & 0.282064 & $\begin{array}{c}- \\
12.41\end{array}$ & 1.72 & 1640.8 & 66.27 & 2296.26 & 107.49 \\
\hline MB2\#49 & 675 & 0.020773 & 0.000724 & 0.282044 & 0.000052 & 0.282035 & $\begin{array}{c}- \\
11.48\end{array}$ & 1.86 & 1687.9 & 72.18 & 2305.88 & 116.22 \\
\hline MB2\#50 & 822 & 0.029464 & 0.001036 & 0.282440 & 0.000047 & 0.282424 & 5.63 & 1.66 & 1148.3 & 65.84 & 1343.05 & 105.1 \\
\hline [B2 & 592 & 0 & 0.0 & 0.2 & 0. & 0.2 & $\begin{array}{c}- \\
17.87\end{array}$ & 1.55 & .1 & 61.15 & 264 & 96.70 \\
\hline IB2\#52 & 1796 & 0.012833 & 0.000420 & 0.281448 & 0.000040 & 0.281433 & -7.31 & 1.43 & 2483.4 & 54.34 & 2911.67 & 8. \\
\hline
\end{tabular}




\begin{tabular}{|c|c|c|c|c|c|c|c|c|c|c|c|c|}
\hline MB2\#53 & 2649 & 0.028095 & 0.000952 & 0.281067 & 0.000046 & 0.281019 & -2.22 & 1.62 & 3034.4 & 61.67 & 265.81 & 98.70 \\
\hline MB2\#54 & 658 & 0.014850 & 0.000533 & 0.282364 & 0.000042 & 0.282357 & -0.44 & 1.49 & 1238.8 & 57.96 & 1597.96 & 93.79 \\
\hline MB2\#55 & 586 & 0.016824 & 0.000692 & 0.282160 & 0.000049 & 0.282152 & -9.32 & 1.72 & 1526.6 & 7.17 & 101.76 & 08.24 \\
\hline MB2\#56 & 1865 & 0.021591 & 0.000720 & 0.281290 & 0.000040 & 0.281265 & $\begin{array}{c}- \\
11.70\end{array}$ & 1.42 & & & 234.37 & 6.83 \\
\hline MB2\#57 & 552 & 0.032831 & 0.001126 & 0.282003 & 0.000048 & 0.281991 & $\begin{array}{c}- \\
15.77\end{array}$ & 1.69 & & 2 & 2480.06 & 105.80 \\
\hline IB2\#58 & 649 & 0.031234 & 0.001139 & 0.281969 & 0.000045 & 0.281955 & 14.90 & & & 62.29 & 499.44 & 9.19 \\
\hline IB2\#59 & 2211 & 0.014084 & 0.000490 & 0.281254 & 0.000043 & 0.281233 & 402 & 1.54 & 2748.8 & 58.08 & 3082.34 & 94.09 \\
\hline MB2\#60 & 635 & 0.034871 & 0.001284 & 0.282299 & 0.000047 & 0.28 & -3 & 1.67 & 1354.8 & 66.38 & 1776.79 & 105.29 \\
\hline IB2\#61 & 1868 & 0.015793 & 0.000561 & 0.281451 & 0.000047 & 0.28 & -5.73 & 1.68 & 2487.6 & 63.77 & 2869.89 & 103.11 \\
\hline MB2\#63 & 2717 & 0.018748 & 0.000645 & 0.281075 & 0.000044 & 0.281042 & 0.18 & 1.56 & 2999.9 & 58.79 & 3173.38 & 94.86 \\
\hline IB2\#65 & 630 & 0.026389 & 0.000967 & 0.282240 & 0.000049 & 0.282229 & -5.61 & 1.74 & 1425.6 & 68.60 & 1902.65 & 109.74 \\
\hline IB2\#66 & 1962 & 0.014551 & 0.000531 & 0.281286 & 0.000047 & 0.281266 & -9.43 & 1.65 & 2708.8 & 62.55 & 3170.52 & 101.23 \\
\hline IB2\#67 & 605 & 0.025540 & 0.00 & & 41 & 0.282045 & $\begin{array}{c}- \\
12.70\end{array}$ & 1.44 & 1679.7 & 56.12 & 2328.37 & 89.99 \\
\hline IB2\#68 & 603 & 0.0 & 0 & 0.282156 & 0.000040 & 47 & -9.13 & 1.40 & 7.2 & 4.72 & 3.12 & 87.86 \\
\hline MB2\#70 & 1973 & 0.016320 & 0. & 0.281383 & 0.000044 & 0.281361 & -5.81 & 1.56 & 2581.8 & 59.25 & 2956.50 & 95.73 \\
\hline 17 & 581 & 0 & & 1756 & 0.000052 & 0.281741 & 24.00 & 1.84 & 2119.5 & 72.41 & 3014.64 & 114.54 \\
\hline MB2\#73 & 1909 & 0.016952 & 0.000623 & 0.281446 & 0.000047 & 0.281423 & -5.07 & 1.66 & 2498.8 & 63.09 & 2861.21 & 101.85 \\
\hline MB2\#74 & 2113 & 0.006606 & 0.000265 & 0.281368 & 0.000043 & 0.281357 & -2.69 & 1.53 & 2580.3 & 57.50 & 2874.62 & 93.70 \\
\hline MB2\#75 & 642 & 0.027574 & 0.001025 & 0.281739 & 0.000050 & 0.281727 & 23.12 & 1.79 & 2122.2 & 69.45 & 3006.21 & 110.93 \\
\hline IB2\#77 & 569 & 0.015860 & 0.000672 & 0.282196 & 0.000051 & 2189 & -8.40 & 1.81 & 147 & 70.47 & 031.09 & 10 \\
\hline MB2\#79 & 1940 & 0.008649 & 0.000316 & 0.281238 & 0.000045 & 0.281226 & - & 1.59 & 2757.5 & 59.69 & 3270.47 & 97.15 \\
\hline
\end{tabular}




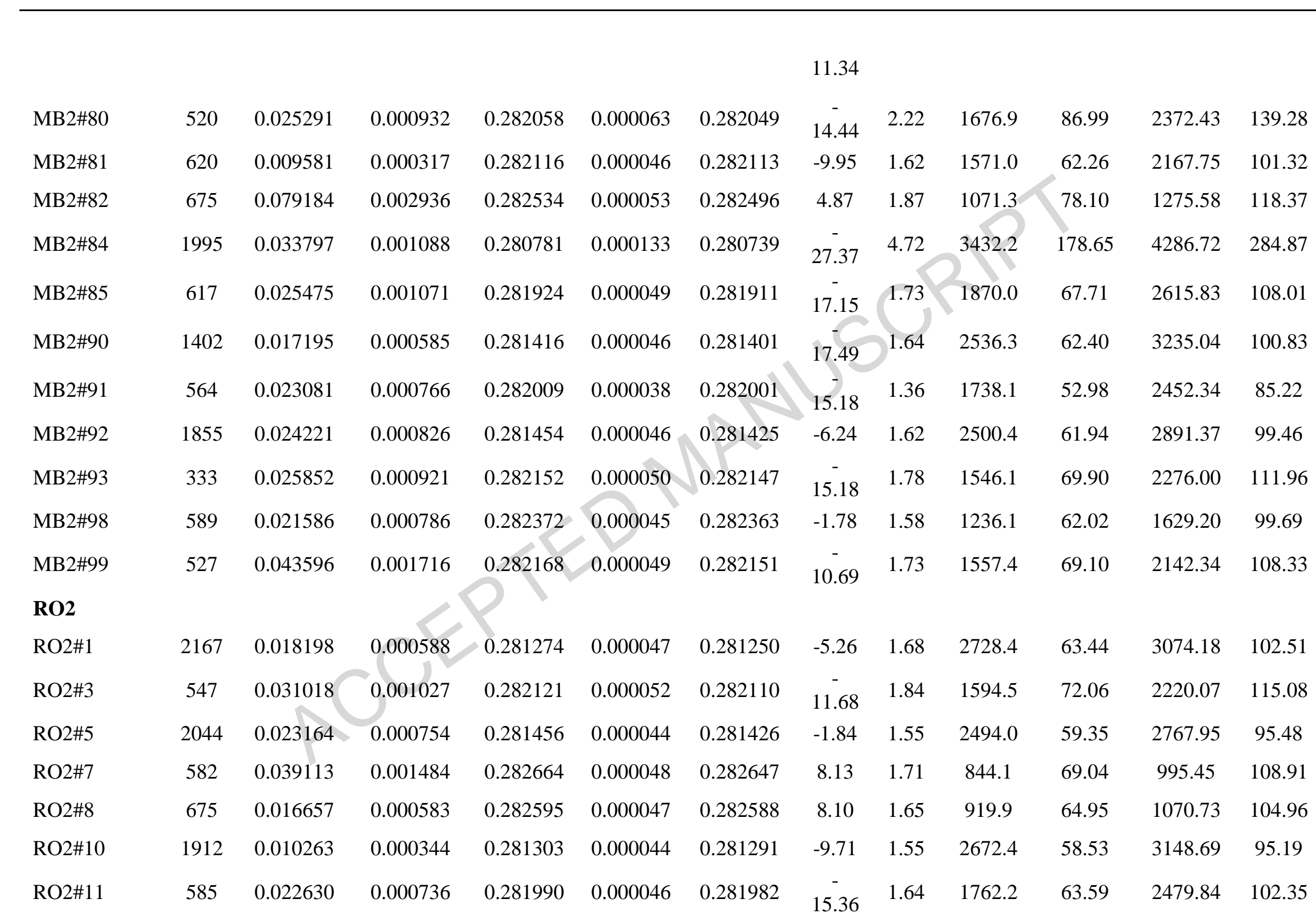




\begin{tabular}{|c|c|c|c|c|c|c|c|c|c|c|c|c|}
\hline RO2\#12 & 566 & 0.036324 & 0.001212 & 0.282425 & 0.000047 & 0.282412 & -0.56 & 1.65 & 1175.2 & 65.74 & 1534.18 & 104.48 \\
\hline RO2\#13 & 1741 & 0.031242 & 0.001074 & 0.281682 & 0.000049 & 0.281647 & -1.01 & 1.75 & 2204.0 & 67.94 & 2479.49 & 108.38 \\
\hline RO2\#14 & 1924 & 0.039966 & 0.001590 & 0.282483 & 0.000068 & 0.282425 & 30.86 & 2.40 & 1104.6 & 96.28 & 634.94 & 151.46 \\
\hline RO2\#15 & 1924 & 0.036936 & 0.001299 & 0.281404 & 0.000044 & 0.281356 & -7.09 & 1.57 & 2601.0 & 60.80 & 2997.40 & 96.40 \\
\hline RO2\#17 & 3300 & 0.016168 & 0.000548 & 0.280543 & 0.000043 & 0.280508 & -5.05 & 1.55 & 3699.9 & 57.28 & 3946.84 & 92.66 \\
\hline RO2\#18 & 2638 & 0.022313 & 0.000700 & 0.281078 & 0.000048 & 0.281042 & -1.65 & 1.72 & 3000.8 & 65.09 & 3222.55 & 104.86 \\
\hline RO2\#26 & 647 & 0.020229 & 0.000781 & 0.282144 & 0.000043 & 0.282135 & -8.57 & 1.53 & 1551.9 & 59.61 & 2101.75 & 95.84 \\
\hline RO2\#35 & 1828 & 0.016683 & 0.000570 & 0.281789 & 0.000047 & 0.281769 & 5.35 & 1.65 & 2029.7 & 63.36 & 2154.11 & 102.44 \\
\hline RO2\#41 & 1265 & 0.035987 & 0.001244 & 0.282145 & 0.000054 & 0.282115 & 4.73 & 1.90 & 1569.5 & 75.03 & 1748.46 & 119.14 \\
\hline RO2\#43 & 950 & 0.034820 & 0.000872 & 0.281822 & 0.000324 & 0.281806 & 13.38 & 11.47 & 2000.7 & 443.03 & 2635.05 & 710.51 \\
\hline RO2\#44 & 1902 & 0.018597 & 0.000681 & 0.281336 & 0.000044 & 0.281312 & -9.19 & 1.56 & 2651.0 & 59.48 & 3109.29 & 95.88 \\
\hline RO2\#46 & 649 & 0.030006 & 0.001163 & 0.282386 & 0.000047 & 0.282372 & -0.12 & 1.66 & 1228.5 & 65.86 & 1571.10 & 104.81 \\
\hline $\mathrm{RO} 2 \# 48$ & 590 & 0.018412 & 0.000665 & 0281070 & 0.000043 & 0.281962 & $\begin{array}{c}- \\
15.96\end{array}$ & 1.51 & 1787.3 & 58.51 & 2520.72 & 94.36 \\
\hline RO2\#50 & 447 & 0.046705 & 0.001603 & 0.282114 & 0.000078 & 0.282101 & $\begin{array}{c}- \\
14.25\end{array}$ & 2.75 & 1628.1 & 109.49 & 2304.34 & 172.17 \\
\hline RO2\#53 & 2135 & 0.014022 & 0.000523 & 0.281371 & 0.000045 & 0.281350 & -2.44 & 1.59 & 2593.1 & 60.39 & 2876.55 & 97.75 \\
\hline RO2\#57 & 632 & 0.023949 & 0.000868 & 0.282144 & 0.000045 & 0.282134 & -8.94 & 1.59 & 1555.6 & 62.29 & 2113.37 & 99.92 \\
\hline RO2\#58 & 2250 & 0.008765 & 0.000341 & 0.281363 & 0.000043 & 0.281349 & 0.18 & 1.53 & 2591.6 & 57.85 & 2805.58 & 94.08 \\
\hline RO2\#59 & 547 & 0.126595 & 0.004627 & 0.282430 & 0.000058 & 0.282382 & -2.05 & 2.04 & 1285.6 & 88.97 & 1612.99 & 128.41 \\
\hline RO2\#63 & 526 & 0.054199 & 0.001806 & 0.282145 & 0.000052 & 0.282127 & 11.56 & 1.84 & 1593.8 & 73.88 & 2195.86 & 115.54 \\
\hline RO2\#65 & 584 & 0.013194 & 0.000468 & 0.281539 & 0.000047 & 0.281534 & $\begin{array}{c}- \\
31.25\end{array}$ & 1.65 & 2362.9 & 63.09 & 3467.70 & 102.26 \\
\hline RO2\#71 & 2113 & 0.029633 & 0.001055 & 0.281281 & 0.000066 & 0.281239 & -6.91 & 2.35 & 2752.1 & 90.09 & 3133.03 & 143.78 \\
\hline
\end{tabular}




\begin{tabular}{|c|c|c|c|c|c|c|c|c|c|c|c|c|}
\hline RO2\#73 & 2162 & 0.035157 & 0.001179 & 0.281351 & 0.000039 & 0.281302 & -3.52 & 1.40 & 2665.8 & 53.98 & 2963.36 & 85.86 \\
\hline RO2\#76 & 679 & 0.018786 & 0.000623 & 0.282196 & 0.000048 & 0.282188 & -5.95 & 1.69 & 1473.7 & 65.63 & 1961.89 & 105.96 \\
\hline RO2\#77 & 2063 & 0.016134 & 0.000586 & 0.281437 & 0.000048 & 0.281414 & -1.85 & 1.70 & 2509.0 & 64.80 & 2783.75 & 104.71 \\
\hline RO2\#78 & 596 & 0.019816 & 0.000742 & 0.282180 & 0.000043 & 0.282172 & -8.41 & 1.53 & 1500.8 & 59.66 & 2052.05 & 96.01 \\
\hline RO2\#79 & 1915 & 0.017743 & 0.000612 & 0.281458 & 0.000046 & 0.281436 & -4.47 & 1.65 & 2481.1 & 62.86 & 2829.19 & 101.51 \\
\hline RO2\#80 & 580 & 0.034153 & 0.001180 & 0.282093 & 0.000047 & 0.282080 & 12.00 & & & 65.76 & 2265.24 & 104.59 \\
\hline RO2\#81 & 632 & 0.022725 & 0.000808 & 0.281801 & 0.000048 & 0.281791 & 21.06 & 1.69 & 2025.7 & 65.55 & 2870.93 & 105.30 \\
\hline RO2\#82 & 2529 & 0.010309 & 0.000359 & 0.280974 & 0.000044 & 0.280957 & -7.23 & 1.57 & 3111.8 & 58.46 & 3476.50 & 95.04 \\
\hline RO2\#83 & 1975 & 0.014028 & 0.000458 & 0.281422 & 0.000050 & 0.281405 & -4.20 & 1.77 & 2520.3 & 67.18 & 2859.12 & 108.93 \\
\hline RO2\#87 & 2192 & 0.072759 & 0.002466 & 0.281351 & 0.000053 & 0.281248 & -4.73 & 1.87 & 2757.7 & 74.62 & 3060.67 & 114.58 \\
\hline RO2\#88 & 621 & 0.021816 & 0.000728 & 0.281661 & 0.000045 & 0.281652 & $\begin{array}{c}- \\
26.23\end{array}$ & 1.60 & 2213.1 & 61.71 & 3184.15 & 99.34 \\
\hline RO2\#92 & 2068 & 0.019264 & 0.000663 & 0.281478 & 0.000047 & 0.281452 & -0.36 & 1.68 & 2457.4 & 64.15 & 2696.00 & 103.46 \\
\hline
\end{tabular}




\section{Highlights:}

- In the St-Georges-sur-Loire block-in-matrix unit, detrital zircon ages spectra and their Hf isotopic composition predominantly indicate Early Paleozoic and Neoproterozoic populations.

- The analyzed zircon grains are preferentially derived from oa southern source, but at northern one cannot be discarded

- Most of the grains record negative Hf isotopic values indicating that crustal melting played a major role in the Neoproterozoic and Paleozoic evolution of Armorica microcontinent. 


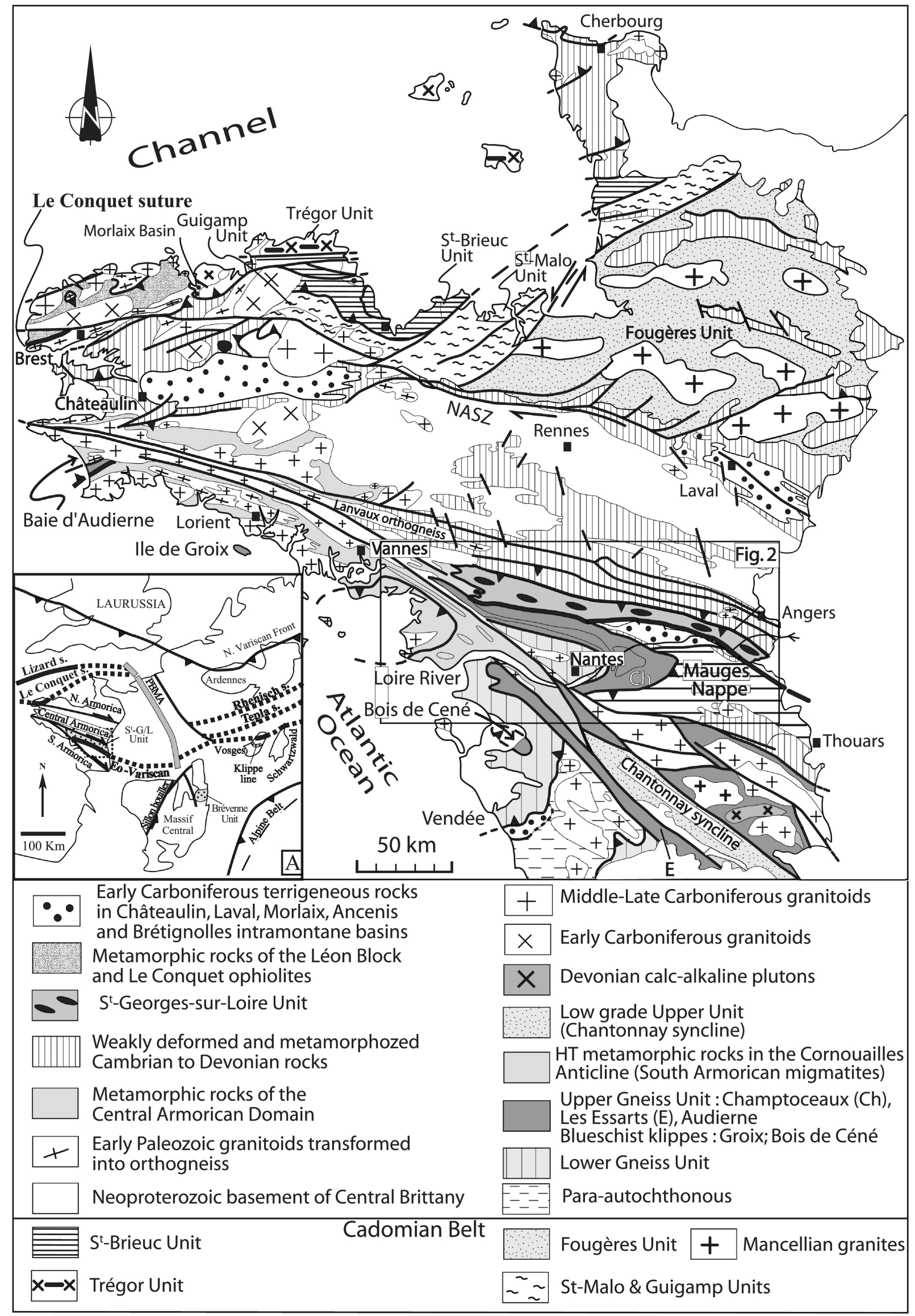




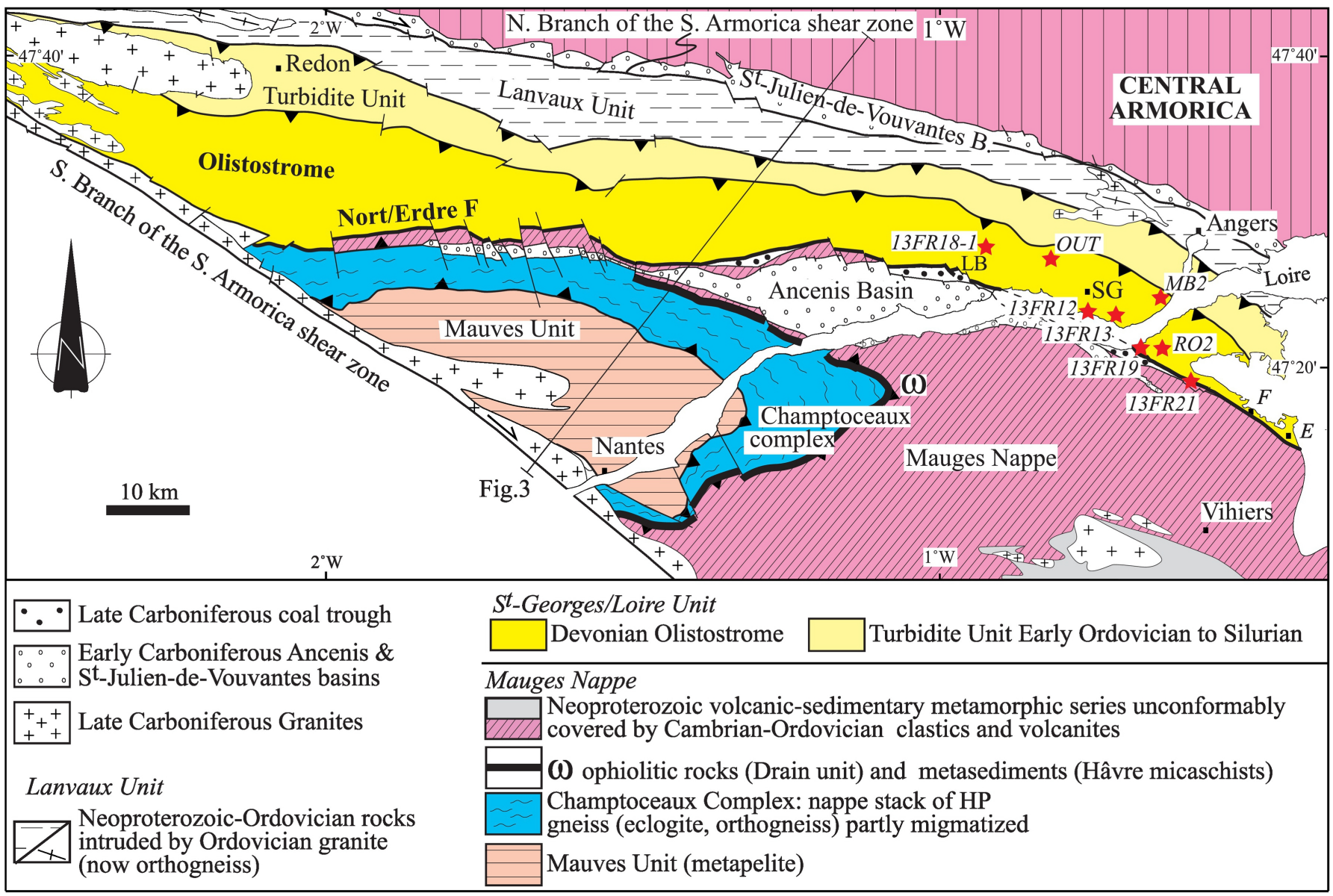


NE Armorica

North Gondwana Margin

\section{Nort-sur-Erdre Fault (Eo-Variscan suture)}

St-Georges/Loire Unit

Lanvaux Unit

North Branch of the South Armorican shear Zone

Neoproterozoic $\oplus$ rocks

Unknown Proterozoic basement

Mauges Nappe

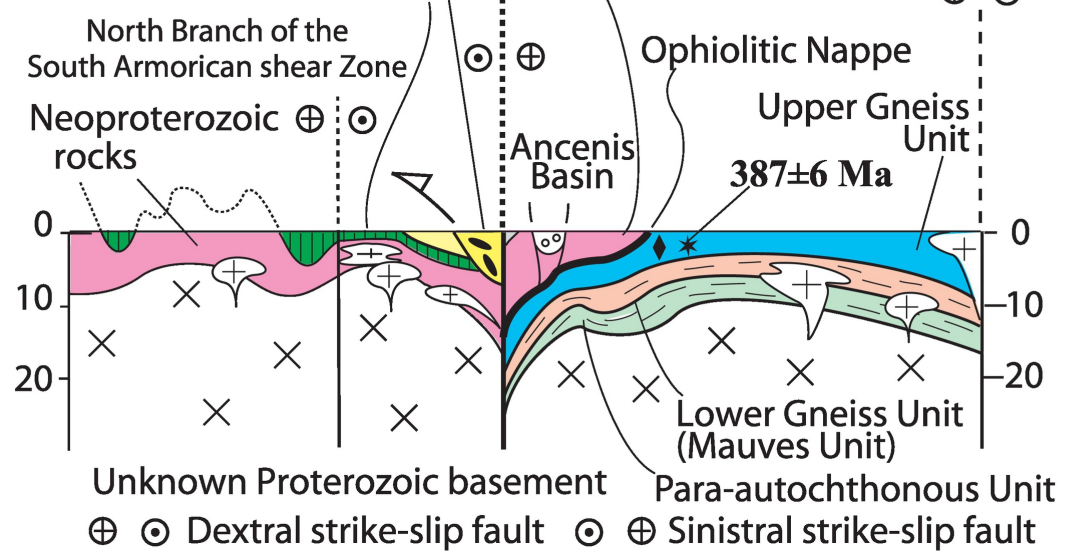

South Branch of the South Armorican shear Zone

$\oplus$, $\odot$ 


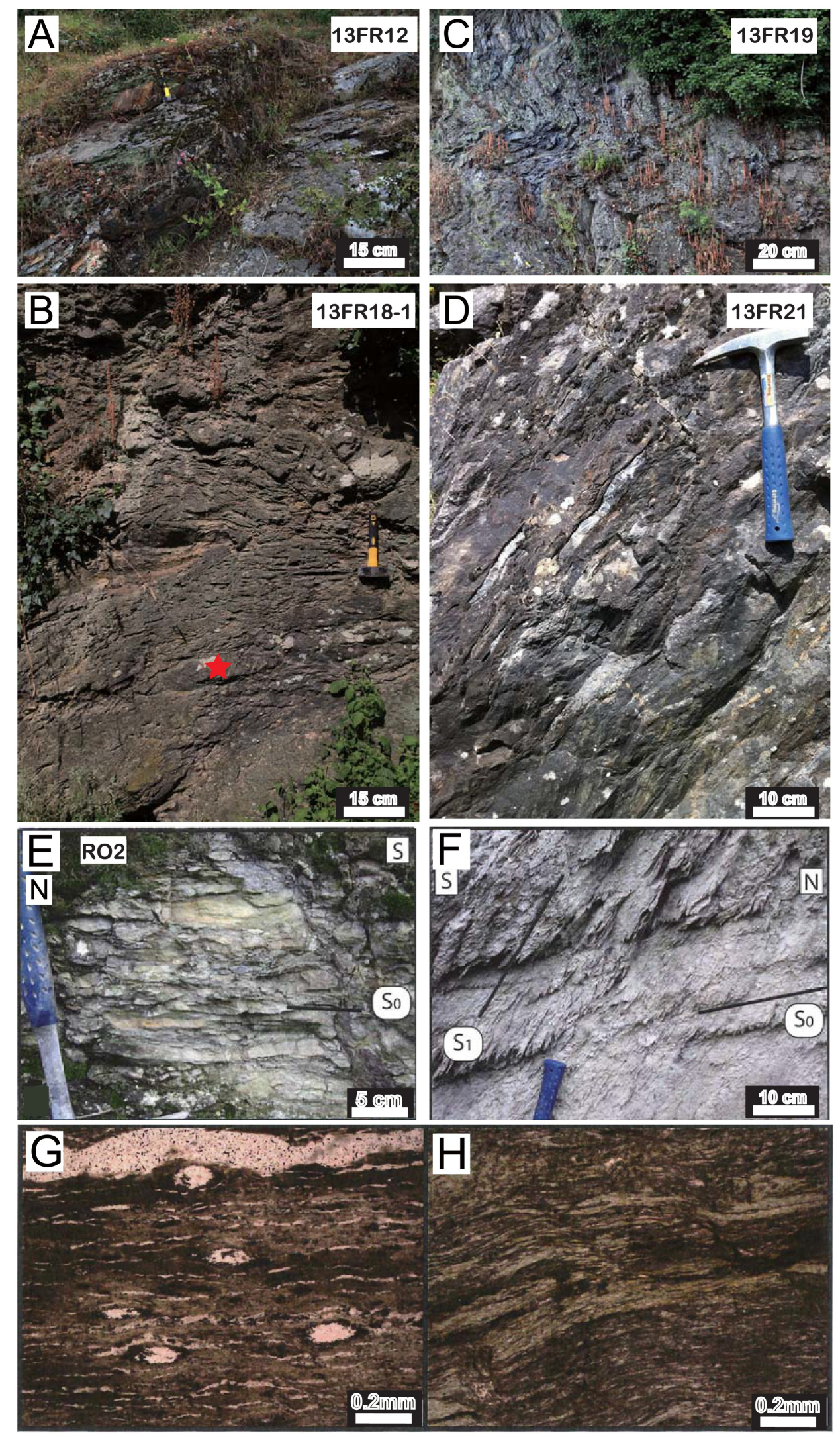



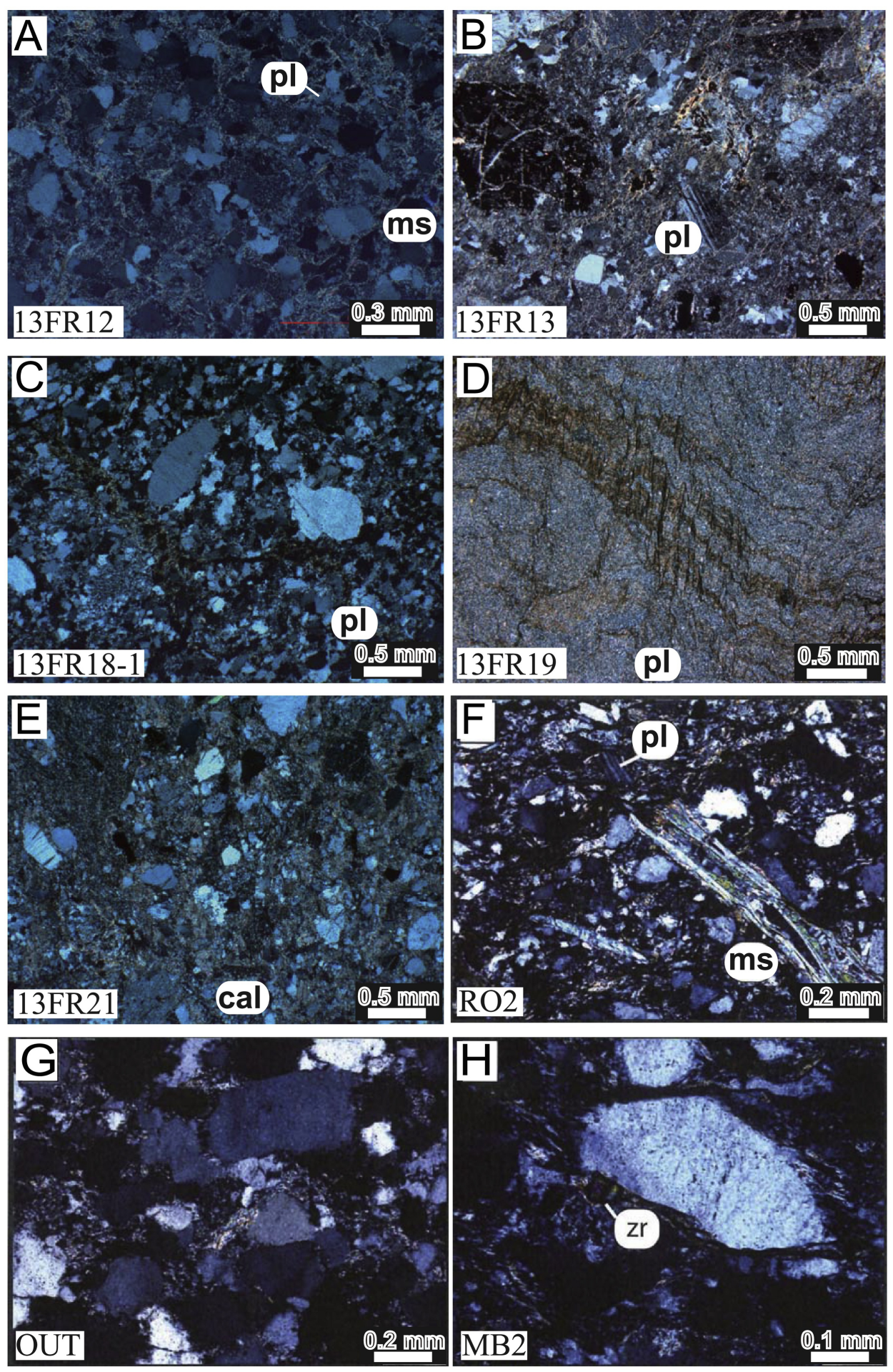

Figure 5 


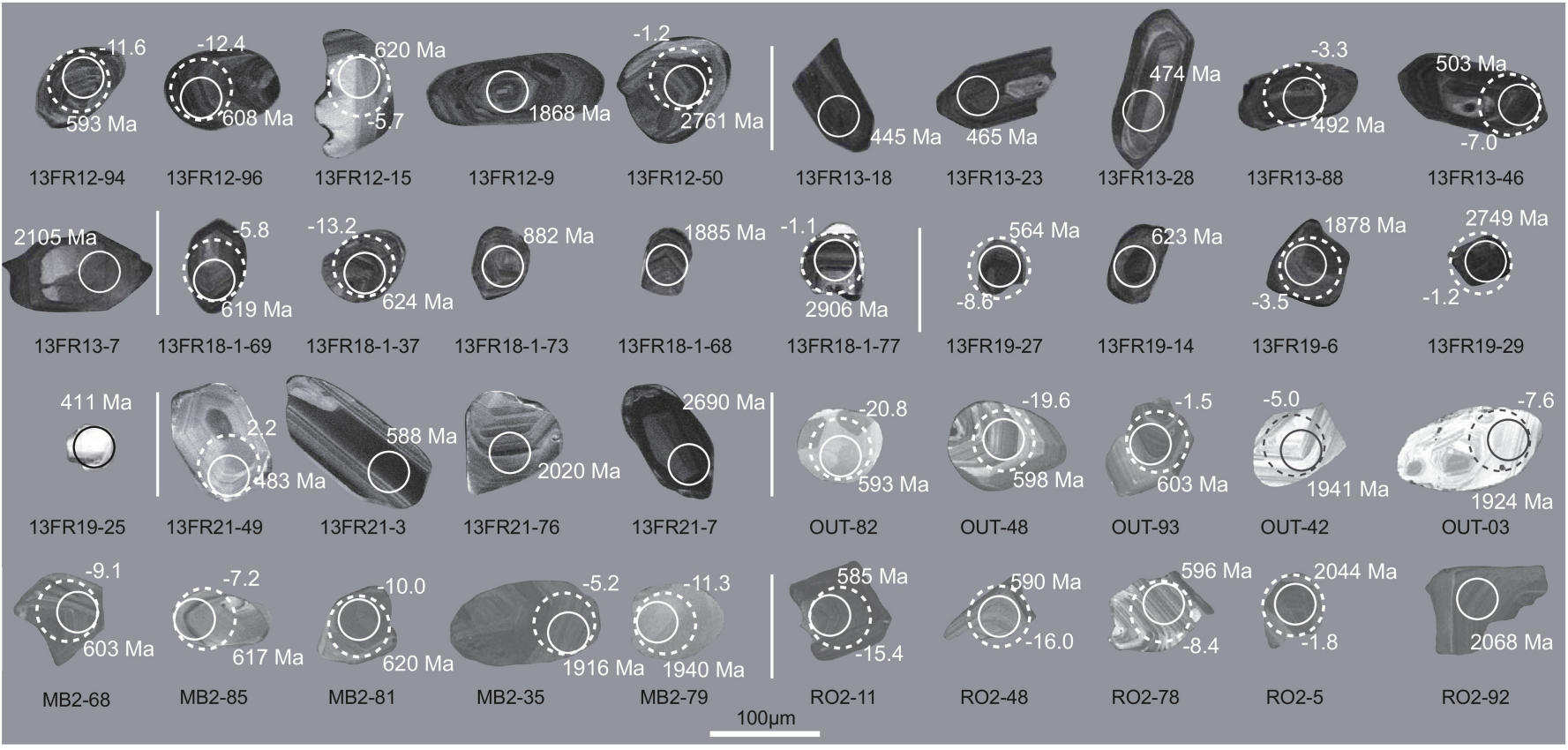

Figure 6 

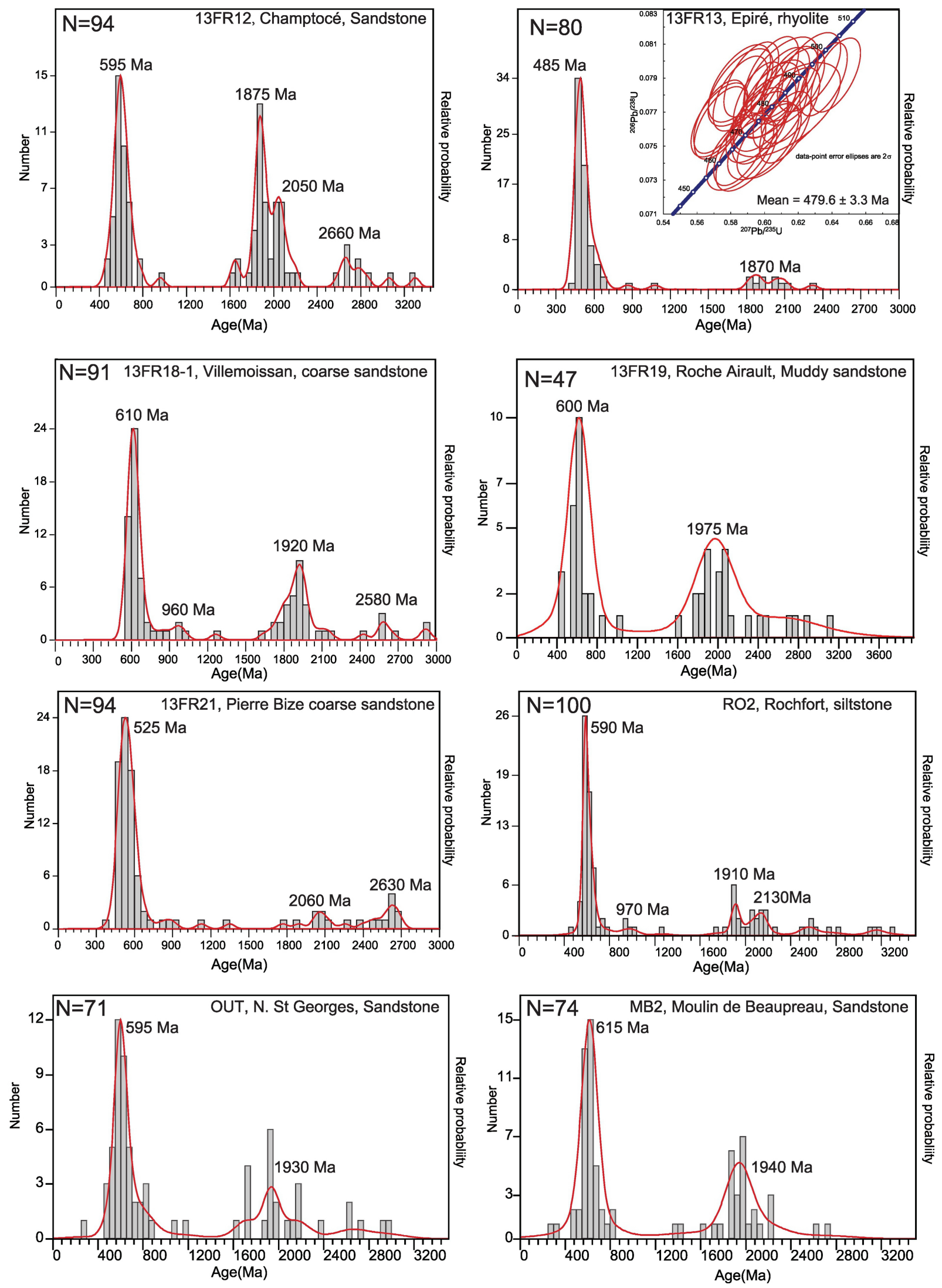

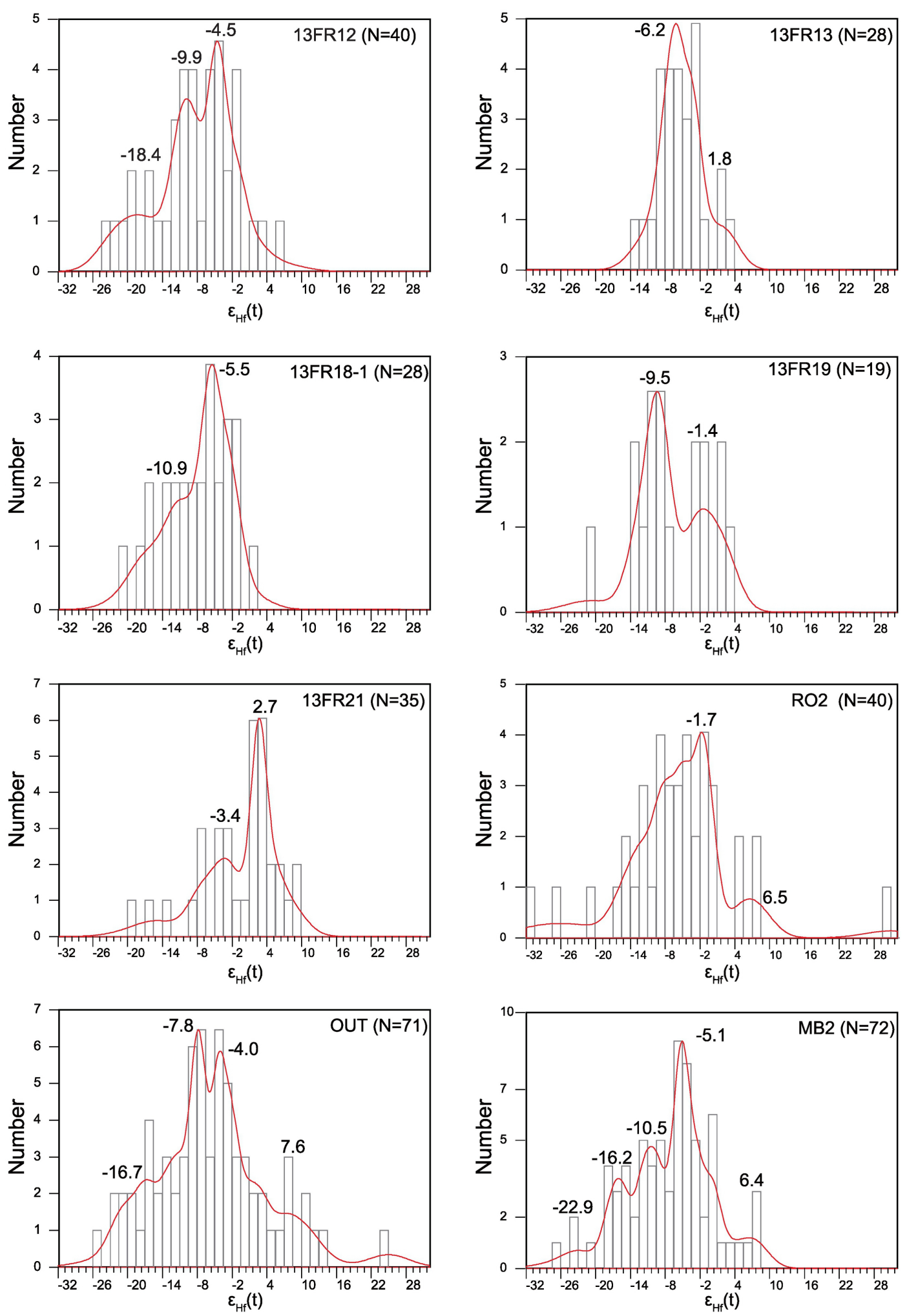

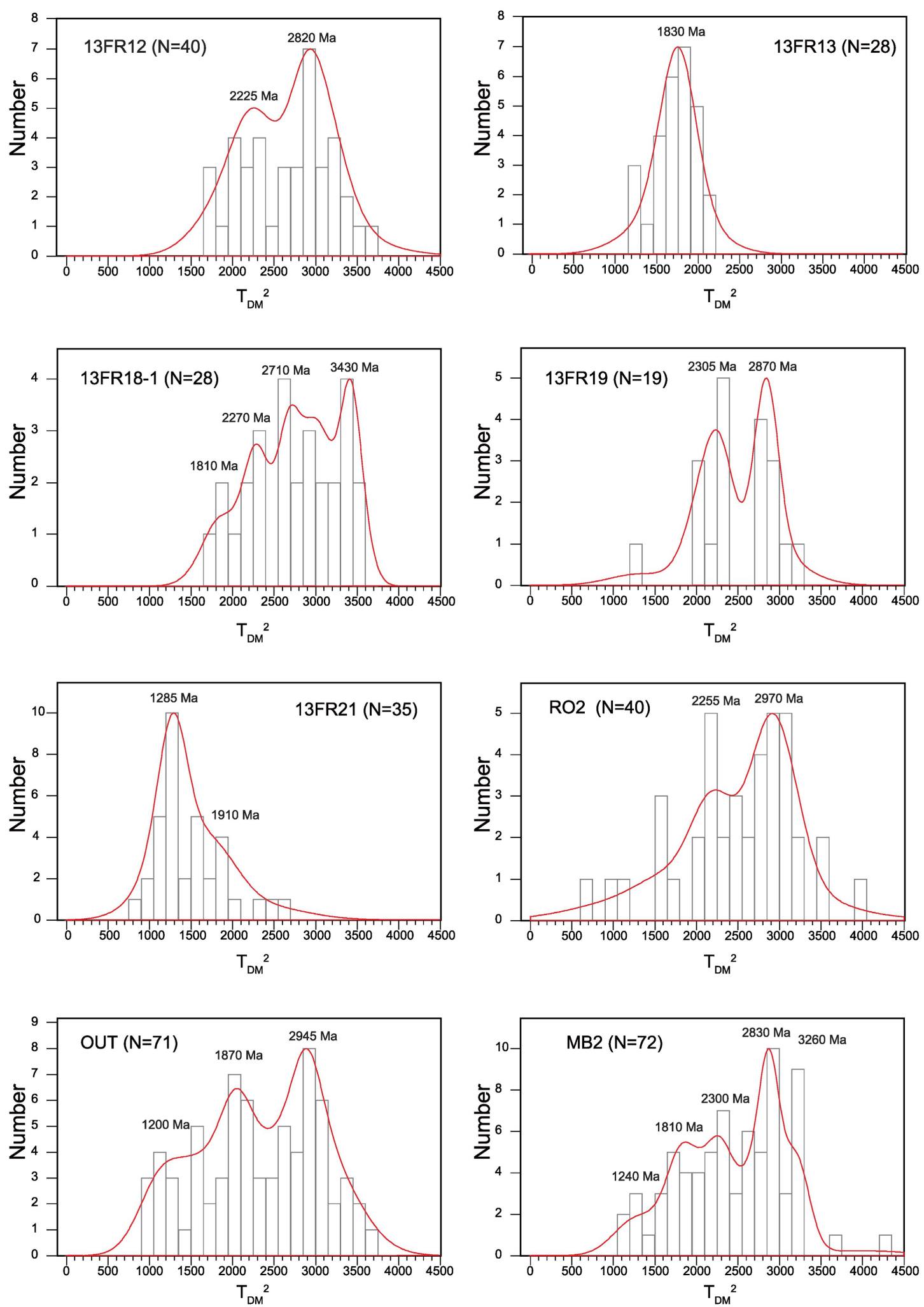

Figure 9 

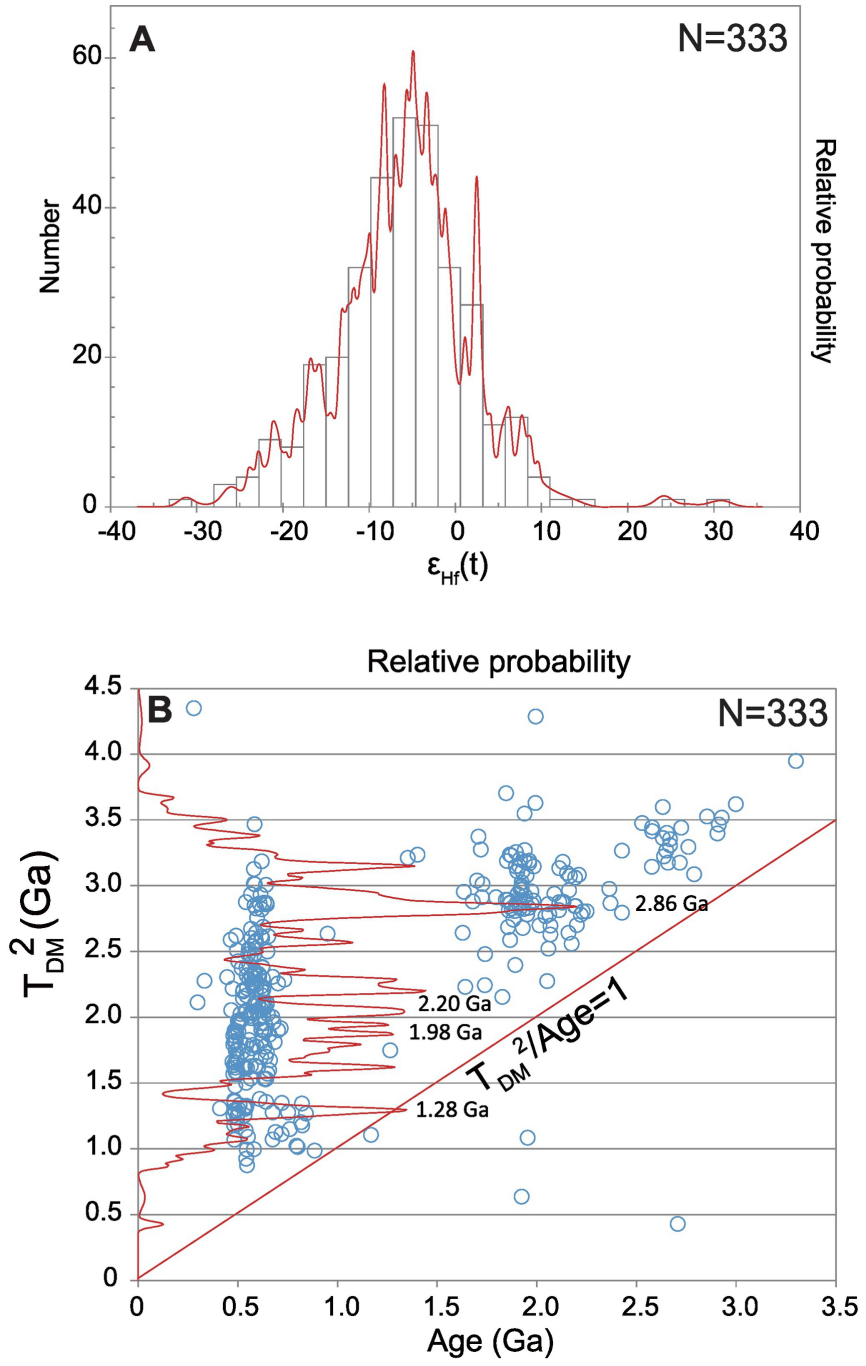

Figure 10 

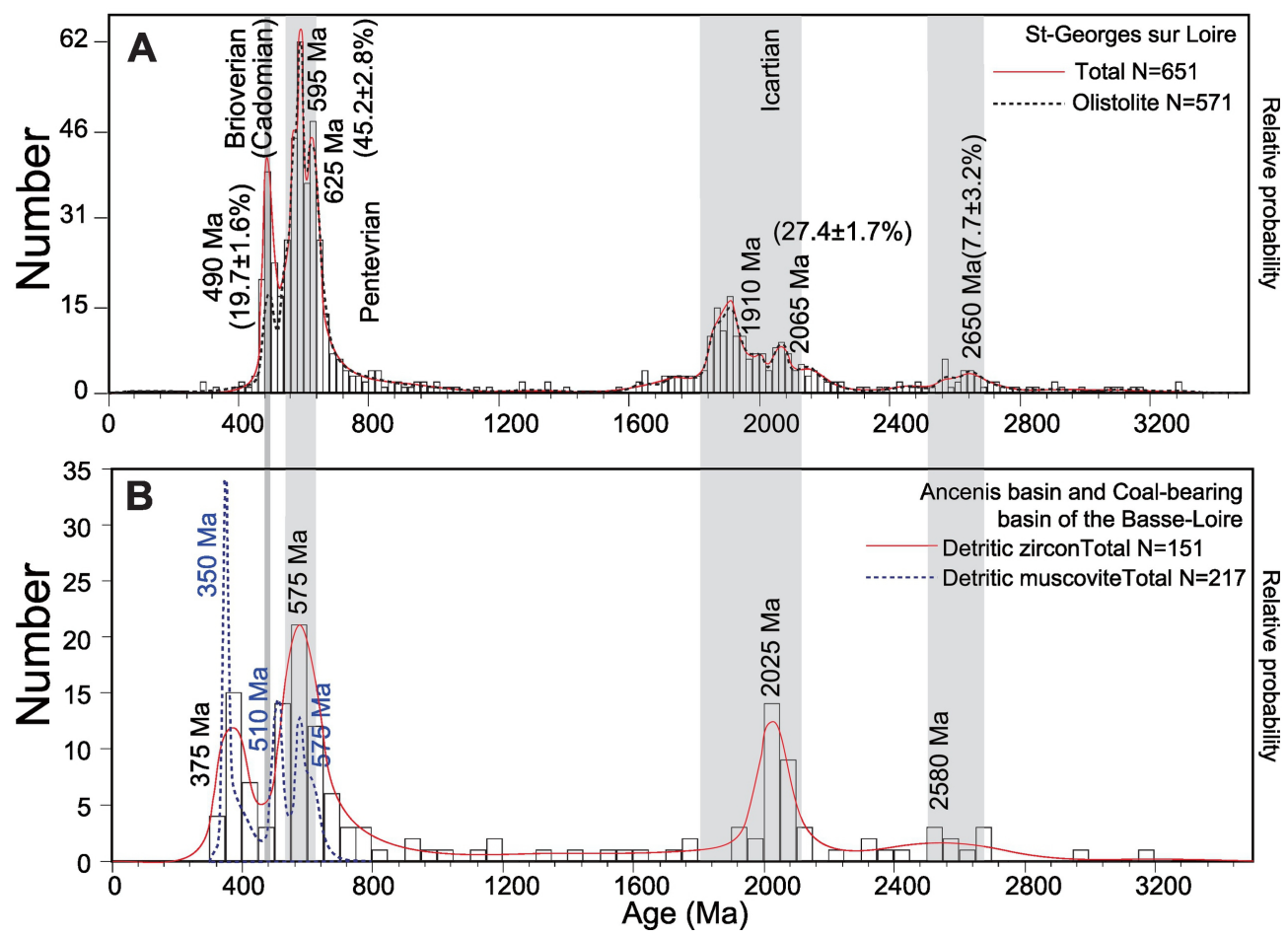

Figure 11 

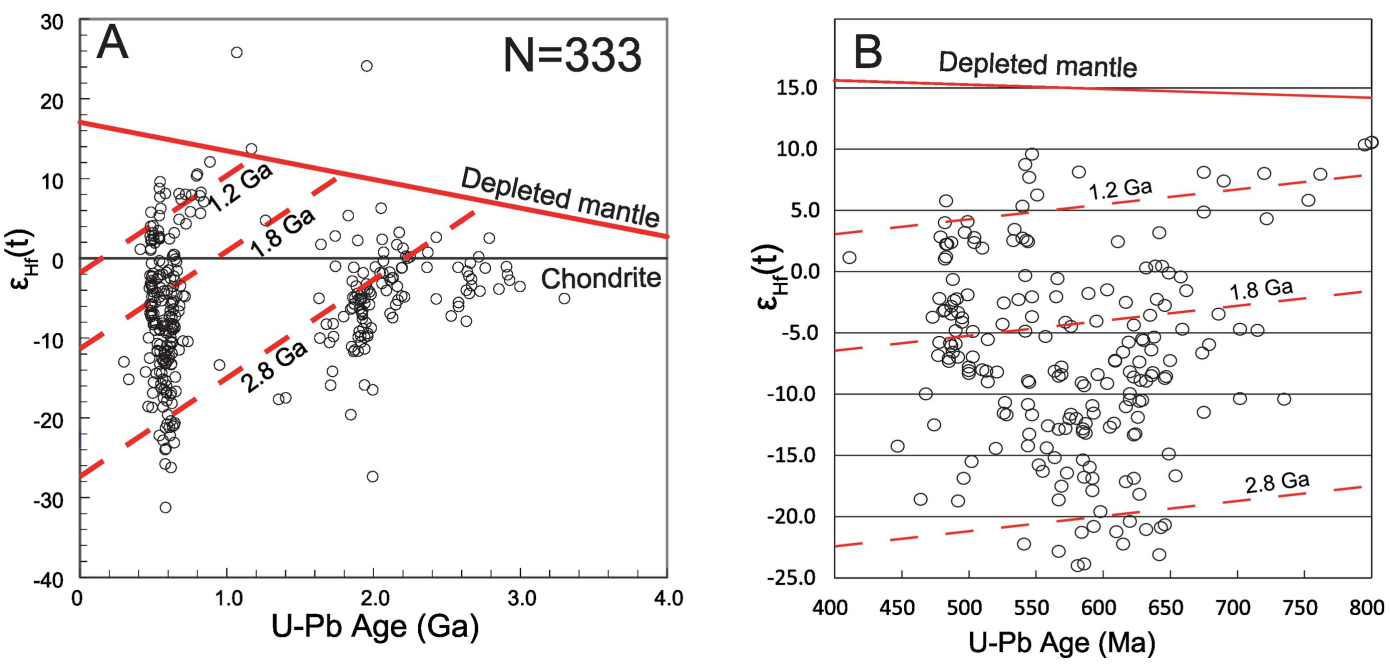

Figure 12 

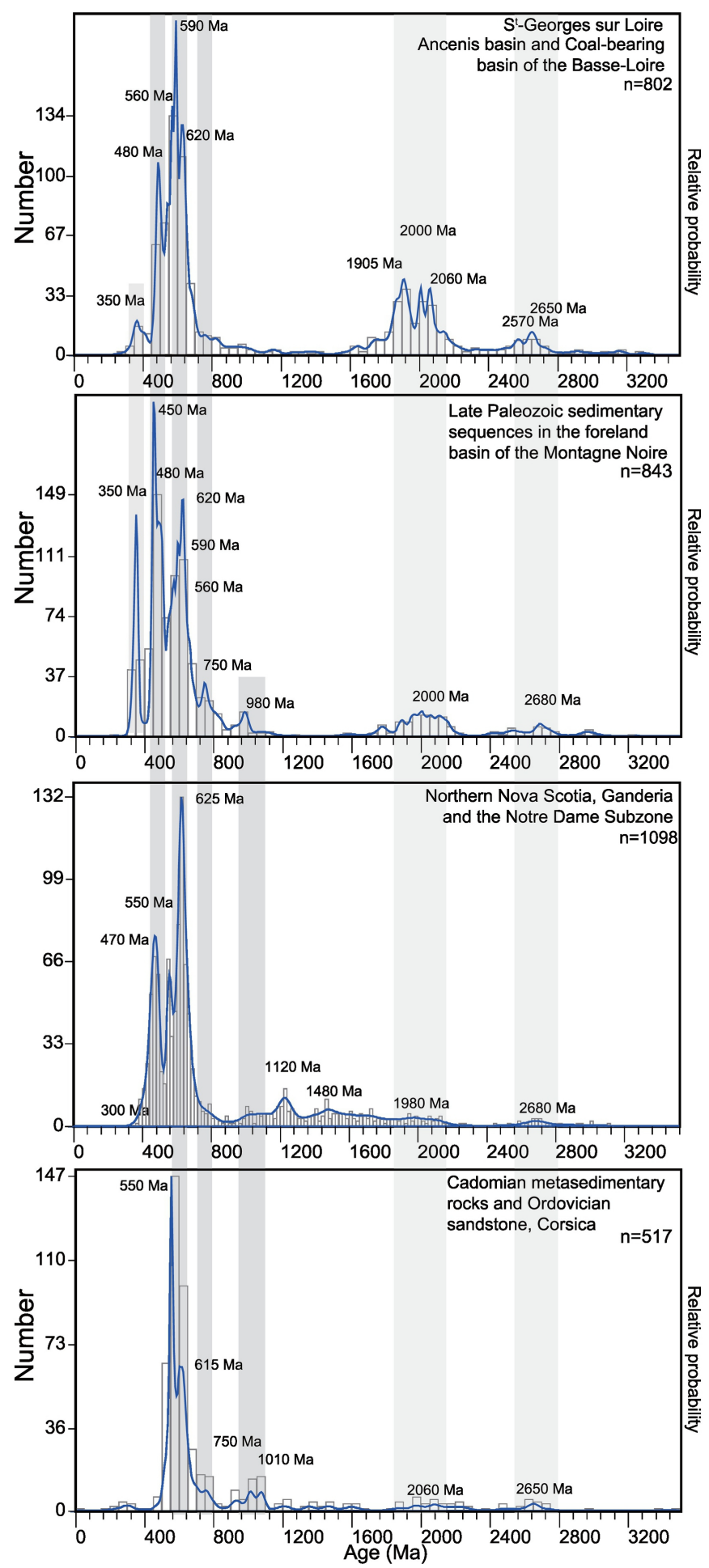

Figure 13 
Armorica

- - - - - - Cadomian belt - - ;

Trégor $\mathrm{U}$

${ }^{\mathrm{t}}$-Brieuc $\mathrm{U}_{\mathrm{S}^{\mathrm{t}} \text {-Malo }}$
Magmatic arc (missing domain)

Gondwana

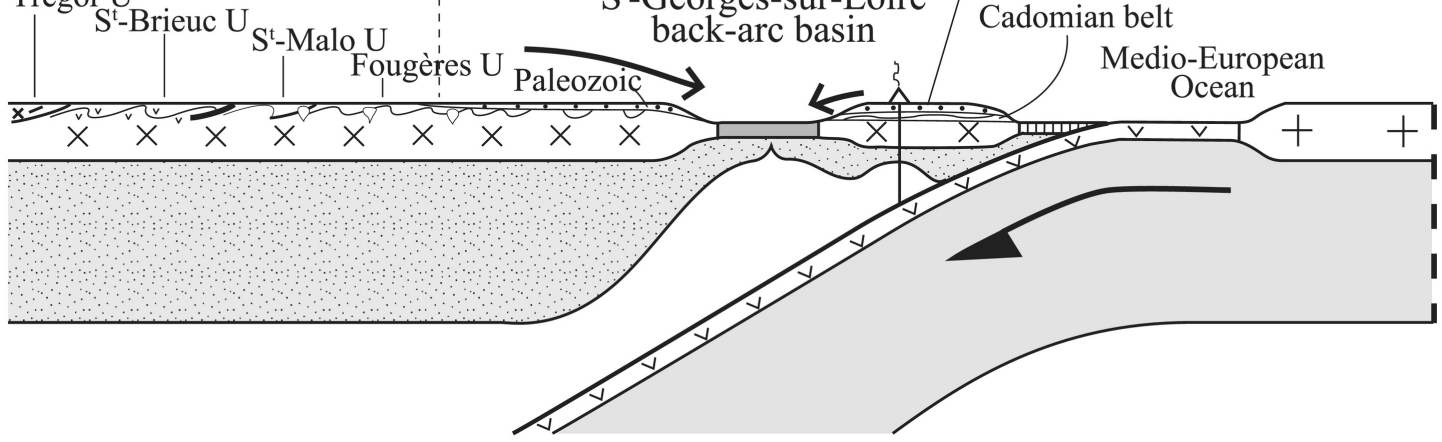

Figure 14 